Universidade de Brasília

Faculdade de Tecnologia

Departamento de Engenharia Mecânica

\title{
CONVECÇÃO NATURAL EM CAVIDADES 3D
}

\author{
Marcelo Dias de Moura
}

Orientador: Antonio Cesar Pinho Brasil Junior

Tese de Doutorado em Ciências Mecânicas

Publicação: ENM.TD-37/2016

Brasília-DF: agosto de 2016 
Universidade de Brasília

Faculdade de Tecnologia

Departamento de Engenharia Mecânica

CONVECÇÃO NATURAL EM CAVIDADES 3D

Marcelo Dias de Moura

TESE SUBMETIDA AO DEPARTAMENTO DE ENGENHARIA MECÂNICA DA FACULDADE DE TECNOLOGIA DA UNIVERSIDADE DE BRASÍLIA COMO PARTE DOS REQUISITOS NECESSÁRIOS PARA A OBTENÇÃO DO GRAU DE DOUTOR EM CIÊNCIAS MECÂNICAS.

Aprovada por:

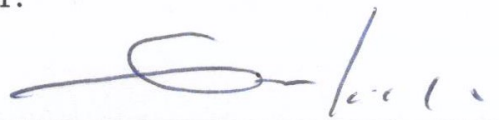

Antonio Cesar Pinho Brasil Junior, Dr., UnB

(Orientador)

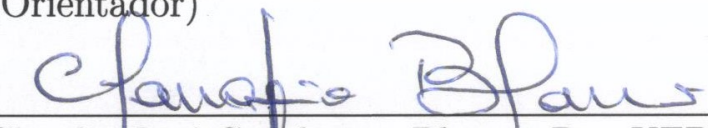

Claudio José Cavalcante Blanco, Dr., UFPA

(Examinador Externo)

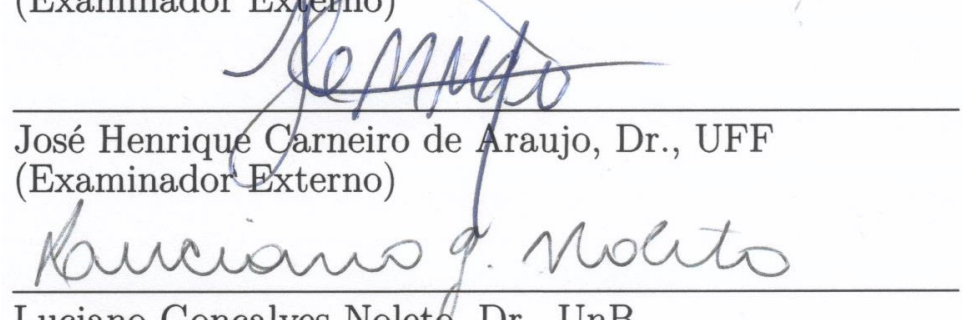

Luciano Gonçalves Noleto, Dr., UnB

(Examinador Interno)

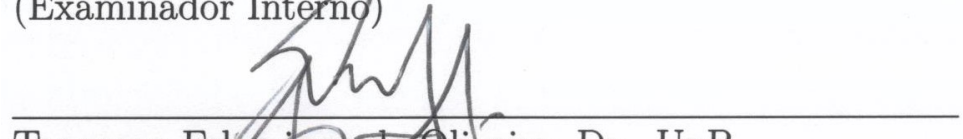

Taygoara Felerningode oliveira, Dr., UnB

(Examinadop Interno)

Carlos Alberto Gurgel Veras, Dr., UnB

(Examinador Interno Suplente)

Brasíla-DF, 09 de agosto de 2016. 
FICHA CATALOGRÁFICA

MOURA, MARCELO DIAS DE

Convecção Natural em Cavidades 3D.

[Distrito Federal] 2016.

xix, 121p., 297 mm (EnM/FT/UnB, Doutor, Ciência Mecânicas, 2016).

Tese de Doutorado - Universidade de Brasília.

Faculdade de Tecnologia.

Departamento de Engenharia Mecânica.
1. Convecção Natural
2. Cavidade
3. Simulação
4. OpenFOAM
I. $\mathrm{EnM} / \mathrm{FT} / \mathrm{UnB}$
II. Título (série)

\section{REFERÊNCIA BIBLIOGRÁFICA}

MOURA, M. D. (2016). Convecção Natural em Cavidades 3D. Tese de Doutorado em Ciências Mecânicas, Publicação ENM.TD-37/2016, Departamento de Engenharia Mecânica, Universidade de Brasília, Brasília, DF, 121p.

\section{CESSÃO DE DIREITOS}

NOME DO AUTOR: Marcelo Dias de Moura.

TÍTULO DA TESE DE DOUTORADO: Convecção Natural em Cavidades 3D.

GRAU/ANO: Doutor/2016

É concedida à Universidade de Brasília permissão para reproduzir cópias desta tese de doutorado e para emprestar ou vender tais cópias somente para propósitos acadêmicos e científicos. O autor reserva outros direitos de publicação e nenhuma parte desta tese de doutorado pode ser reproduzida sem a autorização por escrito do autor.

Marcelo Dias de Moura

Alam. Dos Lírios, 116 - Aeroporto 79.332-200 Corumbá - MS - Brasil marcelo.moura@ufms.br 
À minha mãe, Cecilia Dias Moura (in memoriam). Ao meu pai, Aureliano Moura. À minha esposa Rita Cássia de Paula Moura e meu filho Gabriel. 


\section{Agradecimentos}

Inicialmente, quero agradecer a Deus por ter me proporcionado saúde e sabedoria por ter chegado até aqui.

Agradecer à minha mãe que sempre me incentivou e que gostaria de tê-la visto assistindo a finalização desse trabalho. Ao meu pai que sempre proporcionou com muito suor os nossos estudos.

À minha esposa Rita que tem me acompanhado e ajudado desde o início quando ainda iniciava o mestrado e vivíamos somente de bolsa, morando numa quitinete, num subsolo de uma quadra comercial no Plano Piloto em Brasília.

Ao meu querido amigo e orientador prof. Brasil, pela oportunidade de desenvolver o trabalho no Laboratório de Energia e Ambiente (LEA) e por partilhar de sua experiência.

Aos colegas do LEA, Luís Aramis, Paulo Strobel, Nela, Rubéria e José Gustavo pelas várias situações que vivenciamos no laboratório.

Ao meu primo Ronald Alzamende e sua esposa Flávia pela acolhida em sua casa quando precisei ir ao Rio de Janeiro em 2013.

Ao professor Luis Fernando e Gabriel Ferreira do Grupo NUMAP-FOAM-Br-2013 da COPPE/UFRJ pelos auxílios com as ferramentas do OpenFOAM.

Aos professores Taygoara, Luciano Noleto e Armando Pires pelas contribuições e críticas ao desenvolvimento do trabalho.

Aos meu sogros, Aroldo e Fátima, e cunhados, Denise e Ronaldo pela acolhida em meu retorno à Corumbá.

Aos professores do curso de Matemática/CPAN e à UFMS por terem me liberado para cursar o doutorado.

E, por fim, agradecer à CAPES que financiou o meu afastamento através do programa PRODOUTORAL. 


\title{
Resumo
}

\section{CONVECÇÃO NATURAL EM CAVIDADES 3D}

\author{
Autor: Marcelo Dias de Moura \\ Orientador: Antonio Cesar Pinho Brasil Junior \\ Programa de Pós-graduação em Ciências Mecânicas \\ Brasília, 09 de agosto de 2016
}

A transferência de calor por convecção natural em um fluido confinado entre super-
fícies (cavidade) e a diferentes temperaturas é um problema que está relacionado com
diversas situações práticas que envolvem o condicionamento de produtos na indústria
de alimentos, a calefação ou refrigeração. O problema específico aqui analisado diz
respeito ao aquecimento transiente de uma cavidade $3 \mathrm{D}$ completamente cheia, inici-
almente a uma temperatura $T_{0}$. Em um determinado momento, todas as paredes da
caixa são aquecidos a uma temperatura $T_{W}$, maior que $T_{0}$. O calor se propaga por
convecção natural em recintos até o seu equilíbrio térmico completo. Este problema é
analisado por simulações numéricas pelo software de fluidodinâmica OpenFOAM (Open Field Operation and Manipulation) e comparado com dados experimentais: cavidade cúbica do trabalho de Lin (1982) e Lin e Akins (1983) e a cavidade paralelepípeda retangular do trabalho de Tollini (1996). Uma discussão sobre a topologia, padrões do escoamento e perda de simetria dentro da cavidade e os efeitos da convecção natural são apresentados. Verificou-se que para baixos números de Rayleigh ocorria a manutenção dos padrões nas estruturas de fluidos aquecidas (em forma de cogumelos) que se formavam na base da cavidade. Sugerindo a desestabilização dessas estruturas para Rayleigh próximos à números de transição laminar-turbulento, observados na simulação de Grandes Escalas. Foram feitas várias implementações na criação de novos Solvers para problemas de agitação/rotação, para convecção natural com Simulação de Grandes Escalas e cálculos auxiliares, que servirão como ferramenta para outros 
usuários do OpenFOAM.

Palavras-chave: Convecção Natural, Cavidade, Simulação, OpenFOAM 


\title{
Abstract
}

\section{NATURAL CONVECTION IN 3D CAVITIES}

\author{
Author: Marcelo Dias de Moura \\ Supervisor: Antonio Cesar Pinho Brasil Junior \\ Programa de Pós-graduação em Ciências Mecânicas \\ Brasília, august 9, 2016
}

The heat transfer by natural convection in a fluid confined between surfaces (cavity) and at different temperatures is a problem that is related to a number of practical situations involving conditioning products in the food industry, heating or cooling. The specific problem discussed here relates to transient heating a 3D completely filled cavity initially at a temperature $T_{0}$. At one point, all the walls of the box are heated to a temperature $T_{W}$, greater than $T_{0}$. The heat is spread by natural convection in enclosures to its full thermal equilibrium. This problem is examined by numerical simulations by software fluid dynamics OpenFOAM (Open Field Operation and Manipulation) and compared to experimental data: cubic cavity of the work Lin (1982) and Lin e Akins (1983) and the rectangular parallelepiped cavity work of Tollini (1996). A discussion of the topology, flow patterns and loss of symmetry within the cavity and the effects of natural convection are presented. It was found that for low Rayleigh numbers occurred at maintaining standards in the heated fluids structures (mushroom-shaped) which is formed at the base of the cavity. Suggesting the destabilization of these structures to Rayleigh numbers near laminar-turbulent transition, observed in simulation Large Scales. several implementations were made in the creation of new Solvers for problems of agitation/rotation for natural convection with large simulation scales and auxiliary calculations, which will serve as a tool for other users of OpenFOAM.

Keywords: Natural Convection, Cavity, Simulation, OpenFOAM 


\section{Sumário}

1 Introdução 1

1.1 Motivação . . . . . . . . . . . . . . . . . . . . . . . . . . 1

1.2 Objetivos . . . . . . . . . . . . . . . . . . . 6

1.3 Convecção Natural em Cavidades . . . . . . . . . . . . . . . . . . 7

1.4 Organização do Texto . . . . . . . . . . . . . . . . . . . . . . 8

$\begin{array}{lll}2 & \text { Formulação Matemática } & 10\end{array}$

2.1 Equações Governantes . . . . . . . . . . . . . . . . . . . . . . . . . 11

2.2 Modelagem da Turbulência . . . . . . . . . . . . . . . . . . . . . . . . . 14

$2.2 .1 \quad$ Decomposição de Reynolds . . . . . . . . . . . . . . . . . . . 15

2.2 .2 Problema de Fechamento . . . . . . . . . . . . . . . . . . . 18

$2.2 .2 .1 \quad$ Modelos a Duas Equações . . . . . . . . . . . . . . . 20

$2.2 .2 .2 \quad$ O Modelo $k-\varepsilon$. . . . . . . . . . . . . . . . . . . 24

$2.2 .3 \quad$ Simulação de Grandes Escalas - SGE . . . . . . . . . . . . . . 25

2.3 Modelo Sub-Malha de Smagorinski . . . . . . . . . . . . . . . . . . 30

2.4 Condições de Contorno . . . . . . . . . . . . . . . . . . . . . . . . . . . 31

2.4 .1 Condições de Contorno para $\phi=0$. . . . . . . . . . . . . 31

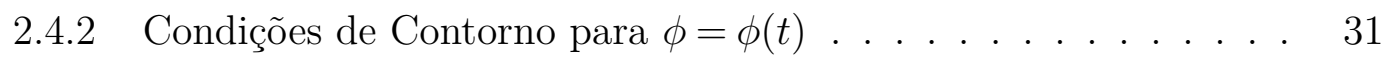

2.5 Adimensionalizações . . . . . . . . . . . . . . . . . . . . . . 33

2.5.1 Números Adimensionais Importantes para Convecção Natural 34

\begin{tabular}{lll}
\hline 3 & Metodologia Numérica & 37
\end{tabular}

3.1 Discretização pelo Método de Volumes Finitos . . . . . . . . . . . . . . 37

$3.1 .1 \quad$ Sistema Algébrico de Equações . . . . . . . . . . . . . . . . . . 44

3.1 .2 Notação da Formulação Discreta. . . . . . . . . . . . . . . . . . 45

3.2 Linguagem de Programação no OpenFOAM . . . . . . . . . . . . . . . . 47

3.3 Implementação do Código CFD no OpenFOAM . . . . . . . . . . . . . 49

3.4 Convecção Natural usando a Hipótese de Boussinesq no OpenFOAM . 52

3.4.1 Estrutura Principal para Efetuar a Simulação no OpenFOAM . 53 
3.4 .2 Pré-Processamento . . . . . . . . . . . . . . . . . . . . . 54

3.4.2.1 Construção da Geometria e da Malha. . . . . . . . . . 54

3.4 .2 .2 Configurações da Simulação . . . . . . . . . . . . 55

3.4 .3 Solução Numérica . . . . . . . . . . . . . . . . . . . . . . 57

3.4 .4 Pós-Processamento . . . . . . . . . . . . . . . . . . . . . . . 57

3.5 Implementação Numérica do Solver para o Problema de Agitação da

Cavidade. . . . . . . . . . . . . . . . . . 58

\begin{tabular}{|lll}
\hline 4 & Resultados & 63
\end{tabular}

4.1 Introdução . . . . . . . . . . . . . . . . . . . . . . . . . . . . . . 63

4.2 Comparação com Dados Experimentais . . . . . . . . . . . . 67

4.3 Caracterização da Convecção Natural na Cavidade . . . . . . . . . . . 75

4.3 .1 Relação entre Energia Cinética e o Surgimento dos "Cogumelos"] 83

4.3 .2 Distribuição de Nusselt nas Paredes . . . . . . . . . . . . . . . 85

4.3 .3 Simetria $\ldots \ldots \ldots \ldots$. . . . . . . . . . . . . . . . . . 87

4.4 Efeitos da Agitação no Processo de Convecção Natural . . . . . . . . . 94

4.5 Turbulência . . . . . . . . . . . . . . . . . . . . . . . . . . . . . . 99

$\begin{array}{lll}5 \text { Conclusões } & 104\end{array}$

\begin{tabular}{ll}
\hline REFERÊNCIAS BIBLIOGRÁFICAS & 107
\end{tabular}

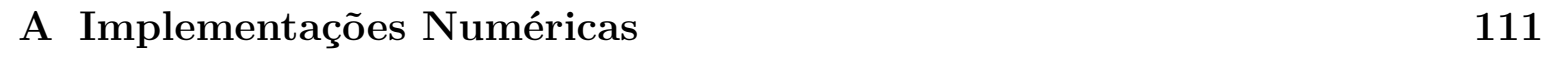

A.1 Implementação Numérica do Solver para o Problema de Agitação da Cavidade . . . . . . . . . . . . . . . . . . . . 111 


\section{Lista de Figuras}

1.1 Correlações de Nusselt vs Rayleigh para a Cavidade. . . . . . . . . . . 2

1.2 Categorias de Aquecimento da cavidade: (a) cavidades aquecidas pelas paredes laterais; (b) cavidades aquecidas pela parede inferior e (c) Todas as paredes aquecidas. . . . . . . . . . . . . . 2

1.3 Características da Convecção Natural na Cavidade: Escoamento. . . . . 3

1.4 Estruturas Intermitentes de Fluido Aquecido numa Superfície Horizontal (Adaptado de (SPARROW; HUSAR; GOLDSTEIN] 1970)) . . . . . . . 4

1.5 Características da Convecção Natural na Cavidade: Temperatura. . . . 6

2.1 Cavidade paralelepípeda retangular . . . . . . . . . . . . . . . . 10

2.2 Evolução temporal da velocidade calculada por DNS, LES e RANS em um escoamento turbulento (Adaptado de (STOLL, 2014)). . . . . . . . 15

2.3 Espectro de Energia do Escoamento Turbulento (Adaptado de ((VILLI-

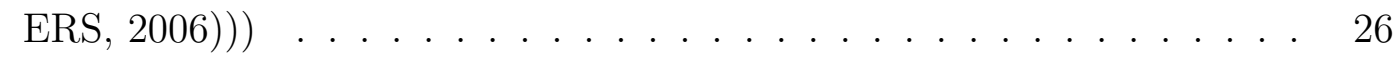

2.4 Resíduo Aplicado à Variável $\phi$ (Adaptado de (POPE $[2000)$ ) . . . . . . 28

2.5 Representação da Imposição da Oscilação no Centro do Cubo. . . . . . . 32

3.1 Discretização do Domínio da Solução (Adaptado de (OPENCFD, $2014 \mathrm{a})$ ) 38

3.2 Parâmetros na Discretização do Método de Volumes Finitos (Adaptado

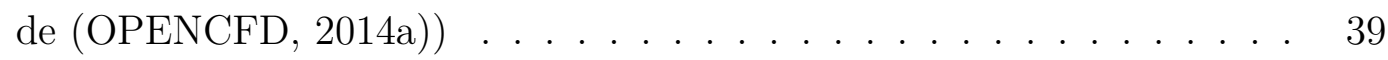

$3.3 \quad$ Bibliotecas do OpenFOAM (Adaptado de (SILVA] 2008)) . . . . . . . . 49

3.4 Operações da Classe geometricField<Type > usando Operações Implícitas (fvm) e Explícitas (fvc) (Adaptado de (OPENCFD, 2014a)). . 51

3.5 (a) Estrutura de Diretório de Caso (Adaptado de (OPENCFD, 2014b)); (b) Arquivos do Solver do OpenFOAM. . . . . . . . . . . . . . . . . 52

$3.6 \quad$ Estrutura de Arquivos do solver buoyantBoussinesqPimpleFoam 54

3.7 Conteúdo do solver buoyantBoussinesqPimpleFoam . . . . . . . 58

3.8 Representação Simplificada da Execução do solver buoyant Bou s s ine sqP impleF oam

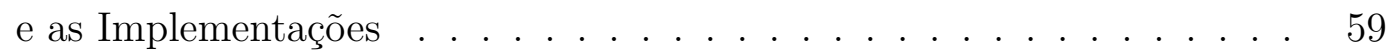


3.9 Representação dos solvers, Alteração no Método de LES e Inclusão de Aplicações para o Cálculo de Nu e K. . . . . . . . . . . . . . . . . . 62

4.1 Geometrias Utilizadas nos Estudos de Casos para a Convecção Natural: (a) Paralelepípedo Retangular; (b) Cubo. . . . . . . . . . . . . . . . . . 64

4.2 Posicionamentos dos Locais de Extração nas Linhas sobre os Planos: (a) $y=H / 2(\operatorname{para} z=29 H / 30, z=3 H / 4, z=H / 2, z=H / 4$ e $z=H / 30) ;(\mathrm{b})$ $x=H / 2($ para $z=29 H / 30, z=3 H / 4, z=H / 2, z=H / 4$ e $z=H / 30) ;$ (c) $y=H / 2($ para $x=29 H / 30, x=3 H / 4, x=H / 2, x=H / 4$ e $x=H / 30) .65$

4.3 Planos de Visualização dos Resultados para Contornos e Vetores: (a)

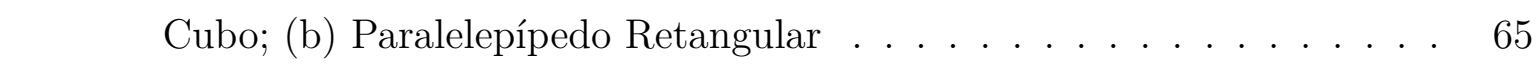

4.4 Detalhe da Malha Utilizada no Teste de Validação. . . . . . . . . . . . 67

4.5 Teste de Malha para Valores de (a) Temperatura e (b) Nusselt variando

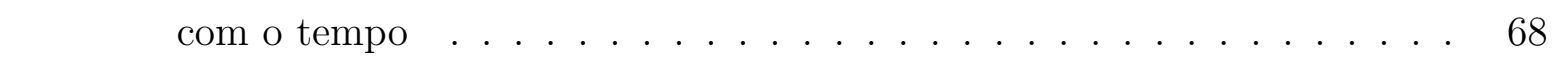

$4.6 \quad$ Teste de Malha em $t *=76$ para Valores de (a) Temperatura e (b) Velocidade de camada limite na direção $z$ da linha em $(x, H / 2, H / 2)$. . . . 69

4.7 Comparação de Nusselt $\times$ Rayleigh do Cubo com dados experimentais

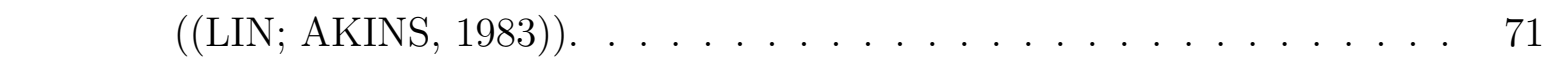

4.8 Comparação de Nusselt $\times$ Rayleigh do Cubo com dados experimentais ((TOLLINI, 1996)). . . . . . . . . . . . . . . . 72

4.9 Comparação da Velocidade $U_{z}$ com dados experimentais para o Cubo

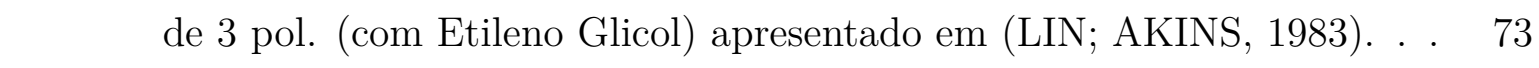

4.10 Perfis de Velocidade sobre o plano $y=H / 2$ na direção $x$ com a variação da altura (z) para o Cubo de 3 pol. (com Etileno Glicol) apresentado

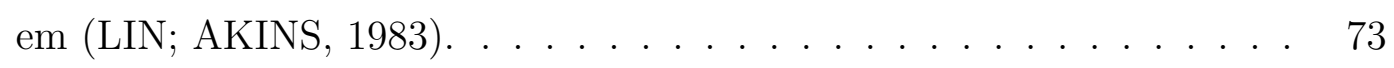

4.11 Perfis de Velocidade sobre o plano $y=H / 2$ na direção $x$ com a variação \begin{tabular}{|c|}
\hline da altura $(z)$ para o Cubo de 3 pol. (com Etileno Glicol) apresentado \\
\hline em $($ C
\end{tabular} em (LIN; AKINS, 1983). . . . . . . . . . . . . . . . . . . 74

4.12 Perfis de Velocidade sobre o plano $y=H / 2$ na direção $x$ com a variação \begin{tabular}{|c|}
\hline da altura $(z)$ para o Cubo de 3 pol. (com Etileno Glicol) apresentado \\
\hline
\end{tabular} em (LIN; AKINS, 1983). . . . . . . . . . . . . . . . 74

4.13 Características da Convecção Natural na Cavidade: Escoamento. . . . . 75 4.14 Visualização das Características do Cubo em $x=H / 2$ para Ra $=4 \times 10^{6} 79$ 4.15 Visualização das Características do Cubo em $y=H / 10$ para Ra $=4 \times 10^{6} 80$ 4.16 Visualização das Características do Paralelepípedo Retangular em $x=$ \begin{aligned} \hline$/ 2$ para $\mathrm{Ra}=4 \times 10^{6} \ldots \ldots \ldots \ldots \ldots \ldots \ldots & . \ldots \ldots \ldots\end{aligned}$

4.17 Visualização das Características do Paralelepípedo Retangular em $y=$ $H / 10$ para $\mathrm{Ra}=4 \times 10^{6} \ldots \ldots \ldots \ldots 2$ 
4.18 Características da Convecção Natural na Cavidade. . . . . . . . . . . . 82

4.19 Variação da Energia Cinética no Cubo para Diferentes $T_{W}$. . . . . . . . 83

4.20 Variação da Energia Cinética no Paralelepípedo para Diferentes $T_{W}$. . . 84

4.21 Formação de Padrões Relacionados aos Picos de Energia Cinética no Cubo. . . . . . . . . . . . . . . . . . . . 84

4.22 Formação de Padrões Relacionados aos Picos de Energia Cinética no Paralelepípedo. . . . . . . . . . . . . . . . 85

4.23 Visualização da Variação de Nusselt nas Faces do Cubo. . . . . . . . . 86

4.24 Variação de Nusselt nas Faces do Cubo. . . . . . . . . . . . . . . . . . . 86

4.25 Visualização da simetria no cubo para $\mathrm{Ra}=2 \times 10^{5}$. . . . . . . . . . . 87

4.26 Visualização da simetria no cubo para $\mathrm{Ra}=2 \times 10^{6}$. . . . . . . . . . . 88

4.27 Visualização da simetria no cubo para $\mathrm{Ra}=4 \times 10^{6}$. . . . . . . . . . . 89

4.28 Visualização da simetria no Paralelepípedo para $\mathrm{Ra}=1,58 \times 10^{7}$. . . . 90

4.29 Visualização da simetria no cubo para $\mathrm{Ra}=7,04 \times 10^{6}$. . . . . . . . . 91

4.30 Visualização da simetria no cubo para $\mathrm{Ra}=2 \times 10^{7}$. . . . . . . . . . . 92

4.31 Visualização da simetria no cubo para $\mathrm{Ra}=8 \times 10^{7}$. . . . . . . . . . . 93

4.32 Visualização da simetria no cubo para $\mathrm{Ra}=7 \times 10^{8}$. . . . . . . . . . . 94

4.33 Agitação do Fluido devido a $\phi(t)$ - Isosuperfícies de $\theta$. . . . . . . . . . 95

4.34 Influência da Oscilação na Convecção Natural - Temperatura. .... 97

4.35 Influência da Oscilação na Convecção Natural - Nusselt. . . . . . . . . . . 98

4.36 Influência da Oscilação na Convecção Natural - Temperatura. .... . 99

4.37 Critério para Transição à Turbulência para Placa Plana Vertical (Adptado de (BEJAN, 2004)). . . . . . . . . . . . . . . . . . 100

4.38 Comparação de Nusselt $\times$ Rayleigh do Cubo com dados experimentais

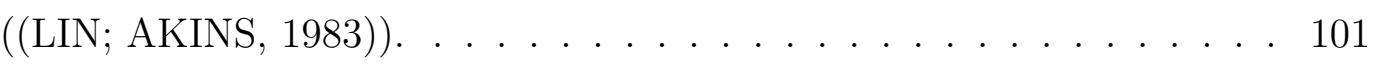

4.39 Formação de Padrões Relacionados aos Picos de Energia Cinética no Paralelepípedo para o Caso Turbulento. . . . . . . . . . . . . . . . . . . 101

4.40 Visualização das Características do Paralelepípedo Retangular em $x=$ $H / 2$ para $\mathrm{Ra}=6,77 \times 10^{9}$. . . . . . . . . . . . . . . . . . . 102

4.41 Visualização das Características do Paralelepípedo Retangular em $x=$ $H / 2$ para $\mathrm{Ra}=6,77 \times 10^{9}$. . . . . . . . . . . . . . . . 103 


\section{Lista de Tabelas}

3.1 Notação da Discretização por Volumes Finitos . . . . . . . . . . . . . . 46

4.1 Parâmetros Utilizados nas Simulações de Validação da Malha e em Comparação com Dados Experimentais. . . . . . . . . . . . . . . . . . 66

4.2 Tempo de Execução . . . . . . . . . . . . . . . . . . . . . . . . . 67

4.3 Parâmetros Utilizados nas Simulações para Análise e Caracterização do Problema. . . . . . . . . . . . . . . . . . 76

4.4 Parâmetros Utilizados na Simulação da Agitação da Cavidade . . . . . 96 


\section{Lista de Códigos}

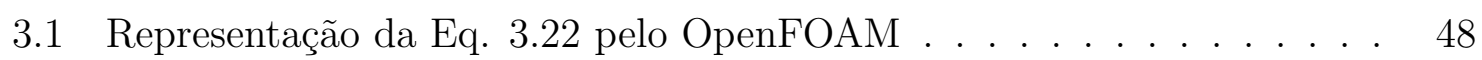

A.1 Arquivo principal g_buoyantBoussinesqPimpleFoam.C ..... 111

A.2 Arquivo de Criação de Campos createFields.H . . . . . . . . . . . 112

A.3 Arquivo calcgandomega.H . . . . . . . . . . . . . . 117

A.4 Arquivo UEqn.H . . . . . . . . . . . . . . . . . . . . . . . 118

A.5 Arquivo TEqn.

A.6 Arquivo pEqn.H . . . . . . . . . . . . . . . . . . . . . . . . . . . . 119 


\section{Lista de Símbolos, Nomenclatura e Abreviações}

\section{Letras Latinas}

L Tensor de Leonard

R Tensor de Reynolds sub-malha

C Tensão cruzada

I Tensor Identidade

U Velocidade Média

u' Flutuação de Velocidade

$\mathcal{P}_{k} \quad$ Produção de $k$

$\mathrm{Ra}_{\omega} \quad$ Número de Rayleygh rotacional, $\frac{\Omega \beta\left(T_{w}-T_{0}\right) H^{4}}{\nu \alpha}$

$\mathrm{Ra}^{*} \quad$ Número de Rayleygh local, $\left[\frac{g \beta\left(T_{w}-\bar{T}\right) H^{3}}{\nu \alpha}\right]$

Re Número de Reynolds, $\sqrt{\frac{\mathrm{Gr}}{\mathrm{Pr}}}$

D Tensor taxa de deformação

g Vetor gravidade

S Parte simétrica do tensor taxa de deformação

$C_{P} \quad$ Calor específico

F $\quad$ Função de Combinação do Modelo SST

$g \quad$ Aceleração da gravidade

H Altura da cavidade 
$h \quad$ Coeficiente de transferência de calor por convecção

$K \quad$ Energia cinética adimensional, $K^{\prime} /\left[(1 / 2) \rho \operatorname{Ra}(\alpha / H)^{2}\right]$

$k \quad$ Condutividade térmica

$k \quad$ Energia Cinética de Turbulência

L Largura da cavidade

$N u \quad$ Número de Nusselt, $h H / k$

$p \quad$ Pressão adimensional, $p^{\prime} /\left(\rho u^{* 2}\right)$

$\operatorname{Pr} \quad$ Número de Prandtl, $\nu / \alpha$

$q^{\prime \prime \prime} \quad$ Termo de fonte na equação de conservação de energia (Eq. 2.3)

Ra Número de Rayleygh, $\left[g \beta\left(T_{w}-T_{0}\right) H^{3}\right] /[\nu \alpha]$

T Temperatura

$t \quad$ Tempo adimensional, $t^{\prime} / t^{*}$

$t^{*} \quad$ Escala de tempo, $\left(H^{2} / \alpha\right) \mathrm{Ra}^{-0.5}$

$T_{0} \quad$ Temperatura inicial

$T_{w} \quad$ Temperatura final

$u^{*} \quad$ Escala de velocidade, $(\alpha / H) \mathrm{Ra}^{0.5}$

$U_{0} \quad$ Velocidade de referência, $\left[\frac{\alpha}{H} \sqrt{\mathrm{Ra}}\right]$

$W \quad$ Espessura da cavidade

$\mathbf{u} \quad$ Velocidade adimensional, $\left(\mathbf{u}^{\prime} / u^{*}\right)$

$\mathbf{x} \quad$ Vetor posição adimensional, $\mathbf{x}^{\prime} / H$

$\mathbf{F}_{\text {centrífuga }}$ Força centrífuga

$\mathbf{F}_{\text {coriolis }}$ Força de Coriolis

$\mathbf{F}_{\text {Euler }}$ Força de Euler

\section{Letras Gregas}


$\alpha \quad$ Difusividade térmica

$\beta \quad$ Coeficiente de expansão volumétrica

$\boldsymbol{\tau} \quad$ Tensor de Reynolds

$\tau^{S G} \quad$ Tensor sub-malha

$\Delta t \quad$ Passo de tempo adimensional

$\Delta \quad$ Variação, Intervalo

$\Gamma \quad$ Contornos da cavidade (paredes)

$\mu \quad$ Viscosidade Dinâmica

$\nu \quad$ Viscosidade Cinemática

$\nu_{t} \quad$ Viscosidade Turbulenta

$\nu_{S G S}$ Viscosidade sub-malha

$\Omega \quad$ Domínio computacional da cavidade (volume)

$\omega \quad$ Freqüência de Turbulência

$\phi \quad$ Variável utilizada no processo de filtragem em LES

$\rho \quad$ Massa específica

$\theta \quad$ Temperatura adimensional, $\left(T-T_{0}\right) /\left(T_{w}-T_{0}\right)$

$\varepsilon \quad$ Taxa da Dissipação de $k$

$\varphi \quad$ Variável de Integração Temporal

$L_{\varepsilon} \quad$ Escala de Dissipação

\section{Sobrescrito}

' Variável com dimensões

* Escala característica

\section{Subscrito}

$0 \quad$ Valores iniciais em $t=0$ 
$w \quad$ Parede

Siglas

DNS Direct Numerical Simulation

LES Large Eddy Simulation

MVF Método de Volumes Finitos

OpenFOAM Open Field Operation and Manipulation

ParaView Open-source, multi-platform data analysis and visualization application RANS Reynolds Averaged Navier-Stokes

Salomé The Open Source Integration Platform for Numerical Simulation SGS Sub Grid Stress 


\section{Capítulo 1}

\section{Introdução}

\subsection{Motivação}

A transferência de calor por convecção natural em um fluido confinado entre superfícies (cavidade) a diferentes temperaturas é um problema que está relacionado com diversas situações práticas: processos de esterilização de produtos líquidos em embalagens na indústria de alimentos, coletores solares, resfriamento de componentes eletrônicos, aquecimento ou arrefecimento em edifícios e a transferência de calor dentro dos tanques de armazenamento térmico, são exemplos de aplicação para este tipo de problema convectivo.

O fenômeno da convecção natural em uma cavidade é tão heterogêneo quanto a geometria, a orientação da cavidade, assim como das correlações entre o número de Nussel e Rayleigh (figura (1.1)). A julgar pelo número de aplicações de engenharia em potencial, os fenômenos que ocorrem na cavidade podem ser organizados, a grosso modo, em duas grandes categorias: (1) cavidades aquecidas pelas paredes laterais, e (2) cavidades aquecidas pela parede inferior (figura (1.2)). 


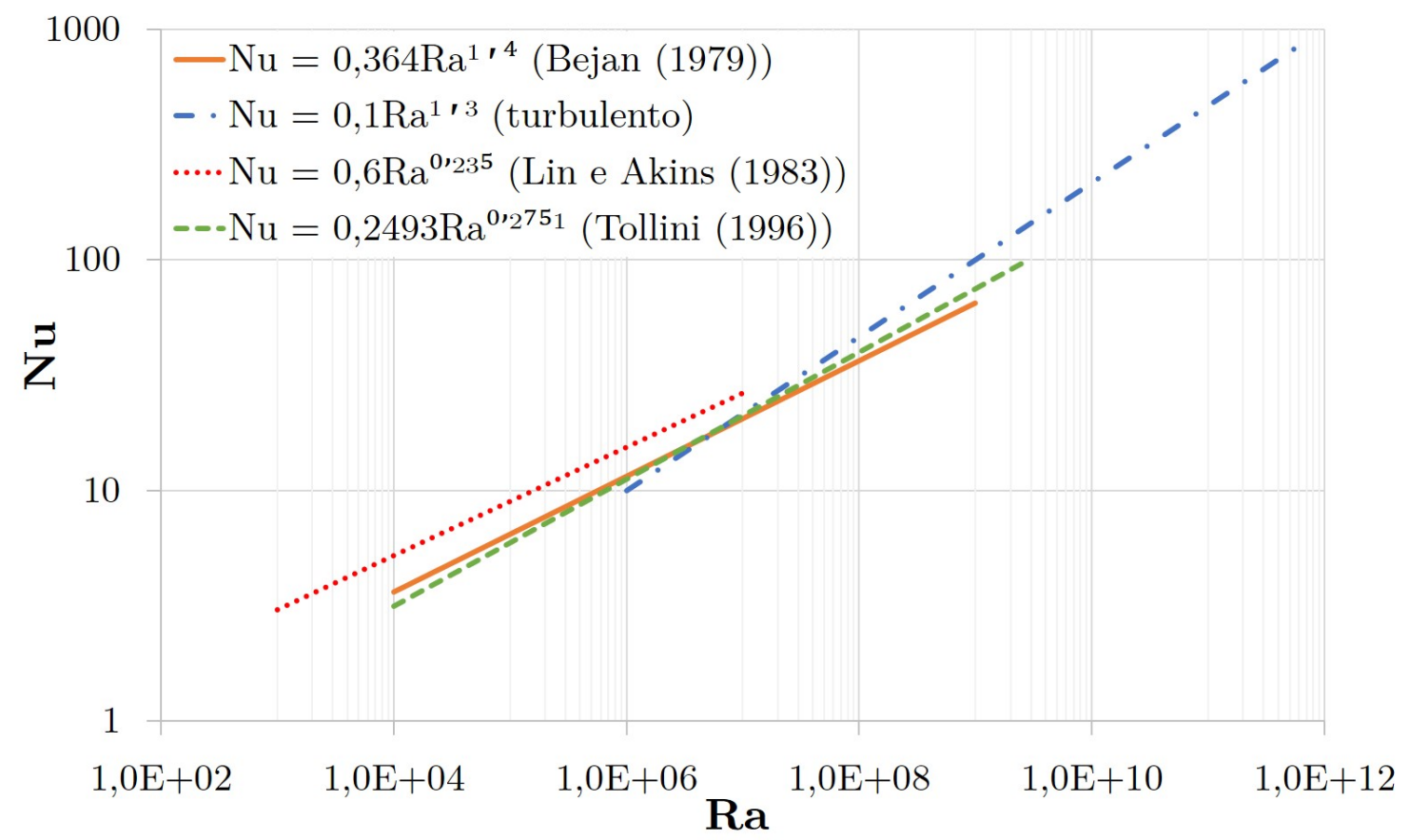

Figura 1.1: Correlações de Nusselt vs Rayleigh para a Cavidade.

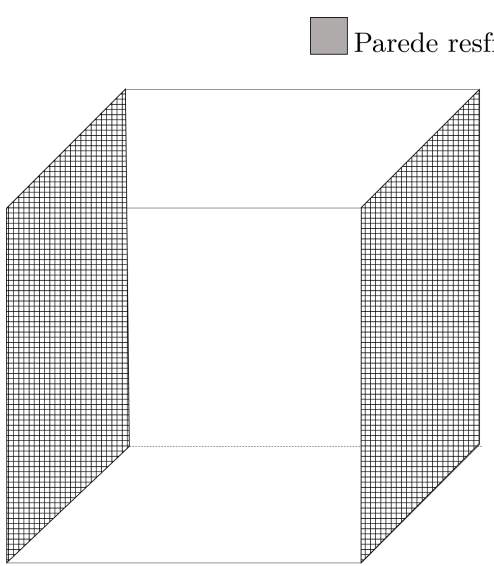

(a)

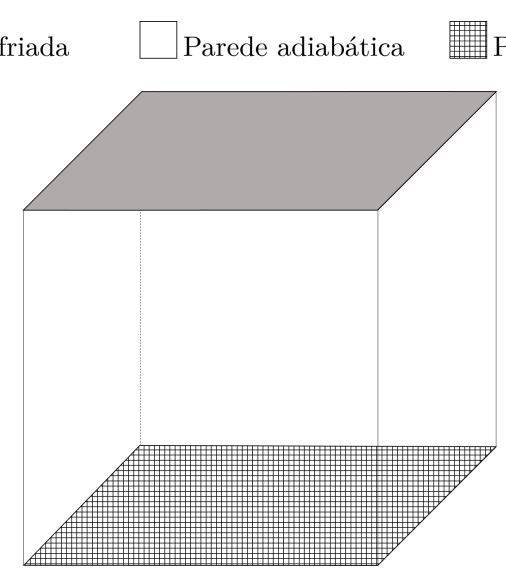

(b)

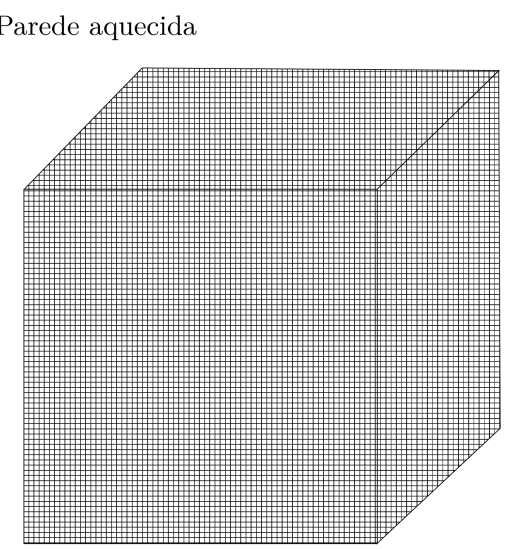

(c)

Figura 1.2: Categorias de Aquecimento da cavidade: (a) cavidades aquecidas pelas paredes laterais; (b) cavidades aquecidas pela parede inferior e (c) Todas as paredes aquecidas.

$\mathrm{Na}$ maioria dos estudos de aquecimento por convecção natural transiente em cavidades encontrados na literatura, é observado um comportamento interessante do campo de escoamento e temperatura (conforme ilustrado na figura (1.3)):

- Na fase inicial em que as paredes são aquecidas, camadas limites são formadas 
nas paredes laterais verticais (Região I) - devido à força de empuxo, pela presença do gradiente de massa específica (ocasionado pelo gradiente de temperatura) e forças de campo (gravitacional, centrífuga ou Coriolis);

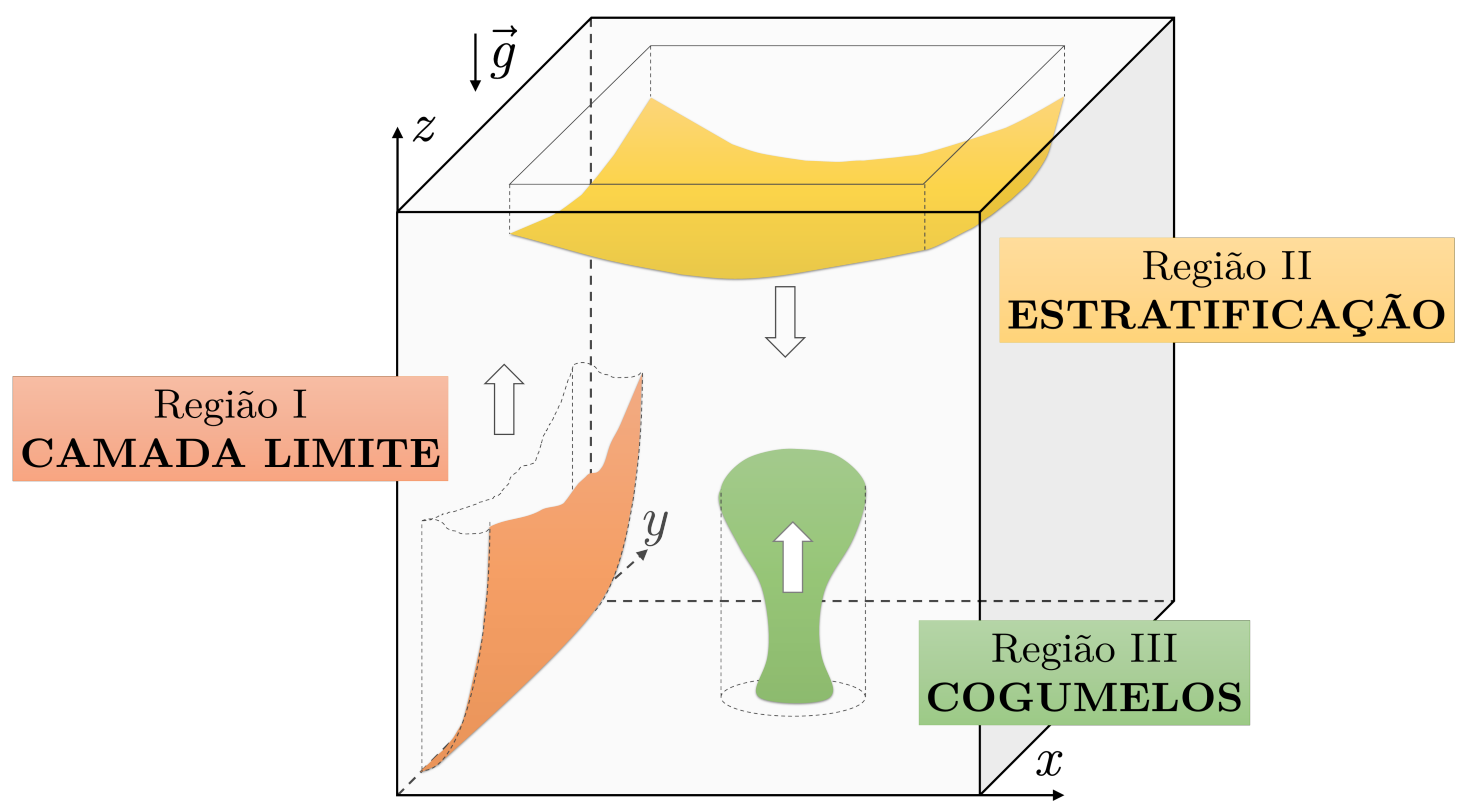

Figura 1.3: Características da Convecção Natural na Cavidade: Escoamento.

- Recirculações internas e um campo oscilatório térmico é observado - a força gravitacional age no fluido mais denso e pesado, fazendo-o descer e, interage com as camadas de fluido mais leves que sobem, formando células de circulação;

- Depois disso, a zona central é estabelecida e uma estratificação térmica é observada (Região II).

- Uma terceira situação (Região III) é o surgimento de estruturas intermitentes em formas de cogumelos - conhecidas como térmicas, essas estruturas se formam em superfícies horizontais aquecidas e voltadas para cima (ver figura 1.4). 


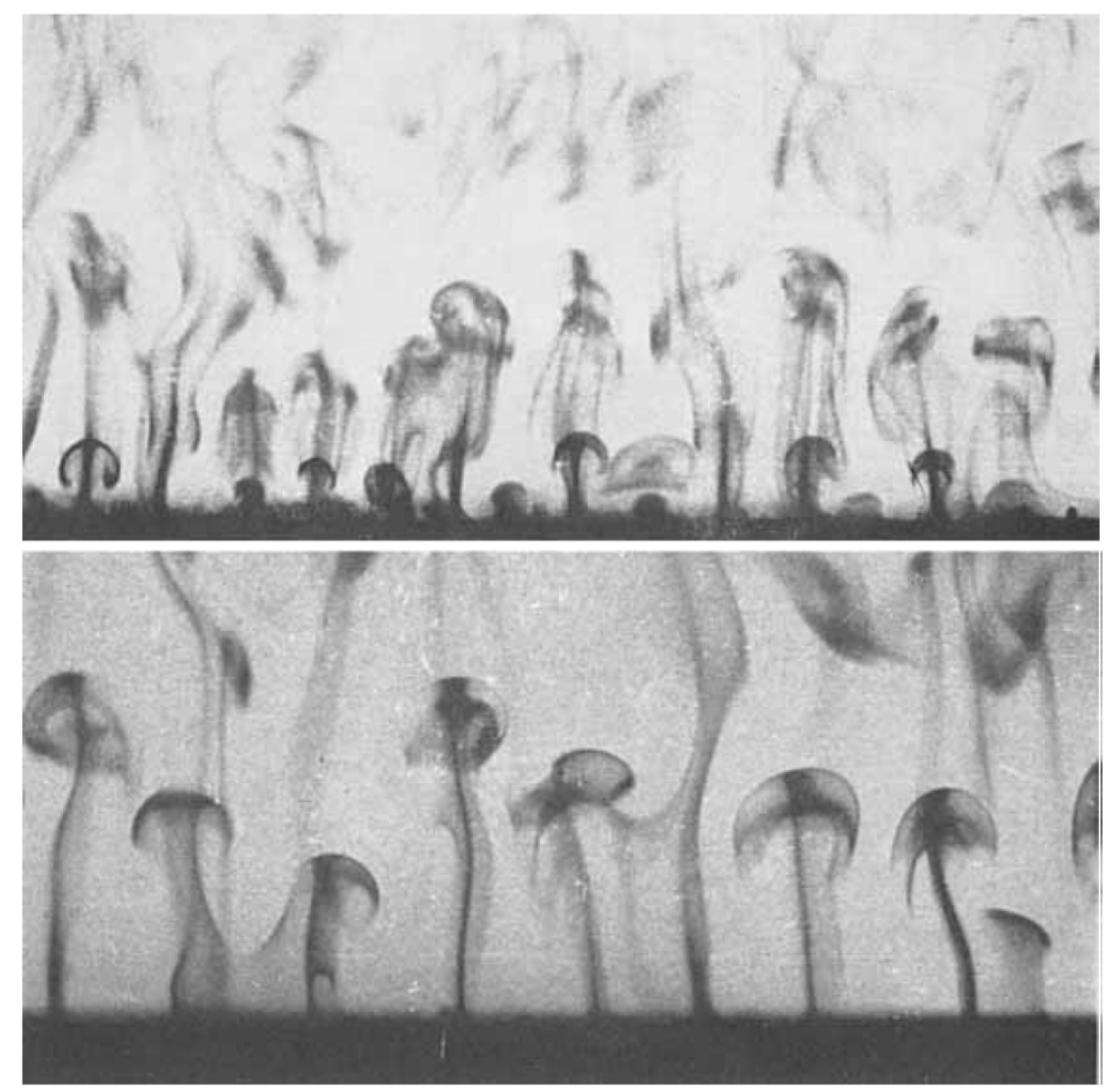

Figura 1.4: Estruturas Intermitentes de Fluido Aquecido numa Superfície Horizontal (Adaptado de (SPARROW; HUSAR; GOLDSTEIN, 1970))

Além das duas grandes categorias apresentadas, existe uma situação pouco explorada e que está associada ao processo de esterilização de produtos embalados. Essa configuração de problema consiste em aquecer todas as faces ao mesmo tempo e a mesma temperatura.

As interações entre os padrões de escoamento próximas da parede inferior aquecida com a zona de recirculação principal, é uma característica muito interessante deste tipo de escoamento. A quebra da estabilidade dinâmica e a simetria do escoamento durante o tempo de aquecimento será um campo de exploração nas simulações dos casos neste trabalho.

A convecção natural em uma cavidade paralelepípeda retangular, em que todas as paredes são aquecidas simultaneamente a uma temperatura $T_{W}$, apresenta características interessantes quanto à formação de padrões. Esses padrões são estruturas de bolhas 
de fluidos aquecidos que se assemelham às células de Bénard que surgem em ciclos, isoladamente ou em forma de matriz na parede inferior da cavidade. Compreender o processo de convecção e como fazer para acelerar o aquecimento da cavidade de um fluido newtoniano é o desafio desse trabalho.

O mesmo comportamento transitório é esperado para os casos de teste propostos no presente estudo, e algumas questões serão exploradas:

- Quais as características físicas do escoamento influenciam no aparecimento de recirculações internas?

- Quais estruturas do escoamento ocasionam a quebra de simetria?

- O estabelecimento da estratificação térmica está sincronizado com a formação da camada limite vertical?

As contribuições que o presente trabalho propõe apresentar são:

- Estudo sobre a caracterização da convecção natural em uma cavidade paralelepípeda retangular com todas as faces aquecidas aos mesmo tempo e a mesma temperatura - analisando os padrões formados no campo de temperatura (figura (1.5) ) à medida em que varia-se o número de Rayleigh e os aspectos que levam à quebra de simetria.

- Avaliar a imposição da agitação/rotação no processo de convecção natural no sentido de diminuir o tempo que se leva para o aquecimento da cavidade.

- Analisar o processo de transição laminar-turbulento para o problema proposto, através da conexão da técnica de Simulação de Grandes Escalas (SGE), em inglês Large Eddy Simulation (LES) para o problema de convecção natural na cavidade.

- Implementações numéricas no software de Fluidodinâmica Computacional (CFD - Computation Fluid Dynamics) OpenFOAM (Open Field Operation and Manipulation) - utilizando como base a estrutura de código aberto do OpenFOAM, será compilada todas as alterações necessárias para que possamos verificar os objetivos do trabalho que vai desde as imposições das condições de contorno, até a inserção da equação de energia no código de SGE. 
- A inovação do presente trabalho consiste do conjunto de resultados obtidos que caracterizam a convecção natural na cavidade cuja condições de contorno são pouco exploradas na literatura. Além disso, as implementações numéricas elaboradas no OpenFOAM servirão de ferramentas que podem ser utilizadas para outros tipos de geometrias

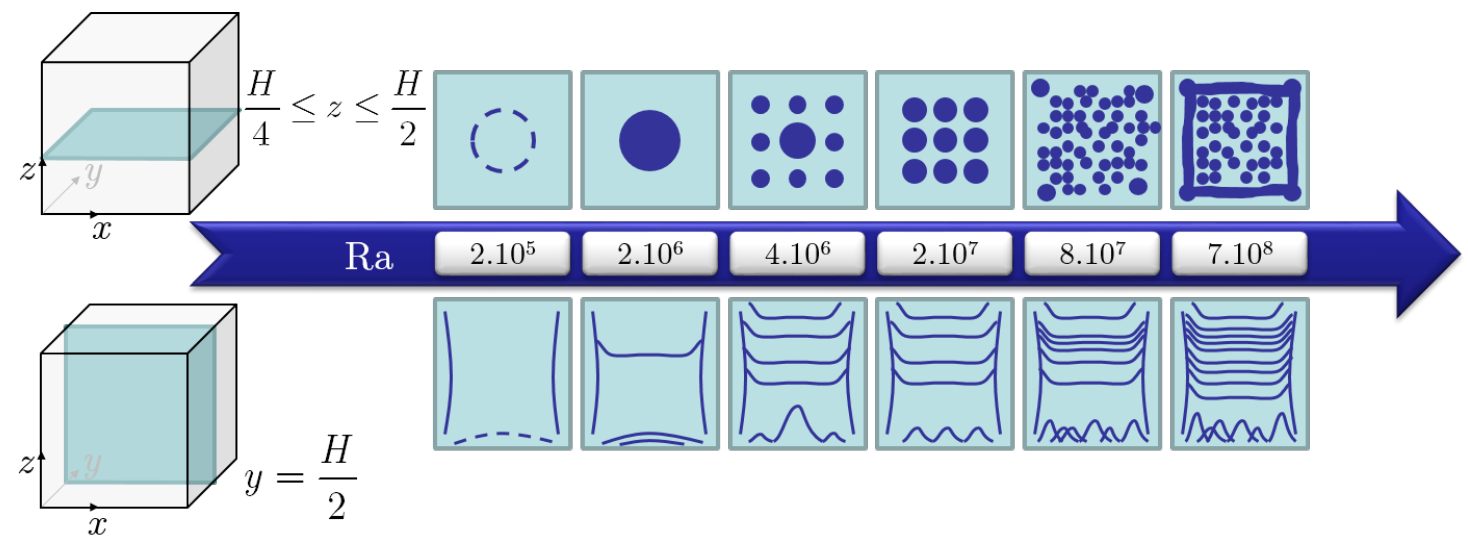

Figura 1.5: Características da Convecção Natural na Cavidade: Temperatura.

\subsection{Objetivos}

O presente trabalho tem como objetivo geral a modelagem e a simulação computacional para caracterização dos padrões do escoamento e perda de simetria da convecção natural laminar numa cavidade paralelepípeda retangular de um fluido newtoniano com um referencial fixo ou com agitação para vários números de Rayleigh. Para cumprir tal objetivo, algumas etapas terão que ser alcançadas e estão listadas a seguir:

- Estudo sobre a formulação matemática para a convecção natural laminar;

- Estudo da ferramenta de Fluidodinâmica Computacional, CFD, visando a implementação e extensão de códigos com a hipótese de Boussinesq;

- Desenvolvimento de metodologias e algoritmos para a simulação de escoamentos com agitação;

- Implementação das metodologias no pacote CFD; 
- Avaliação e análise dos resultados simulados.

O desenvolvimento deste trabalho deixa para a comunidade científica uma ferramenta acessível para simulação de convecção natural em uma cavidade que inclui a opção de agitação e/ou rotação para fluido newtoniano. O fato de ser desenvolvido em código aberto permite ainda a futura manipulação e possível aperfeiçoamento do código pelos perquisadores e usuários do OpenFOAM.

\subsection{Convecção Natural em Cavidades}

Muitos artigos relativos à convecção natural transiente em invólucros bidimensionais e tridimensionais são apresentados na literatura. (HSIEH; WAND, 1994) e (HSIEH; YANG, 1996) analisaram o problema tridimensional de uma cavidade aquecida de várias formas. Esses trabalhos apresentam a visualização do escoamento experimental e o campo transiente de temperatura. A história da temperatura e da visualização do escoamento durante o aquecimento mostrou um comportamento oscilatório inicial até a formação de uma zona de núcleo estável de recirculação e uma estratificação do campo térmico. Fusegi, Hyun e Kuwahara (1991) e Fusegi et al. (1991) aplicaram o método de diferenças finitas com esquema de precisão de terceira ordem para simular uma cavidade cúbica com ar para os números de Rayleigh de $10^{5}$ e $10^{6}$. Os efeitos específicos das condições de contorno térmicas horizontais sobre a estrutura de escoamento foram examinadas em detalhe. Foi constatado que a transferência de calor através das paredes horizontais melhoraram as atividades de fluxo convectivo. A velocidade numericamente prevista e perfis de temperatura nos planos de simetria são consistentes com as medições experimentais.

O trabalho experimental de Lin (1982) e Lin e Akins (1983) também analisa os padrões de escoamento e de transferência de calor em cavidades. Neste caso, a cavidade é cúbica e todas as paredes são subitamente aquecidas. O artigo mostra um grande número de padrões de fluxo que evoluem ao longo do tempo de aquecimento e são dependentes do número de Rayleigh. Seguindo a mesma linha de pesquisa, Tollini (1996) analisou a transferência de calor transiente e tridimensional em uma cavidade em forma de paralelepípedo, com razão de aspecto de $A_{h}=1,74$, com as mesmas dimensões de uma caixa de leite. Foram feitas comparações entre os parâmetros representativos da 
transferência de calor e a visualização da estrutura do escoamento convectivo, para valores de número de Rayleigh entre $1,08 \times 10^{9}$ e $8,24 \times 10^{9}$, experimentalmente e por simulação numérica. Estes dois estudos são semelhantes ao que será analisado no presente trabalho, conforme mostrado na Fig. (2.1).

O processo de convecção natural aplicado para o aquecimento de cavidades é muito utilizado na indústria de alimentos (HIDDINK, 1975), principalmente para o processo de conservação ou esterilização de produtos embalados ((AUGUSTO, 2009) e (AUGUSTO, 2012)). Em muitos casos há a necessidade de promover na cavidade a agitação com a finalidade de acelerar a transferência de calor entre o meio de aquecimento e a embalagem (GUMERATO, 2004). Uma grande parte desses alimentos são fluidos não newtonianos, onde a tensão de cisalhamento não é diretamente proporcional a taxa deformação ((CHHABRA; RICHARDSON, 2011)), modelos de transporte em que a viscosidade varie com a temperatura ((CHHABRA; RICHARDSON, 2011 apud HERSCHEL; BULKLEY, 1926) ) e com o tempo ((CHHABRA; RICHARDSON, 2011 apud FIGONI; SHOEMAKER, 1981)) (uma generalização do modelo de plástico de Bingham).

\subsection{Organização do Texto}

Este trabalho apresenta um estudo sobre a modelagem e a simulação computacional da convecção natural numa cavidade paralelepípeda retangular, obtidos pelo Método de Volumes Finitos e implementados no software de fluidodinâmica OpenFOAM (Open Field Operation and Manipulation). Dentro desse contexto alguns aspectos são parte do tema deste trabalho: $(i)$ verificação da capacidade do OpenFOAM em prever os resultados em comparação com dados experimentais na literatura; (ii) caracterização do surgimento de padrões no escoamento, assim como a análise da quebra de simetria; (iii) incorporação ao código do solver do OpenFOAM a agitação do domínio para observar a mudança no processo de aquecimento; e (iv) A análise do processo de transição laminar-turbulento. Na sequência, estão apresentadas a estrutura e o conteúdo dos capítulos deste trabalho.

O Capítulo 2 mostra a formulação matemática das equações de conservação que representam o processo de convecção natural para fluidos newtonianos: conservação de massa, de quantidade de movimento e de energia. E suas variações quando forças 
surgem devido ao processo de agitação da cavidade.

O pacote de fluidodinâmica computacional OpenFOAM de código livre usado neste trabalho é apresentado no Capítulo 3. Detalhes sobre a história e uso do software como ferramenta de fluidodinâmica são colocados. Uma breve introdução sobre a linguagem de programção $\mathrm{C}++$ é colocada para que o leitor tenha base para acompanhar a programação interna do OpenFOAM e a estrutura de bibliotecas usadas para manipulação e resolução de campos. Por fim, são colocados detalhes sobre a modelagem da convecção natural no OpenFOAM, considerando o fluido newtoniano para uma cavidade com domínio com referencial fixo ou móvel (agitação).

Os resultados deste trabalho são mostrados no Capítulo 4 que apresenta a comparação com dados experimentais dos trabalhos de (LIN, 1982), (LIN; AKINS, 1983) e (TOLLINI, 1996). Análises da evolução da convecção natural, assim como do aparecimento de padrões no escoamento, são caracterizados principalmente pela simetria e o instante de quebra desses padrões para diferentes números de Rayleigh. São avaliados a imposição de agitação na cavidade e seu efeito no processo de convecção para a aceleração do aquecimento do fluido no seu interior e sua evolução até o momento de transição laminar-turbulento.

Por fim, o Capítulo 5 apresenta os resultados da proposta de caracterizar a convecção na cavidade, comentando a evolução do código na implementação no OpenFOAM. 


\section{Capítulo 2}

\section{Formulação Matemática}

Este trabalho estuda o problema de convecção natural laminar e turbulenta em uma cavidade paralelepípeda retangular de lados $H, L$ e $W$, conforme a figura 2.1 .

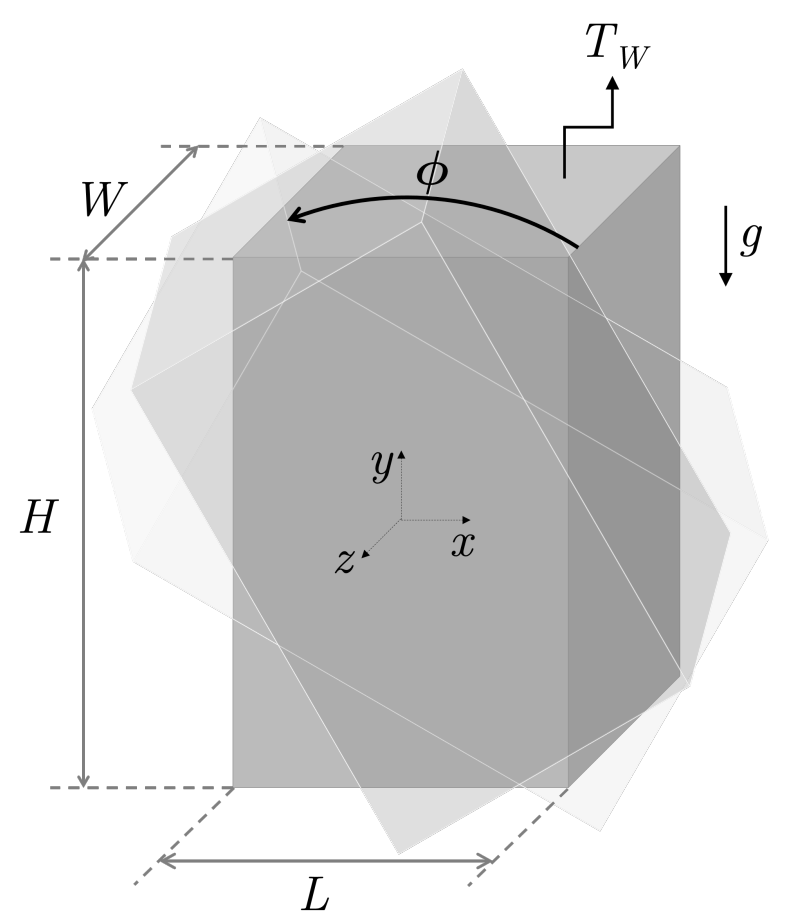

Figura 2.1: Cavidade paralelepípeda retangular

No seu interior, a cavidade está preenchida com fluido com massa específica $\rho$, viscosi- 
dade dinâmica $\nu$ e condutividade térmica $k$. A distribuição de temperatura no interior da cavidade inicialmente está a uma temperatura uniforme $T_{0}$, com o fluido em repouso. As seis paredes da cavidade são submetidas a uma temperatura $T_{W}$ maior que $T_{0}$ e a inclinação $\phi$ pode variar ao longo do tempo $t$. A convecção natural resultante aumenta consideravelmente a transferência de calor para a cavidade. Após um período de tempo suficiente, quando a temperatura da cavidade atinge a temperatura $T_{W}$, a convecção no seu interior cessa.

\subsection{Equações Governantes}

A modelagem matemática do problema de convecção natural envolve a equação de conservação de massa

$$
\frac{D \rho}{D t}+\rho \nabla \cdot \mathbf{u}=0
$$

de balanço da quantidade de movimento (Cauchy)

$$
\rho \frac{D \mathbf{u}}{D t}=\nabla \cdot \boldsymbol{\tau}-\rho \mathbf{g}+\rho \mathbf{a}
$$

e de conservação de energia:

$$
\rho C_{p} \frac{D(T)}{D t}=\nabla \cdot(k \nabla T)+\boldsymbol{\tau}: \nabla \mathbf{u}+q^{\prime \prime \prime}
$$

em que $\rho$ é a massa específica, $k$ é a condutividade térmica, $C_{P}$ é o calor específico, $\mathbf{u}(\mathbf{x}, t)$ o vetor velocidade euleriano, $\boldsymbol{\tau}$ o tensor de tensões e $\mathbf{g}$ uma força de campo conservativa (gravidade). O operador derivada material é definido por $D / D t=\partial / \partial t+\mathbf{u} \cdot \nabla$ e mede a taxa de variação temporal de uma quantidade qualquer do escoamento calculada a partir de um referencial que translada com uma partícula material. O divergente do tensor de tensões, $\nabla \boldsymbol{\tau}$, representa a taxa de quantidade de movimento por unidade de volume associada as forças de superfície, exercidas em um elemento de fluido, pelo material externo ao mesmo. A aceleração inercial, a, é devido ao movimento relativo do referencial do sistema em relação a um referencial inercial. Como os movimentos de translação e rotação entre referenciais são independentes, a aceleração inercial é dada em função da aceleração translacional, da aceleração angular, da aceleração de Coriolis 
e Centrífuga. O termo de dissipação viscosa, $\boldsymbol{\tau}: \nabla \mathbf{u}$, ou função de dissipação viscosa advém do desdobramento do trabalho de deformação do fluido. E, finalmente o termo fonte, $q^{\prime \prime \prime}$, que representa as fontes e sumidouros de energia térmica por unidade de volume.

Em nossas simulações consideraremos o tipo de fluido como sendo incompressível ea partir dessa hipótese, as equações 2.1, 2.2 e 2.3 podem ser reescritas:

$$
\begin{gathered}
\nabla \cdot \mathbf{u}=0 \\
\rho\left(\frac{\partial \mathbf{u}}{\partial t}+\mathbf{u} \cdot \nabla \mathbf{u}\right)=\nabla \boldsymbol{\tau}-\rho \mathbf{g}+\rho \mathbf{a}, \\
\frac{\partial T}{\partial t}+\mathbf{u} \cdot \nabla T=\alpha \nabla^{2} T+\boldsymbol{\tau}: \nabla \mathbf{u}+q^{\prime \prime \prime},
\end{gathered}
$$

sendo $\alpha=\frac{k}{\rho C_{p}}$ a difusividade térmica do fluido.

Como o movimento do fluido em convecção natural é impulsionado pela variação da densidade e o efeito da gravidade, a abordagem ao considerarmos $\rho=$ cte, não poderá ser feita em todos os termos das equações. Pois, há um fator que agrega complexidade à solução das equações: a variação inevitável da massa específica $(\rho)$ com a temperatura (T) (BEJAN; KRAUS, 2003). Várias aproximações são geralmente feitas para simplificar essas equações. Uma das mais importantes é a aproximação através da hipótese de Boussinesq ((ARPACI; LARSEN, 1984)).

$$
\rho=\rho_{0}\left[1-\beta\left(T-T_{0}\right)\right]
$$

onde $\beta$ é o coeficiente de expansão térmica a pressão constante.

A abordagem principal desta aproximação é de tratar a massa específica constante na equação de continuidade e no termo de inércia da equação de quantidade de movimento, mas permitir que a massa específica varie com a temperatura no termo da gravidade (empuxo), isto é, o efeito da pressão sobre a densidade é negligenciado. Na verdade, a diferença de densidade é calculada pela flutuação térmica. 
Substituindo a aproximação para $\rho$ (Eq. (2.7) ) na equação de conservação de quantidade de movimento (Eq. (2.5)), temos as novas equações

$$
\begin{gathered}
\nabla \cdot \mathbf{u}=0 \\
\rho\left(\frac{\partial \mathbf{u}}{\partial t}+\mathbf{u} \cdot \nabla \mathbf{u}\right)=\nabla \boldsymbol{\tau}-\rho_{0} \mathbf{g} \beta\left(T-T_{0}\right)+\rho \mathbf{a} \\
\frac{\partial T}{\partial t}+\mathbf{u} \cdot \nabla T=\alpha \nabla^{2} T
\end{gathered}
$$

Foram desprezados a dissipação viscosa e o trabalho realizado pelas forças de compressão. Além disso, nesse trabalho não consideraremos a geração interna de energia.

A próxima etapa na modelagem consiste na definição da equação constitutiva do tensor de tensões, $\boldsymbol{\tau}$, que será interpretado considerando o fluido newtoniano.

Para o problema envolvendo fluido newtoniano, em geral, o tensor de tensões pode ser decomposto em uma parte isotrópica (é todo aquele que pode ser escrito de forma mais geral como $c(\mathbf{x}, t) \mathbf{I}$, em que $c(\mathbf{x}, t)$ é um campo escalar) somado de uma parte deviatórica (é um tensor de traço nulo do tipo $\mathbf{T}^{(d)}=\mathbf{T}-\frac{1}{3} \operatorname{tr}(\mathbf{T}) \mathbf{I}$, onde $\mathbf{I}$ é o tensor identidade), ou seja,

$$
\boldsymbol{\tau}=-p \mathbf{I}-\mathbf{S}
$$

em que $p$ é a pressão mecânica, determinada por $p=-\frac{1}{3} \operatorname{tr}(\boldsymbol{\tau}) \mathrm{e}$

$$
\mathbf{S}=2 \mu\left[\mathbf{D}-\frac{1}{3}(\nabla \cdot \mathbf{u}) \mathbf{I}\right]
$$

em que $\mu$ é a viscosidade dinâmica e $\mathbf{D}$ o tensor taxa de deformação, definido como sendo a parte simétrica do tensor gradiente de velocidade dada por $\mathbf{D}=\frac{1}{2}\left(\nabla \mathbf{u}+\nabla \mathbf{u}^{T}\right)$. Considerando um fluido incompressível e substituindo a equação constitutiva 2.12 com a condição de incompressibilidade na equação 2.9, obtemos a equação de Navier-Stokes 


$$
\rho\left(\frac{\partial \mathbf{u}}{\partial t}+\mathbf{u} \cdot \nabla \mathbf{u}\right)=-\nabla p+\mu \nabla^{2} \mathbf{u}-\rho_{0} \mathbf{g} \beta\left(T-T_{0}\right)+\rho \mathbf{a} .
$$

\subsection{Modelagem da Turbulência}

O movimento turbulento do fluido é altamente aleatório, instável e tridimensional, que consiste de muitas estruturas com diferentes escalas de comprimento e tempo. Devido a estas complexidades, os movimentos turbulentos têm uma dificuldade extrema de serem descritos e então de serem previstos teoricamente.

Com a capacidade computacional atualmente disponível não é possível utilizar as equações instantâneas de Navier-Stokes para simular todas as escalas de tempo e espaço de um escoamento turbulento complexo. No entanto, uma solução com todos os detalhes, em todo o espectro de escalas de tempo, espaço e frequência de um escoamento turbulento é raramente necessária.

A necessidade de simplificações no tratamento de turbulência resultou ao longo dos anos em vários tipos de modelos, cada um deles resolvendo intervalos diferentes das escalas da turbulência.

Dentre estas abordagens, destacam-se a Simulação Numérica Direta (Direct Numerical Simulation - DNS), as Equações Médias de Reynolds (Reynolds Averaged Navier-Stokes - RANS) e a Simulação de Grandes Escalas (Large Eddy Simulation - LES).

Em DNS as Eqs. 2.8, 2.10 e 2.13) são resolvidas sem modelagem, em malhas bastante refinadas com passos de tempo bem pequenos, a fim de capturar toda a gama de escalas turbulentas.

As equações da técnica RANS são obtidas através de um conjunto de médias das equações 2.8), 2.10) e (2.13). O elemento crítico da modelagem RANS é a representação das tensões de Reynolds ou tensões turbulentas.

Na técnica de LES, as grandes escalas, consideradas como turbilhões que contém energia, são calculadas diretamente e, para as pequenas escalas, utilizam-se modelos de 
escalas sub-malha.

Na Fig. 2.2 são comparados o processo de filtragem que cada modelo aproxima sobre uma malha computacional para dados de velocidade em função do tempo.

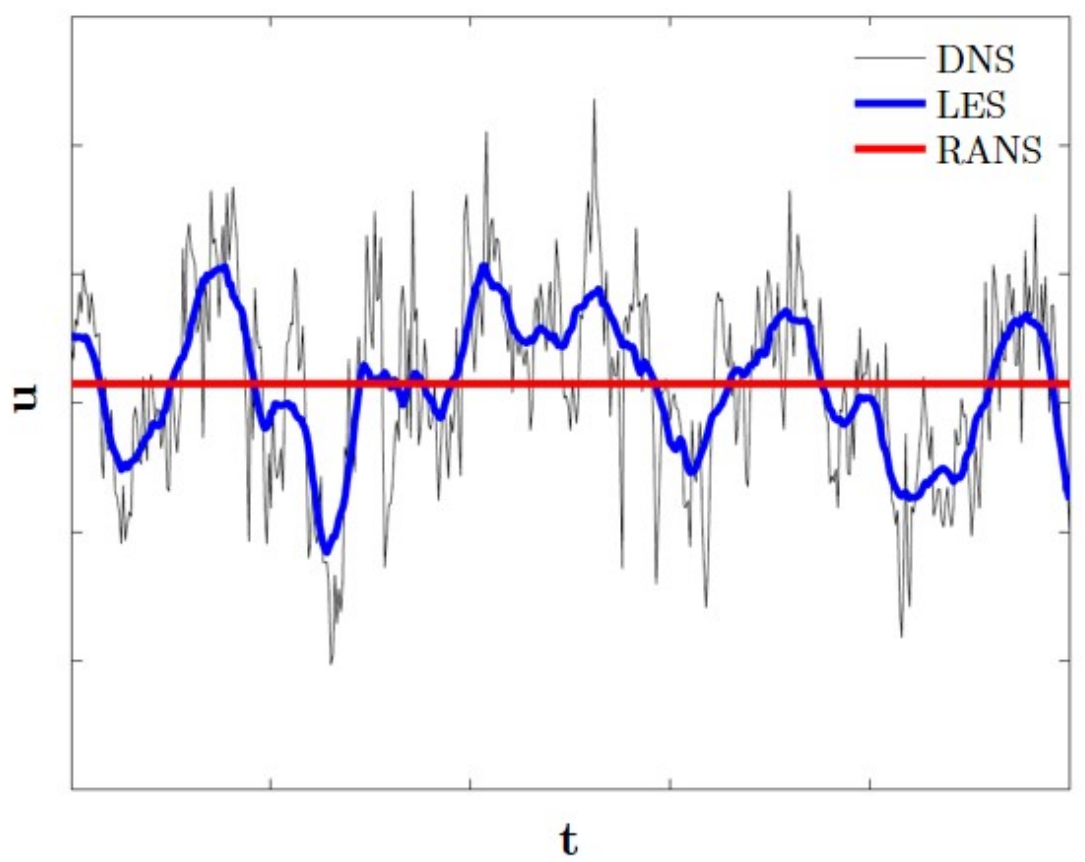

Figura 2.2: Evolução temporal da velocidade calculada por DNS, LES e RANS em um escoamento turbulento (Adaptado de (STOLL, 2014)).

\subsubsection{Decomposição de Reynolds}

A solução de valores médios que possam servir de parâmetros para o estudo do caso é implementada. Desta forma é desenvolvido um método estatístico para a solução do problema médio do escoamento, baseado na tomada da média das equações instantâneas de Navier-Stokes. As equações médias resultantes deste procedimento são chamadas de equações médias de Reynolds. Através deste método é possível avaliar os valores médios e desvios padrão das variáveis do escoamento, o que é informação suficiente para a solução de uma grande gama de problemas. Por meio de valores médios procura-se reduzir o espectro de escalas necessárias na simulação do escoamento turbulento. O custo computacional reduzido da utilização das equações médias permite a simulação de escoamentos complexos nos computadores disponíveis em larga escala. 
No procedimento de tomada de média de Reynolds das equações de Navier-Stokes é tirada a média sobre um período de tempo maior que o período de tempo característico do movimento do escoamento. As variáveis físicas são decompostas em componentes médias e de flutuação. Somente os valores médio são resolvidos e conseqüentemente é necessário expressar os valores de flutuação em função destes. As equações resultantes descrevem o campo médio de velocidade.

As variáveis instantâneas $\varphi$ são decompostas como segue:

$$
\varphi(x, t)=\bar{\varphi}(x)+\varphi^{\prime}(x, t)
$$

onde $\bar{\varphi}$ é o valor médio da variável e $\varphi^{\prime}$ é variação instantânea (flutuação) da variável.

O operador de média temporal é definido como:

$$
\bar{\varphi}=\lim _{\Delta t \rightarrow \infty} \frac{1}{\Delta t} \int_{t_{0}}^{t_{0}+\Delta t} \varphi d t=\bar{\varphi}(x),
$$

onde o intervalo de tempo $\Delta t$ é maior que a escala de tempo das flutuações turbulentas, de forma que seja grande suficiente se comparado com o período das flutuações e que seja pequeno o suficiente com relação à constante de tempo das variações no campo do escoamento.

As equações médias são conseguidas através da substituição da decomposição de Reynolds nas equações de conservação e da tomada de média das equações resultantes.

\section{Observação:}

Na tomada das equações instantâneas deve-se lembrar:

- média da variação instantânea da velocidade é zero, ou seja:

$$
\overline{\varphi^{\prime}}=0
$$

- a média das variáveis médias é a própria variável média, desta maneira:

$$
\bar{\varphi}=\bar{\varphi} ;
$$


- e também:

$$
\overline{\bar{\varphi} \varphi^{\prime}}=\bar{\varphi} \overline{\varphi^{\prime}}=0
$$

- quando se aplica a média sobre termos não-lineares $(\nabla \cdot(\mathbf{u} \otimes \mathbf{u}))$ da equação de Navier-Stokes, aparecem termos de covariância das variáveis instantâneas, que devem ser analisados da seguinte maneira:

$$
\begin{gathered}
a=a+a^{\prime} ; \\
b=b+b^{\prime} ; \\
\overline{a b}=\overline{\left(a+a^{\prime}\right)\left(b+b^{\prime}\right)}=\overline{a b+a^{\prime} b+a b^{\prime}+a^{\prime} b^{\prime}}=\bar{a} \bar{b}+\overline{a^{\prime} b^{\prime} .}
\end{gathered}
$$

A decomposição de Reynolds para as equações de Navier-Stokes (para o escoamento incompressível) é dada por:

$$
\begin{aligned}
& \mathbf{u}=\mathbf{U}+\mathbf{u}^{\prime} \\
& T=\mathbf{T}+\mathbf{T}^{\prime} \\
& p=P+p^{\prime},
\end{aligned}
$$

sendo que a variável em maiúsculo é a componente média e com o símbolo (') é a componente instantânea.

Substituindo a decomposição de Reynolds nas equações incompressíveis:

$$
\begin{gathered}
\nabla \cdot\left(\mathbf{U}+\mathbf{u}^{\prime}\right)=0 \\
\frac{\partial\left(\mathbf{U}+\mathbf{u}^{\prime}\right)}{\partial t}+\nabla \cdot\left[\left(\mathbf{U}+\mathbf{u}^{\prime}\right) \otimes\left(\mathbf{U}+\mathbf{u}^{\prime}\right)\right]=-\frac{1}{\rho} \nabla\left(P+p^{\prime}\right)+\nabla \cdot\left(\overline{\mathcal{T}}+\mathcal{T}^{\prime}\right),
\end{gathered}
$$

ou, expandindo os termos em parênteses:

$$
\nabla \cdot \mathbf{U}+\nabla \cdot \mathbf{u}^{\prime}=0
$$


$\frac{\partial \mathbf{U}}{\partial t}+\frac{\partial \mathbf{u}^{\prime}}{\partial t}+\nabla \cdot\left(\mathbf{U} \otimes \mathbf{U}+\mathbf{U} \otimes \mathbf{u}^{\prime}+\mathbf{u}^{\prime} \otimes \mathbf{U}+\mathbf{u}^{\prime} \otimes \mathbf{u}^{\prime}\right)=-\frac{1}{\rho}\left(\nabla P+\nabla p^{\prime}\right)+\nabla \cdot \overline{\mathcal{T}}+\nabla \cdot \mathcal{T}^{\prime}$

e tomando a média, lembrando que a derivada comuta com o operador média (ambos são lineares), consegue-se o seguinte conjunto de equações médias:

$$
\begin{gathered}
\nabla \cdot \mathbf{U}=0 \\
\frac{\partial \mathbf{U}}{\partial t}+(\nabla \mathbf{U}) \mathbf{U}=-\frac{1}{\rho} \nabla P+\nabla \cdot(\nu \cdot \nabla \mathbf{U})-\nabla \cdot\left(\overline{\mathbf{u}^{\prime} \otimes \mathbf{u}^{\prime}}\right) .
\end{gathered}
$$

Analogamente ao processo de decomposição de Reynolds aplicado às equações de Navier-Stokes, a equação de energia torna-se:

$$
\frac{\partial \mathbf{T}}{\partial t}+\mathbf{U} \cdot \nabla \mathbf{T}=\alpha \nabla^{2} \mathbf{T}-\rho C_{p} \nabla\left(\overline{\mathbf{u}^{\prime} \mathbf{T}}\right)
$$

As equações resultantes descrevem o campo de escoamento médio. As equações médias são idênticas às equações instantâneas com a adição dos termos de correlação. O termo $\overline{\mathbf{u}^{\prime} \otimes \mathbf{u}^{\prime}}$ é conhecido como tensor de Reynolds $(\tau)$, e representa o efeito das flutuações turbulentas sobre a velocidade. Devido à natureza da turbulência é impossível modelar este termo de uma forma geral. Como o tensor de Reynolds é simétrico, o processo de tomada de média acrescenta seis (6) incógnitas adicionais no caso tridimensional e três (3) incógnitas adicionais no caso bidimensional.

\subsubsection{Problema de Fechamento}

Através da seção anterior verificamos que o processo de decomposição de Reynolds faz com que fiquemos com nove (9) incógnitas e apenas três (3) equações para a resolução do sistema de equações diferenciais, que não é linear devido ao termo convectivo que aparece na equação da conservação da quantidade de movimento (Eq. 2.26). 
Para resolver o problema de fechamento das equações de Reynolds, é necessário que as correlações turbulentas que compõem o tensor de Reynolds sejam modeladas.

As soluções propostas para a modelagem do tensor de Reynolds podem ser de natureza constitutiva, ou seja, uma formulação analítica - algébrica - do tensor de Reynolds.

A primeira tentativa de modelação do tensor de Reynolds é devida a Joseph Boussinesq (1842-1929) e apesar de fundamentada em conceitos incorretos, a hipótese de Boussinesq continua como ponto de partida para a maior parte dos modelos de turbulência empregados atualmente pela dinâmica dos fluidos computacional.

Devido ao surgimento de um coeficiente de atrito suplementar, originado pelo regime turbulento e independente do coeficiente de atrito existente no regime laminar original. Este coeficiente, denominado por Boussinesq como atrito interno, se apresenta como uma grandeza escalar que depende linearmente dos gradientes de velocidade média representativos do escoamento turbulento e que independe da pressão e da temperatura na qual o escoamento acontece.

Na hipótese de Boussinesq o campo de tensões provocado pelo "atrito interno", é função de uma viscosidade dinâmica turbulenta hipotética $\nu_{t}$, a ser determinada, e dos gradientes de velocidade média do escoamento turbulento. A generalização da hipótese de Boussinesq, proposta por Kolmogorov em 1942 ((POPE, 2000) $)$, tem como representação a expressão:

$$
-\overline{\mathbf{u}^{\prime} \otimes \mathbf{u}^{\prime}}=2 \nu_{t} \mathbf{S}(\mathbf{u})-\frac{2}{3}\left[\nu_{t}(\nabla \cdot \mathbf{u})+k I\right]
$$

Para o nosso caso onde a massa específica é constante $(\nabla \cdot \mathbf{u}=0)$ a expressão se reduz para:

$$
-\overline{\mathbf{u}^{\prime} \otimes \mathbf{u}^{\prime}}=2 \nu_{t} \mathbf{S}(\mathbf{u})-\frac{2}{3}(k I)
$$

Ao longo das últimas décadas uma grande diversidade de modelos de turbulência têm sido propostos, isto devido ao avanço no campo computacional proporcionando a criação e simulação de modelos mais complexos. 
Os modelos que iremos trabalhar estão centrados na hipótese de Boussinesq, que são classificados como:

- Modelos a uma equação - Modelos capazes de representar a viscosidade turbulenta, $\nu_{t}$, a partir de uma equação diferencial parcial;

- Modelos a duas equações - Modelos capazes de representar a viscosidade turbulenta, $\nu_{t}$, a partir de duas equações diferenciais parciais.

\subsubsection{Modelos a Duas Equações}

As equações das componentes flutuantes são obtidas subtraindo as equações médias (Eq. 2.23 e Eq. 2.24) das equações instantâneas (Eq. 2.25 e Eq. 2.26). A equação da continuidade para as parcelas flutuantes da velocidade é dada por:

$$
\left(\nabla \cdot \mathbf{U}+\nabla \cdot \mathbf{u}^{\prime}=0\right)-(\nabla \cdot \mathbf{U}=0)=\left(\nabla \cdot \mathbf{u}^{\prime}=0\right)
$$

Enquanto que para a equação de Navier-Stokes para as parcelas flutuantes da velocidade é dada por:

$$
\begin{gathered}
\frac{\partial \mathbf{U}}{\partial t}+\frac{\partial \mathbf{u}^{\prime}}{\partial t}+\nabla \cdot\left(\mathbf{U} \otimes \mathbf{U}+\mathbf{U} \otimes \mathbf{u}^{\prime}+\mathbf{u}^{\prime} \otimes \mathbf{U}+\mathbf{u}^{\prime} \otimes \mathbf{u}^{\prime}\right)= \\
-\frac{1}{\rho}\left(\nabla P-\nabla p^{\prime}\right)+\nabla \cdot(\nu \nabla \mathbf{U})+\nabla \cdot\left(\nu \nabla \mathbf{u}^{\prime}\right) \\
-\left[\frac{\partial \mathbf{U}}{\partial t}+\nabla \cdot(\mathbf{U} \otimes \mathbf{U})=-\frac{1}{\rho}(\nabla P+\nabla \cdot \bar{\tau})-\nabla \cdot\left(\overline{\mathbf{u}^{\prime} \otimes \mathbf{u}^{\prime}}\right)\right]= \\
=\frac{\partial \mathbf{u}^{\prime}}{\partial t}+\nabla \cdot\left(\mathbf{U} \otimes \mathbf{u}^{\prime}+\mathbf{u}^{\prime} \otimes \mathbf{U}+\mathbf{u}^{\prime} \otimes \mathbf{u}^{\prime}\right)=-\frac{1}{\rho} \nabla p^{\prime}+\nabla \cdot\left(\nu \nabla \mathbf{u}^{\prime}\right)+\nabla \cdot\left(\overline{\mathbf{u}^{\prime} \otimes \mathbf{u}^{\prime}}\right) .
\end{gathered}
$$

A Eq. 2.29 para as parcelas flutuantes serve como base para a equação de transporte do tensor de Reynolds. A equação evolutiva para o tensor de Reynolds é obtida multiplicando-se a Eq. 2.29 por $u^{\prime}$ e tirando-se a média, cujo resultado é a equação: 


$$
\left[\overline{\frac{\partial \mathbf{u}^{\prime}}{\partial t}+\nabla \cdot\left(\mathbf{U} \otimes \mathbf{u}^{\prime}+\mathbf{u}^{\prime} \otimes \mathbf{U}+\mathbf{u}^{\prime} \otimes \mathbf{u}^{\prime}\right)=-\frac{1}{\rho} \nabla P+\nabla \cdot\left(\nu \nabla \mathbf{u}^{\prime}\right)+\nabla \cdot\left(\overline{\mathbf{u}^{\prime} \otimes \mathbf{u}^{\prime}}\right)}\right] \cdot \mathbf{u}^{\prime},
$$

e somando a Eq. 2.32 a ela mesma, temos a equação:

$$
\begin{gathered}
\frac{\partial\left(\overline{\mathbf{u}^{\prime} \otimes \mathbf{u}^{\prime}}\right)}{\partial t}+\mathbf{U} \cdot \nabla\left(\overline{\overline{\mathbf{u}^{\prime} \otimes} \mathbf{u}^{\prime}}\right)=\nabla \cdot\left(\overline{\overline{\mathbf{u}^{\prime} \otimes \mathbf{u}^{\prime} \otimes \mathbf{u}^{\prime}}}\right)-\frac{1}{\rho}\left(\nabla \overline{p^{\prime} \mathbf{u}^{\prime}}+\nabla^{T} \overline{p^{\prime} \mathbf{u}^{\prime}}\right)+ \\
\left.\nabla \cdot\left[\nu \nabla\left(\overline{{\overline{u^{\prime}} \otimes \mathbf{u}^{\prime}}^{\prime}}\right)\right]-\left(\overline{\mathbf{u}^{\prime} \otimes \mathbf{u}^{\prime}}\right)\left(\nabla \mathbf{U}+\nabla^{T} \mathbf{U}\right)-2 \nu \overline{\nabla \mathbf{u}^{\prime} \cdot \nabla^{T} \mathbf{u}^{\prime}}+\frac{1}{\rho} \overline{p^{\prime}\left(\nabla \mathbf{u}^{\prime}+\nabla^{T} \mathbf{u}^{\prime}\right.}\right) .
\end{gathered}
$$

A equação da energia cinética de turbulência pode ser obtida a partir do traço da Eq. 1.25, lembrando que energia cinética de turbulência $k$ é definida através da equação:

$$
\operatorname{tr}\left(\overline{\mathbf{u}^{\prime} \otimes \mathbf{u}^{\prime}}\right) \equiv k=\frac{\overline{\mathbf{u}^{\prime} \cdot \mathbf{u}^{\prime}}}{2}
$$

A equação exata da energia cinética de turbulência é:

$$
\frac{\partial k}{\partial t}+\mathbf{U} \cdot \nabla k=\nabla \cdot\left(\nu \nabla k-\overline{\mathbf{u}^{\prime} k}-\frac{1}{\rho} \overline{p^{\prime} \mathbf{u}^{\prime}}\right)-\tau: \nabla \mathbf{U}-2 \nu\left(\overline{\nabla \mathbf{u}^{\prime}: \nabla \mathbf{u}^{\prime}}\right) .
$$

A equação pode ser reescrita da seguinte maneira:

$$
\frac{\partial k}{\partial t}+\mathbf{U} \cdot \nabla k=\nabla\left(D_{t k}+D_{m k}\right)-P_{k}-\varepsilon
$$

Os termos da Eq. 2.36 representam:

- 1. $\frac{\partial k}{\partial t}$ - Variação temporal local de $k$;

- 2. U $\cdot \nabla k-$ Transporte convectivo de $k$; 
- 3. $D_{t k}$ - Difusão turbulenta de $k$ - representa a taxa na qual a energia cinética turbulenta é difundida no fluido pelas flutuações turbulentas de pressão e velocidade;

- 4. $D_{m k}$ - Difusão molecular de $k$ - representa a difusão da energia cinética turbulenta por transporte molecular;

- 5. $P_{k}$ - Produção de $k$ - indica a taxa de transferência de energia cinética do escoamento médio para a turbulência. Esta transferência é causada pelo trabalho efetuado pela taxa de deformação média sobre as tensões de Reynolds;

- 6. $\varepsilon$ - Taxa de dissipação de $k$ - é a taxa na qual a energia cinética turbulenta é transformada em energia interna. Esta transferência de energia é causada pelo trabalho exercido pela taxa de deformação flutuante contra as tensões viscosas flutuantes.

Os termos (1), (2) e (4) são conhecidos, mas os termos restantes precisam ser modelados, já que contêm correlações desconhecidas. O termo (6) pode ser modelado através de uma equação adicional para a dissipação.

Os dois termos aparecendo em

$$
D_{t k}=-\left(\overline{\mathbf{u}^{\prime} k}+\frac{1}{\rho} \overline{p^{\prime} \mathbf{u}^{\prime}}\right)
$$

são associados ao transporte difusivo turbulento e são portanto aproximados através da ideia do conceito de viscosidade turbulenta:

$$
-\left(\overline{\mathbf{u}^{\prime} k}+\frac{1}{\rho} \overline{p^{\prime} \mathbf{u}^{\prime}}\right) \sim \gamma_{k} \nabla k
$$

A difusividade é determinada da analogia de Reynolds, que relaciona a difusividade de qualquer propriedade linearmente com a difusividade da quantidade de movimento, isto é:

$$
\gamma_{k} \sim \frac{\nu_{t}}{\sigma_{k}}
$$

onde $\sigma_{k}$ é o número de Prandtl turbulento para o transporte de $k$.

O termo $P_{k}$, onde aparece o tensor de Reynolds, pode ser aproximado utilizando a hipótese de Boussinesq (Eq. 2.36). 
Para o último termo, $\varepsilon$, o termo que se refere à taxa de dissipação viscosa de $\mathrm{k}$, utilizamos como aproximação a hipótese de equilíbrio local e a observação experimental, que relacionam a dissipação de energia dos grandes vórtices $(k)$ com suas escalas de tempo $\left(L / k^{1 / 2}\right)$, que produzem a seguinte estimativa para $\varepsilon$ :

$$
\varepsilon=2 \nu\left(\overline{\nabla \mathbf{u}^{\prime}: \nabla \mathbf{u}^{\prime}}\right) \sim \frac{k^{3 / 2}}{L_{\varepsilon}}
$$

Utilizando essas aproximações, o modelo a uma equação pode ser expresso como:

$$
\frac{\partial k}{\partial t}+\mathbf{U} \cdot \nabla k=\nabla \cdot\left[\left(\nu+\frac{\nu_{t}}{\sigma_{k}}\right) \nabla k\right]+\tau: \nabla \mathbf{U}-\frac{k^{3 / 2}}{L_{\varepsilon}}
$$

A viscosidade turbulenta é obtida da seguinte relação, proposta independentemente por Kolmogorov (1942) e Prandtl (1945) e caracterizada, em adição à velocidade $u=k^{1 / 2}$, pela escala de comprimento $L_{\mu}$ :

$$
\nu_{t}=C_{\mu} k^{\frac{1}{2}} L_{\mu}
$$

onde $C_{\mu}=0,09$ é uma constante empírica.

O ponto inicial para praticamente todos os modelos a duas equações é a aproximação de Boussinesq, Eq. 2.28 e a equação da energia cinética de turbulência na forma da Eq. 2.41

O primeiro passo para a resolução do problema de fechamento através de modelo de duas equações ou de segunda ordem é a derivação da equação de transporte do tensor de Reynolds. Esta equação pode ser obtida a partir das equações das componentes flutuantes da decomposição de Reynolds. A equação exata para a dissipação de energia cinética da turbulência pode ser obtida encontrando o gradiente $\nabla$ da Eq. 1.22, multiplicando esta equação resultante por $2 \nu \nabla \mathbf{u}^{\prime}$ e tomando a média do resultado da multiplicação:

$2 \nu \nabla\left(\mathbf{u}^{\prime}\right) \nabla\left[\frac{\partial \mathbf{u}^{\prime}}{\partial t}+\nabla \cdot\left(\mathbf{U} \otimes \mathbf{u}^{\prime}+\mathbf{u}^{\prime} \otimes \mathbf{U}+\mathbf{u}^{\prime} \otimes \mathbf{u}^{\prime}\right)=-\frac{1}{\rho} \nabla P+\nabla \cdot\left(\nu \nabla \mathbf{u}^{\prime}\right)+\nabla \cdot\left(\overline{\mathbf{u}^{\prime} \otimes \mathbf{u}^{\prime}}\right)\right]$. 
O resultado desta operação é a equação abaixo:

$$
\begin{gathered}
\frac{\partial \varepsilon}{\partial t}+\nabla(\mathbf{U} \varepsilon)=\nabla\left[\overline{\mathbf{u}^{\prime} \varepsilon^{\prime}}-2 \nu\left(\overline{\nabla \mathbf{u}^{\prime} \nabla p^{\prime}}\right)+\nu \nabla \varepsilon\right]-2 \nu \overline{\mathbf{u}^{\prime} \nabla \mathbf{u}^{\prime}} \nabla^{2} \mathbf{U}- \\
2 \nu \nabla \mathbf{U}\left[\overline{\nabla \mathbf{u}^{\prime} \nabla \mathbf{u}^{\prime}}+\overline{\nabla^{T} \mathbf{u}^{\prime} \nabla^{T} \mathbf{u}^{\prime}}\right]-2 \nu \overline{\nabla \mathbf{u}^{\prime} \nabla \mathbf{u}^{\prime} \nabla \mathbf{u}^{\prime}}-2 \nu^{2}{\overline{\left(\nabla^{2} \mathbf{u}^{\prime}\right)}}^{2} .
\end{gathered}
$$

A Eq. 1.31 pode ser reescrita como:

$$
\frac{\partial \varepsilon}{\partial t}+\nabla(\mathbf{U} \varepsilon)=\nabla\left(D_{t \varepsilon}+D_{m \varepsilon}\right)-P_{\varepsilon}-\Lambda
$$

Em que os termos da equação têm o seguinte significado:

- 1. $\frac{\partial \varepsilon}{\partial t}-$ Variação temporal local de $\varepsilon$;

- 2. $\nabla(\mathbf{U} \varepsilon)-$ Transporte convectivo de $\varepsilon$;

- 3. $D_{t \varepsilon}-$ Difusão turbulenta de $\varepsilon$;

- 4. $D_{m \varepsilon}-$ Difusão molecular de $\varepsilon$;

- 5. $P_{\varepsilon}-$ Produção de $\varepsilon$;

- 6. $\Lambda$ - Dissipação de $\varepsilon$.

Os termos (3), (5) e (6) contêm correlações desconhecidas e devem ser modelados para utilizar $\epsilon$ no cálculo do tensor de Reynolds.

\subsubsection{O Modelo $k-\varepsilon$}

O modelo $k-\varepsilon$ é o mais amplamente utilizado entre os modelos de turbulência e que se encontra incorporado na maioria dos códigos comerciais em CFD. Pertence à classe dos modelos a duas equações, em que as equações de transporte do modelo são resolvidas por duas quantidades turbulentas, isto é, $k$ e $\varepsilon$. Por estas quantidades pode 
ser formada uma escala de comprimento $\left(L=k^{3 / 2} / \varepsilon\right)$, uma escala de tempo $(\tau=k / \varepsilon)$ ou uma quantidade de dimensão $\nu_{t}\left(k^{2} / \varepsilon\right)((\mathrm{POPE}, 2000))$.

Em complemento à hipótese de viscosidade turbulenta, o modelo $k-\varepsilon$ consiste de:

1. Especificação da Viscosidade Turbulenta:

$$
\nu_{t}=C_{\mu} \frac{k^{2}}{\varepsilon}
$$

2. Equação de Transporte para a Energia Cinética de Turbulência $k$, Eq. (2.41):

$$
\frac{\partial k}{\partial t}+\mathbf{U} \cdot \nabla k=\nabla \cdot\left[\left(\nu+\frac{\nu_{t}}{\sigma_{k}}\right) \nabla k\right]+\tau: \nabla \mathbf{U}-\varepsilon
$$

3. Equação de Transporte para a Taxa de Dissipação, $\varepsilon$ :

$$
\frac{\partial \varepsilon}{\partial t}+\mathbf{U} \cdot \nabla \varepsilon=\nabla \cdot\left[\left(\nu+\frac{\nu_{t}}{\sigma_{\varepsilon}}\right) \nabla \varepsilon\right]+C_{\varepsilon 1} \frac{\epsilon}{k} \tau: \nabla \mathbf{U}-C_{\varepsilon 2} \frac{\varepsilon^{2}}{k}
$$

onde coeficientes de fechamento são:

$$
C_{\varepsilon 1}=1,44, \quad C_{\varepsilon 2}=1,92, \quad C_{\mu}=0,09, \quad \sigma_{k}=1,0 \quad \text { e } \quad \sigma_{\varepsilon}=1,3 .
$$

\subsubsection{Simulação de Grandes Escalas - SGE}

A Simulação de Grandes Escalas (SGE), em inglês Large Eddy Simulation (LES), é uma das mais promissoras metodologias para a solução de escoamentos turbulentos. A metodologia da SGE surgiu com os estudos do meteorologista ( SMAGORINSKY, 1963)). Com base na teoria de Kolmogorov (KOLMOGOROV, 1991), a ideia do pesquisador era de simular as grandes escalas dos escoamentos atmosféricos, visto que não era possível a obtenção de todo o espectro de escalas do escoamento. A SGE consiste na simulação direta das estruturas das grandes escalas, onde se encontram as frequências baixas do escoamento, as quais são produzidas pelas instabilidades do escoamento médio. As estruturas das grandes escalas são responsáveis pela maioria do transporte de movimento e de massa, sendo estas mais anisotrópicas e diferentes para cada tipo de escoamento. Por outro lado, as estruturas de pequenas escalas, onde se encontram 
as frequências altas do escoamento, são de menor importância para o escoamento, pois contribuem pouco para o transporte de movimento e calor. Portanto, a SGE, simula diretamente as grandes estruturas e modela as pequenas estruturas do escoamento.

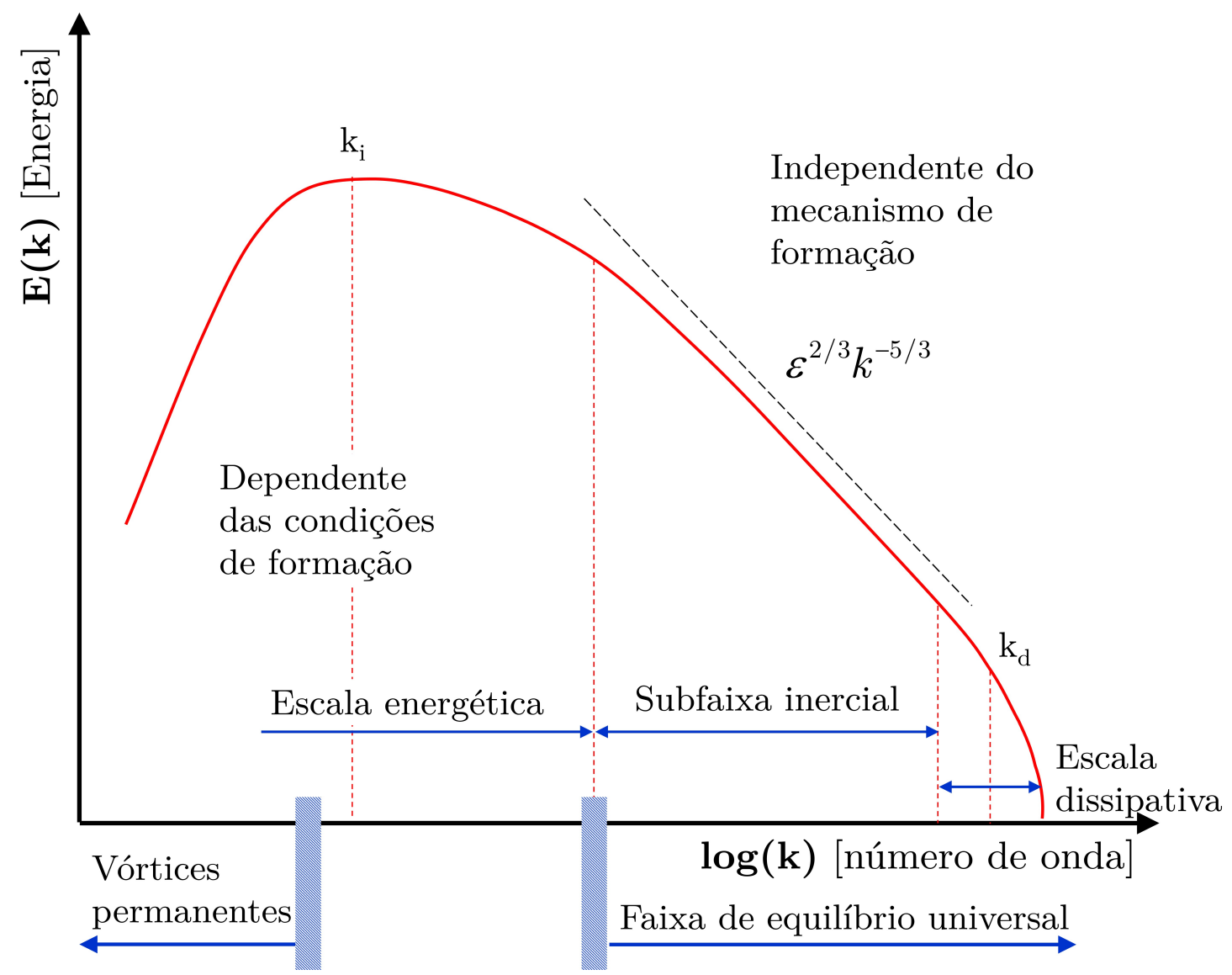

Figura 2.3: Espectro de Energia do Escoamento Turbulento (Adaptado de ((VILLIERS, 2006))

Na Simulação de Grandes Escalas, as equações de conservação do escoamento para as grandes escalas são obtidas após a aplicação de um filtro espacial sobre as equações da conservação de massa, da quantidade de movimento e da energia. Como resultado, são obtidas tensões adicionais, termos esses que não resolvidos pela malha. Estas tensões adicionais, além do tensor viscoso, devem ser modeladas empregando-se os modelos de aproximação existentes.

Semelhante à modelagem que utilizou a média de Reynolds, os escalares são decompostos em duas parcelas, porém estas não estão separadas pelo comportamento temporal, e sim por uma restrição definida pela dimensão característica do filtro. Nesta aproximação é realizado primeiramente uma decomposição das variáveis de campo de grandes 
escalas $\bar{\phi}$ e de campo submalha $\phi^{\prime}$, sendo:

$$
\phi(\mathbf{x}, t)=\bar{\phi}(\mathbf{x}, t)+\phi^{\prime}(\mathbf{x}, t)
$$

onde $\phi$ pode representar o campo de velocidade $\mathbf{u}$, a pressão estática do fluido $p$, a massa específica $\rho$ ou o campo de temperatura $T$

O processo de filtragem das variáveis $\phi$ é feito para se obter o campo de grandes escalas do escoamento. A componente $\bar{\phi}$ é definida, conforme Leonard (LEONARD, 1975), como sendo a convolução entre a variável $\phi$ com uma função de filtro $G(\mathbf{x})$ no domínio $\Omega$, sendo dada por

$$
\bar{\phi}(\mathbf{x}, t)=\oint_{\Omega} G\left(\mathbf{x}-\mathbf{x}^{\prime}\right) \phi(\mathbf{x}, t) d \mathbf{x}^{\prime},
$$

Um filtro simples pode ser definido como

$$
G\left(\mathbf{x}-\mathbf{x}^{\prime}\right)=\left\{\begin{array}{lll}
\frac{1}{\Delta^{3}} & \text { se } & \left|\mathbf{x}-\mathbf{x}^{\prime}\right| \leqslant \frac{\Delta}{2} \\
0 & \text { se } & \left|\mathbf{x}-\mathbf{x}^{\prime}\right|<\frac{\Delta}{2}
\end{array}\right.
$$

onde $\Delta$ é o tamanho característico do filtro, representando a frequência do corte da filtragem, mostrado na Fig. 2.4. 


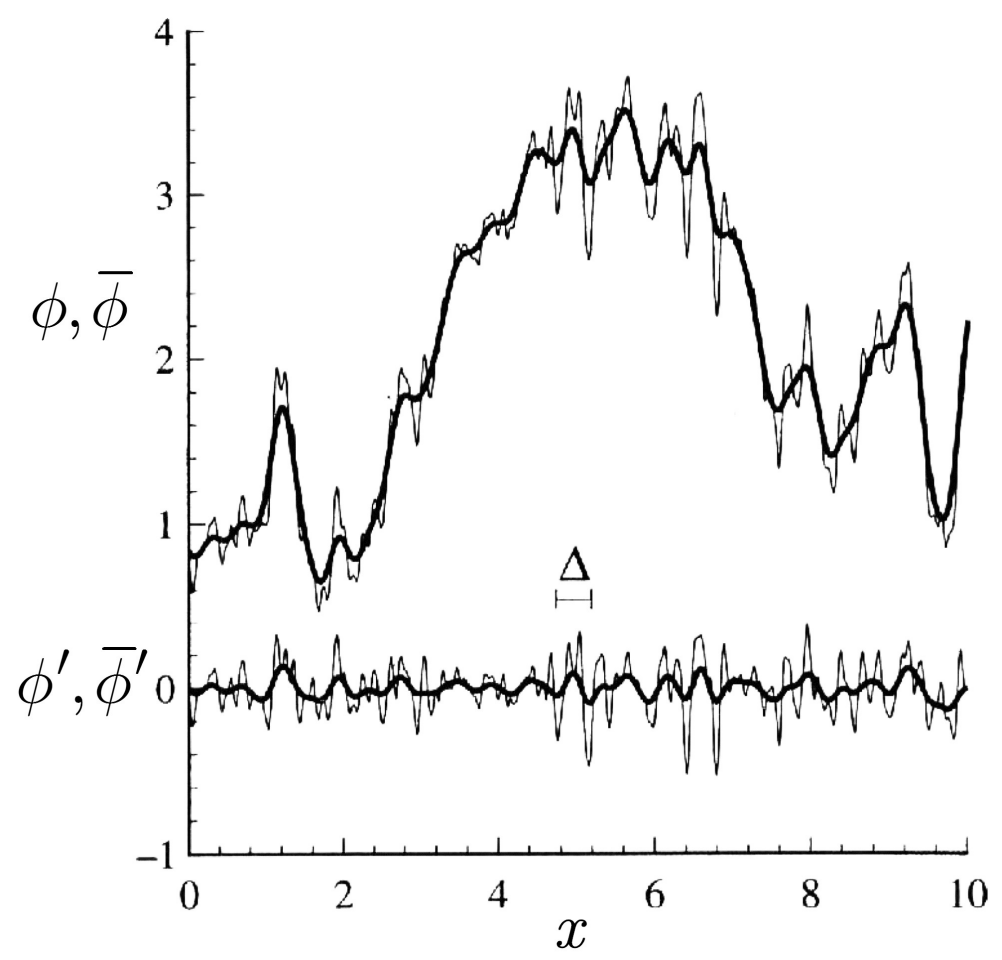

Figura 2.4: Resíduo Aplicado à Variável $\phi$ (Adaptado de (POPE, 2000)

Para a Simulação de Grandes Escalas, são descritas as seguintes propriedades da operação de filtro espacial:

$$
\bar{a}^{\prime} \neq 0, \quad \overline{\bar{a}} \overline{\bar{b}} \neq \bar{a} \bar{b}, \quad \overline{\bar{a} a^{\prime}} \neq 0, \quad \overline{\bar{a} b^{\prime}} \neq \bar{a} \bar{b}^{\prime}
$$

Aplicando a operação de filtragem dada pela equação 2.50 às Eqs. 2.8), 2.10) e 2.13, obtemos:

$$
\begin{gathered}
\nabla \cdot \overline{\mathbf{u}}=0 \\
\rho\left(\frac{\partial \overline{\mathbf{u}}}{\partial t}+\overline{\mathbf{u}} \cdot \nabla \overline{\mathbf{u}}\right)=-\nabla \bar{p}+\mu \nabla^{2} \overline{\mathbf{u}}-\rho_{0} \mathbf{g} \beta\left(\bar{T}-T_{0}\right)+\rho \mathbf{a} \\
\frac{\partial \bar{T}}{\partial t}+\overline{\mathbf{u}} \cdot \nabla \bar{T}=\alpha \nabla^{2} \bar{T}
\end{gathered}
$$

Observa-se que existe uma dificuldade na solução do sistema de equações (2.53) - 2.55), 
pois não é possível calcular os termos não-lineares $\overline{\mathbf{u} u}$ e $\overline{\mathbf{u} T}$. Para se obter solução para o sistema de equações, é necessário decompor estes utilizando a equação (2.50), dando origem aos tensores adicionais. Assim, tem-se:

$$
\overline{\mathbf{u} \mathbf{u}}=\overline{\overline{\mathbf{u}}} \overline{\mathbf{u}}+\overline{\overline{\mathbf{u}} \mathbf{u}^{\prime}}+\overline{\mathbf{u}^{\prime} \overline{\mathbf{u}}}+\overline{\mathbf{u}^{\prime} \mathbf{u}^{\prime}}
$$

Analogamente para $T$ :

$$
\overline{\mathbf{u} T}=\overline{\overline{\mathbf{u}}} \bar{T}+\overline{\overline{\mathbf{u}} T^{\prime}}+\overline{\mathbf{u}^{\prime} \bar{T}}+\overline{\mathbf{u}^{\prime} T^{\prime}}
$$

Introduzimos o tensor sub-malha (SGS - Sub Grid Stress) como

$$
\tau^{S G}=\overline{\mathbf{u} u}-\overline{\mathbf{u}} \overline{\mathbf{u}}
$$

Analogamente para a temperatura, temos o fluxo turbulento:

$$
q=\overline{\mathbf{u} T}-\overline{\mathbf{u}} \bar{T}
$$

Define-se o tensor de Leonard como:

$$
\mathbf{L}=\overline{\overline{\mathbf{u}}} \overline{\mathbf{u}}-\overline{\mathbf{u}} \overline{\mathbf{u}}
$$

Temos:

$$
\boldsymbol{\tau}^{S G}=\overline{\mathbf{u} \mathbf{u}}-\overline{\mathbf{u}} \overline{\mathbf{u}}=\underbrace{\overline{\mathbf{u} \mathbf{u}}+\overline{\overline{\mathbf{u}}} \overline{\mathbf{u}}}_{\mathbf{C}}+\underbrace{\overline{\overline{\mathbf{u}} \mathbf{u}^{\prime}}+\overline{\mathbf{u}^{\prime} \overline{\mathbf{u}}}}_{\mathbf{R}}+\underbrace{\overline{\mathbf{u}^{\prime} \mathbf{u}^{\prime}}-\overline{\mathbf{u}} \overline{\mathbf{u}}}_{\mathbf{L}}
$$

onde $\mathbf{R}$ é a tensão de Reynolds sub-malha e $\mathbf{C}$ é a tensão cruzada. 
As Eq. 2.54 e 2.55 podem ser reescritas como:

$$
\begin{gathered}
\rho\left(\frac{\partial \overline{\mathbf{u}}}{\partial t}+\overline{\mathbf{u}} \cdot \nabla \overline{\mathbf{u}}\right)= \\
=-\nabla \bar{p}+\mu \nabla^{2} \overline{\mathbf{u}}+\nabla \boldsymbol{\tau} S G-\rho_{0} \mathbf{g} \beta\left(\bar{T}-T_{0}\right)+\rho \mathbf{a}, \\
\frac{\partial \bar{T}}{\partial t}+\overline{\mathbf{u}} \cdot \nabla \bar{T}=\alpha \nabla^{2} \bar{T}+\nabla q
\end{gathered}
$$

\subsection{Modelo Sub-Malha de Smagorinski}

O modelo de Smagorinsky ((SMAGORINSKY, 1963) $)$ assume um equilíbrio entre produção, dissipação e transferência de energia nas pequenas escalas, e se utiliza de taxa de deformação para definir uma escala de comprimento de tempo, e do próprio espaçamento de malha para estabelecer uma escala de comprimento. Assim, propõe a seguinte viscosidade sub-malha, estimada a partir de grandezas resolvidas:

$$
\nu_{S G S}=\left(C_{S} \bar{\Delta}\right)^{2}|\overline{\mathbf{S}}|
$$

onde $|\overline{\mathbf{S}}|$ é o módulo do tensor taxa de deformação do campo de velocidades resolvido,

$$
|\overline{\mathbf{S}}|=\sqrt{2 \overline{\mathbf{S}}: \overline{\mathbf{S}}}
$$

$\bar{\Delta}$ é uma escala de comprimento associada com a largura do filtro espacial, e $C_{S}$ é a constante de Smagorinsky. $\mathrm{O}$ valor de $C_{S}$ pode ser obtido teoricamente através da constante de Kolmogorov $C_{k}$, que caracteriza a cascata de transferência de energia de turbulência de grandes escalas a pequenas escalas

$$
C_{S} \approx \frac{1}{\pi}\left(\frac{3 C_{k}}{2}\right)^{-3 / 4}
$$

E pode ser determinado do decaimento isotrópico da turbulência e varia entre 0,18 e 0,23 . 
Portanto esse modelo é válido para escoamentos turbulentos nos comprimentos de onda da região de equilíbrio entre produção e dissipação de energia cinética de turbulência.

\subsection{Condições de Contorno}

As condições de contorno para o presente trabalho estão divididas em dois grandes grupos: sem agitação $(\phi=0)$ e com agitação $(\phi=\phi(t))$. Como visto no início deste capítulo, $\phi$ é o ângulo de inclinação que a cavidade paralelepípeda retangular de lados $H, L$ e $W$ pode variar ao longo do tempo (Fig. 2.1). O efeito da variação $\phi$ no processo de convecção natural será estudado para fluidos Newtonianos.

\subsubsection{Condições de Contorno para $\phi=0$}

Os valores homogêneos para as variáveis $T$ e u são utilizados como condições iniciais. As condições de contorno são:

- As condições de não deslizamento para a velocidade nas paredes da cavidade, ou seja, $\mathbf{u}=0$.

- Temperatura prescrita nas paredes aquecidas: $T_{W}$

\subsubsection{Condições de Contorno para $\phi=\phi(t)$}

As condições iniciais e de parede são as mesmas utilizadas para $\phi=0$. Mas, quando impomos uma variação no ângulo de inclinção $(\phi)$ com o tempo $(\phi=\phi(t))$ novas componentes de força precisam ser incluídas na equação de quantidade de movimento (Eq. 2.9 , conforme ilustrado na figura (2.5). 


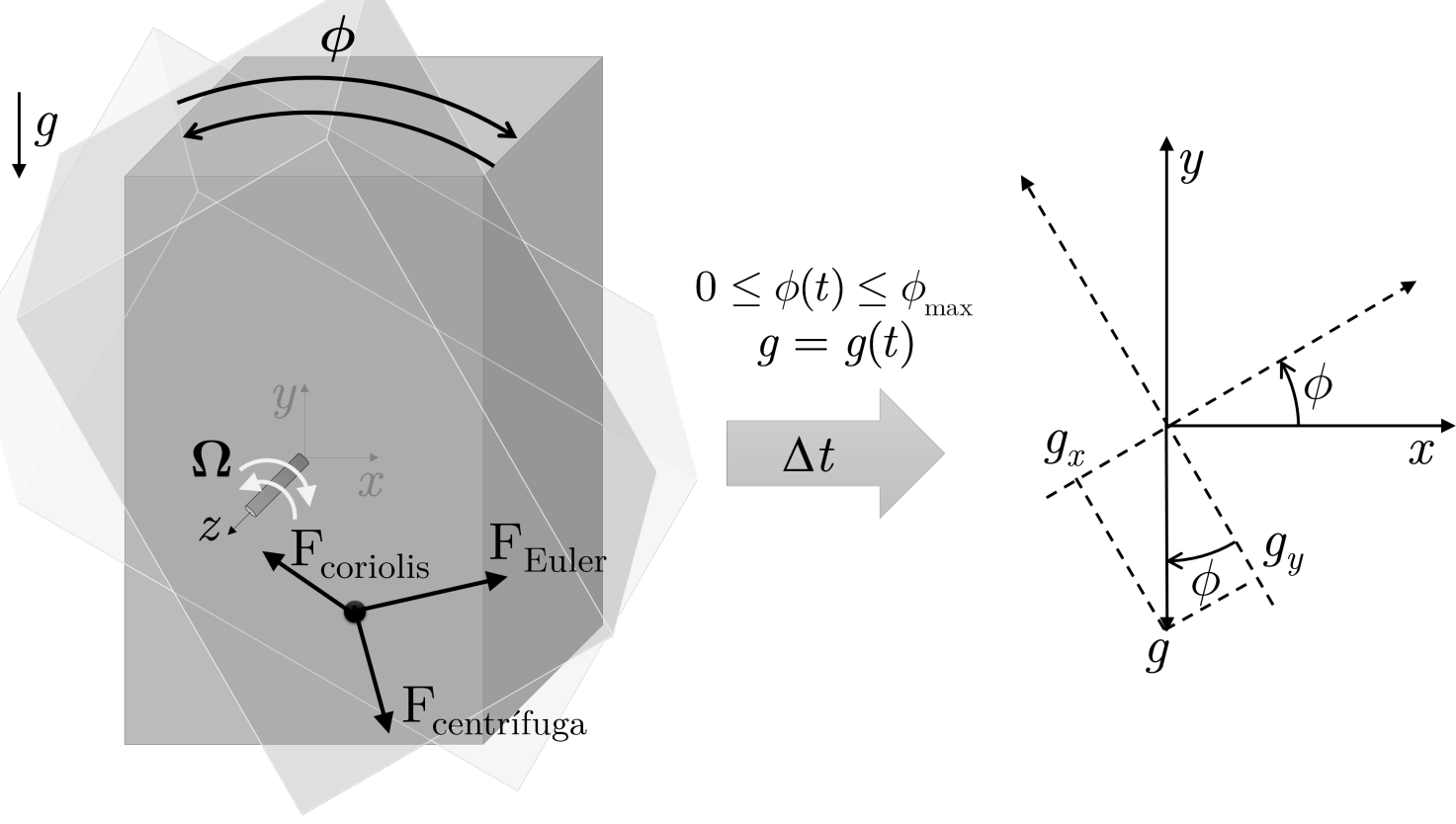

Figura 2.5: Representação da Imposição da Oscilação no Centro do Cubo.

Para isso, vamos definir primeiramente como $\phi$ pode variar de acordo com o processo de agitação e/ou rotação que são comuns na indústria de alimentos com a finalidade de agilizar o processo de conveç̧ão natural em cavidades ((GUMERATO, 2004)). Abaixo temos a equação de $\phi$ com esses dois processos:

$$
\phi(t)=\underbrace{\left|\operatorname{sen}\left(\omega_{\text {agit }} t\right)\right| \phi_{\max }}_{\text {agitação }}+\underbrace{\omega_{\text {rot }} t}_{\text {rotação }}
$$

em que $\omega$ é a frequência angular relacionada ao processo de agitação, $\omega_{\text {agit }}=2 \pi f_{\text {agit }}$, ou ao processo de rotação, $\omega_{\text {rot }}=2 \pi f_{\text {rot }}$. Sendo $f$ a frequência normal e $\phi_{\max }$ o ângulo máximo de varição da inclinação da cavidade. Dependendo do problema a ser simulado, o domínio pode passar por um processo de agitação ou rotação "pura", bastando definir $f_{\text {agit }}$ ou $f_{\text {rot }}$ como nulos, respectivamente.

Ao impormos o processo de agitação e/ou rotação alteramos, principalmente, o vetor gravidade, g, que passará a variar de acordo com $\phi=\phi(t)$ e terá as seguintes componentes: 


$$
g(t)=g_{0} \operatorname{sen} \phi(t) \mathbf{i}-g_{0} \cos \phi(t) \mathbf{j}
$$

sendo $g_{0}$ é o valor escalar da gravidade.

Na sequência, definimos a velocidade angular, $\Omega$, obtida pela derivada de $\phi$ em função do tempo $(d \phi / d t)$, ou seja:

$$
\Omega(t)=\left|\omega_{\text {agit }} \cos \left(\omega_{\text {agit }} t\right)\right| \phi_{\max }+\omega_{\text {rot }}
$$

Consequentemente, serão introduzidas as seguintes forças: Centrífuga, Coriolis e Euler, respectivamente:

$$
\begin{aligned}
\mathbf{F}_{\text {centrífuga }} & =\rho[\Omega \times(\Omega \times \mathbf{r})] \\
\mathbf{F}_{\text {coriolis }} & =\rho(2 \Omega \times \mathbf{u}) \\
\mathbf{F}_{\text {Euler }} & =\rho \frac{d \Omega}{d t} \times \mathbf{r}
\end{aligned}
$$

Substituindo essas forças na equação de quantidade de movimento (Eq. (2.9)), temos:

$$
\rho\left(\frac{\partial \mathbf{u}}{\partial t}+\mathbf{u} \cdot \nabla \mathbf{u}\right)=\nabla \boldsymbol{\tau}-\rho \mathbf{g} \beta\left(T-T_{0}\right)+\Omega \times(\Omega \times \mathbf{r})+2 \Omega \times \mathbf{u}+\frac{d \Omega}{d t} \times \mathbf{r}
$$

\subsection{Adimensionalizações}

O OpenFOAM trabalha com as equações e os formatos de arquivos de entrada e saída (tais como, escalares, vetores, tensores, listas e campos) na forma dimensional pelo Sistema Internacional de medidas (SI), portanto, não havendo a necessidade da adi- 
mensionalização. Porém, com a finalidade de comparação de resultados com dados da literatura, o processo de adimensionalizadas ocorre na etapa de pós-processamento.

\subsubsection{Números Adimensionais Importantes para Convecção Natural}

As variáveis apresentadas, principalmente, nos resultados foram adimensionalizadas usando a escala $H$ para o comprimento, $(\alpha / H) \mathrm{Ra}^{0.5}$ para a velocidade e $\left(H^{2} / \alpha\right) \mathrm{Ra}^{-0.5}$ para o tempo, mostradas abaixo:

$$
\mathbf{x}^{*}=\frac{\mathbf{x}}{H}, \quad \mathbf{u}^{*}=\frac{\mathbf{u}}{U_{0}}, \quad t^{*}=\frac{U_{0} t}{H},
$$

em que Ra é o número de Rayleygh global, dado por

$$
\mathrm{Ra}=\frac{g \beta\left(T_{w}-T_{0}\right) H^{3}}{\nu \alpha}
$$

Semelhante ao que é apresentado em (FAGHRI; ZHANG; HOWELL, 2010), a velocidade de referência $U_{0}$ foi definida em função de Ra, ou seja,

$$
U_{0}=\frac{\alpha}{H} \sqrt{\mathrm{Ra}}=\sqrt{\frac{g \beta\left(T_{w}-T_{0}\right) H \alpha}{\nu}}
$$

Com essa escolha para velocidade de referência, podemos escrever o número de Reynolds (Re):

$$
\operatorname{Re}=\sqrt{\frac{\mathrm{Gr}}{\mathrm{Pr}}}=\frac{\sqrt{\mathrm{Ra}}}{\operatorname{Pr}}
$$

onde $\mathrm{Gr}=g \beta\left(T_{w}-T_{0}\right) H^{3} / \nu^{2}$ é o número de Grashof, $\operatorname{Pr}=\nu / \alpha$ é o número de Prandtl e $\mathrm{Ra}=\mathrm{Gr} \cdot \operatorname{Pr}$. 
A temperatura adimensional, $\theta$, é definida por

$$
\theta=\frac{T-\bar{T}}{T_{w}-T_{0}}
$$

em que o valor de $\bar{T}$ é dado por $\bar{T}=\frac{1}{V} \iiint_{V} T d V$ e calculado para cada instante $t$ observado da simulação.

O número de Nusselt médio $(\overline{\mathrm{Nu}})$ pode ser obtido a partir do fluxo de calor das paredes aquecidas como ((BEJAN, 2004) $)$ :

$$
\overline{\mathrm{Nu}}=\frac{\bar{h} H}{k},
$$

considerando

$$
\bar{h}=\frac{\overline{q^{\prime \prime}}}{\left(T_{w}-\bar{T}\right)},
$$

o coeficiente de transferência de calor e

$$
\overline{q^{\prime \prime}}=\frac{k}{A_{\Gamma}} \iint_{\Gamma} \frac{\partial T}{\partial \eta} d \Gamma
$$

o fluxo de calor na parede representada por $\Gamma$, com área $A_{\Gamma}$ na direção $\eta$, perpendicular à parede correspondente.

A energia cinética do campo de escoamento $(\mathrm{K})$, adimensionalizada por $(1 / 2) \rho \operatorname{Ra}(\alpha / H)^{2}$, é calculado pela integral sobre o volume $(V)$ da cavidade:

$$
\mathrm{K}=\frac{1}{2} \rho \iint_{V}(\mathbf{u} \cdot \mathbf{u}) d V
$$


Ao longo da apresentação dos resultados, o número de Rayleygh poderá ser avaliado localmente, $\mathrm{Ra}^{*}$ e, nesse caso, é baseado na temperatura média, $\bar{T}$, ao invés da temperatura de referência inicial, ou seja

$$
\mathrm{Ra}^{*}=\frac{g \beta\left(T_{w}-\bar{T}\right) H^{3}}{\nu \alpha}
$$

Da mesma forma análoga, o número de Nusselt local $(\mathrm{Nu})$ é calculado pontualmente sobre a face da geometria da cavidade, conforme a equação abaixo:

$$
\mathrm{Nu}=\frac{h H}{k}, \quad h=\frac{q^{\prime \prime}}{\left(T_{w}-\bar{T}\right)}, \quad q^{\prime \prime}=k \frac{\partial T}{\partial \eta} .
$$

Para a situação em que $\phi=\phi(t)$ o número de Rayleigh será diferenciado, sendo denotado por $\operatorname{Ra}_{\omega}$

$$
\operatorname{Ra}_{\omega}=\frac{\Omega \beta\left(T_{w}-T_{0}\right) H^{4}}{\nu \alpha}
$$

em que $\Omega$ é a magnitude da velocidade angular. 


\section{Capítulo 3}

\section{Metodologia Numérica}

Apesar de muitos problemas de transferência de calor serem observados em geometrias simples, conforme apresentados na Fig. (2.5), a solução ocorre pela resolução simultânea de um sistema de equações diferenciais parciais não-lineares e acopladas. Devido à complexidade de se obter soluções analíticas para tais problemas, torna-se necessária a utilização de métodos numéricos para a obtenção dos campos de escoamento e térmico do domínio estudado ((MALISKA, 2004)). Para resolver o sistema de equações transientes e não-linear dado pelas Equações (2.8), 2.10) e (2.13), primeiramente se fez a discretização das equações no espaço e tempo através do Método de Volumes Finitos com o OpenFOAM.

\subsection{Discretização pelo Método de Volumes Finitos}

O método numérico utilizado na discretização das equações no OpenFOAM toma como base o Método de Volumes Finitos (MVF) ((PATANKAR, 1980), (MALISKA, 2004), (JASAK, 1996), (VERSTEEG; MALALASEKERA, 1995) e (SILVA, 2008)). O termo "discretização" se refere à aproximação de um problema contínuo, o qual é representado por equações diferenciais parciais, em quantidades discretas dos domínios do espaço, do tempo e das equações de transporte. A discretização no domínio do espaço divide o domínio físico em um conjunto de subdomínios, ou seja, de volumes de controle, conhecidos como células, os quais estão conectados entre si dividindo uma mesma face 
ou conectados ao limite do domino, ou seja, ao seu contorno e desta forma criam o que se chama de malha computacional. As células são contíguas, ou seja, elas não coincidem umas com as outras e preenchem completamente o domínio de cálculo ((SILVA, 2008)).

A discretização do domínio temporal é utilizada em problemas transientes e pode ser obtida dividindo o espaço de tempo em um conjunto de intervalos, ou passos de tempo $\Delta t$ (SILVA, 2008). O intervalo de tempo pode mudar durante a solução numérica, manter-se em um valor pré especificado ou até mesmo ser calculado durante a simulação através de critérios de convergência pré estabelecidos, como por exemplo utilizar o critério de convergência baseado no número de Courant-Friedrich-Levy ou simplesmente número de Courant apresentado por (COURANT; FRIEDRICHS; LEWY, 1967).

A Figura 3.1 apresenta a discretização no domínio de cálculo, o qual é constituído pelos domínios temporal e espacial discretizados.
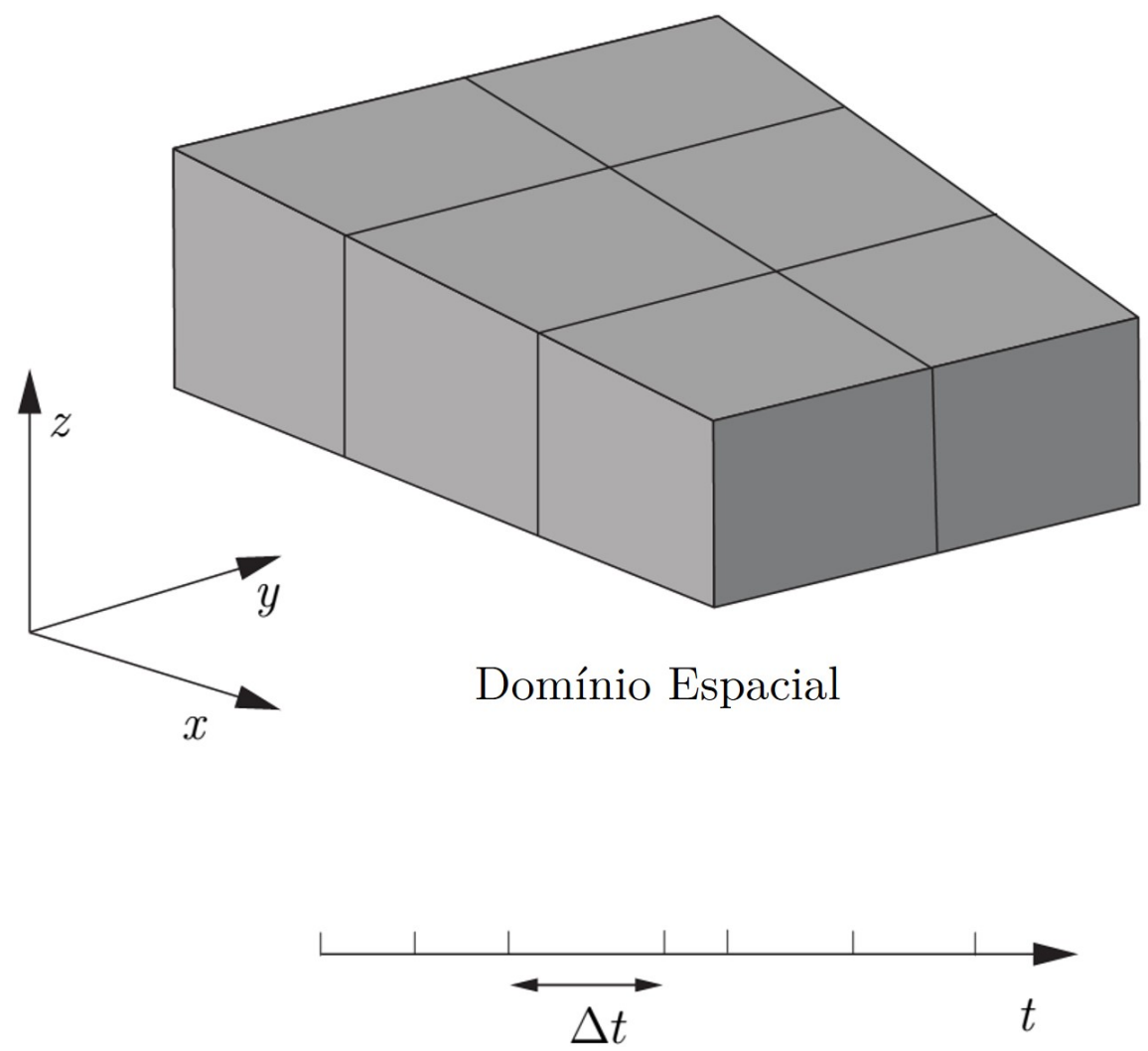

Domínio Temporal

Figura 3.1: Discretização do Domínio da Solução (Adaptado de (OPENCFD, 2014a)) 


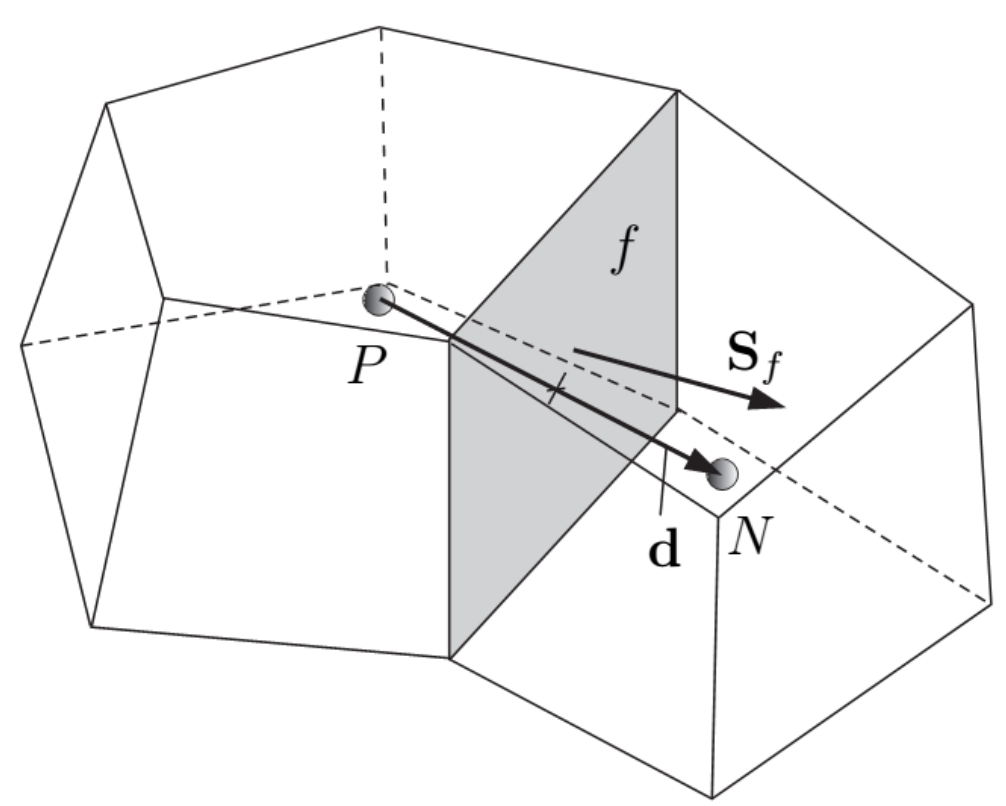

Figura 3.2: Parâmetros na Discretização do Método de Volumes Finitos (Adaptado de (OPENCFD, 2014a))

Juntamente com as discretizações do domínio espacial realiza-se a discretização das equações de transporte que governam as leis físicas do problema, gerando um sistema de equações algébricas em termos de quantidades discretas definidos em locais específicos no domínio do espaço.

Na Figura 3.2 são mostradas duas células típicas. Uma das células é delimitada por um conjunto de faces, dando o rótulo genérico $f$. As faces da célula são divididas em dois grupos: faces internas (entre duas células) e faces do contorno, que coincidem com os limites do domínio espacial. O vetor posição no centro de uma face plana (em que todos os vértices se encontram no mesmo plano) $\mathbf{x}_{f}$ é definido pela equação:

$$
\int_{S}\left(\mathbf{x}-\mathbf{x}_{f}\right) d S=0
$$

No Método de Volumes Finitos as variáveis dependentes e outras propriedades são armazenadas no centro $P$ da célula, conforme pode ser observado na Fig. (3.2).

Na Figura 3.2 é possível definir um vetor posição do centro da célula $\mathbf{x}_{P}$, tal como, 


$$
\int_{V_{P}}\left(\mathbf{x}-\mathbf{x}_{P}\right) d V=0
$$

Conforme SILVA (2008), pode-se também definir o vetor $\mathbf{S}$ como sendo um vetor normal à face e sua magnitude é igual à área da face. O sentido deste vetor é da célula de interesse $P$ para a célula vizinha $N$. O vetor unitário normal à face $n$ é definido de acordo como sendo $\mathbf{n}=\frac{\mathbf{S}}{|\mathbf{S}|}$ e na Fig. 3.2 tem-se o vetor $\mathbf{d}$ cujo sentido é do centro da célula de interesse $P$ ao centro da célula vizinha $N$, isto é, $\mathbf{d}=\mathbf{x}_{N}-\mathbf{x}_{P}$. Pode-se dizer que uma malha computacional é ortogonal quando o vetor $\mathbf{d}$ é ortogonal à face plana, ou seja, paralelo ao vetor $\mathbf{S}$, para cada face na malha (WELLER et al., 1998) e OPENCFD, 2014b).

Uma questão delicada sobre a discretização diz respeito ao armazenamento do campo de variáveis na malha. Normalmente, todas as variáveis dependentes (efetivamente solucionadas) são alocadas no centro dos volumes. Este tipo de alocação, denominado arranjo co-localizado, se trona a escolha mais óbvia pela sua simplicidade de controle dos índices da malha na implementação computacional. Porém, podem aparecer problemas no arranjo co-localizado, como oscilação e avaliação do gradiente de pressão (MALISKA, 2004). Contudo, com os avanços das técnicas numéricas ((PERIć; KESSLER; SCHEUERER, 1988) e (RHIE; CHOW, 1983)) estes problemas foram resolvidos e, desde então, o arranjo co-localizado é usado na maioria dos códigos CFD, inclusive no OpenFOAM.

O processo de discretização utilizado pelo MVF transforma as equações diferenciais de variáveis continuas em um sistema de equações algébricas correspondente. Partindo de uma equação de transporte para uma variável $\varphi$ tensorial genérica

$$
\frac{\partial(\rho \varphi)}{\partial t}+\nabla \cdot(\rho \mathbf{u} \varphi)=\nabla \cdot(\Gamma \nabla \varphi)+S_{\varphi}(\varphi)
$$

onde $\rho$ é a massa específica, u é o campo de velocidade, $\Gamma$ é o coeficiente de difusão e $S_{\varphi}(\varphi)$ é termo fonte, sua discretização por volumes finitos é obtida integrando-a sobre um volume de controle $V_{P}$ e em um intervalo de tempo. 


$$
\begin{gathered}
\int_{t}^{t+\Delta t}\left[\int_{V_{P}} \frac{\partial(\rho \varphi)}{\partial t} d V+\int_{V_{P}} \nabla \cdot(\rho \mathbf{u} \varphi) d V\right] d t= \\
\int_{t}^{t+\Delta t}\left[\int_{V_{P}} \nabla \cdot(\Gamma \nabla \varphi) d V+\int_{V_{P}} S_{\varphi}(\varphi) d V\right] d t
\end{gathered}
$$

O teorema de Gauss é usado para converter as integrais de volume dos termos derivativos no espaço em integrais sobre a superfície $S$ no contorno dos volumes de controle.

$$
\int_{V}(\nabla \cdot \varphi) d V=\int_{\partial V} d \mathbf{S} \cdot \varphi
$$

O teorema de Gauss é utilizado na Eq. 3.4 para aproximar seus termos pelo somatório dos fluxos das propriedades pelas faces do volume de controle. Este procedimento é detalhado na sequência.

O termo convectivo $\nabla \cdot(\rho \mathbf{u} \varphi)$ da Eq. 3.4 é aproximado convertendo as integrais em volume em superfície usando o teorema de Gauss e somando-as em todo o volume de controle:

$$
\begin{aligned}
\int_{V} \nabla \cdot(\rho \mathbf{u} \varphi) d V=\int_{\partial V} d \mathbf{S} \cdot(\rho \mathbf{u} \varphi) & \approx \sum_{f} \mathbf{S} \cdot(\rho \mathbf{u})_{f} \varphi_{f(F, M, \gamma)} \\
& =\sum_{f} F \varphi_{f(F, M, \gamma)},
\end{aligned}
$$

onde $F$ é o fluxo mássico através da face $f$ definido como $F=\mathbf{S} \cdot(\rho \mathbf{u})_{f}$. O valor da propriedade na face $\varphi_{f(F, M, \gamma)}$ pode ser obtido usando uma função de interpolação $M$ pré-definida. Esta última usualmente utiliza informações do próprio volume de controle e seus vizinhos, além de requerer o fluxo $F$ sobre a face $f$ e um ou mais parâmetros $\gamma$ para efetuar a interpolação. Existem vários métodos de interpolação, entre os quais pode-se citar as abordagens de interpolação linear (diferenças centrais), upwind, QUICK, MUSCL, TVD (Total Variation Diminishing) e NVD (Normalised Variable Diagram). A formulação destes métodos encontram-se em vários trabalhos ((MALISKA, 2004), (JASAK, 1996), (VERSTEEG; MALALASEKERA, 1995) e (TORO, 
2009)) e suas formulações só serão abordadas quando for necessário.

Da mesma forma, o termo difusivo $\nabla \cdot(\Gamma \nabla \varphi)$ da Eq. 3.4 é aproximado usando o teorema de Gauss:

$$
\int_{V} \nabla \cdot(\Gamma \nabla \varphi) d V=\int_{\partial V} d \mathbf{S}(\Gamma \nabla \varphi) \approx \sum_{f} \Gamma_{f}\left(\mathbf{S} \cdot \nabla_{f} \varphi\right)
$$

considerando $\Gamma$ como uma variável escalar. Em malhas ortogonais, o gradiente $\nabla_{f} \varphi$ pode ser obtido pela expressão

$$
\nabla_{f} \varphi=\frac{\varphi_{N}-\varphi_{P}}{|\mathbf{d}|}
$$

Usando a Eq. 3.8, o gradiente de $\varphi$ avaliado na face é calculado usando os dois valores centrais localizados entre a face $f$. Como alternativa, a discretização do gradiente pode ser calculada usando o teorema de Gauss para a integral no volume:

$$
\int_{V} \nabla \varphi d V=\int_{\partial S} d \mathbf{S} \varphi \approx \sum_{f} \mathbf{S} \varphi_{f}
$$

onde o valor de $\varphi_{f}$ é obtido através de uma função de interpolação.

Termos fontes, como $S_{\varphi}(\varphi)$ mostrado na Eq. 3.4. podem ser funções genéricas de $\varphi$. Assim, estes termos são linearizados antes da discretização:

$$
S_{\varphi}(\varphi)=S_{I} \varphi+S_{E},
$$

sendo que $S_{I}$ e $S_{E}$ podem ser dependentes de $\varphi$. Este termo integrado no volume de controle resulta em:

$$
\int_{V} S_{\varphi}(\varphi) d V=S_{I} \varphi_{P} V_{P}+S_{E} V_{P}
$$


Ao assumir que os volumes de controle não variam com o tempo, a Eq. 3.4 pode ser reescrita usando as Eqs. 3.6, 3.7 e 3.11.

$$
\begin{aligned}
& \int_{t}^{t+\Delta t}\left[\left(\frac{\partial(\rho \varphi)}{\partial t}\right)_{P} V_{P}+\sum_{f} F \varphi_{f(F, M, \gamma)}\right] d t= \\
& \int_{t}^{t+\Delta t}\left[\sum_{f} \Gamma_{f}\left(\mathbf{S} \cdot \nabla_{f} \varphi\right)+\left(S_{I} \varphi_{P}+S_{E}\right) V_{P}\right] d t
\end{aligned}
$$

A expressão acima usualmente é dita como a forma semi-discretizada da equação de transporte (JASAK, 1996).

Assumindo uma variação linear de $\varphi(t)$ no tempo (JASAK, 1996), a discretização da derivada no tempo mostrada na Eq. 3.12 e a integral no tempo podem ser calculadas diretamente como

$$
\begin{gathered}
\left(\frac{\partial(\rho \varphi)}{\partial t}\right)_{P}=\frac{\rho_{P}^{n} \varphi_{P}^{n}-\rho_{P}^{0} \varphi_{P}^{0}}{\Delta t}, \\
\int_{t}^{t+\Delta t} \varphi(t) d t=\frac{1}{2}\left(\varphi^{0}+\varphi^{n}\right) \Delta t,
\end{gathered}
$$

onde $\varphi^{n}=\varphi(t+\Delta t)$ representa o novo valor de $\varphi$ para passo de tempo a ser resolvido enquanto $\varphi^{0}=\varphi(t)$ é o valor no passo anterior. Utilizando as Eqs. $3.12,3.13$ e 3.14. obtém-se a forma discretizada, temporal e espacial, da Eq. 3.4 .

$$
\begin{aligned}
\rho_{P} \frac{\varphi_{P}^{n}-\varphi_{P}^{0}}{\Delta t} V_{P} & +\frac{1}{2} \sum_{f} F^{n} \varphi_{f(F, M, \gamma)}^{n}-\frac{1}{2} \sum_{f} \Gamma_{f}^{n} \mathbf{S} \cdot\left(\nabla_{f} \varphi\right)^{n} \\
& +\frac{1}{2} \sum_{f} F^{0} \varphi_{f(F, M, \gamma)}^{0}-\frac{1}{2} \sum_{f} \Gamma_{f}^{0} \mathbf{S} \cdot\left(\nabla_{f} \varphi\right)^{0} \\
& +\frac{1}{2}\left(S_{E}^{n}+S_{E}^{0}\right) V_{P}+\frac{1}{2}\left(S_{I}^{n} \varphi_{P}^{n}+S_{I}^{0} \varphi_{P}^{0}\right) V_{P}
\end{aligned}
$$


A formulação da discretização temporal apresentada na Eq. 3.15é o método de segunda ordem de Crank-Nicholson. Este método requer os valores de $\varphi$ e $\nabla \varphi$ no volume de controle e nas suas faces no instante de tempo a ser resolvido e passado. Os valores nas faces são calculados a partir dos valores no centro dos volumes em cada lado da face e usando métodos adequados para interpolação dos termos convectivos e difusivos.

\subsubsection{Sistema Algébrico de Equações}

De fato, o objetivo é obter o novo valor de $\varphi_{P}$. Como $\varphi_{f}$ e $\nabla_{f} \varphi$ também dependem do valor de $\varphi$ nos volumes vizinhos, a Eq. 3.15 pode ser representada como uma equação algébrica válida para o volume $P$ :

$$
a_{P} \varphi_{P}^{n}+\sum_{N} a_{N} \varphi_{N}^{n}=b_{P}
$$

Ao considerar todos os volumes de controle da malha, a Eq. 3.16 pode ser escrita como um sistema algébrico de equações,

$$
\mathcal{A} \varphi=\mathbf{b}
$$

onde $\mathcal{A}$ é uma matriz esparsa com coeficientes diagonais $a_{P}$ e não diagonais $a_{N}$, enquanto que $\varphi$ e b são, respectivamente, os vetores das variáveis $\varphi$ e os termos fontes referentes ao centro dos volumes. A matriz $\mathcal{A}$ pode ser decomposta em duas matrizes contendo apenas os coeficientes diagonais $\mathcal{D}$ e os não-diagonais $\mathcal{N}$, tal que:

$$
\mathcal{A}=\mathcal{D}+\mathcal{N}
$$

Portanto, a estrutura da matriz de coeficientes $\mathcal{A}$ pode variar dependendo da dimensão do problema, da forma de discretização das equações e da ordenação dos volumes de controle na malha. A estrutura da matriz é um parâmetro importante na escolha do método mais apropriado para resolver o sistema linear. Em aplicações CFD, cerca 
de $80 \%$ do tempo de computação necessário para resolver um dado problema está associado à solução de sistema linear. Os outros $20 \%$ estão associados à obtenção das equações discretizadas (SILVA, 2008).

Os métodos numéricos para solução de sistemas lineares podem ser divididos em duas classes: diretos e iterativos. Os métodos diretos determinam a solução exata, a menos de erros de truncamento, em um número finito de operações matriciais. Eles são indicados para sistemas lineares com um número não muito grande de equações (até algumas centenas) ou com uma matriz dos sistema cuja estrutura permita um método especial de solução. A limitação destes métodos a sistemas pequenos ocorre por dois motivos. Primeiro, o número de operações (matemáticas) cresce muito rapidamente com o tamanho do sistema, o que aumenta o custo computacional superlinearmente. Além disso, o alto número de operações leva ao aumento dos erros de truncamento, o que degrada a precisão da solução.

Os métodos iterativos foram desenvolvidos para superar as deficiências dos métodos diretos no tratamento de grandes sistemas. Os métodos iterativos também ganharam espaço devido a sua facilidade de implementação em computadores de alto desempenho com memória compartilhado ou distribuída (supercomputadores e cluster), quando comparado aos métodos diretos. Alguns dos métodos iterativos, como o Gauss-Seidel, o Gradiente Conjugado (CG) e suas variantes e o Algebric Multigrid (AMG) podem ser citados (SAAD, 2003) e estão implementados no OpenFOAM.

\subsubsection{Notação da Formulação Discreta}

Conforme SILVA (2008), o processo de discretização pode ser efetuado de forma implícita ou explícita, onde a primeira é utilizada para construir os sistema linear através da discretização. Já a segunda forma realiza os cálculos da operação de forma explícita, usando os valores de $\varphi$ no instante atual. (RUSCHE, 2003 apud WELLER, 2002 ) introduziu uma notação específica para volumes finitos que permite especificar o tratamento aplicado no processo de discretização.

Com isso, a discretização de um operador $\mathcal{L}$ é representada por $\lfloor\mathcal{L}[\varphi]\rfloor$. O operador $\mathcal{L}$ pode ser derivativo no espaço ou no tempo, ou mesmo um termo fonte que possui tratamento implícito. A variável dependente $\varphi$ colocada entre colchetes deve ter tra- 
tamento implícito e ser idêntica em todos os termos da equação. Por outro lado, os termos discretizados de forma explícita não são representados entre colchetes. Assim, as operações implícitas e explícitas podem ser diferenciadas usando esta notação. Um resumo é apresentado na Tabela 3.1 .

Tabela 3.1: Notação da Discretização por Volumes Finitos

\begin{tabular}{lcc}
\hline Termo & Notação por Volumes Finitos & Termo Discretizado \\
\hline Derivada no Tempo & $\left\lfloor\frac{\partial \rho[\varphi]}{\partial t}\right\rfloor$ & $\frac{\rho_{P}^{n} \varphi_{P}^{n}-\rho_{P}^{0} \varphi_{P}^{0}}{\Delta t} V_{P}$ \\
Convectivo & $\sum_{f} F \varphi_{f}(F, M, \gamma)$ \\
Difusivo & $\left.\left\lfloor\nabla \cdot\left(\Gamma \nabla[\varphi]_{f}(F, M, \gamma)\right\rfloor\right]\right)$ & $\sum_{f} \Gamma_{f} \mathbf{S} \cdot\left(\nabla_{f} \varphi\right)$ \\
Divergente & $\sum_{f} \mathbf{S} \cdot \varphi_{f}^{0}$ \\
Gradiente no Volume & $\nabla \varphi$ & $\sum_{f} \mathbf{S} \varphi_{f}^{0}$ \\
Fonte Implícito & $\left\lfloor S_{I}[\varphi]\right\rfloor$ & $S_{I} V_{P} \varphi^{n}$ \\
Fonte Explícito & $S_{E}$ & $S_{E} V_{P}$ \\
\hline
\end{tabular}

Seguindo a notação mostrada na Tabela 3.1. o sistema linear obtido pela discretização da equação de transporte (Eq. 3.3) pode ser escrita como colocado abaixo:

$$
\mathcal{A}:=\left\lfloor\frac{\partial \rho[\varphi]}{\partial t}\right\rfloor+\left\lfloor\nabla \cdot\left(F[\varphi]_{f(F, M, \gamma)}\right)\right\rfloor-\lfloor\nabla \cdot(\Gamma \nabla[\varphi])\rfloor-\left\lfloor S_{I}[\varphi]\right\rfloor-S_{E}
$$

Note que a equação discretizada para cada volume de controle é idêntica àquela mostrada na Eq. 3.12

Uma vez que o sistema linear é montado, pode-se definir operadores especiais para extrair os coeficientes da matriz e seus termos fonte usando, respectivamente, $\mathcal{A}_{\mathcal{A}} \equiv \mathcal{A}$ e $\mathcal{A}_{S} \equiv \mathbf{b}$. Da mesma forma, matrizes contendo os coeficientes diagonais e não diagonais também podem ser obtidos a partir de $\mathcal{A}_{D} \equiv \mathcal{D}$ e $\mathcal{A}_{N} \equiv \mathcal{N}$, respectivamente.

O operador "H", $\mathcal{A}_{H}$, definido na sequência, é usado exaustivamente nos algoritmos numéricos do OpenFOAM. Sua definição parte do método iterativo de Jacobi para 
obtenção de uma solução aproximada das equações discretizadas como mostrado na Eq. 3.20 .

$$
\varphi \approx \mathcal{A}_{D}^{-1} \mathcal{A}_{H}
$$

Logo, o operador "H" pode ser obtido utilizando as Eqs. 3.18 e 3.20 .

$$
\mathcal{A}_{H}=\mathcal{A}_{S}-\mathcal{A}_{N} \varphi
$$

\subsection{Linguagem de Programação no OpenFOAM}

A técnica de Orientação a Objetos em $\mathrm{C}++$ usada pelo OpenFOAM permitiu criar tipos de dados muito próximos aos usados em mecânica do contínuo. A técnica de sobrecarga de operadores 1 permitiu que a simbologia matemática usual fosse aplicada para operações básicas. Assim, as equações de mecânica do contínuo apresentadas como equações diferenciais parciais e os conceitos escalares, vetores, tensores e seus respectivos campos, assim como a álgebra tensorial e sistemas de unidades podem ser invocados no OpenFOAM usando uma sintaxe parecida com a notação matemática usual (SILVA, 2008).

As classes implementadas no OpenFOAM declaram tipos e operações associadas que fazem parte da linguagem matemática utilizada na engenharia e no meio científico. $\mathrm{O}$ campo de velocidades pode ser apresentado no código de programação pelo símbolo U e a magnitude do campo de velocidade poder mag (U). A velocidade é um campo vetorial e, portanto, deve existir, em um código de orientação a objetos, uma classe vectorField. Então, o campo de velocidade pode ser visto como um objeto da classe vectorField.

\footnotetext{
${ }^{1}$ Permite transformar uma listagem de programa complexa em outra mais intuitiva. A sobrecarga, simplificadamente, consiste na redefinição de itens já existentes. Em outras palavras, ela permite que sejam definidas duas ou mais funções com o mesmo nome, desde que suas listas de argumentos sejam suficientemente diferentes para as chamadas a serem resolvidas. Nesta situação, quando diversas declarações de funções diferentes são especificadas por um único nome no mesmo escopo, diz-se que as funções que compartilham o mesmo nome estão sobrecarregadas (DEITEL; DEITEL, 2001) e (YANG, 2001).
} 
A estrutura das classes restringe o desenvolvimento do código dentro das próprias classes, torando o código mais fácil de manipular. Novas classes podem herdar propriedades de outras classes, por exemplo, um vectorField pode ser derivado de uma classe vector e uma classe Field. O $\mathrm{C}++$ fornece um mecanismo chamado de classes template, de forma que a classe Field $<$ Type $>$ pode representar um campo de qualquer Type, como scalar, vector e tensor. As características gerais da classe template são passadas para qualquer classe criada a partir deste template. Os templates e a herança reduzem a duplicação do código e criam hierarquias de classe que impõe uma estrutura ao código.

Assim, utilizando as classes do OpenFOAM, a sintaxe de escrita dos solvers se assemelha à solução de equações diferenciais parciais. Por exemplo, a Eq. 3.22

$$
\frac{\partial \rho \mathbf{u}}{\partial t}+\nabla \cdot(\Phi \mathbf{u})-\nabla^{2}(\mu \mathbf{u})=-\nabla p
$$

é representada pelo Cód. 3.1 em $\mathrm{C}++$

Código 3.1: Representação da Eq. 3.22 pelo OpenFOAM

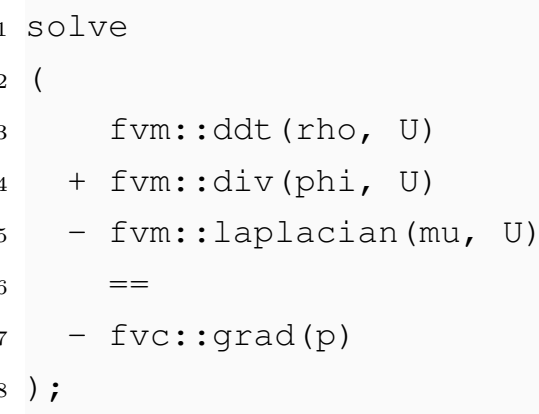

onde os templates fvm e fvc referem-se, respectivamente, a operações de discretização implícita e explícita usando volumes finitos. O sistema linear resultante é resolvido com a função de classe solve. 


\subsection{Implementação do Código CFD no OpenFOAM}

Como mencionado anteriormente, as classes e templates ${ }^{2}$ podem encapsular tipos e operações sobre variáveis. As classes devem ser declaradas no início do código através de bibliotecas para que seja possível usar e acessar comandos presentes na mesma. A principal biblioteca do OpenFOAM está declarada em fVCFD.H, pois, agrega várias outras bibliotecas que são importantes para o pleno funcionamento do código (ver Fig. 3.3. . Em conjunto, essas bibliotecas são capazes de alocar, manipular e operar variáveis de campo interpretadas pelo OpenFOAM.

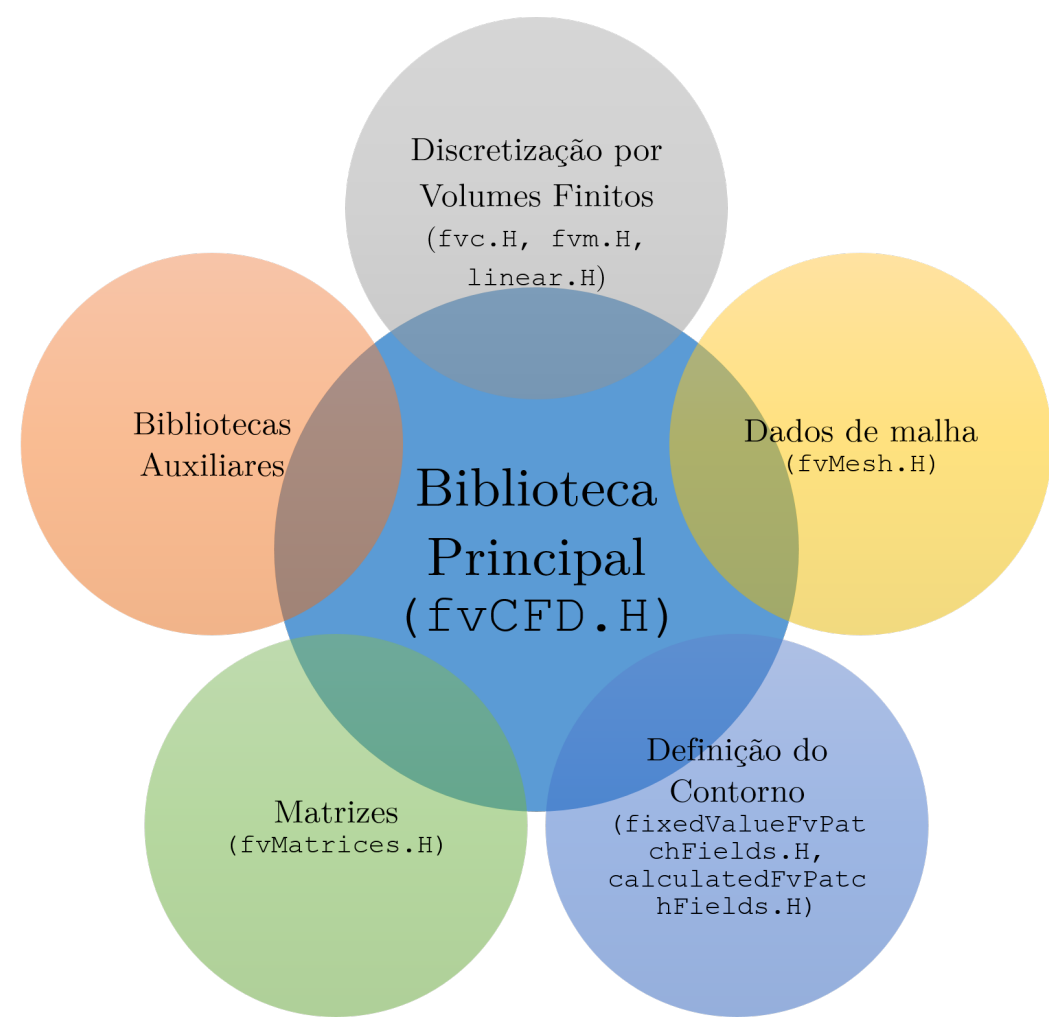

Figura 3.3: Bibliotecas do OpenFOAM (Adaptado de (SILVA, 2008))

Na execução de um código CFD no OpenFOAM, primeiramente a geometria e a malha computacional são alocados usando a classe poluMesh, que armazena informações topológicas e geométricas da malha. Estes dados podem ser atualizados durante a

\footnotetext{
${ }^{2} \mathrm{~A}$ partir desse momento, para qualquer menção às classes deve-se incluir os templates implicitamente
} 
simulação, tendo liberdade para apagar informações sobre os volumes de controle (volume, área das faces, posição do centro do volume/face, etc.) e recalculá-las quando for necessário. Assim, é possível apagar ou modificar dados referentes a mudanças tológicas (refinamento de malha) ou geométricas (malhas poliédricas móveis). A classe fvMesh é uma extensão da polyMesh que inclui dados adicionais necessários para a discretização por volumes finitos (SILVA, 2008).

Em adição, as variáveis de campo e seus contornos são alocados em posições específicas da malha como centro, faces ou vértices dos volumes de controle usando a classe geometricField $<$ Type $>$. Esta última é construída a partir das classes referentes à malha (fvMesh) e ao campo alocado no interior do contorno da geometria, respectivamente definidas pelas classes Field $<$ Type $>$ e geometricBoundaryField $<$ Type $>$. Note que a classe geometricBoundaryField $<$ Type $>$ herda as propriedades das classes com a qual é construída. De fato, esta classe é renomeada para distinguir a posição onde os campos são alocados na malha, definidos como:

- volField <Type $>$ - o campo está alocado no centro dos volumes de controle;

- surfaceField <Type $>$ - o campo está alocado nas superfícies dos volumes de controle;

- pointField $<$ Type $>$ - o campo está alocado nos vértices da malha.

Como citado anteriormente, a discretização das equações é realizada pelo método dos volumes finitos e as classes fvm e fvc são responsáveis pela aproximação dos termos derivativos das variáveis tensoriais calculadas (SILVA, 2008). Apesar destas classes possuírem o mesmo propósito, suas aplicações são diferentes. A classe fvm reúne funções para realizar operações implícitas de discretização, armazenando os resultados em uma matriz esparsa definida pela classe fvMatrix $<$ Type $>$. Em outras palavras, a classe fvm discretiza implicitamente os termos e constrói um sistema de equações lineares. Esta classe é capaz de realizar todas as operações que envolvem $\varphi^{n}$ colocadas na Tabela 3.1

Por outro lado, a classe fvc agrupa funções para calcular operações explícitas de discretização dos termos presentes nas equações. Assim, esta classe pode realizar cálculos explícitos diretos e seu resultado pode ser usado diretamente no código ou mesmo alocado no termo fonte do sistema linear. Estas operações de discretização envolvem as 
variáveis de campo no instante atual, $\varphi^{0}$, e retornam um campo geométrico geometricField $<$ Type $>$. O OpenFOAM fornece funções para realizar outras operações explícitas além daquelas colocadas na Tabela 3.1. como o rotacional de uma campo e média ponderada no volume de controle obtida a partir dos valores das faces.

A Fig. 3.4 mostra as diferenças no uso e resultados das classes fvm e fvc operando sobre um geometricField $<$ Type $>$. Uma vez que o sistema linear definido pela classe fvMatrix < Type $>$ é construído, este pode ser resolvido pela função de classe solve. Assim como os métodos e funções de interpolação usadas na discretização das equações, o método numérico para a solução do sistema linear é definido em tempo de execução. Assim, o usuário pode escolher livremente os métodos usados através dos arquivos de configuração simulado (mostrado na Seção 3.4).

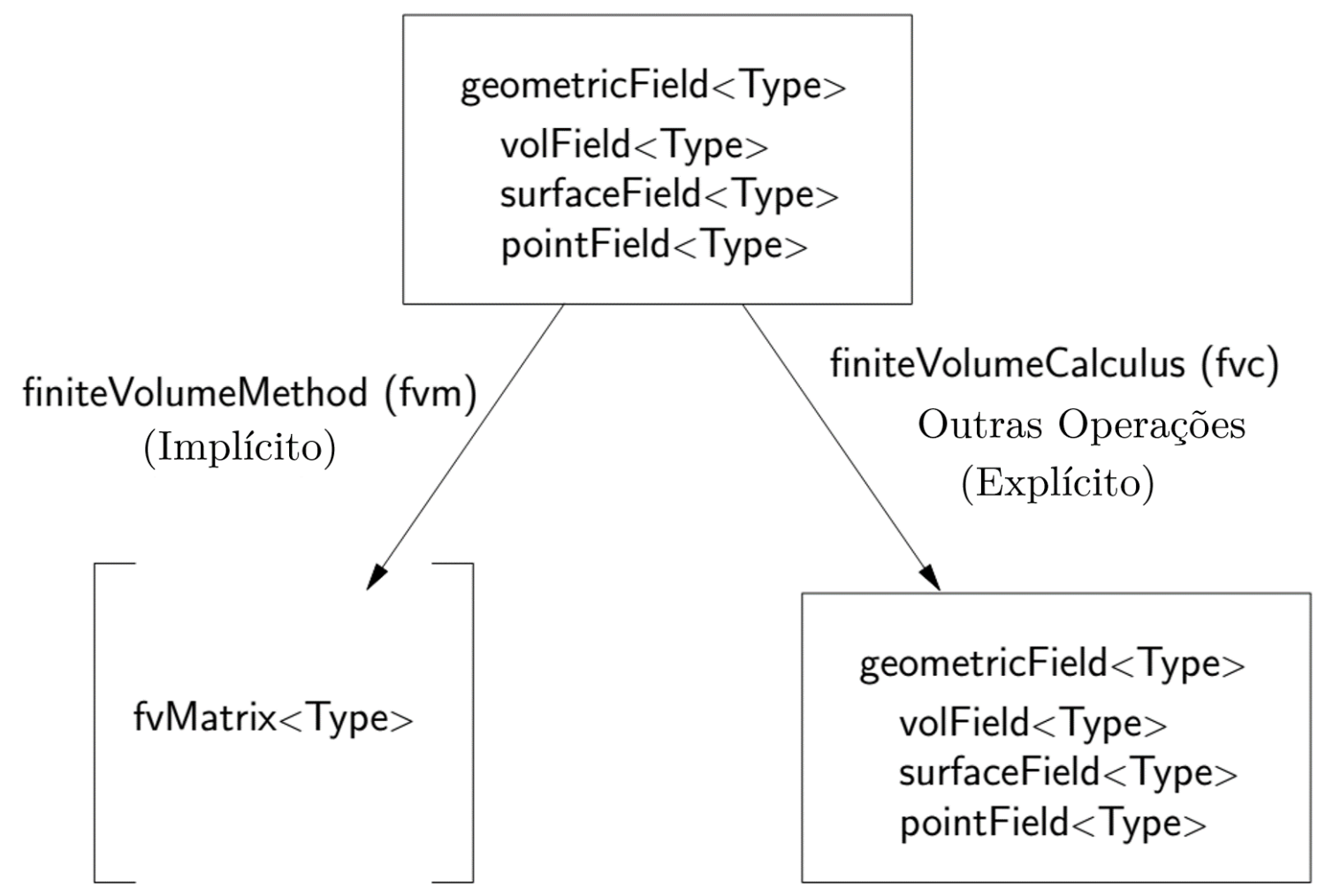

Figura 3.4: Operações da Classe geometricField < Type > usando Operações Implícitas (fvm) e Explícitas (fvc) (Adaptado de (OPENCFD, 2014a)). 


\subsection{Convecção Natural usando a Hipótese de Boussinesq no OpenFOAM}

O solver do OpenFOAM escolhido para resolver o problema de convecção, através da hipótese de Boussinesq é o buoyantBoussinesqPimpleFoam, este utiliza um conjunto de arquivos onde estão armazenadas as informações necessárias para a solução do problema (diretório de caso) que podem ser vistos na Fig. (3.5b). Estes arquivos se encontram num diretório e possuem as informações como a descrição da geometria, detalhes da malha, condições de contorno, parâmetros para os métodos numéricos e as propriedades físicas do problema, mostrados na Fig. (3.5a).

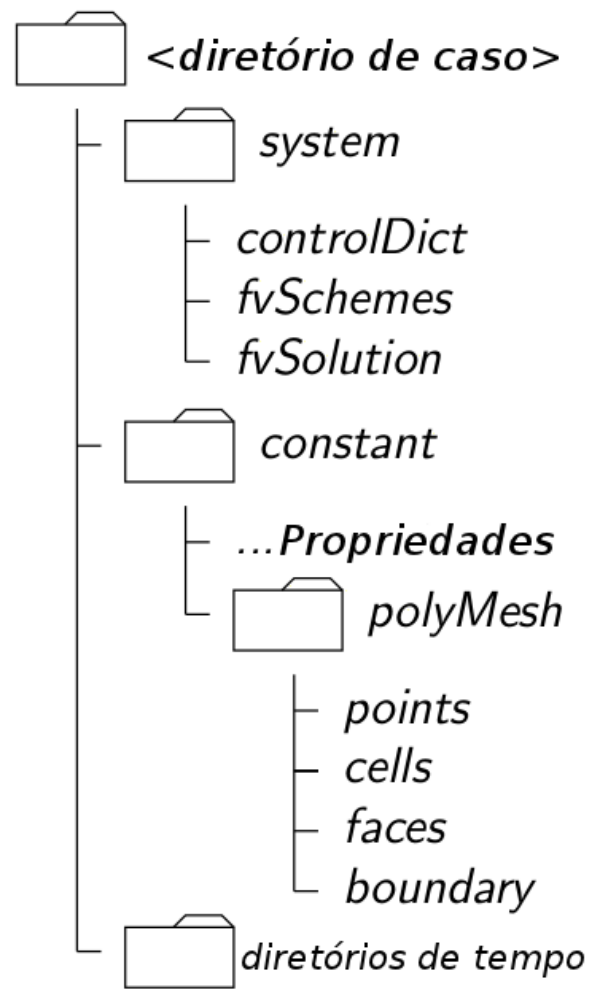

(a)

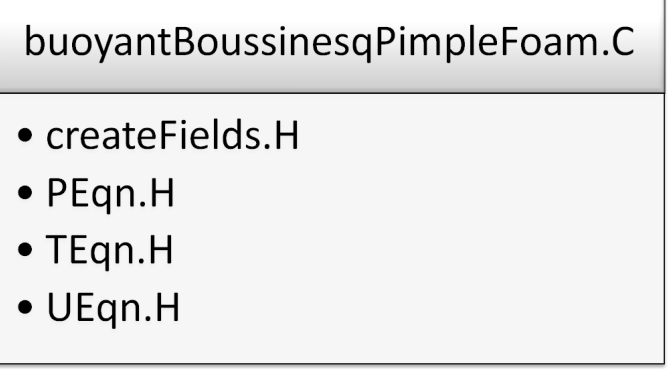

(b)

Figura 3.5: (a) Estrutura de Diretório de Caso (Adaptado de (OPENCFD, 2014b)); (b) Arquivos do Solver do OpenFOAM. 


\subsubsection{Estrutura Principal para Efetuar a Simulação no OpenFOAM}

O diretório principal < diretório de caso> é a "raiz" do caso e dentro deste estão incluídos os outros diretórios e arquivos de configuração. Uma breve descrição sobre o conteúdo destes diretórios é colocado na sequência.

- <diretório de tempo>: contém os arquivos individuais de dados para os campos das variáveis tratadas no caso (por exemplo, campo de velocidade, pressão, tensão, etc.). O nome associado ao diretório < diretório de tempo> refere-se ao instante simulado no qual os dados são escritos.

- <system>: os arquivos contidos neste diretório estão associados com o procedimento de solução do caso. Pelo menos 3 arquivos devem estar contidos em system: $(i)$ controlDict, onde se define os parâmetros de controle da simulação, como o tempo de início e término da simulação, passo de tempo, controle de escrita de dados, etc. (ii) fvSolution, que seleciona os métodos para resolver o sistema de equações lineares e suas tolerâncias, assim como outros parâmetros de controle do algoritmo de solução. (iii) fvSchemes seleciona as aproximações de discretização por volumes finitos usados na solução do problema. Conforme mostrado na Fig. 3.6 .

- <constant>: deve conter os arquivos de propriedades físicas pertinentes ao caso, por exemplo, transportProperties. A descrição completa da geometria e da malha deve ser incluída no diretório polyMesh, nos arquivos blockMeshDict e boundary. 


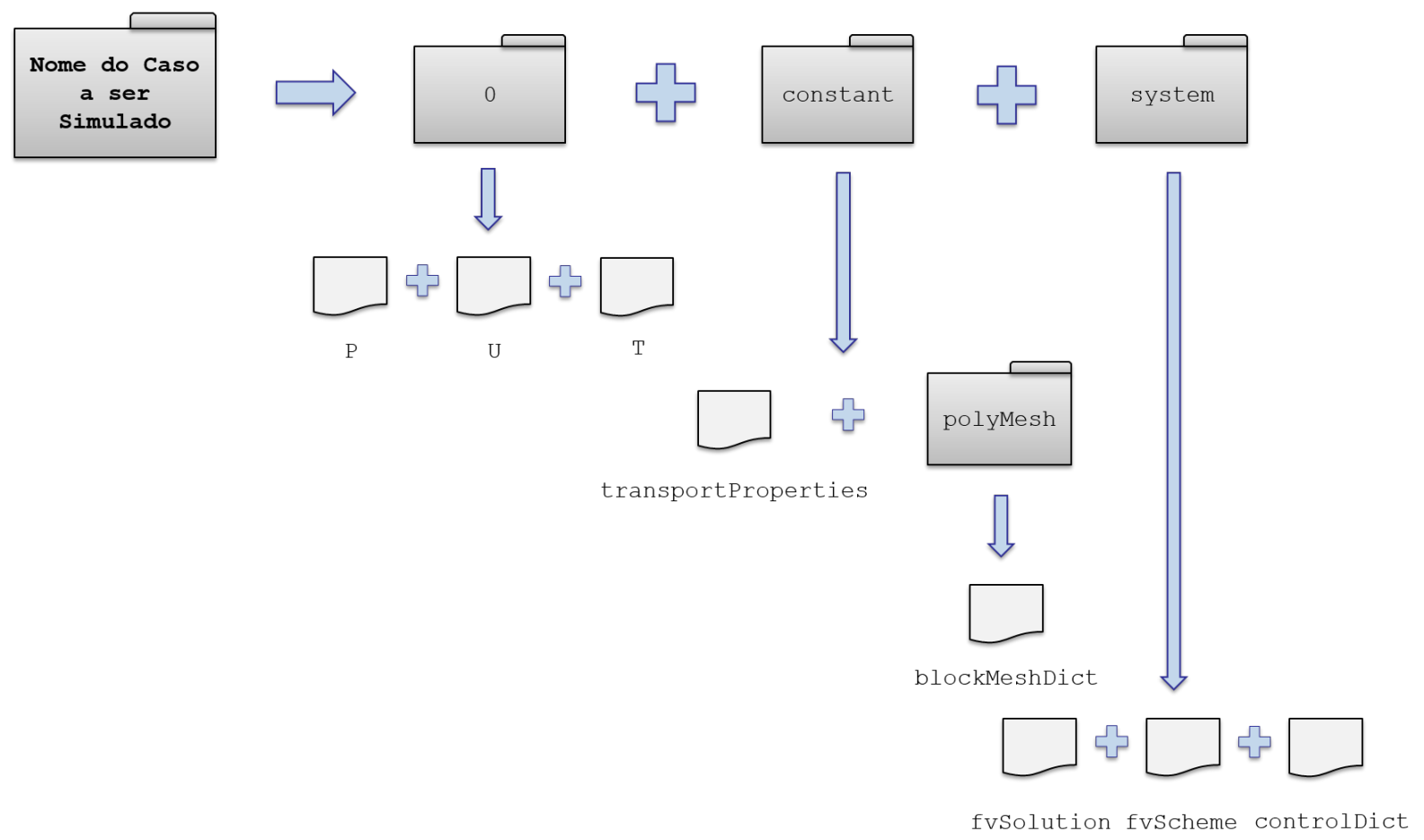

Figura 3.6: Estrutura de Arquivos do solver buoyantBouss inesqPimpleFoam

\subsubsection{Pré-Processamento}

\subsubsection{Construção da Geometria e da Malha}

O OpenFOAM não possui um editor CAD para construção da geometria do problema, que é armazenada no arquivo de configuração blockMeshDict. O princípio por trás da construção da geometria no OpenFOAM é a decomposição do domínio computacional em um conjunto de um ou mais blocos hexaédricos tridimensionais. As arestas dos blocos podem ser linhas retas ou arcos e cada bloco é definido por oito vértices (um para cada canto do hexágono). Os vértices são numerados e escritos em uma lista, formando pontos no espaço tridimensional. Geometrias mais complexas podem ser geradas pela combinação de mais blocos ou pelo colapso de um ou mais pares de vértices em outro. A construção da geometria é realizada através do conjunto das coordenadas dos vértices (ou arcos) de blocos colocadas no arquivo blockMeshDict.

O OpenFOAM tem a capacidade de gerar malhas estruturadas a partir das informa- 
ções sobre a geometria e condições de contorno do problema contidas nos arquivos de configuração blockMeshDict e boundary (presentes no subdiretório constant/polyMesh). Além de incluir os dados de geometria do problema, o arquivo blockMeshDict ainda contém as informações da malha como o número de células na discretização em cada eixo coordenado, a razão de expansão das células na malha (permite alterar o gradeamento da malha) e os patches presentes na geometria.

O comando blockMesh gera arquivos a partir do blockMeshDict e do boundary, estruturando os dados da malha em pontos, faces e conectividade entre as células (arquivos points, faces, owner e neighbour). Com estas informações, o solver é capaz de entender os dados de geometria e malha.

Contudo, optou-se por utilizar um gerador de malha externo ao OpenFOAM, a plataforma Salomé (The Open Source Integration Platform for Numerical Simulation), que inclui um editor CAD, um gerador de malha e outras ferramentas de pré e pósprocessamento. Após a construção da geometria e da malha o arquivo é exportado para o OpenFOAM e, então, gerados os arquivos necessários para a pasta do blockMeshDict.

\subsubsection{Configurações da Simulação}

Além da definição da geometria e da malha, o pré-processamento no OpenFOAM consiste na definição dos arquivos contendo o controle das condições da simulação e as propriedades físicas e modelos adicionais do problema.

Como já foi citado na Seção 3.4.1, as informações inclusas no arquivo controldict permitem controlar o tempo de simulação, passo de tempo, etc. Porém, outros arquivos de controle de simulação podem ser colocados no diretório system. Um deles é o controle das simulações em paralelo que é feito pelo arquivo decomposeParDict. O OpenFOAM usa a biblioteca de domínio público MPI (Message Passage Interface) para a comunicação entre os computadores e a decomposição do domínio pode ser feita por quatro métodos diferentes, onde o METIS ((KARYPIS; KUMAR, 1998a) e (KARYPIS; KUMAR, 1998b)) se destaca devido à grande eficiência no particionamento da malha.

Os métodos numéricos para a discretização dos termos derivativos das equações são definidos no arquivo de configuração fvSchemes. É imperativo que cada termo da 
equação esteja vinculado à uma aproximação numérica. O métodos de discretização padrão adotado pelo OpenFOAM é a integração de Gauss para volumes finitos. A integração de Gauss é baseada na soma dos fluxos das variáveis nas faces do volume, que devem ser interpolados a partir do centro dos volumes. O usuário pode escolher livremente o tipo de método de interpolação a ser usado. Dentre estes, pode-se citar as abordagens de interpolação linear (diferenças centrais), upwind, QUICK, MUSCL, TVD (Total Variation Diminishing) e NV (Gamma Normalised Variable). Para a integração no tempo, o OpenFOAM dispões de métodos de Euler explícito e CrankNicholson implementados em seu código. Mais informações sobre a metodologia de discretização utilizada pelo OpenFOAM pode ser encontrada em (MALISKA, 2004) e JASAK, 1996).

Nesta etapa também devem ser definidos os métodos de solução de equações lineares e suas respectivas tolerâncias, assim como alguns parâmetros para o algoritmo de solução de escoamento (correção pressão-velocidade e ortogonalidade da malha). Estas informações estão alocadas no arquivo de configuração fvSolution. Os métodos de solução de matrizes esparsas implementados no OpenFOAM são iterativos e, portanto, baseiam-se em reduzir o resíduo das equações até um valor pré-estabelecido ((MARIĆ; HÖPKEN; MOONEY, 2014)). Entre os algoritmos implementados no OpenFOAM, pode-se citar os métodos de Gauss-Seidel, Multigrid algébrico e variantes do gradiente conjudago. O método de solução é interrompido quando o resíduo se torna menor que a tolerância especificada (tol) e a razão entre os resíduos da iteração atual e inicial for menor que a tolerância relativa (<relTol >).

O algoritmo PISO (pressure-implicit split-operator) e SIMPLE (semi-implicit method for pressure-linked equations) estão implementados no OpenFOAM para resolver o acoplamento pressão-velocidade presente nas equações de escoamento de fluidos. Os dois algoritmos são baseados em procedimentos iterativos, avaliando a solução em dado instante de tempo e, então, corrigindo-a.

Por fim, deve-se definir as propriedades físicas e os modelos adicionais na simulação em arquivos específicos para cada caso. 


\subsubsection{Solução Numérica}

As simulações no OpenFOAM são realizadas por arquivos executáveis chamados solver que leem as informações referentes ao caso (malha e condições de simulação) e resolvem problemas específicos de mecânica do contínuo. Na essência, os solvers são resultado da compilação dos arquivos fonte e a solução numérica depende de como as bibliotecas do OpenFOAM são usadas para montar o algoritmo de solução.

O solver utilizado na validação da malha (Seção 3.4.2.1) é chamado de buoyantBoussinesqPimpleFoam. Este solver utiliza da hipótese de Boussinesq para resolver o problema de convecção natural transiente com o algoritmo PIMPLE (junção do algoritmo PISO e SIMPLE) para resolver o problema de acoplamento pressãovelocidade.

Na Figura 3.7 há uma ilustração dos arquivos que compõem o solver, assim como das bibliotecas auxiliares utilizadas na solução numérica. $O$ arquivo principal que tem o mesmo nome do solver é buoyantBoussinesqPimpleFoam. C este códigofonte é responsável pela organização do processo de simulação em que a biblioteca createFields. H é usada para leitura e criação de campos iniciais para as variáveis do problema e propriedades físicas pertinentes à convecção natural. Na sequência são resolvidos os campos de velocidade e temperatura pelos arquivos UEqn. H e TEqn. H, respectivamente. Depois disso, o loop de correção (executado pelo algoritmo PIMPLE) é introduzido e corretores de pressão e velocidade são executados utilizando o código em pEqn. H. Maiores detalhes serão dados nas implementações numéricas (Seção 3.5 e Apêndice A.

\subsubsection{Pós-Processamento}

O OpenFOAM possui uma ferramenta para o pós-processamento dos resultados que é denominada de ParaFoam, adaptada do programa ParaView para visualização científica de código aberto. Este, por sua vez, é baseado no VTK que é um programa de software livre para o processamento de dados e renderização de imagens.

As ferramentas básicas para visualização de resultado CFD estão incluídas no paraF oam, como a criação de gráficos de contorno, vetores e linhas de fluxo, superfícies, animações, 
etc.

\subsection{Implementação Numérica do Solver para o Problema de Agitação da Cavidade}

Conforme citado na Seção 3.4.3, o OpenFOAM dispõe de um solver padrão para problemas de convecção natural transiente, baseado na hipótese de Boussinesq. Porém, o solver traz somente as informações básicas para uma simulação simples. O objetivo é criar um novo solver usando como referência o buoyantBoussinesqPimpleFoam, de maneira que possa incluir o processo de agitação e/ou rotação.

Inicialmente, fizemos um processo de aproveitamento da estrutura do solver original buoyantBoussinesqPimpleFoam, localizado na pasta \applications \solvers \heatTransfer do OpenFOAM. Toda a estrutura do arquivo principal, arquivos auxiliares e bibliotecas foi aproveitada e o novo solver passou a se chamar g_buoyantBoussinesqPimpleFoam em alusão à gravidade.

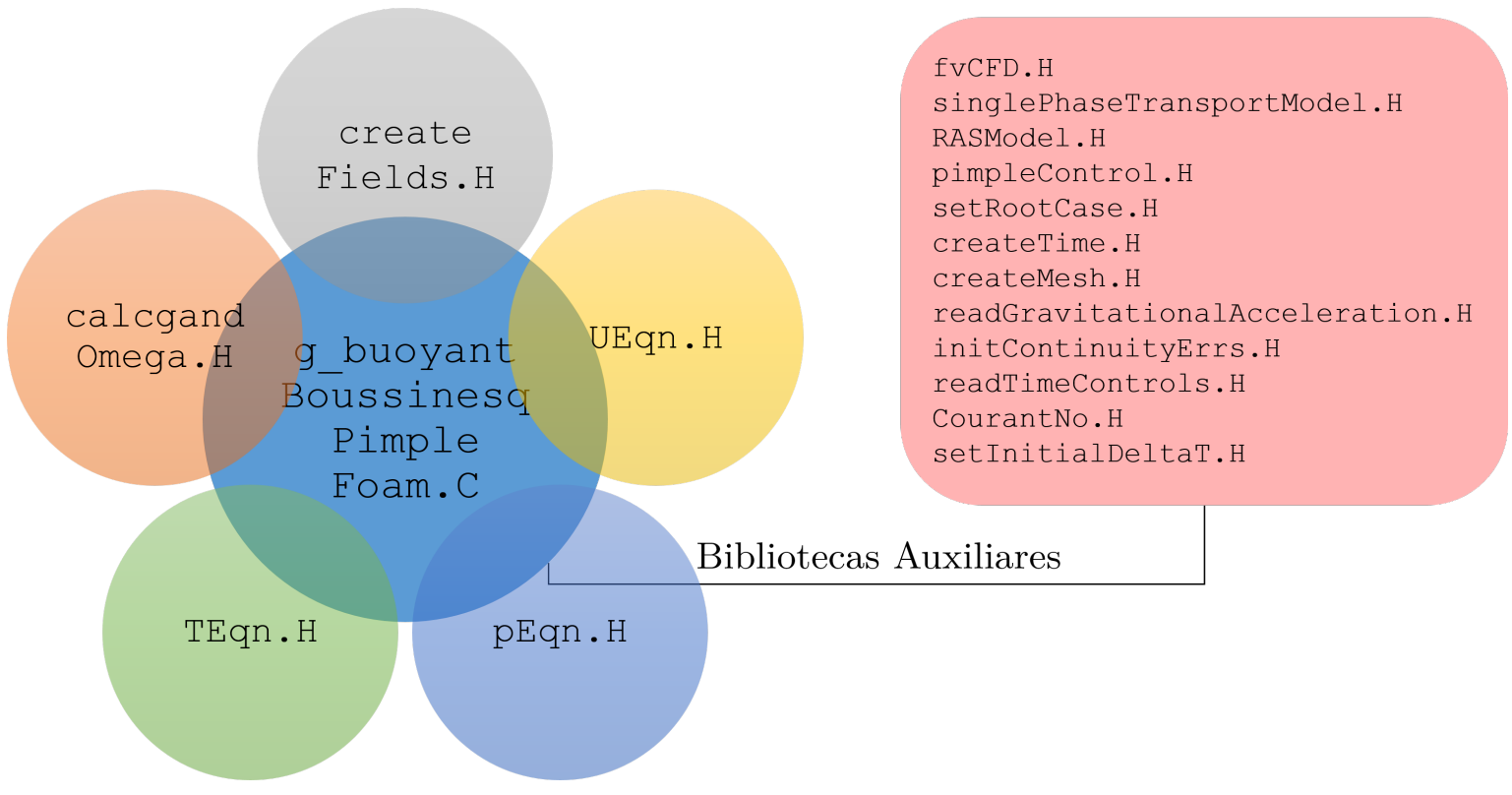

Figura 3.7: Conteúdo do solver buoyantBouss inesqP impleFoam

O código fonte em $\mathrm{C}++$ para resolver esse problema está no arquivo principal g_buo- 
yantBoussinesqPimpleFoam. H dado pelo Código A.1. A primeira linha do código declara a biblioteca fVCFD. H que é a principal biblioteca do OpenFOAM e que permite acesso a todas as classes. Essa biblioteca é necessária em todos os solvers. Na segunda linha é declarada a biblioteca singlePhaseTransportModel. H que seleciona o modelo de transporte para o tipo de fluido. Na sequência, temos a escolha do modelo de turbulência com RASModel. H (para o presente trabalho o escoamento é laminar), a inclusão do método de acoplamento pimpleControl.H e as constantes matemáticas utilizadas nos cálculos (mathematicalconstants.H).

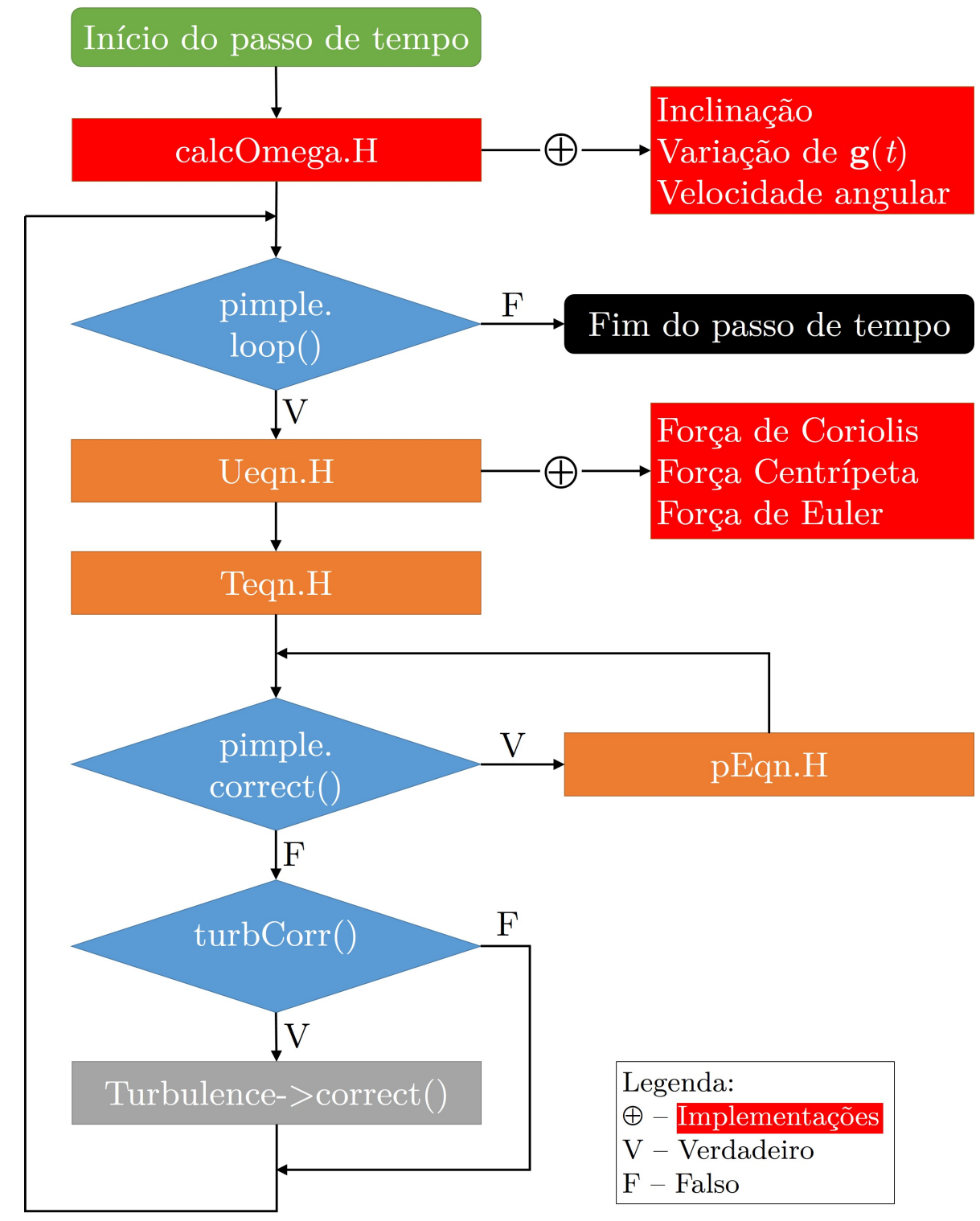

Figura 3.8: Representação Simplificada da Execução do solver buoyantBoussinesqPimpleFoam e as Implementações 
Na sequência, os arquivos do novo solver são alterados para incluir as informações dadas na formulação matemática que foi mostrada na Seção 2.4.2.

Dentre as principais mudanças, está a inclusão do arquivo auxiliar ca l cgandOmega • H (Código A.3) que tem como objetivo estabelecer o cálculo do vetor velocidade angular $(\Omega)$, com base no ângulo $\theta(t)$ :

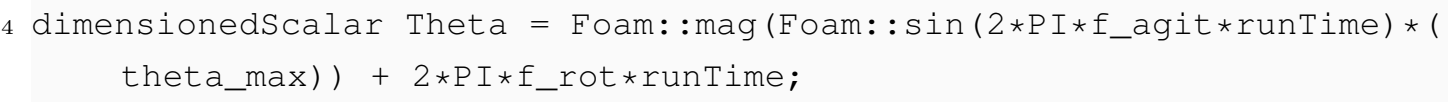

do vetor gravidade $\mathbf{g}$

$11 \mathrm{~g}=$ gunits $\star$ Foam: $: \sin ($ Theta $) * \operatorname{vector}(1,0,0)-$ gunits*Foam: : cos $($ Theta $) *$ vector $(0,0,1)$;

e da alteração do centro de referência para a rotação e/ou agitação:

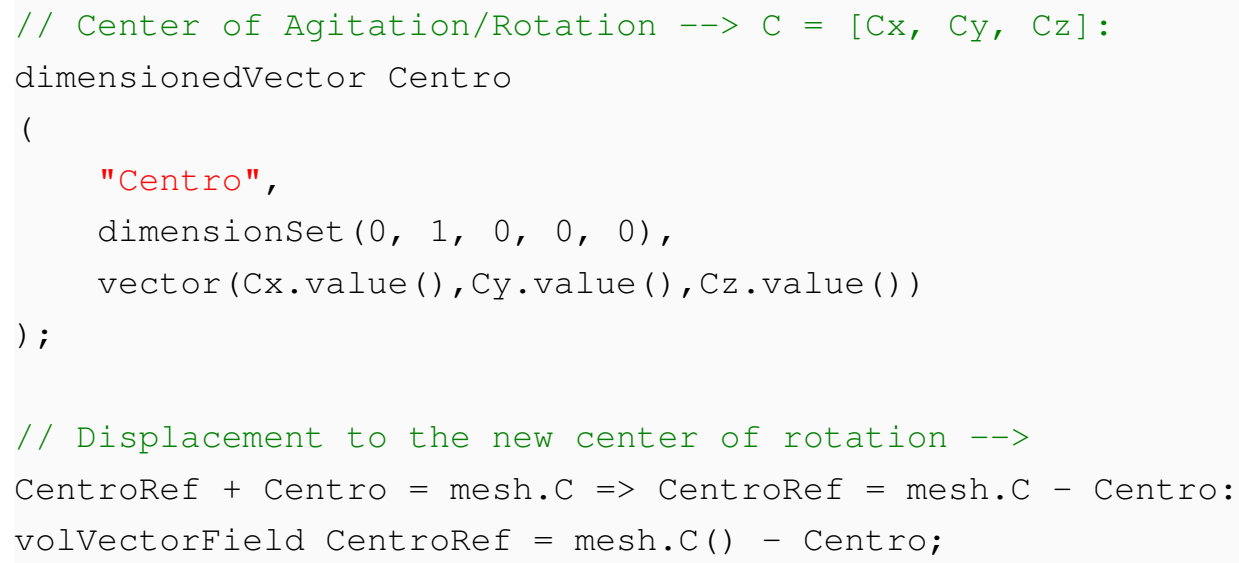

Esses cálculos auxiliares são chamados no arquivo principal pela biblioteca createFields.H (Código A.2) que geram os campos iniciais para as variáveis do problema e propriedades físicas pertinentes ao caso $\left(T, p_{r g h}, U, \Omega, \kappa_{t}, p, f_{\text {rot }}, f_{\text {agit }}, \theta_{\max } \mathrm{e}\right.$ $\left.\left(C_{x}, C_{y}, C_{z}\right)\right)$ e é específico para cada solver. 
Em seguida é montada a matriz para o cálculo implícito da variável $U$ pelo arquivo UEqn. H, devido a grande estabilidade do método, o que significa que um conjunto de equações lineares acopladas, expressadas em um sistema linear da forma $\mathcal{A} \mathbf{x}=\mathbf{b}$ são resolvidas. O lado esquerdo da equação é resolvido pelo trecho do Código A.4.

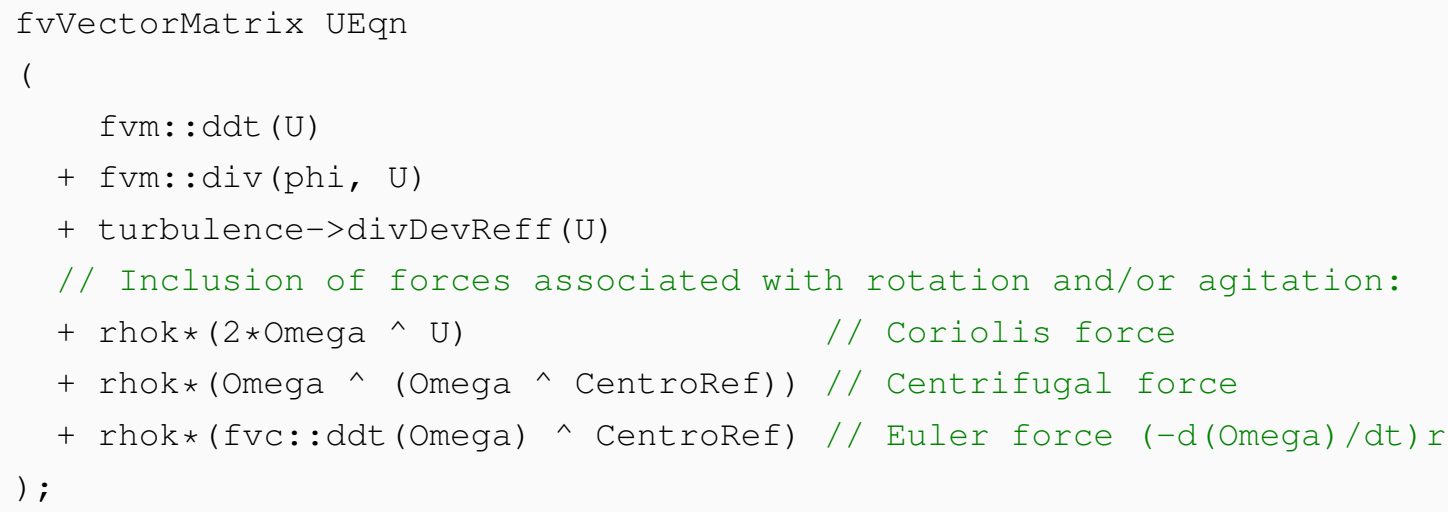

em que rhok $\left(\rho_{k}\right)$ é a densidade efetiva, dado por $\rho_{k}=1-\beta\left(\bar{T}-T_{0}\right)$ e que utiliza da aproximação de Boussinesq, que será calculado no arquivo para TEqn.H.

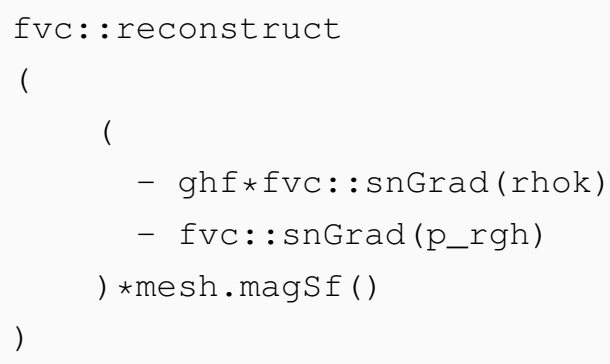

Conforme mencionado anteriormente, a classe padrão para a montagem da matriz de volumes finitos é a fvm e é utilizada quando tem-se operações que são implícitas e para formarmos o lado esquerdo da matriz. Na linha 3 temos o termo correspondente à primeira derivada no tempo da velocidade, fvm: : ddt (U), na sequência, + fvm: : div (phi, U), o termo divergente e, logo abaixo, nas linhas 9, 10 e 11, incluem, respectivamente, as forças de Coriolis, Centrífuga e de Euler. O lado direito é montado a partir da linha 22 em que temos o termo de gravidade e de pressão.

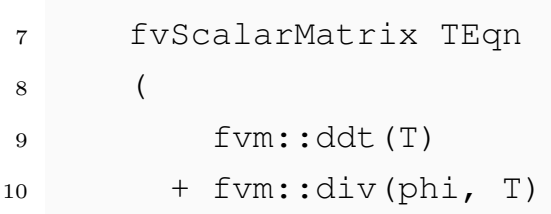



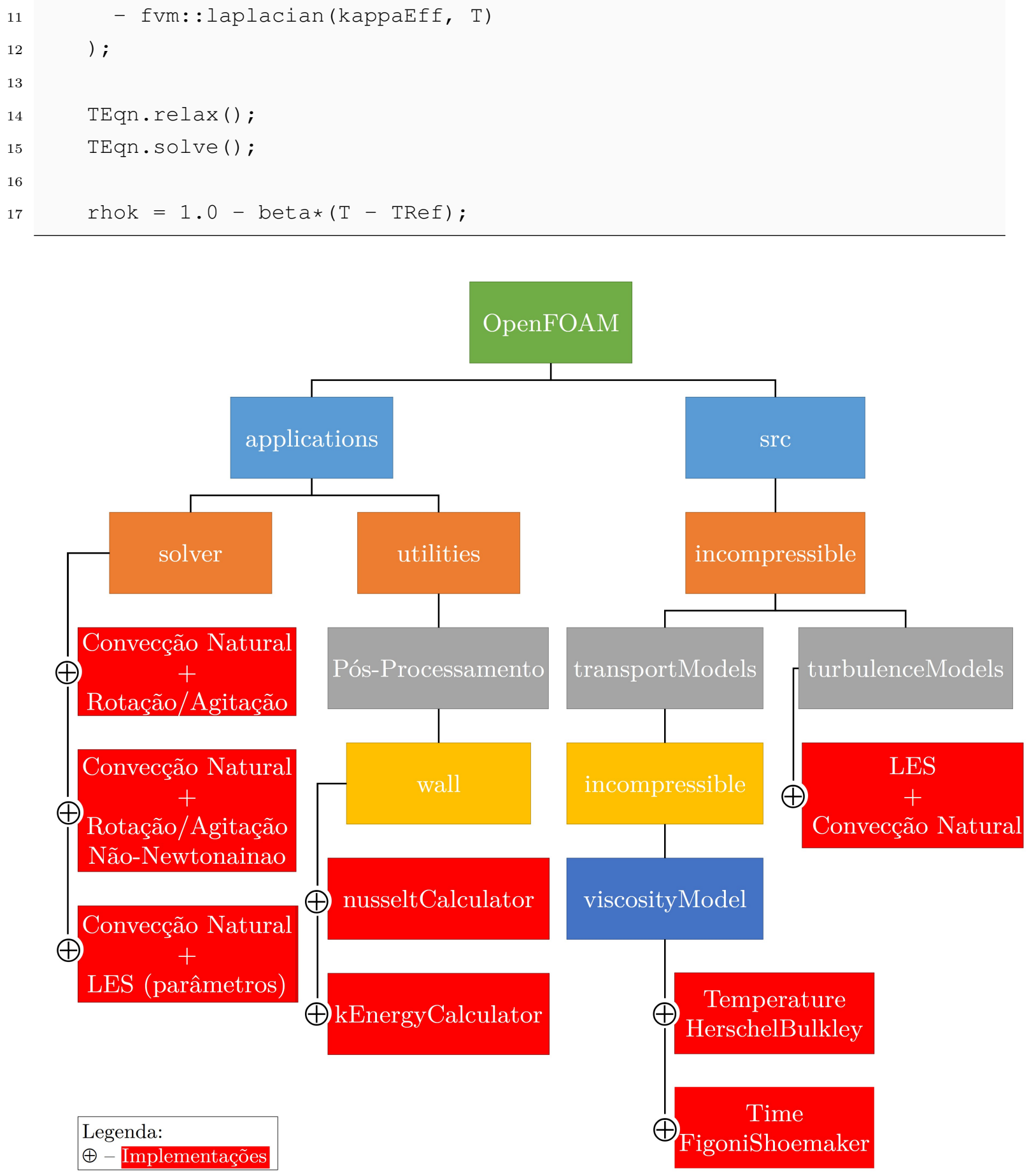

Figura 3.9: Representação dos solvers, Alteração no Método de LES e Inclusão de Aplicações para o Cálculo de $\mathrm{Nu}$ e K. 
4. Resultados

\section{Capítulo 4}

\section{Resultados}

\subsection{Introdução}

Neste capítulo apresentaremos os resultados da simulação da convecção natural de uma cavidade paralelepípeda retangular preenchida com água e etileno glicol para vários valores de Rayleigh em condições específicas de temperatura de aquecimento e de geometria. São avaliadas, também, as características do comportamento da convecção natural em situações em que a cavidade é agitada, com a finalidade de obter uma aceleração no aquecimento.

Duas geometrias foram utilizadas para analisar o comportamento da convecção natural na cavidade: uma paralelepípeda retangular e outra cúbica, ambas apresentadas na figura $4.1 \mathrm{a})$ e (4.1 $\mathrm{b}$ ), respectivamente. A origem dos eixos encontra-se no centro da cavidade e pode ter seu ângulo de inclinação $(\phi)$ variando com o tempo, consequentemente, o vetor gravidade (g) também dependerá de $t$. 


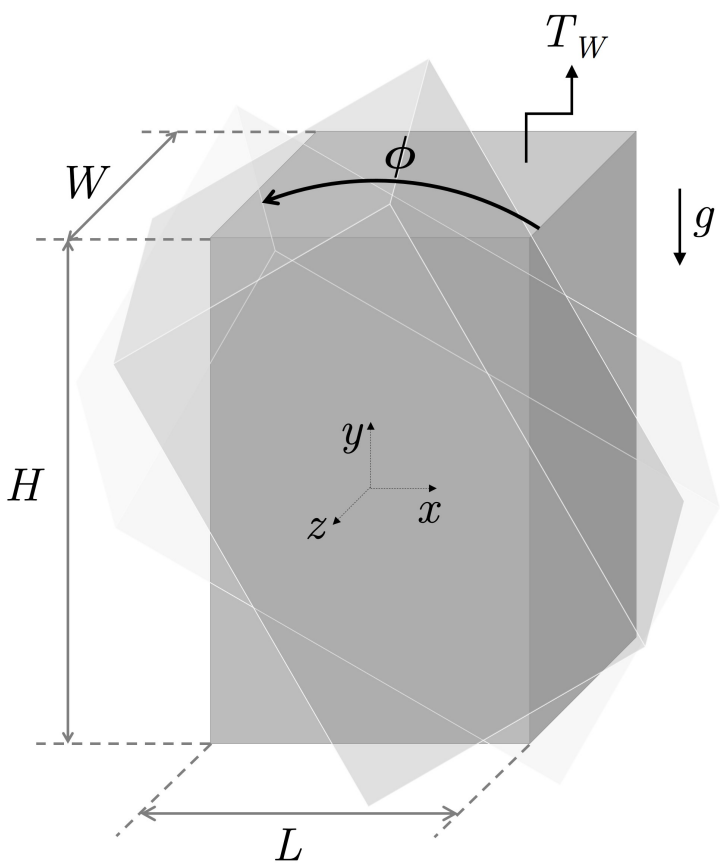

(a)

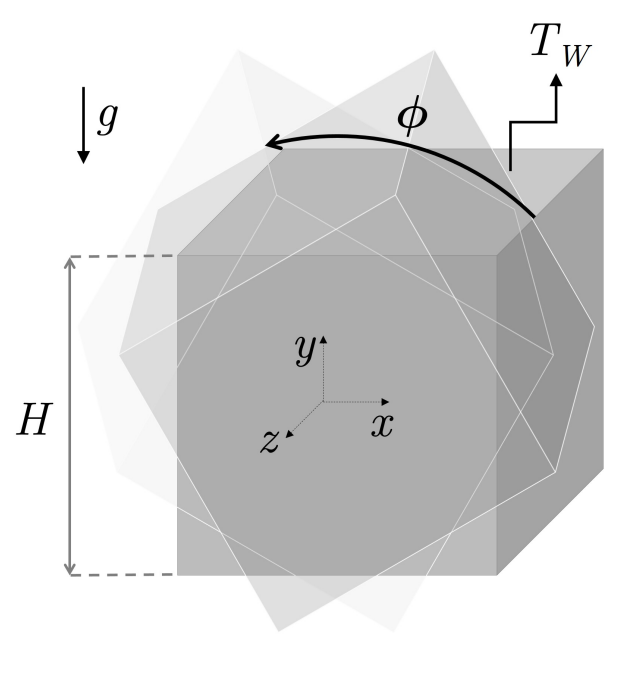

(b)

Figura 4.1: Geometrias Utilizadas nos Estudos de Casos para a Convecção Natural: (a) Paralelepípedo Retangular; (b) Cubo.

As primeiras simulações foram para validação da capacidade do OpenFOAM em prever resultados satisfatórios embasadas nas informações obtidas em trabalhos experimentais de (LIN; AKINS, 1983) e (TOLLINI, 1996) para valores Nusselt versus Rayleigh e velocidade no centro do cubo (Tabela 4.1). 


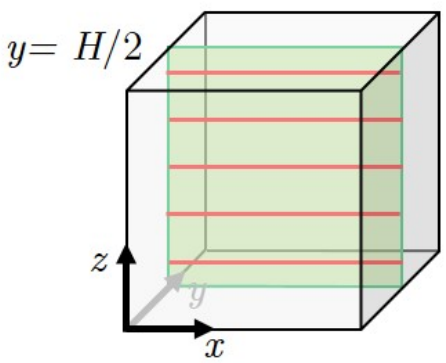

(a)

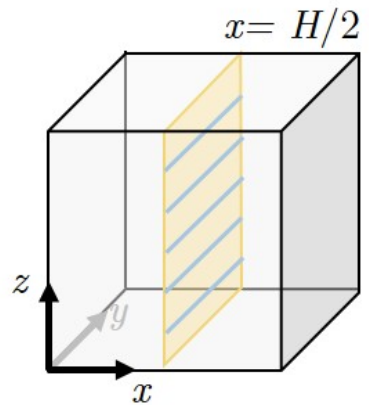

(b)

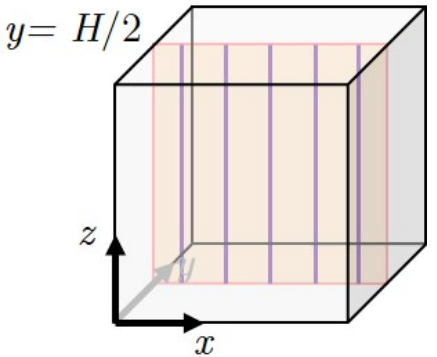

(c)

Figura 4.2: Posicionamentos dos Locais de Extração nas Linhas sobre os Planos: (a) $y=H / 2($ para $z=29 H / 30, z=3 H / 4, z=H / 2, z=H / 4$ e $z=H / 30)$; (b) $x=H / 2$ (para $z=29 H / 30, z=3 H / 4, z=H / 2, z=H / 4$ e $z=H / 30)$; (c) $y=H / 2($ para $x=29 H / 30$, $x=3 H / 4, x=H / 2, x=H / 4$ e $x=H / 30)$.

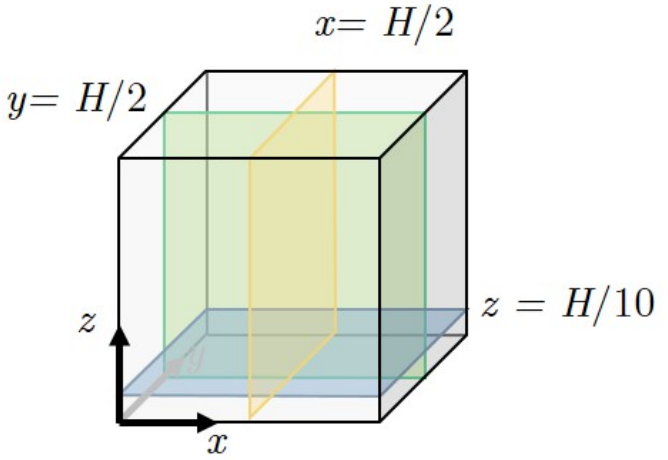

(a)

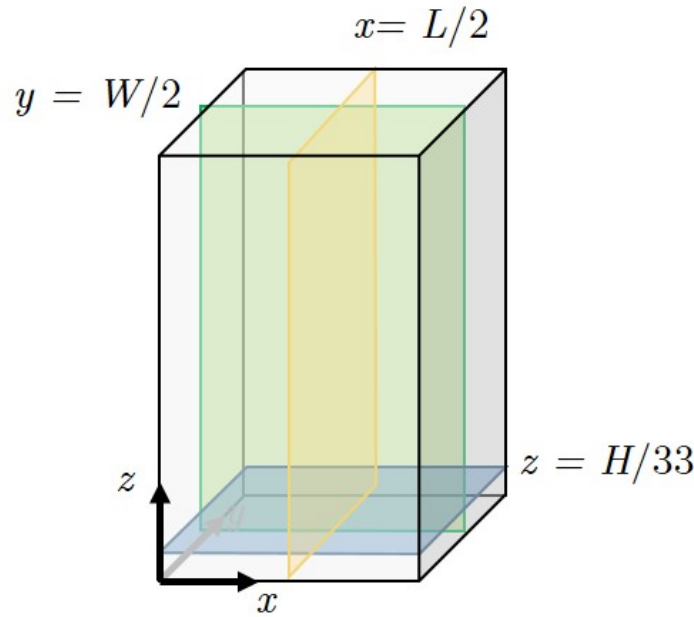

(b)

Figura 4.3: Planos de Visualização dos Resultados para Contornos e Vetores: (a) Cubo; (b) Paralelepípedo Retangular 


\begin{tabular}{|c|c|c|c|c|c|c|c|c|c|c|c|c|c|c|}
\hline \multirow{6}{*}{ 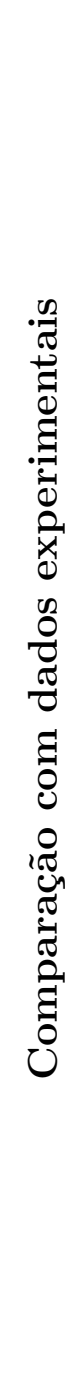 } & \multirow{3}{*}{ 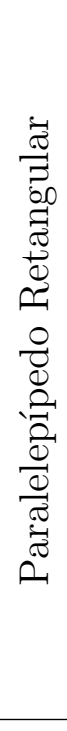 } & \multirow{6}{*}{$\frac{\pi}{\overrightarrow{8} 0}$} & $\begin{array}{l}0 \\
\stackrel{0}{\infty} \\
\stackrel{8}{2}\end{array}$ & 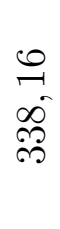 & \multirow{3}{*}{$\begin{array}{l}10 \\
\stackrel{10}{0} \\
0\end{array}$} & \multirow{8}{*}{$\begin{array}{l}\bar{\infty} \\
\infty\end{array}$} & $\begin{array}{l}\overrightarrow{1} \\
0 \\
0 \\
\dot{1} \\
\dot{0} \\
\dot{v}\end{array}$ & $\begin{array}{l}89 \\
0 \\
0\end{array}$ & 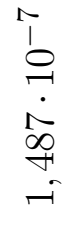 & $\begin{array}{l}\uparrow \\
\circ \\
\ddots \\
\dot{8} \\
\infty \\
\dot{0}\end{array}$ & 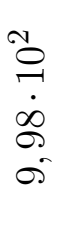 & $\begin{array}{l}\stackrel{8}{\circ} \\
\dot{1} \\
\dot{\infty} \\
\infty \\
\text { o }\end{array}$ & 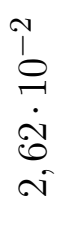 & $\underset{ت}{\rightleftarrows}$ \\
\hline & & & $\begin{array}{l}0 \\
\stackrel{-}{2} \\
\stackrel{2}{\Omega}\end{array}$ & $\begin{array}{l}\stackrel{0}{-1} \\
\infty \\
\infty \\
\infty\end{array}$ & & & $\begin{array}{l}\overrightarrow{1} \\
\dot{0} \\
\dot{\ddots} \\
\dot{8} \\
\dot{v}\end{array}$ & $\begin{array}{l}80 \\
0 \\
0\end{array}$ & 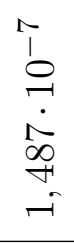 & $\begin{array}{l}1 \\
1 \\
0 \\
\ddots \\
\dot{0} \\
\infty \\
0\end{array}$ & $\begin{array}{l}\stackrel{0}{0} \\
\dot{1} \\
\dot{\infty} \\
0 \\
0\end{array}$ & $\begin{array}{l}8 \\
\stackrel{0}{1} \\
\dot{0} \\
10 \\
-1\end{array}$ & 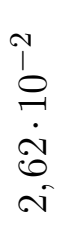 & $\begin{array}{l}0 \\
0 \\
0\end{array}$ \\
\hline & & & $\begin{array}{l}\stackrel{0}{-} \\
\stackrel{+}{S}\end{array}$ & $\begin{array}{l}0 \\
\stackrel{1}{0} \\
\infty \\
\infty \\
\infty\end{array}$ & & & 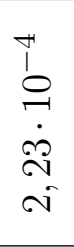 & $\begin{array}{l}-1 \\
0 \\
0\end{array}$ & 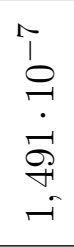 & $\begin{array}{l}1 \\
\mathfrak{o} \\
\ddots \\
\dot{0} \\
\dot{0} \\
0\end{array}$ & 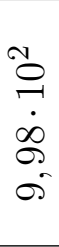 & $\begin{array}{l}\infty \\
\stackrel{0}{-} \\
\stackrel{1}{\circ} \\
\infty \\
\infty \\
0\end{array}$ & 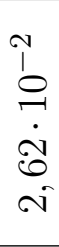 & $\begin{array}{l}\infty \\
\infty \\
0\end{array}$ \\
\hline & \multirow{5}{*}{$\frac{8}{3}$} & & \multirow{3}{*}{ 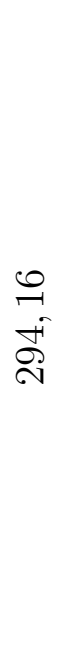 } & $\begin{array}{l}8 \\
\stackrel{8}{0} \\
\stackrel{2}{9}\end{array}$ & $\begin{array}{l}0 \\
0 \\
0 \\
-1 \\
0\end{array}$ & & \multirow{3}{*}{$\begin{array}{l}\overrightarrow{1} \\
1 \\
0 \\
\dot{1} \\
\dot{0} \\
\overrightarrow{1} \\
\dot{v}\end{array}$} & \multirow{3}{*}{$\begin{array}{l}F \\
0 \\
0\end{array}$} & \multirow{3}{*}{$\begin{array}{l}\hat{1} \\
\circ \\
\ddots \\
\dot{f} \\
\stackrel{f}{-i}\end{array}$} & \multirow{3}{*}{$\begin{array}{l}\hat{1} \\
0 \\
\ddots \\
\dot{0} \\
\hat{0} \\
\dot{0}\end{array}$} & \multirow{3}{*}{$\begin{array}{l}\mathscr{O} \\
\ddot{G} \\
\dot{\infty} \\
\mathscr{\infty} \\
\dot{\infty}\end{array}$} & $\begin{array}{l}\stackrel{8}{0} \\
\stackrel{-}{1} \\
\dot{N} \\
\stackrel{2}{N}\end{array}$ & $\begin{array}{l}0 \\
i \\
0 \\
\dot{1} \\
\infty \\
\infty \\
\infty\end{array}$ & $\begin{array}{l}p \\
i \\
\stackrel{1}{1} \\
\dot{8} \\
\dot{8}\end{array}$ \\
\hline & & & & 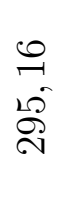 & 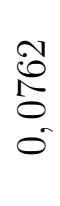 & & & & & & & $\begin{array}{l}\mathscr{D} \\
\stackrel{1}{1} \\
\dot{0} \\
0 \\
0\end{array}$ & 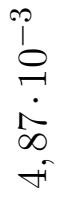 & 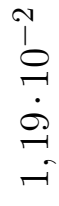 \\
\hline & & & & 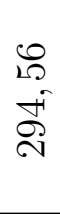 & $\begin{array}{l}\infty \\
0 \\
2 \\
0 \\
0 \\
0\end{array}$ & & & & & & & 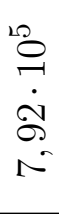 & $\begin{array}{l}p \\
i \\
\stackrel{0}{1} \\
\dot{\sim} \\
i 0 \\
i\end{array}$ & $\begin{array}{l}p \\
1 \\
0 \\
\dot{0} \\
\dot{0} \\
\dot{0}\end{array}$ \\
\hline $\begin{array}{l}\frac{0}{0} \\
\frac{\sigma}{0} \\
\frac{0}{0} \\
\frac{0}{0}\end{array}$ & & 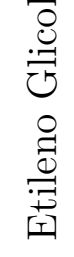 & $\begin{array}{l}0 \\
\stackrel{1}{\circ} \\
\stackrel{2}{\leftrightarrow}\end{array}$ & $\begin{array}{l}\stackrel{0}{-1} \\
\stackrel{-}{s} \\
\stackrel{5}{N}\end{array}$ & 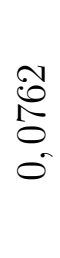 & & $\begin{array}{l}\overrightarrow{1} \\
0 \\
0 \\
\dot{10} \\
\overrightarrow{1} \\
\dot{0}\end{array}$ & $\begin{array}{l}\vec{\infty} \\
\stackrel{\infty}{\infty}\end{array}$ & $\begin{array}{l}\hat{1} \\
0 \\
\ddots \\
\dot{J} \\
\dot{f}\end{array}$ & $\begin{array}{l}0 \\
1 \\
0 \\
\ddots \\
\dot{1} \\
0 \\
0\end{array}$ & $\begin{array}{l}\stackrel{8}{\circ} \\
\stackrel{-}{\infty} \\
\infty \\
-1 \\
-1\end{array}$ & $\begin{array}{l}\stackrel{0}{\circ} \\
\stackrel{-}{-} \\
\dot{\sigma} \\
-1\end{array}$ & $\begin{array}{l}\stackrel{p}{b} \\
0 \\
\dot{0} \\
\dot{0} \\
0 \\
\sim\end{array}$ & $\begin{array}{l}\ddot{1} \\
0 \\
\dot{\theta} \\
\dot{\theta} \\
\dot{f}\end{array}$ \\
\hline 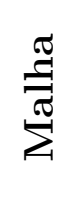 & & $\frac{\pi}{\overrightarrow{5}}$ & $\begin{array}{l}0 \\
\stackrel{1}{+} \\
\stackrel{S}{N}\end{array}$ & 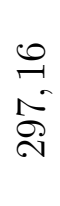 & $\begin{array}{l}0 \\
\stackrel{0}{0} \\
-1 \\
0\end{array}$ & & $\begin{array}{l}\overrightarrow{1} \\
0 \\
0 \\
\dot{0} \\
\dot{1} \\
\dot{v}\end{array}$ & $\begin{array}{l}-1 \\
0 \\
0\end{array}$ & $\begin{array}{l}\hat{1} \\
0 \\
\ddots \\
\dot{f} \\
\stackrel{-}{-}\end{array}$ & $\begin{array}{l}\hat{1} \\
\circ \\
\stackrel{1}{0} \\
\dot{a} \\
\dot{a}\end{array}$ & 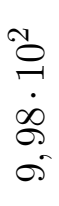 & 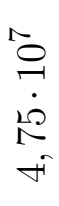 & $\begin{array}{l}p \\
1 \\
0 \\
\dot{1} \\
\dot{\rho} \\
1 \\
0 \\
0\end{array}$ & $\begin{array}{l}\stackrel{\imath}{b} \\
\stackrel{0}{0} \\
\dot{\vec{L}} \\
\dot{f}\end{array}$ \\
\hline 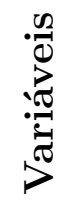 & 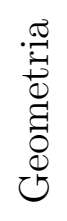 & $\frac{?}{.0}$ & $\underset{E}{E}$ & $\stackrel{E}{E}$ & $\underset{\Sigma}{\Sigma}$ & $\frac{\sqrt{\infty}}{\hat{E}}$ & $\begin{array}{l}\overrightarrow{1} \\
\frac{1}{2} \\
n\end{array}$ & $\overrightarrow{D_{1}}$ & $\frac{\sqrt{\infty}}{\frac{\sqrt{\sigma}}{\sigma}}$ & 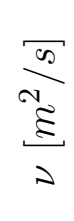 & 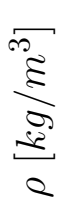 & $\stackrel{*}{*}$ & $\frac{\omega}{\xi}$ & \begin{tabular}{l} 
No \\
No \\
है \\
0 \\
\multirow{1}{*}{}
\end{tabular} \\
\hline
\end{tabular}




\subsection{Comparação com Dados Experimentais}

Três configurações de malha foram construídas para uma cavidade cúbica de 4 polegadas de lado $(H=0,1016 \mathrm{~m})$, baseada nas dimensões do trabalho experimental de $(\overline{L I N}, 1982)$ e (LIN; AKINS, 1983): $\left.1^{\mathrm{a}}\right) 256.000$ elementos; $\left.2^{\mathrm{a}}\right) 729.000$ elementos e $\left.3^{\mathrm{a}}\right)$ 1.728.000 elementos. Correspondendo, respectivamente, a 60, 90 e 120 elementos por aresta. Uma ilustração de uma das malha utilizada nos testes pode ser vista na Fig. 4.4 .

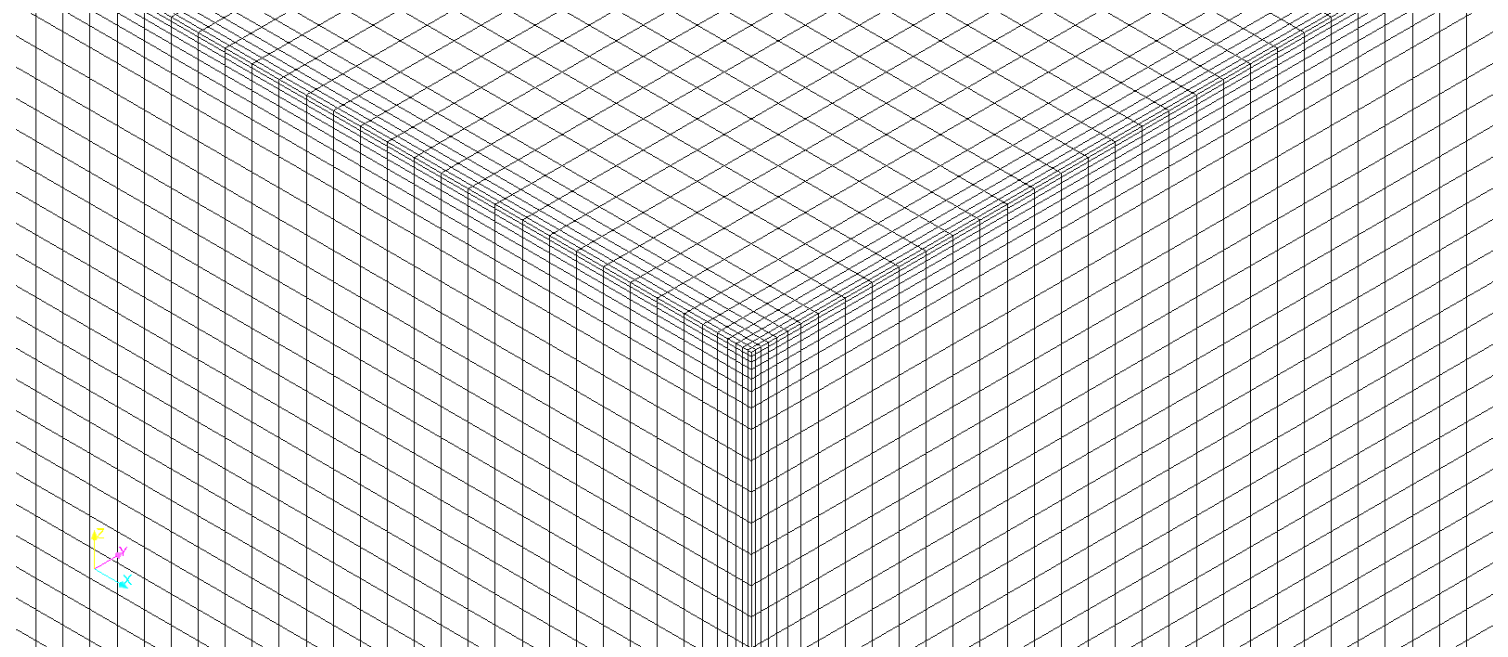

Figura 4.4: Detalhe da Malha Utilizada no Teste de Validação.

As simulações para o teste de malha tiveram a cavidade preenchida com água, a uma temperatura inicial, $T_{0}$, de $294 \mathrm{~K}$ e todas as paredes aquecidas a uma temperatura $T_{W}$ de $297 \mathrm{~K}$ e considerando $\phi=0$ (Seção 2.4.1). Com isso, temos como referência o número de Rayleigh global da ordem de $\mathrm{Ra}=4,75 \cdot 10^{7}$.

Tabela 4.2: Tempo de Execução

\begin{tabular}{ccc}
\hline Tamanho da Malha & Tempo de Execução & Tempo Total \\
\hline $60^{3}$ & $48.211,7 s \equiv 13,4 h \equiv 0,558 d$ & $48307 s \equiv 13,42 h \equiv 0,559 d$ \\
$90^{3}$ & $174.463,89 s \equiv 48,46 h \equiv 2,02 d$ & $174.671 s \equiv 48,52 h \equiv 2,02 d$ \\
$120^{3}$ & $445.982,6 s \equiv 123,88 h \equiv 5,16 d$ & $447.035,2 s \equiv 124,17 h \equiv 5,17 d$ \\
\hline
\end{tabular}

A Tabela 4.2 mostra os tempos de execução de cada configuração de malha. Obviamente, o aumento do número de elementos acarreta em um maior tempo de simulação, 
variando de 0,558, 2,02 e 5,16 dias, correspondendo ao tamanho de malha de $60^{3}, 90^{3}$ e $120^{3}$.

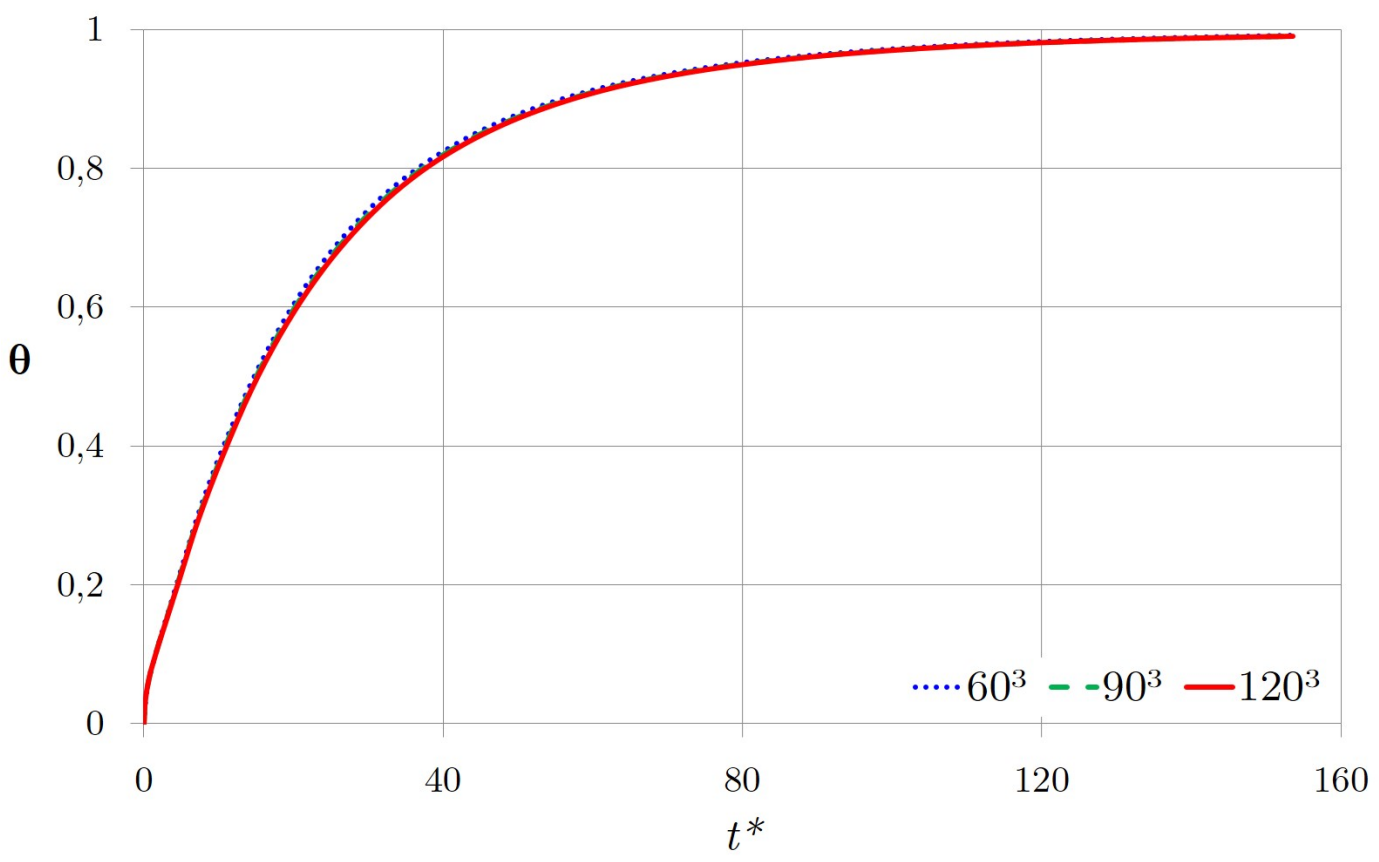

(a) $\theta \times t^{*}$

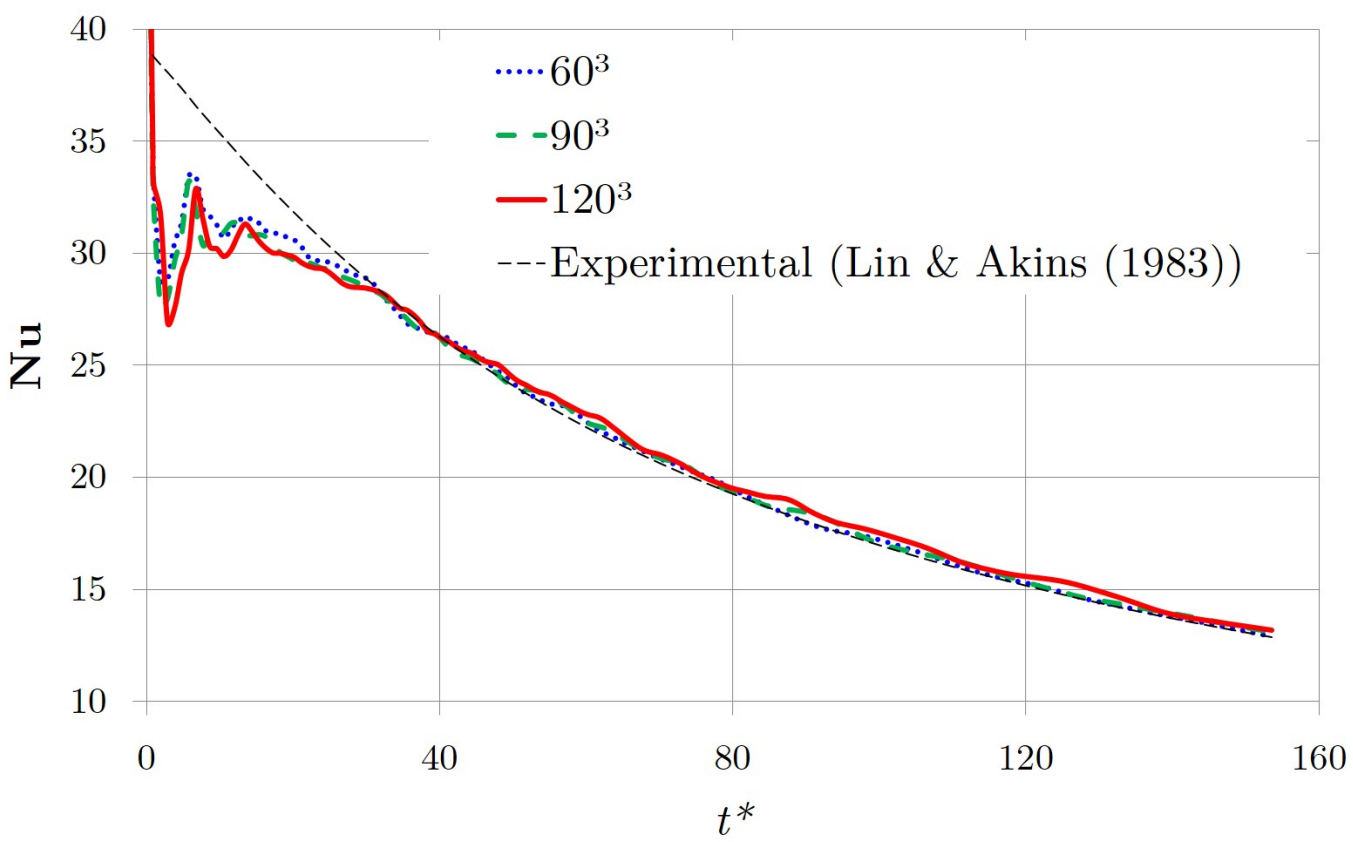

(b) $\mathbf{N u} \times t^{*}$

Figura 4.5: Teste de Malha para Valores de (a) Temperatura e (b) Nusselt variando com o tempo 


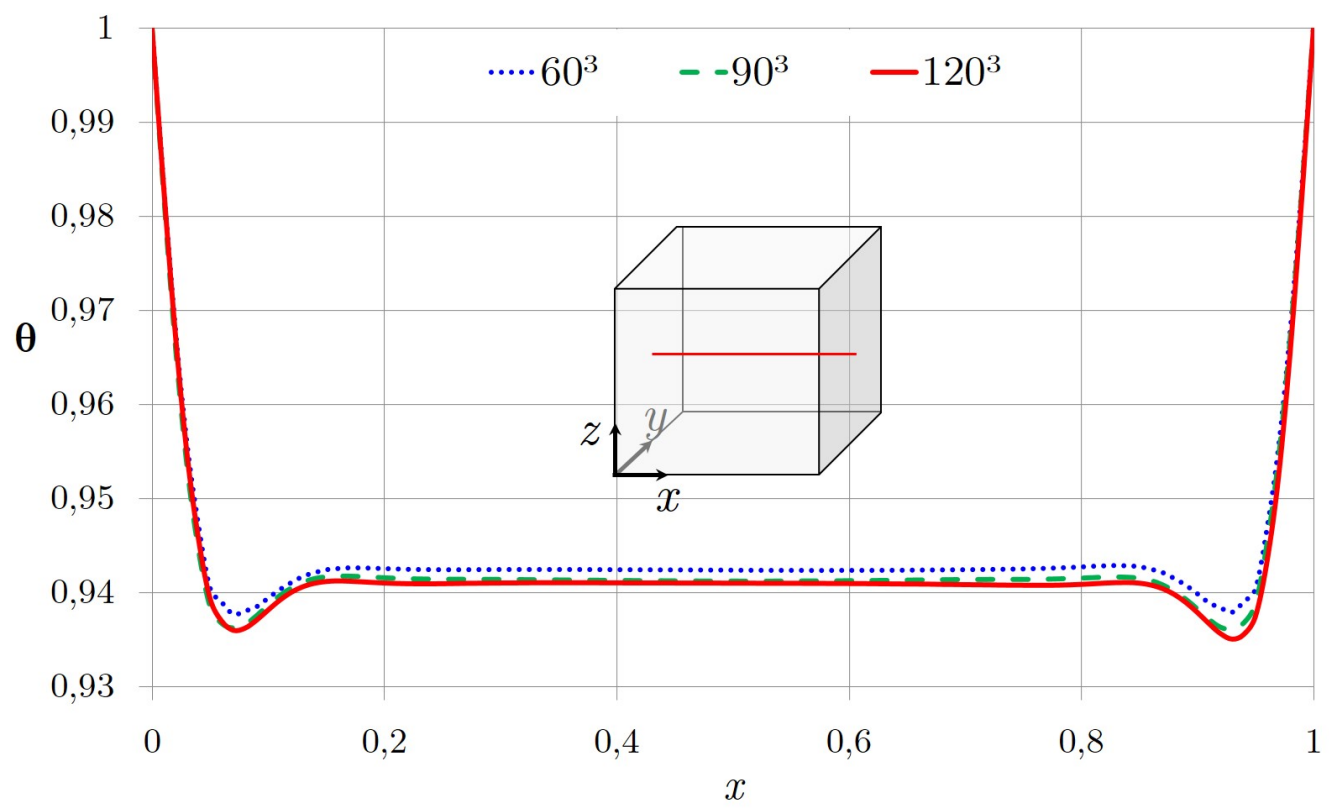

(a) $\theta \times x$

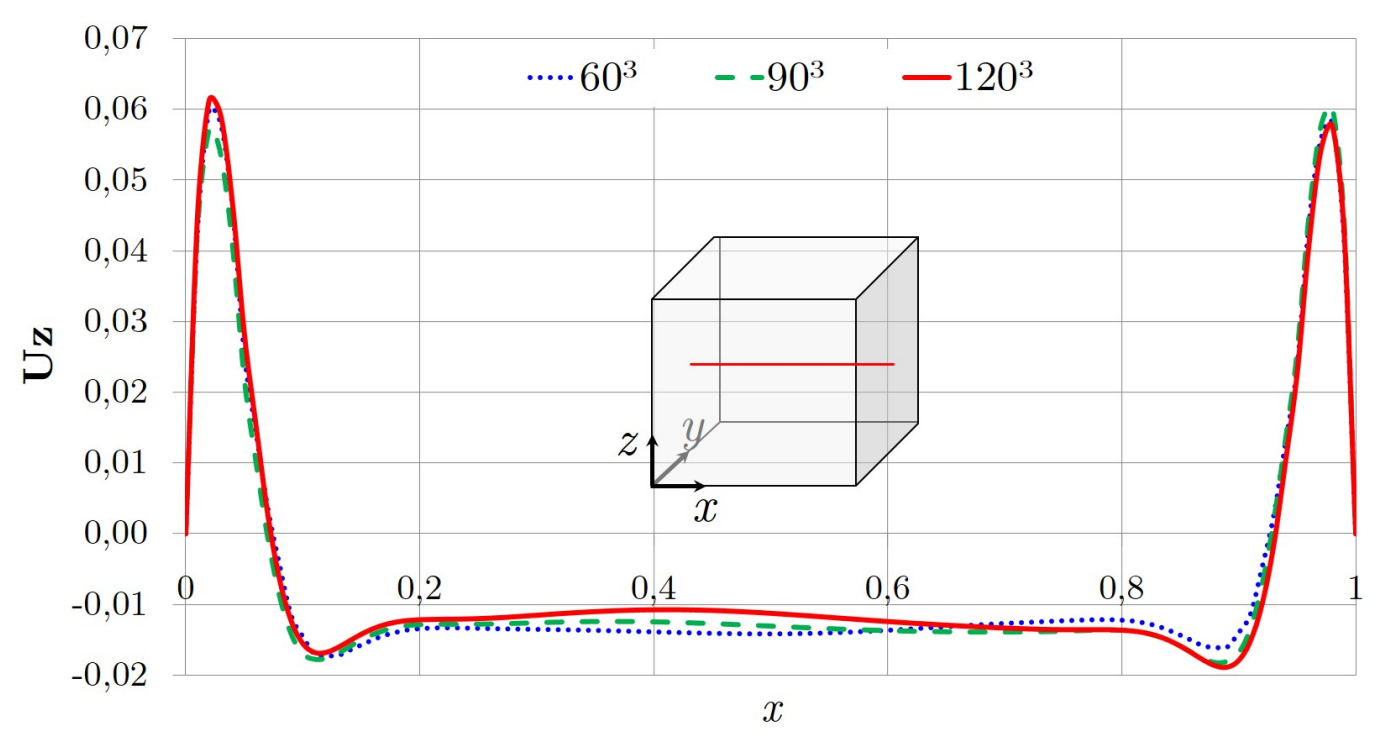

(b) $\mathbf{U} \mathbf{z} \times x$

Figura 4.6: Teste de Malha em $t *=76$ para Valores de (a) Temperatura e (b) Velocidade de camada limite na direção $z$ da linha em $(x, H / 2, H / 2)$

A escolha da configuração de malha se baseou nos resultados mostrados nas Figuras (4.5) e 4.6). Nessas figuras comparamos os valores da temperatura $(\theta \times t)$ variando com o tempo; o valor de Nusselt médio $(\overline{\mathrm{Nu}})$ (Fig. 4.5 varia com o tempo $(\mathrm{Nu} \times t)$ e é comparado com a correlação encontrada por (LIN, 1982), $\mathrm{Nu}=0,6 \mathrm{Ra}^{0,235}$; e valores 
de temperatura $(\theta \times x)$ e velocidade na direção de $z\left(U_{z} \times x\right)$ são comparados de dados extraídos da linha no centro do cubo em $(x, H / 2, H / 2)$. Conforme pode ser observado, não há variação significativa para as três configurações de malha. Apesar da malha com $60^{3}$ elementos ser a ideal, levando-se em consideração o tempo de simulação, optamos pela malha com $90^{3}$ elementos, já que em termos de renderização das superfícies de nível apresentarem um grau de detalhamento maior.

Além da validação da malha, este trabalho realizou a validação do solver utilizado na simulação através da comparação do número de Nusselt versus Rayleight e valores de velocidade encontrados na literatura. Os trabalhos que se encaixaram dentro do aspecto de geometria e condições de contorno foram (LIN; AKINS, 1983) e (TOLLINI, 1996). Mesmo os experimentos terem sido executados com fluidos diferentes da água, como a glicerina e, o tamanho do cubo e variação de temperatura serem diferentes, foi estabelecida uma curva de ajuste entre Nusselt e Rayleigh que serviu como parâmetro de comparação na Fig. 4.7 com a simulação.

No estudo de Lin ( 2 LIN, 1982) e (LIN; AKINS, 1983) $)$ montaram um aparato experimental para analisar os padrões da convecção natural em cavidades cúbicas, com três configurações, medindo 2, 3 e 4 polegadas (respectivamente, 0,0508 m, 0,0762 e 0,1016 $\mathrm{m}$ ) de lado que foram preenchidas por água, etileno glicol ou glicerina. O fluido escolhido para comparação com os dados experimentais foi a água para as três dimensões do cubo, com uma temperatura inicial de $21^{\circ} \mathrm{C}$, variação de temperatura de $\Delta T=0,4$, $\Delta T=1,0$ e $\Delta T=0,5$, o que corresponde, respectivamente a $\mathrm{Ra}=7,93 \times 10^{5}$ (2 pol.), $\mathrm{Ra}=6,98 \times 10^{6}$ (3 pol.) e $\mathrm{Ra}=7,93 \times 10^{6}$ (4 pol.). A Fig. 4.7) apresenta os resultados dessa comparação e a correlação de $\mathrm{Nu}=0,6 \mathrm{Ra}^{0,235}$. Podemos observar que a simulação numérica obteve boa concordância com a curva de ajuste para $\mathrm{Nu} \times \mathrm{Ra}$ em que se obtém o decaimento de Nusselt ao longo do aquecimento da cavidade. 


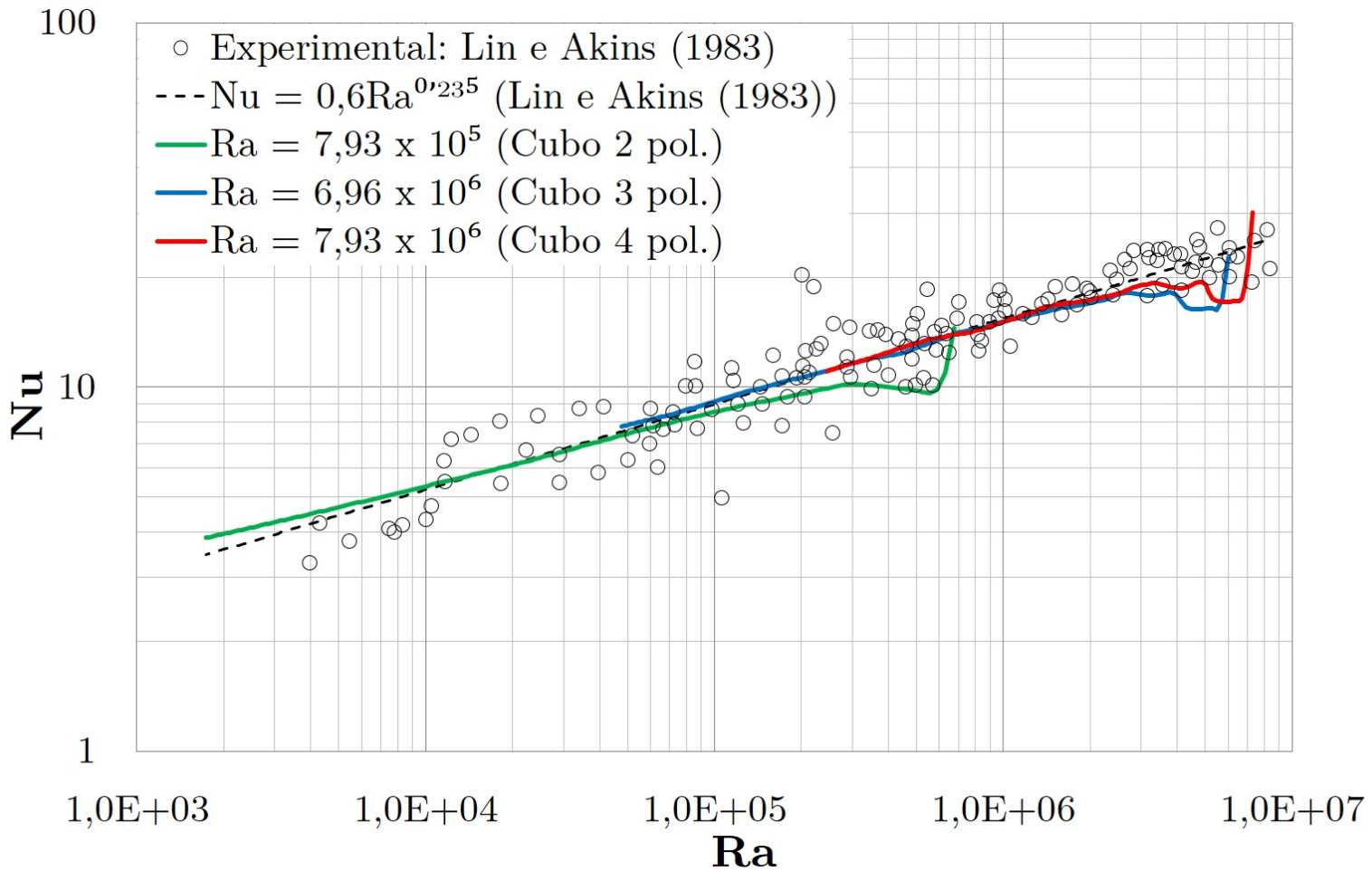

Figura 4.7: Comparação de Nusselt $\times$ Rayleigh do Cubo com dados experimentais ((LIN; AKINS, 1983)).

O outro trabalho que serviu como comparação é o de Tollini ((TOLLINI, 1996)). Neste estudo uma cavidade paralelepípeda retangular de dimensões $H=0,165 \mathrm{~m}, L=0,095$ $\mathrm{m}$ e $W=0,065 \mathrm{~m}$ (caixa Tetra Park original) foi construída e em seu interior preenchida por água. Três situações de experimentos foram comparadas com a simulação: a primeira com a temperatura inicial de $21^{\circ} \mathrm{C}$ e variação de temperatura de $\Delta T=14$, a segunda e terceira com a temperatura inicial de $20{ }^{\circ} \mathrm{C}$ e variação de temperatura de $\Delta T=25$ e $\Delta T=45$, correspondendo, respectivamente a $\mathrm{Ra}=9,48 \times 10^{8}$, $\mathrm{Ra}=$ $1,69 \times 10^{9}$ e $\mathrm{Ra}=3,05 \times 10^{9}$. Na Fig. 4.8 temos o resultado da comparação e a apresentação da mesma correlação de $\mathrm{Nu}=0,2493 \mathrm{Ra}^{0,2751}$ em que obtivemos uma boa concordância com a simulação numérica. 


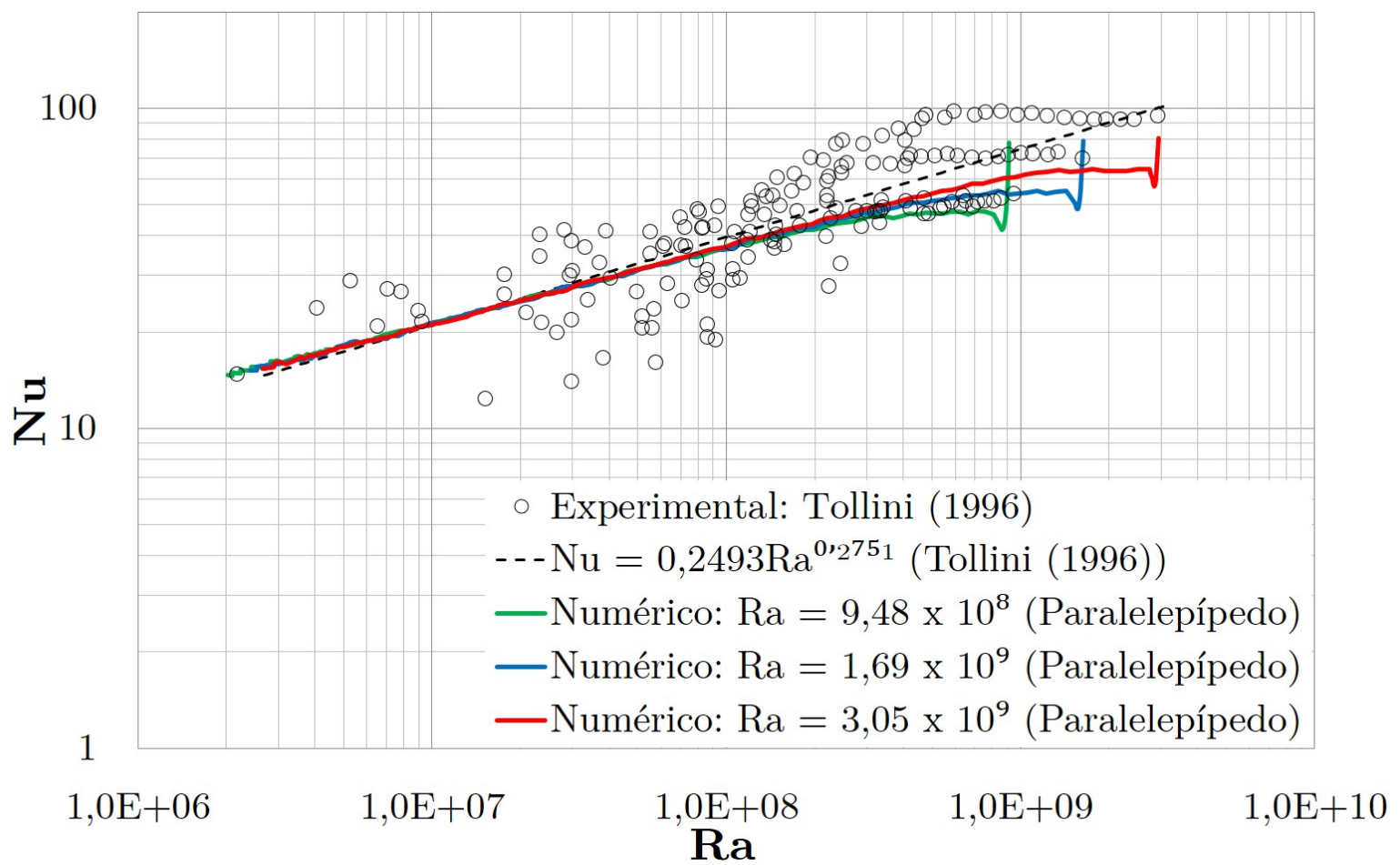

Figura 4.8: Comparação de Nusselt $\times$ Rayleigh do Cubo com dados experimentais ((TOLLINI, 1996)).

Os dados de velocidade são apresentados apenas no trabalho de (LIN; AKINS, 1983) e estão mostrados na Fig. 4.9. Os dados foram coletados e medidos do centro do cubo até a parede lateral vertical, na direção $y$. Os valores da distância foram adimensionalizados pela altura $((y-(H / 2)) /(H / 2))$ e a velocidade pela velocidade de referência $U_{0}$, definida na Eq. 2.5.1 $\left(u_{z} / U_{0}\right)$. Uma complementação aos resultados apresentados na Fig. (4.9) são mostrados nas Fig. 4.10 a 4.12. Em ambas as imagens, pode-se destacar a intensidade da velocidade próximo a parede até a metade superior da seção de visualização $(y=H / 2)$. Para os valores coletados da metade inferior da seção, observa-se a ascensão de fluido no centro do cubo. 


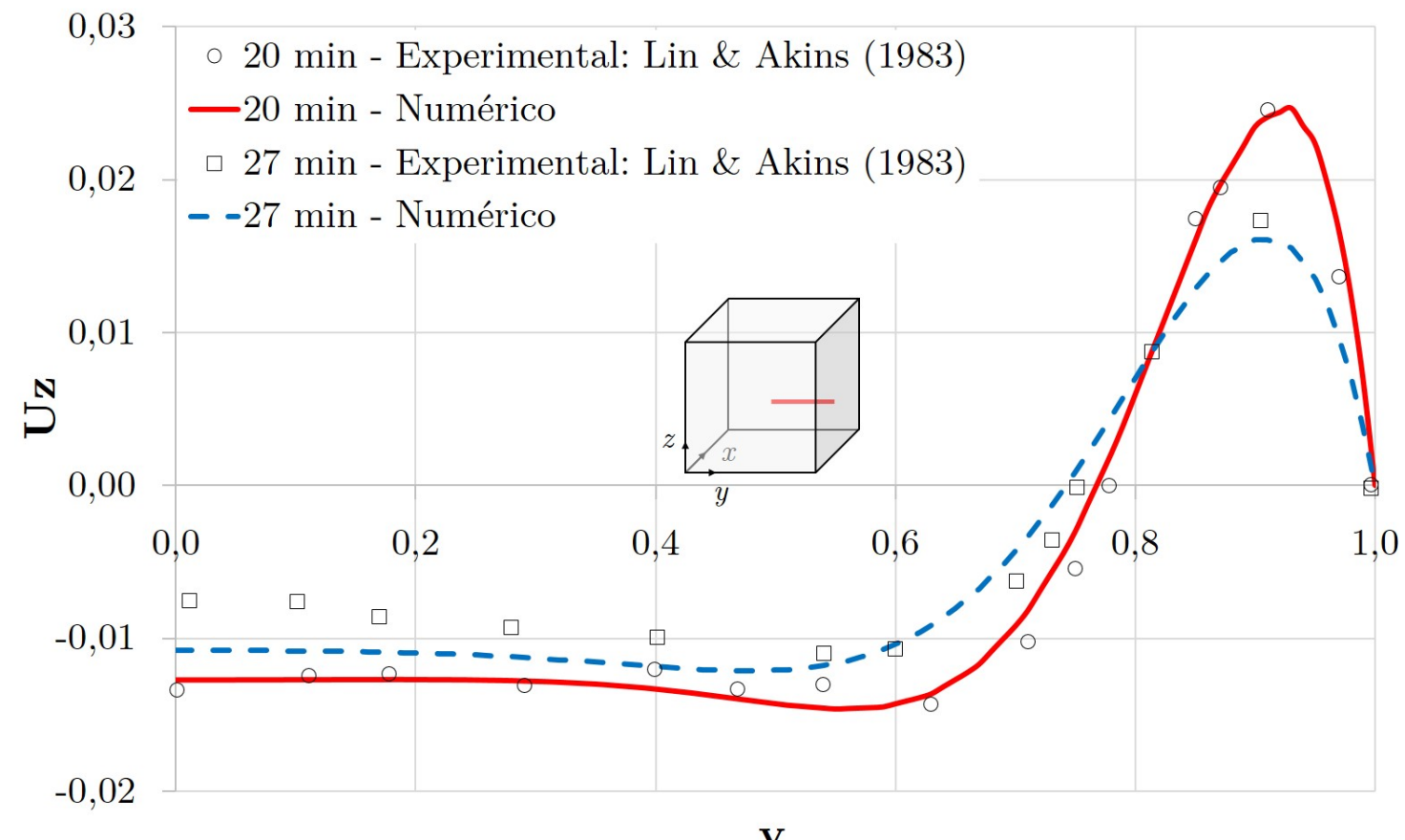

Figura 4.9: Comparação da Velocidade $U_{z}$ com dados experimentais para o Cubo de 3 pol. (com Etileno Glicol) apresentado em (LIN; AKINS, 1983).

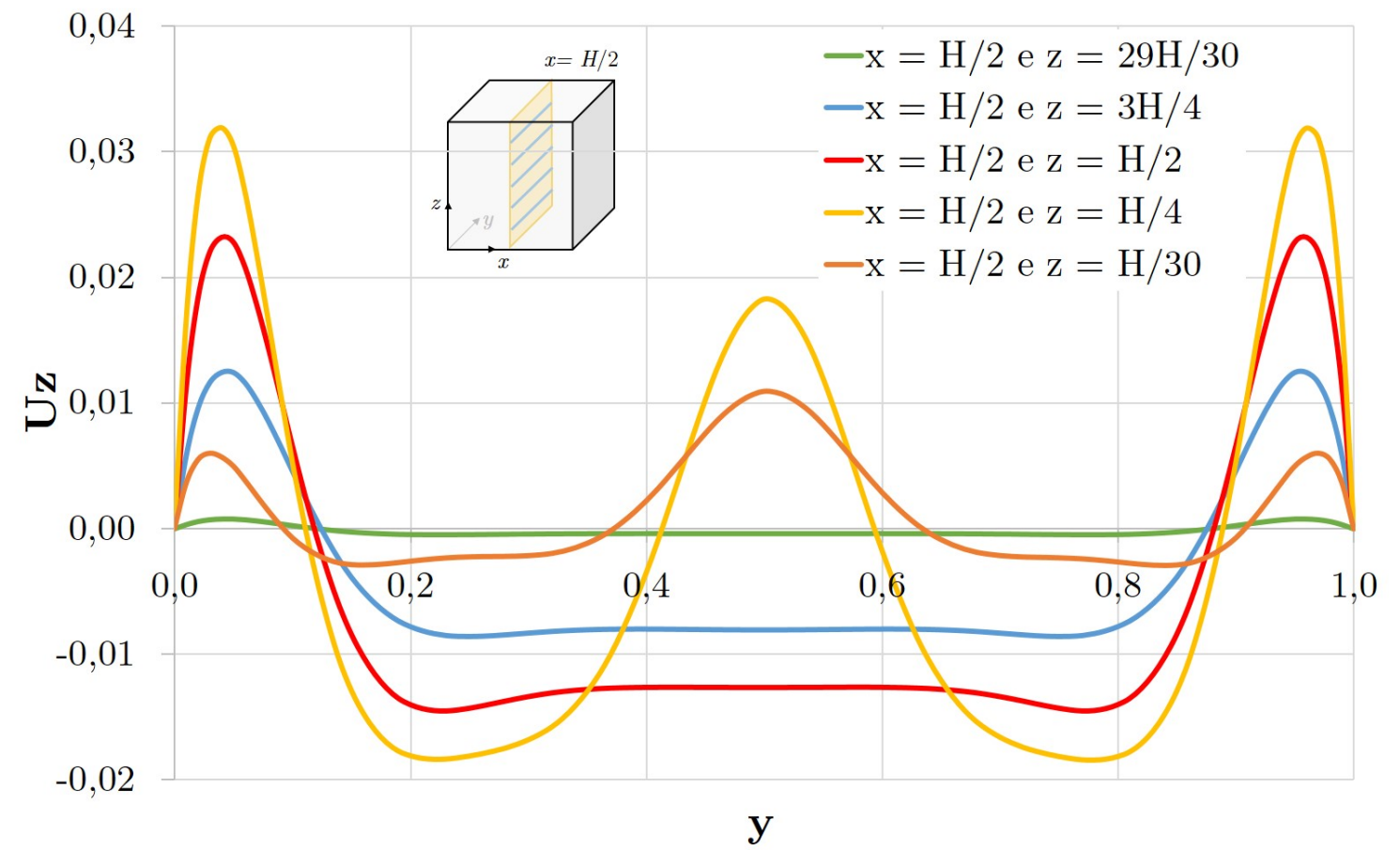

Figura 4.10: Perfis de Velocidade sobre o plano $y=H / 2$ na direção $x$ com a variação da altura (z) para o Cubo de 3 pol. (com Etileno Glicol) apresentado em (LIN; AKINS, 1983). 


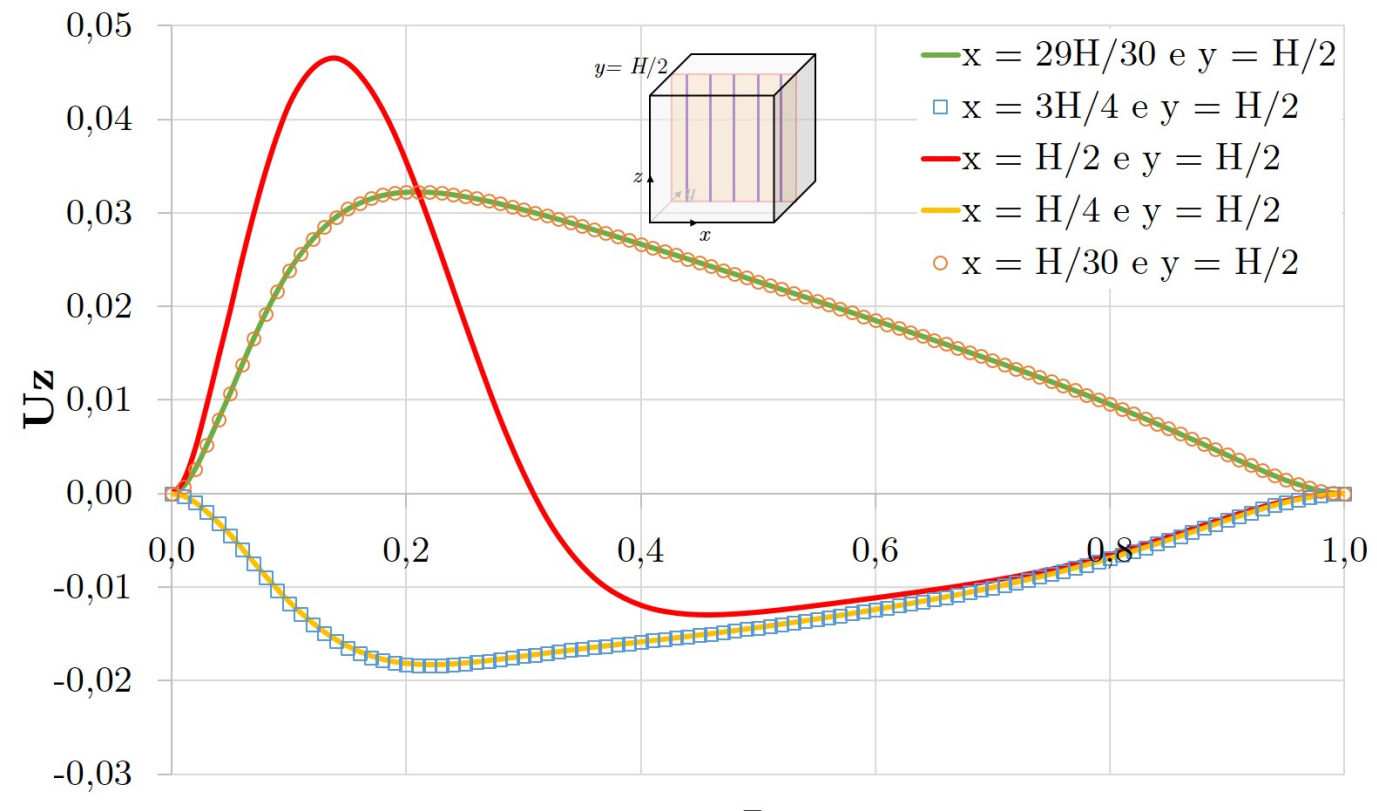

Figura 4.11: Perfis de Velocidade sobre o plano $y=H / 2$ na direção $x$ com a variação da altura (z) para o Cubo de 3 pol. (com Etileno Glicol) apresentado em (LIN; AKINS, 1983).

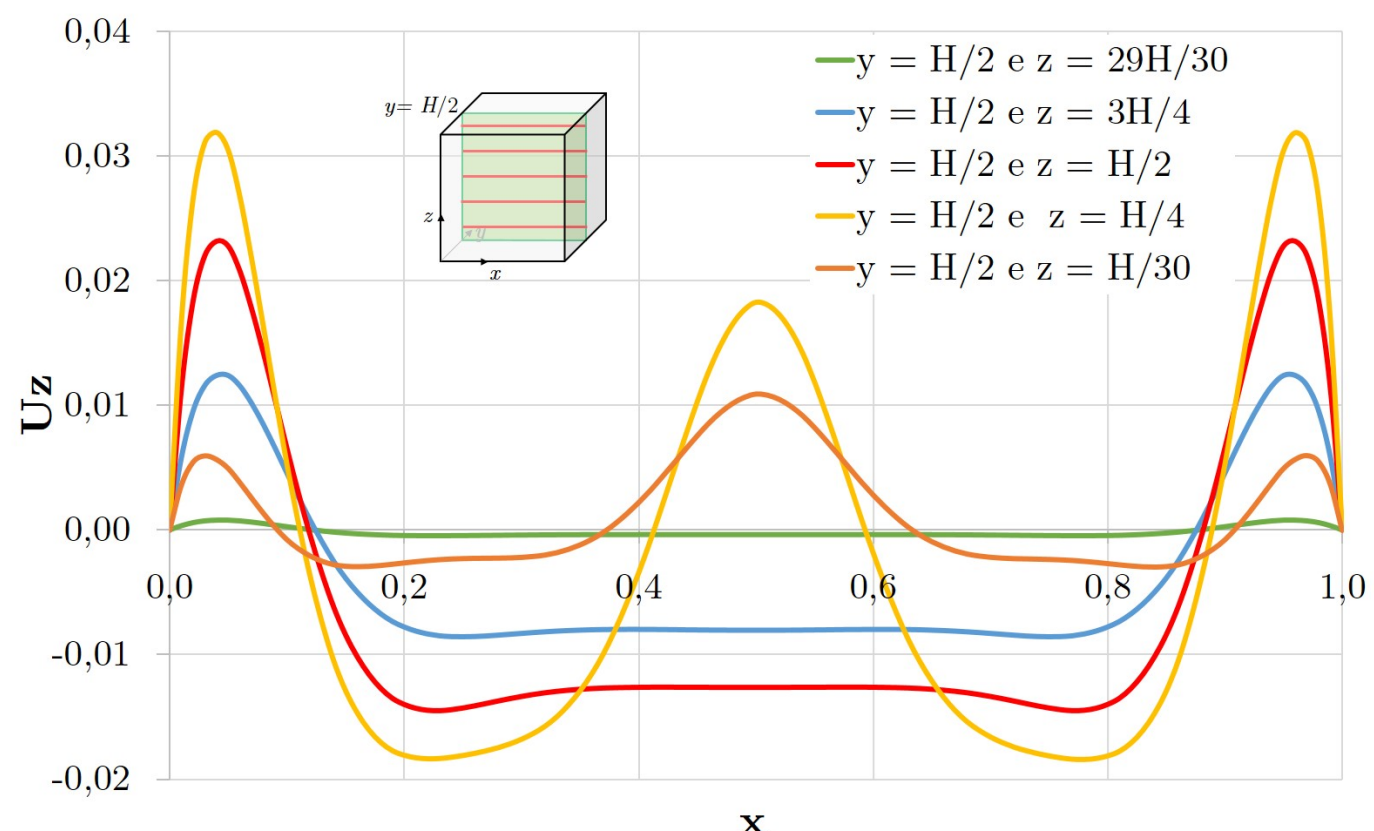

Figura 4.12: Perfis de Velocidade sobre o plano $y=H / 2$ na direção $x$ com a variação da altura ( $z$ ) para o Cubo de 3 pol. (com Etileno Glicol) apresentado em (LIN; AKINS, 1983). 


\subsection{Caracterização da Convecção Natural na Cavidade}

A convecção natural na cavidade, em que todas as faces são aquecidas simultaneamente à mesma temperatura, é caracterizada pela formação inicial de uma camada limite vertical, em que o fluido sobe até a parede superior e desce, formando duas grandes células de circulação em uma região de estratificação da temperatura. Na sequência, a parede inferior aquecida inicia o desprendimento de bolhas de fluido no formato de cogumelos que se expandem até encontrarem a camada de fluido descendente. Esse processo se repete e diminui à medida que o fluido no interior se aproxima da temperatura de aquecimento das paredes. Uma ilustração dessas regiões está esboçada na Fig. (1.3) e mostrada abaixo.

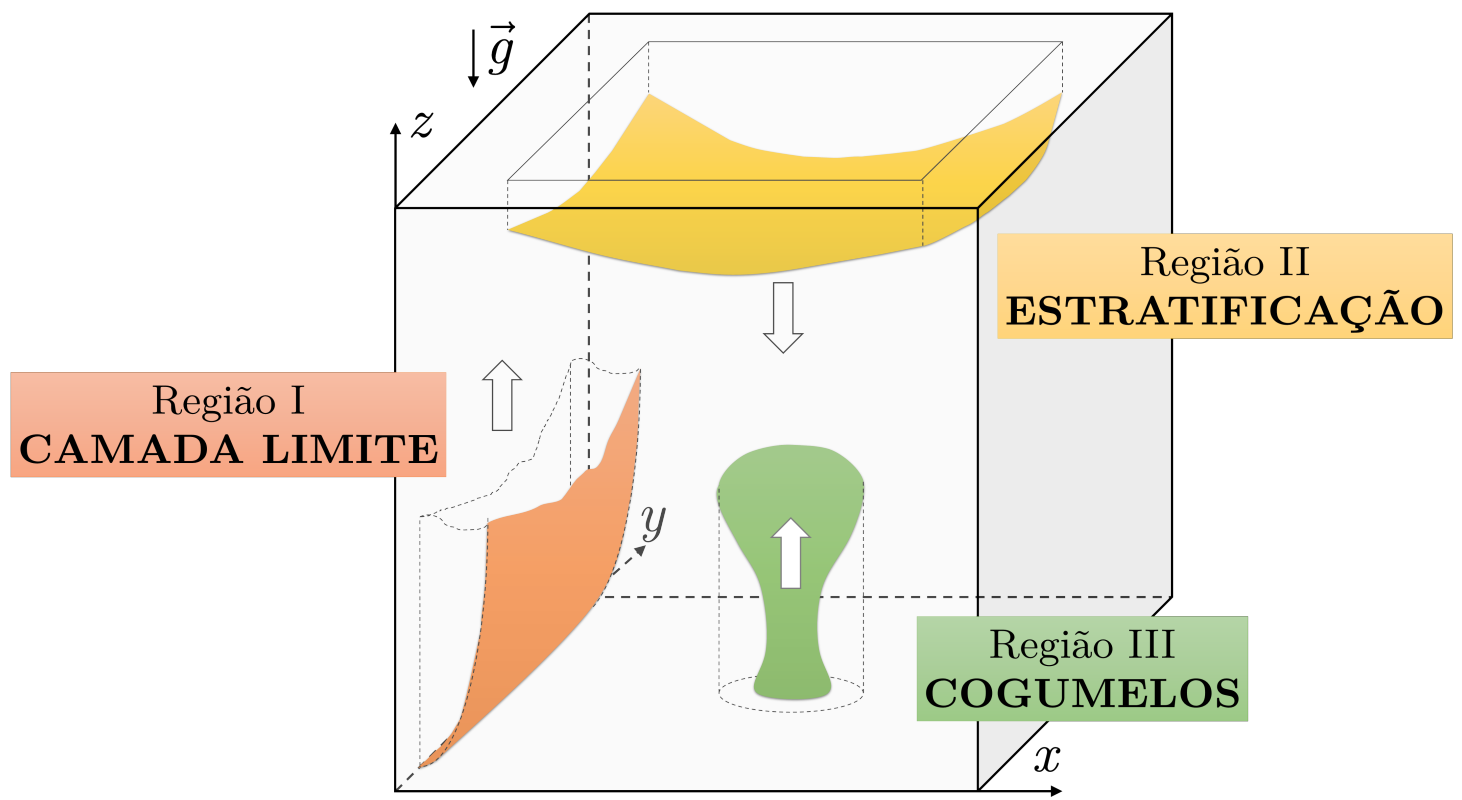

Características da Convecção Natural na Cavidade: Escoamento.

Para se analisar as características do escoamento, mostradas na Fig. (1.3), optou-se por realizar a simulação do cubo (lado medindo 4 pol. ou 0,1016 m) e do paralelepípedo retangular $(H=0,165 \mathrm{~m}, L=0,095 \mathrm{~m}$ e $W=0,065 \mathrm{~m})$. Para isso, foram utilizados os seguintes parâmetros na simulação realizada no OpenFOAM: Temperatura inicial $T_{0}=21{ }^{\circ} \mathrm{C}$, temperatura de aquecimento das paredes $T_{W}=21,25{ }^{\circ} \mathrm{C}$ que se traduz em $\mathrm{Ra}=4 \times 10^{6}$ para o cubo e $\mathrm{Ra}=2 \times 10^{7}$ para o paralelepípedo retangular (Tabela 4.3 ). 


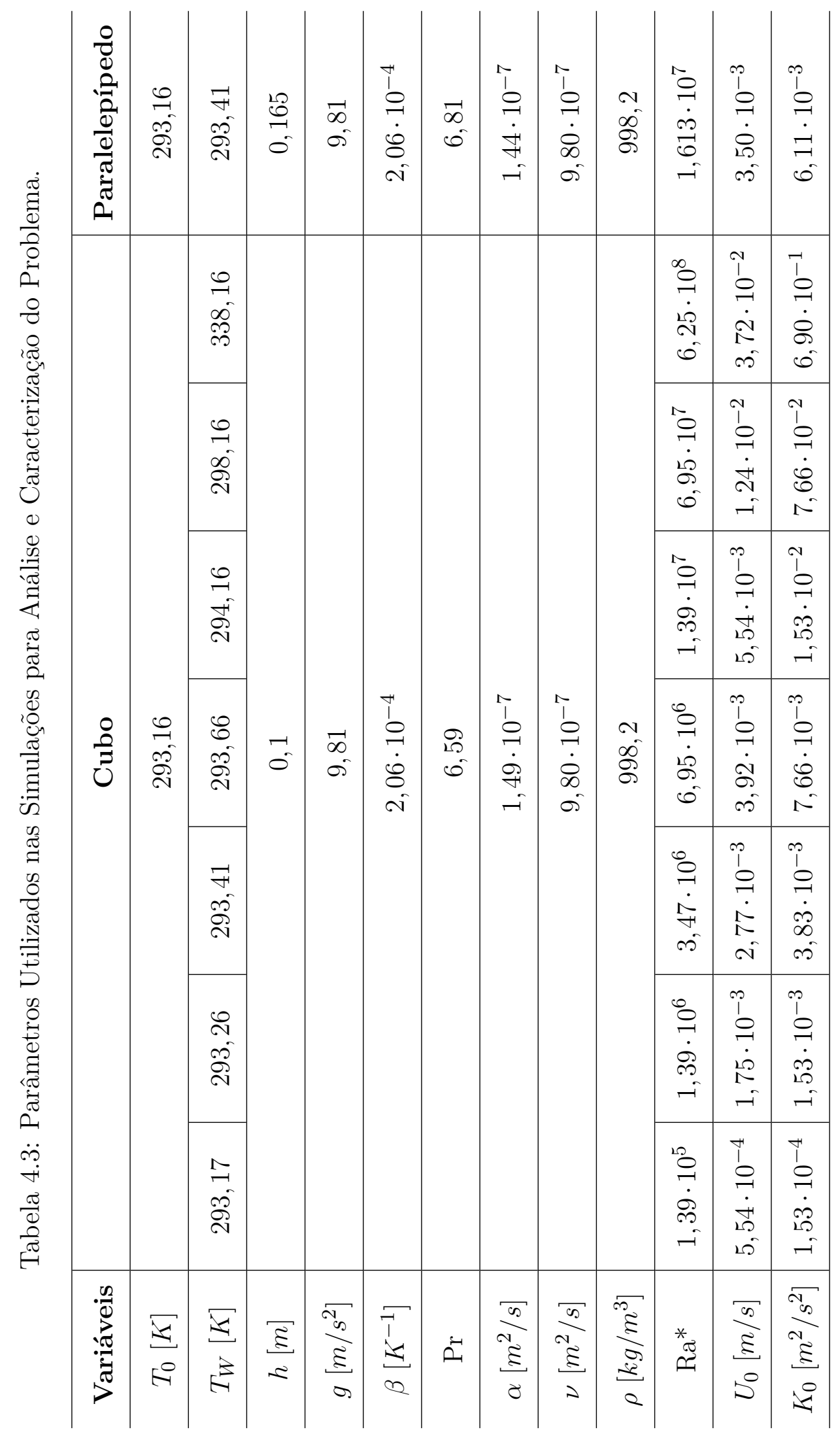


A evolução do campo de temperatura, de acordo com a variação do número de Rayleigh global, esboçada na Fig. (4.18), foi elaborada através de várias observações de simulações selecionadas e distribuídas com os seguintes números de Rayleigh: $\mathrm{Ra}=2 \times 10^{5}$ $(\Delta T=0,01), \quad \mathrm{Ra}=2 \times 10^{6}(\Delta T=0,1), \mathrm{Ra}=4 \times 10^{6}(\Delta T=0,25), \quad \mathrm{Ra}=2 \times 10^{7}$ $(\Delta T=1), \mathrm{Ra}=8 \times 10^{7}(\Delta T=5), \mathrm{Ra}=7 \times 10^{8}(\Delta T=45)$.

Quando se começou a observar o campo de temperatura verificamos que a região mais fria está sempre na parte inferior da cavidade, acentuando-se esta característica nas camadas de fluido mais próximas às paredes verticais, caracterizando uma estratificação térmica na cavidade, como pode ser observado nos "Contornos de T" nas Figs. 4.14, 4.15. 4.16 e 4.17). Para as camadas mais centrais, a estratificação da temperatura, ao longo da linha vertical da cavidade, não é tão acentuada. Isso se explica pelo fato de que, nas camadas centrais da cavidade, as velocidades são relativamente baixas, conforme observado nos "Contornos de u" nas Figs. 4.14, 4.15, 4.16 e 4.17). Por outro lado, as correntes convectivas explicam a localização da região mais fria na parte inferior da cavidade.

No que diz respeito ao campo de velocidade é interessante observar que, conforme pode ser visto nas ilustrações dos vetores de velocidade e de vorticidade das Figs. 4.14 , 4.15, 4.16 e 4.17). Para as camadas mais centrais, a estratificação da temperatura, ao longo da linha vertical da cavidade, não é tão acentuada. Isso se explica pelo fato de que, nas camadas centrais da cavidade, as velocidades são relativamente baixas, conforme observado nos "Contornos de u" nas Figs. 4.14, 4.15, 4.16 e 4.17), o centro de circulação do movimento principal se situa muito próximo à parede, e que algumas partículas que sobem não chegam a atingir a camada aquecida superior, desviando-se de seus caminhos e fazendo uma circulação menor, tomando um movimento descendente em direção à face inferior.

O movimento do fluido dentro da cavidade representa a composição dos efeitos da transferência de calor através de cada uma das suas seis faces, já que o fluido se encontrava em repouso e em equilíbrio térmico com o meio exterior. A face inferior, a superior e as quatro faces laterais, representam, separadamente, um diferente efeito sobre o movimento do fluido.

As quatro faces laterais verticais, ao receberem calor, ocasionam uma tendência para o fluido subir rapidamente através de uma fina camada junto às paredes laterais e descer lentamente por uma grande massa na região central da cavidade. 
Como o somatório das áreas das quatro faces laterais representa $2 / 3$ da área externa da cavidade cúbica ou 4/5 da cavidade paralelepípeda e, com a influência da aceleração da gravidade, é de se supor que o movimento ocasionado pela transferência de calor nelas seja de importância primordial para o movimento do fluido dentro da cavidade.

A face inferior, por sua vez, ocasiona uma tendência para o fluido subir como um todo, especialmente pelo centro da cavidade. Como há uma grande massa de fluido descendo pelo centro, impulsionada pelo movimento principal do fluido, alguns padrões de recirculação são criados na base da cavidade, análogos a escoamentos do tipo Células de Bénard, mostrado nas isosuperfícies de temperatura das Figs. 4.15 e 4.17). De fato essas estruturas assemelham-se a pequenas térmicas ascendentes, em formato de cogumelo, como relatado por (SPARROW; HUSAR; GOLDSTEIN, 1970) na Fig. (1.4).

Já a face superior da cavidade causa a formação de uma pequena camada aquecida e mais leve próxima do topo, onde as velocidades são relativamente muito baixas. O fluido que sobe pelas paredes laterais, ao atingir a camada aquecida junto ao topo, se dirige em direção ao centro da cavidade, iniciando um movimento lento de descida. Desta forma, é de se supor que a influência da face superior no movimento do fluido dentro da cavidade seja quase nula, ilustrado pelos vetores das Figs. (4.14 e 4.16).

Ao longo das simulações realizadas, durante o período restante, o movimento do fluido não se altera muito, havendo apenas um pequeno aumento da espessura da camada superior, caracterizada por baixas velocidades, fazendo, consequentemente, com que as partículas que atingem o topo não tenham energia para ir até o centro antes de iniciar o movimento descendente. A velocidade média do fluido começam a diminuir gradualmente e a camada estável no topo cresceu um pouco. Além disso, as partículas de células de Bénard parecem ter diminuído de velocidade, especialmente pela diminuição aparente do tamanho das células, até que a temperatura em toda a cavidade torne-se $T_{W}$ e o escoamento cesse. 


\section{Resultados}

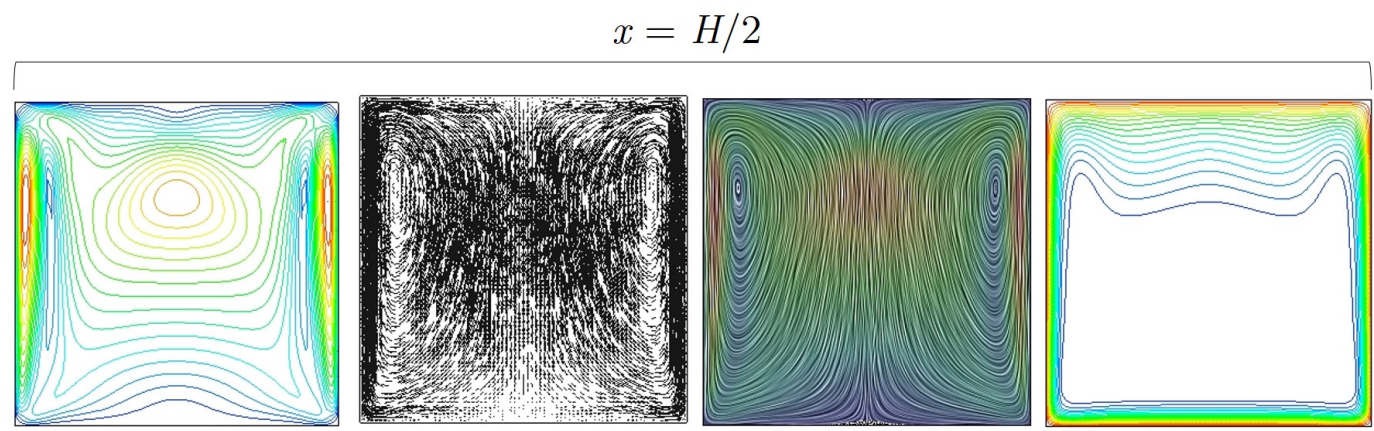

(a) $t=80 \mathrm{~s}$
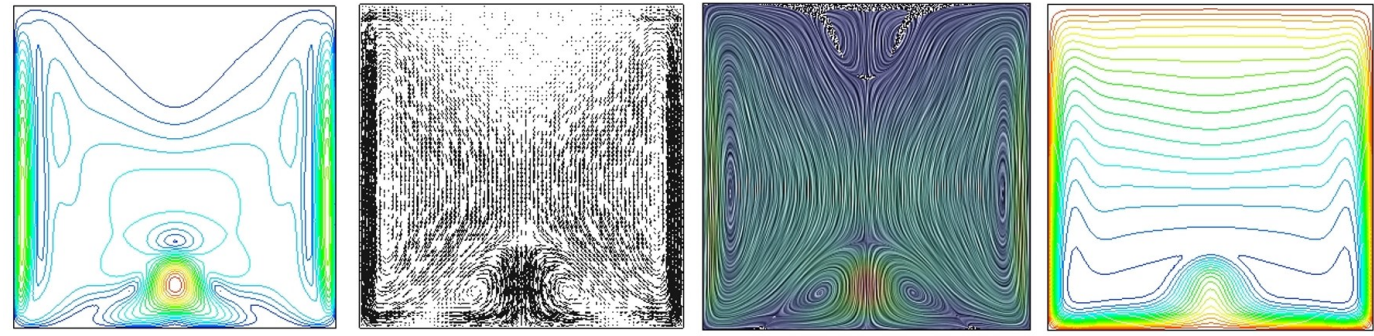

(b) $t=210 \mathrm{~s}$
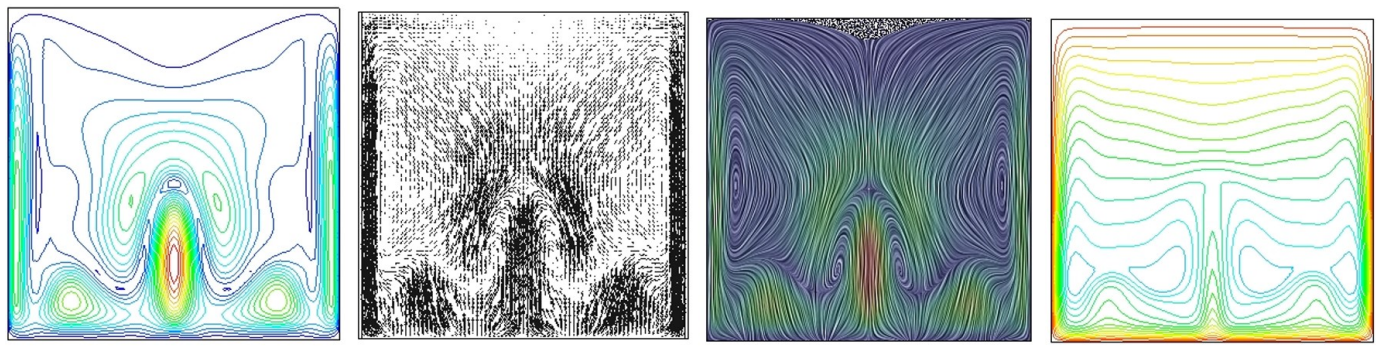

(c) $t=310 \mathrm{~s}$
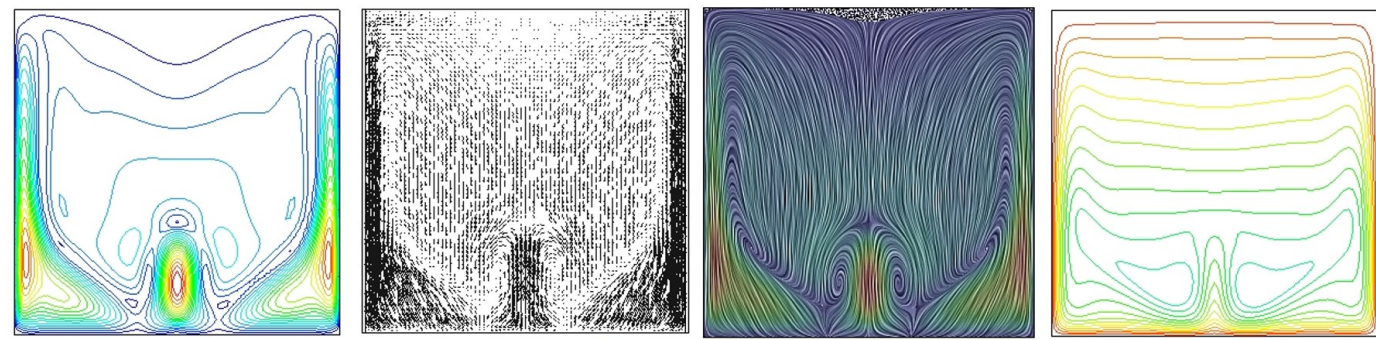

(d) $t=430 \mathrm{~s}$
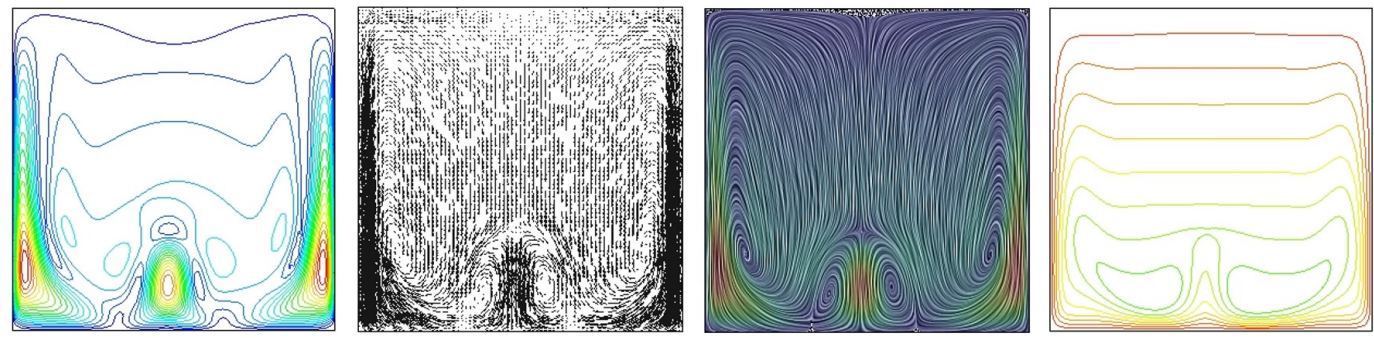

(e) $t=670 \mathrm{~s}$

Contornos de $\mathbf{u} \quad$ Vetores de $\mathbf{u}$ Linhas de corrente Contornos de $T$

Figura 4.14: Visualização das Características do Cubo em $x=H / 2$ para Ra $=4 \times 10^{6}$ 


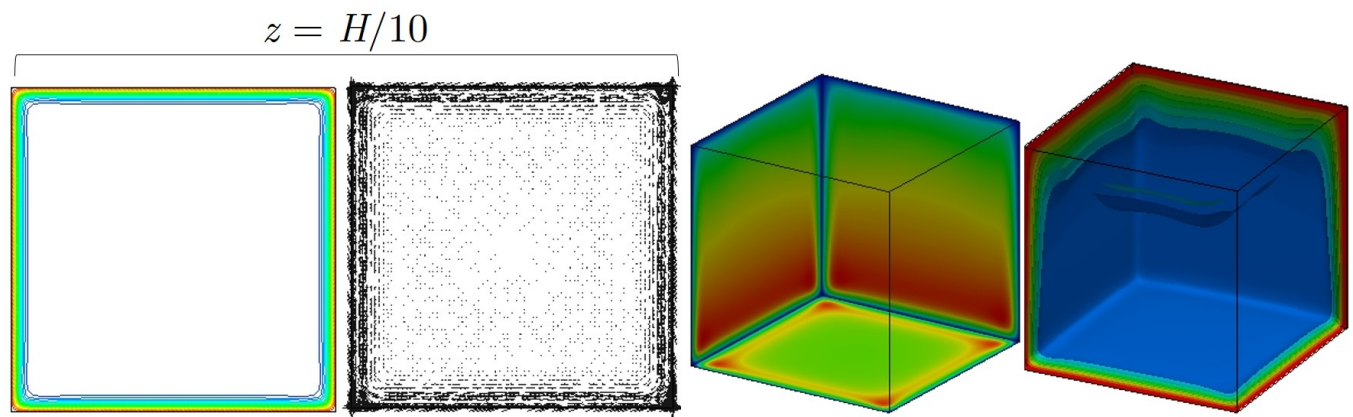

(a) $t=80 \mathrm{~s}$
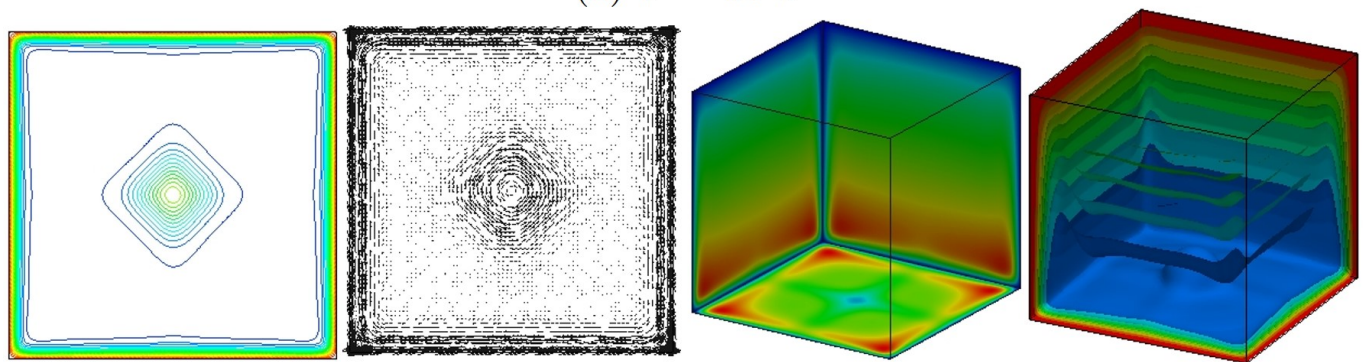

(b) $t=210 \mathrm{~s}$
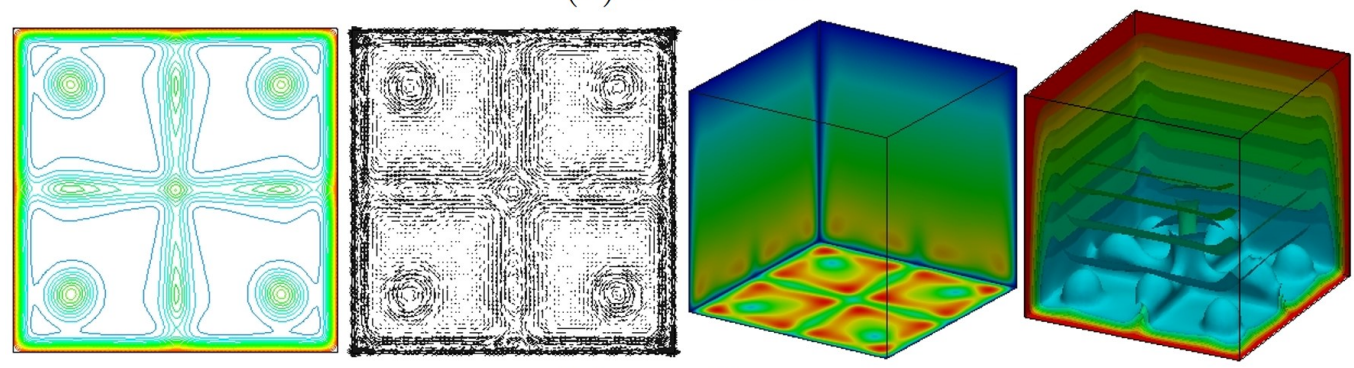

(c) $t=310 \mathrm{~s}$
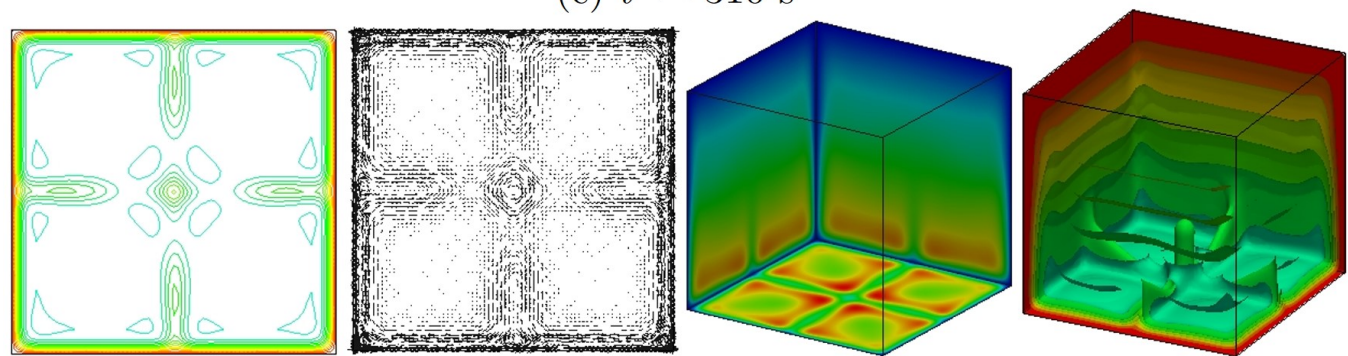

(d) $t=430 \mathrm{~s}$
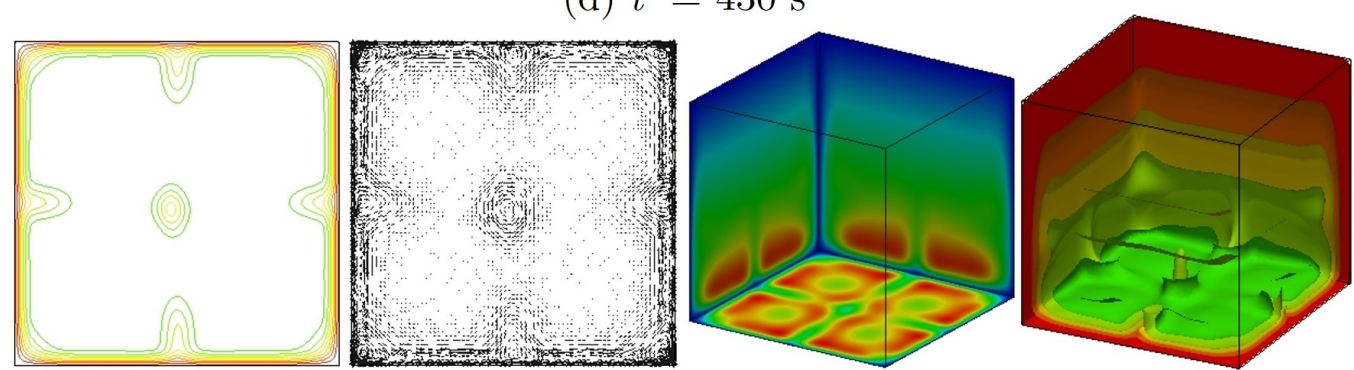

(e) $t=670 \mathrm{~s}$

Contornos de $T$ Vetores vorticidade Nusselt Isosuperfícies de $T$

Figura 4.15: Visualização das Características do Cubo em $y=H / 10$ para $\mathrm{Ra}=4 \times 10^{6}$ 
$x=L / 2$
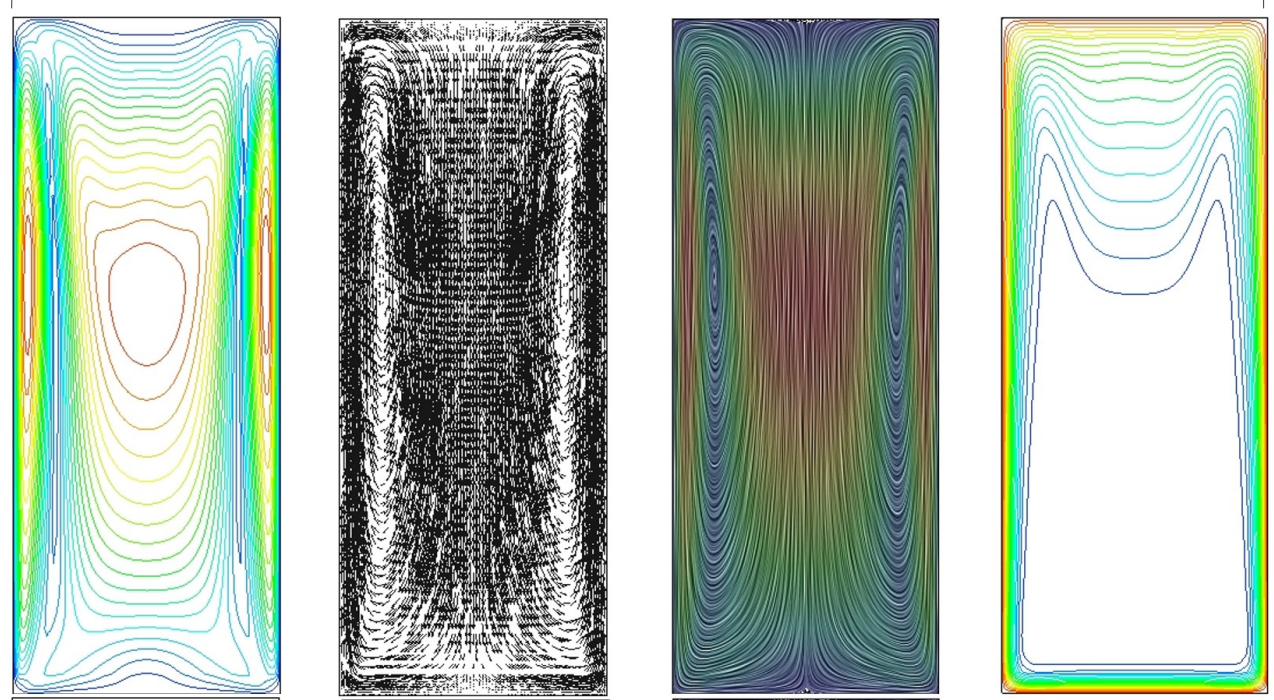

$\infty$
8
11
$+\infty$
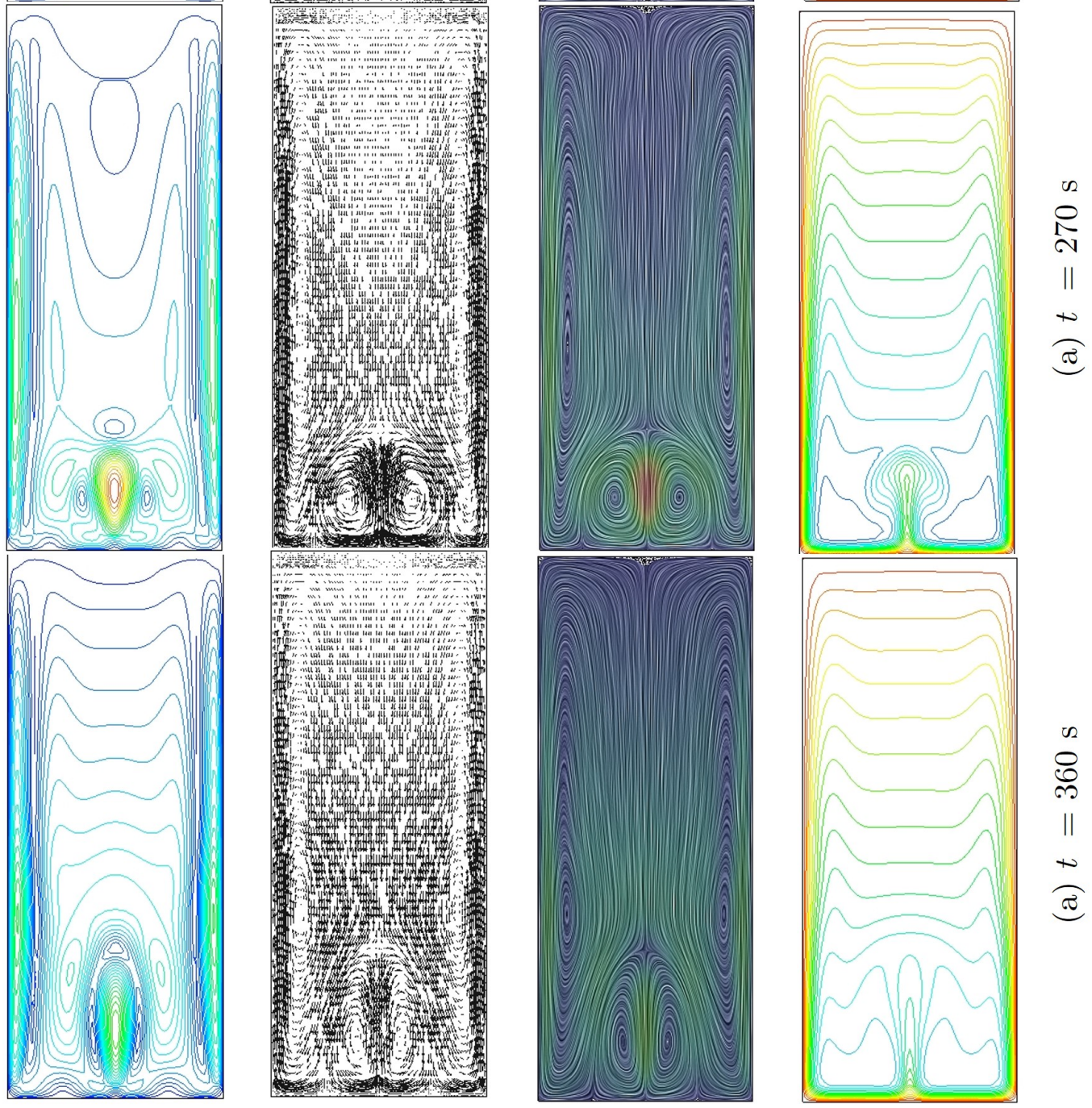

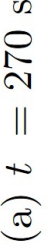

Contornos de $\mathbf{u}$

Vetores de $\mathbf{u}$ Linhas de corrente Contornos de $T$

Figura 4.16: Visualização das Características do Paralelepípedo Retangular em $x=$ $H / 2$ para $\mathrm{Ra}=4 \times 10^{6}$ 


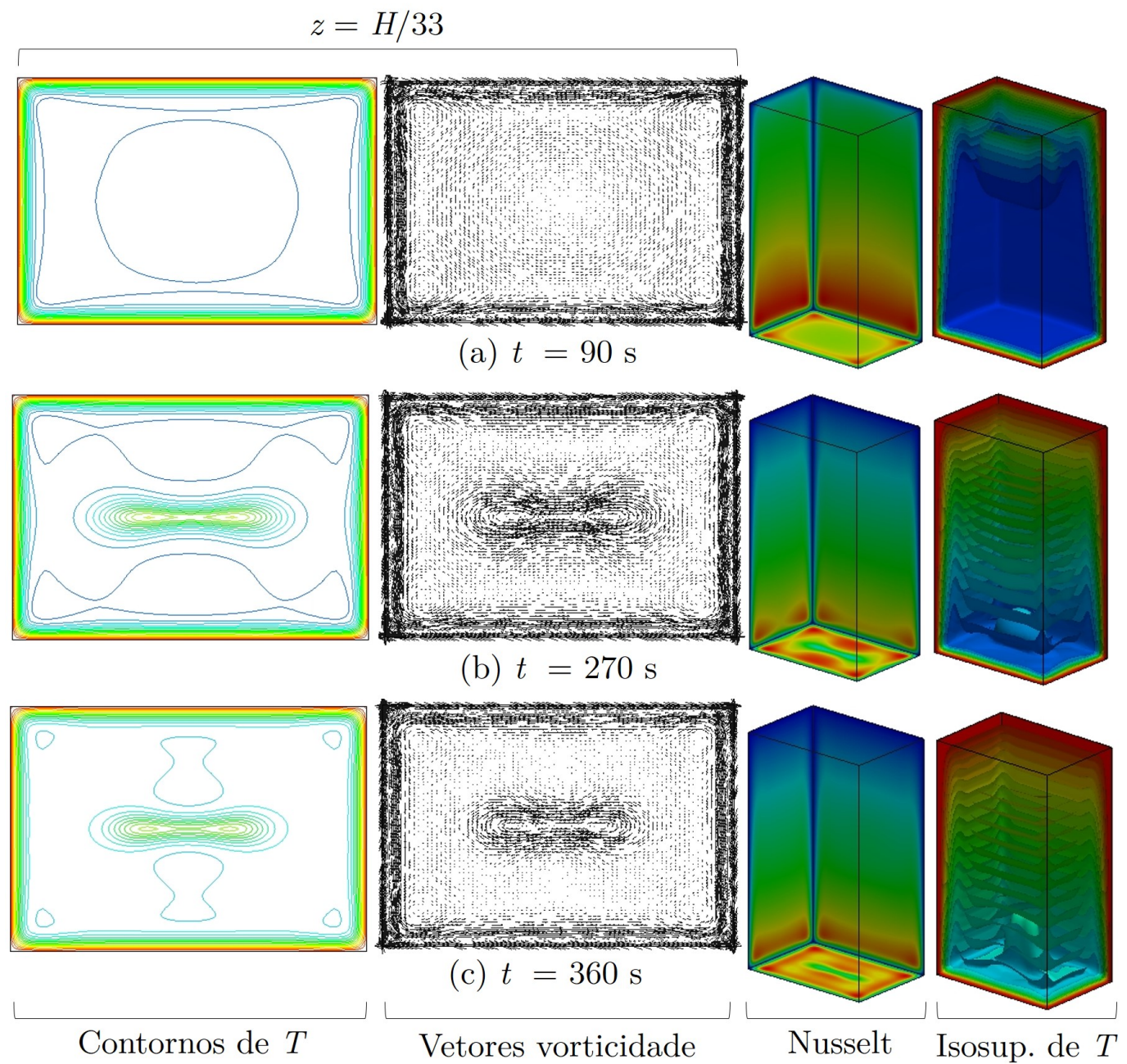

Figura 4.17: Visualização das Características do Paralelepípedo Retangular em $y=$ $H / 10$ para $\mathrm{Ra}=4 \times 10^{6}$

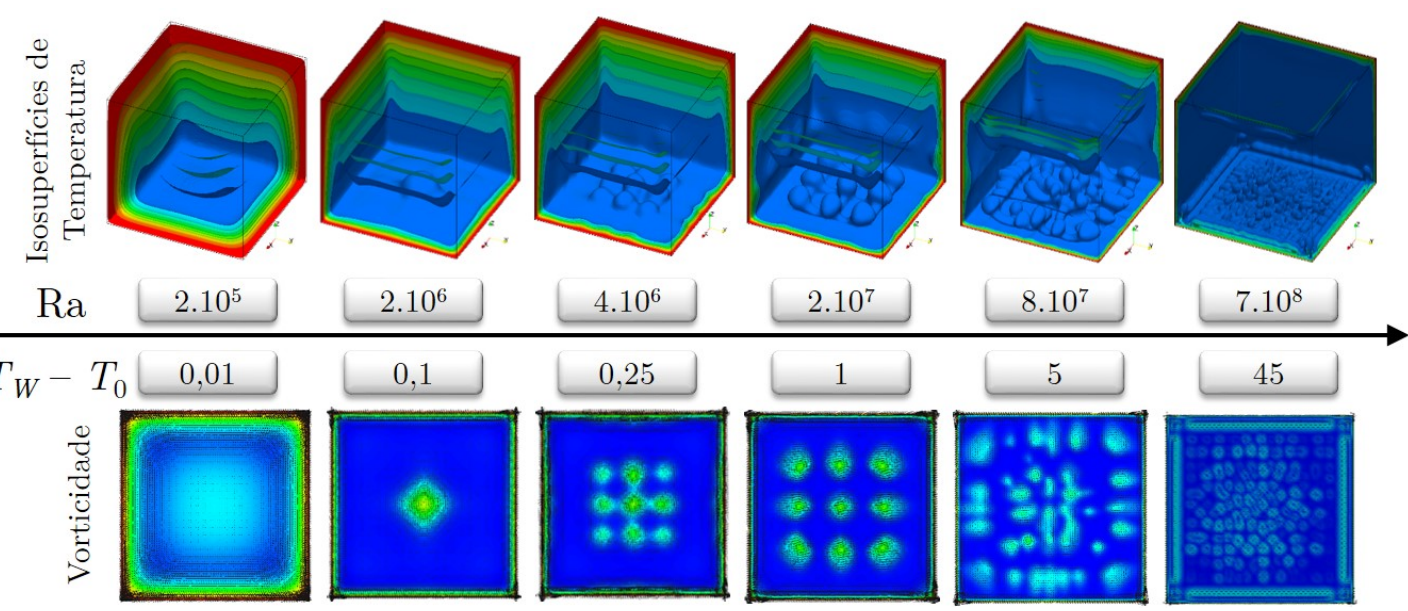

Figura 4.18: Características da Convecção Natural na Cavidade. 


\subsubsection{Relação entre Energia Cinética e o Surgimento dos "Cogumelos"}

Os primeiros 5 min de tempo físico de simulação são os que mais apresentam os efeitos da transferência de calor. A energia cinética atinge seu pico neste período, mostrado nas Figs. 4.19 e 4.20). O primeiro pico de energia cinética tem relação com o aquecimento inicial da parede e que faz com que um parcela do fluido suba com velocidade superior à parcela de fluido da parede inferior.

Na sequência, os picos secundários de energia cinética tem relação direta com o surgimento das bolhas de fluidos aquecidos (cogumelos). As Figs. 4.21 e 4.22 mostram o exato instante em que os padrões dessas bolhas se desenvolvem, respectivamente, para o cubo e o paralelepípedo retangular. Essas estruturas no cubo, para o caso de $\mathrm{Ra}=3,52 \times 10^{6}$, surgem inicialmente como uma única bolha, que no próximo ciclo se revela na forma de uma matriz $3 \times 3$, diminuindo sua formação até que a energia cinética cesse e todo o fluido se aqueça igualmente. Para a situação analisada do paralelepípedo retangular com $\mathrm{Ra}=1,58 \times 10^{7}$ uma única bolha se forma e do mesmo modo reaparece nos ciclos seguintes, até que cesse o processo de convecção natural.

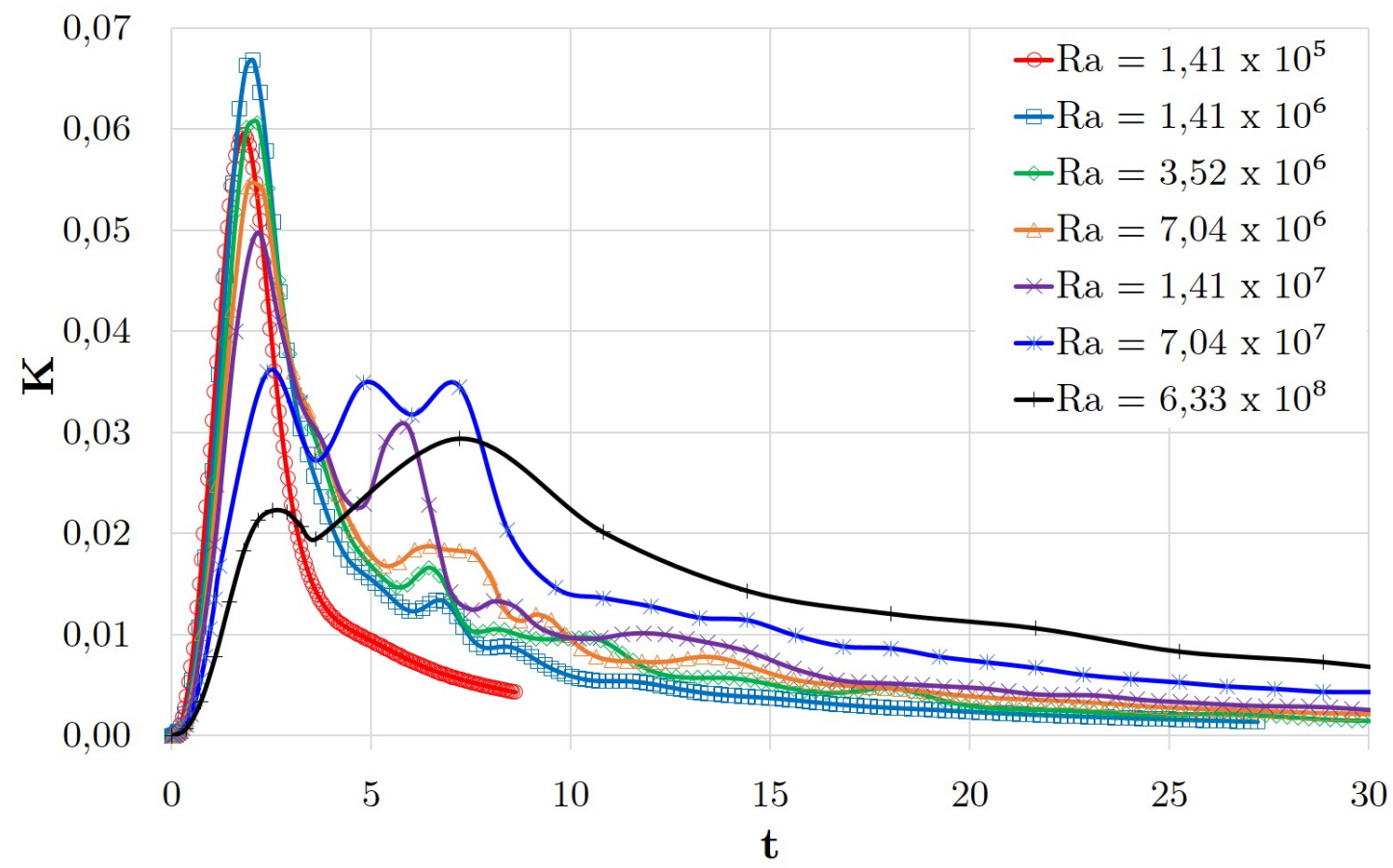

Figura 4.19: Variação da Energia Cinética no Cubo para Diferentes $T_{W}$. 


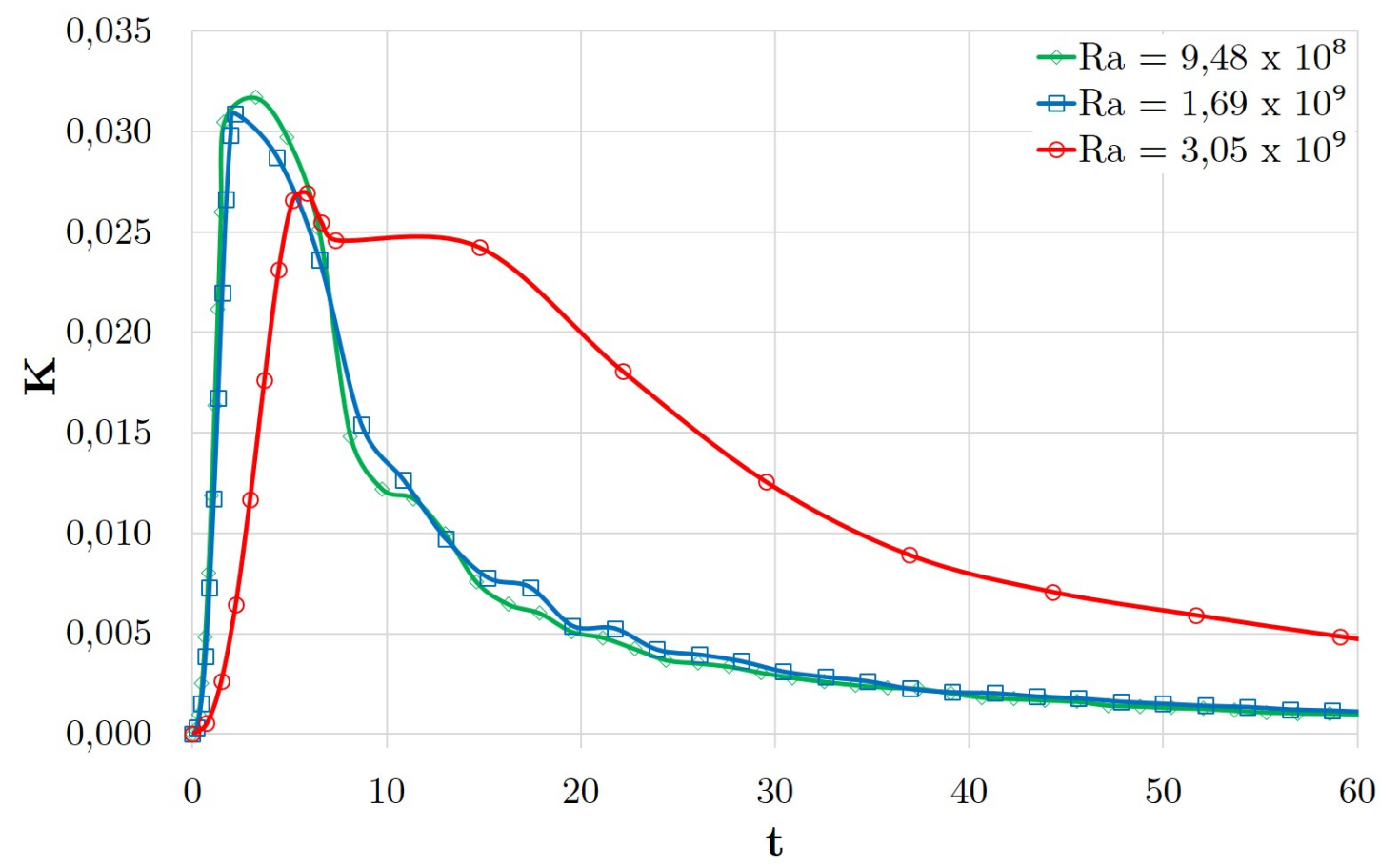

Figura 4.20: Variação da Energia Cinética no Paralelepípedo para Diferentes $T_{W}$.

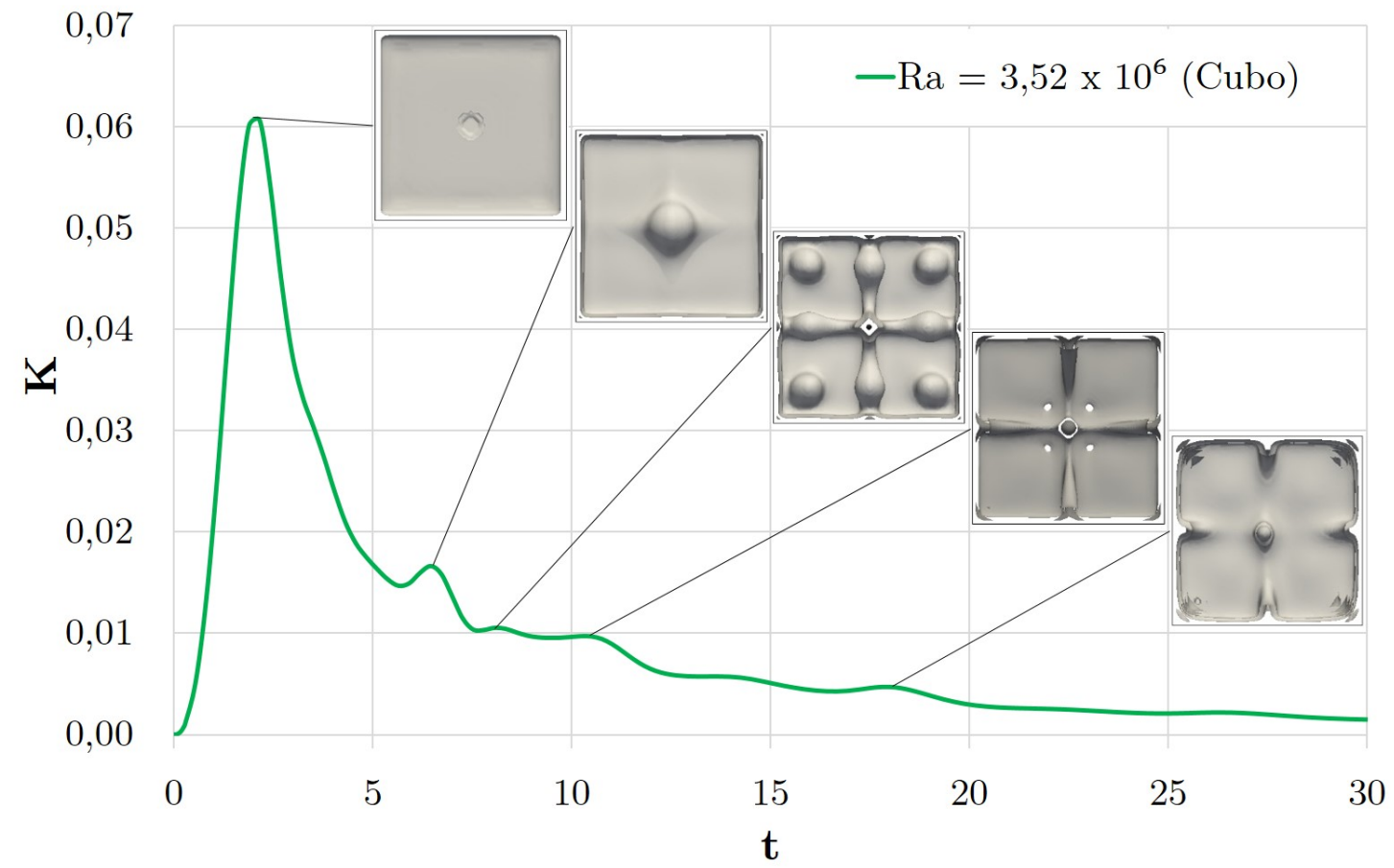

Figura 4.21: Formação de Padrões Relacionados aos Picos de Energia Cinética no Cubo. 


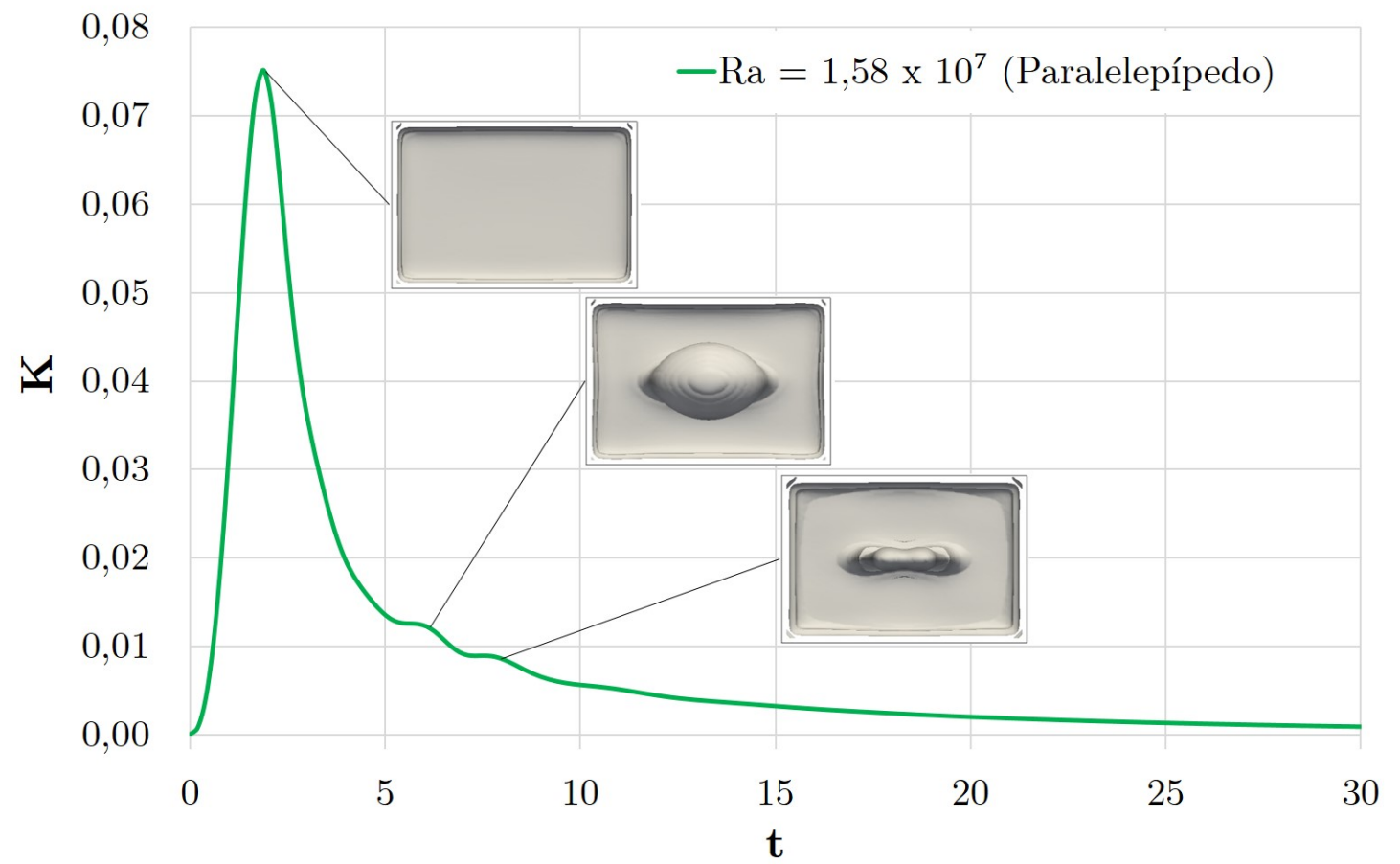

Figura 4.22: Formação de Padrões Relacionados aos Picos de Energia Cinética no Paralelepípedo.

\subsubsection{Distribuição de Nusselt nas Paredes}

Apesar das condições de contorno serem as mesmas para as seis faces do cubo, o fluxo de calor através da parede inferior e superior ocorre num processo bem distinto. Ao observarmos a Fig. (4.23), que nos mostra a distrição pontual para um determinado instante, pode-se notar que os valores dos contornos de Nusselt são muito maiores na face inferior quanto ao da face superior, onde temos um gradiente de temperatura menos elevado. 


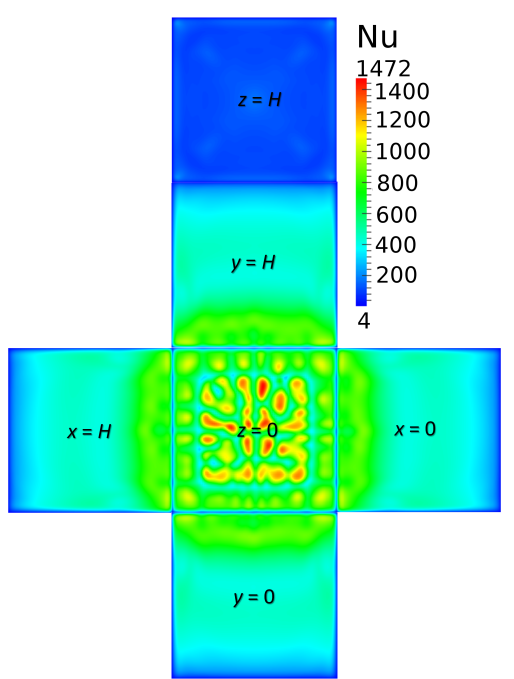

(a) $\mathrm{Ra}=7,22 \cdot 10^{7}$ em $t=4,8$

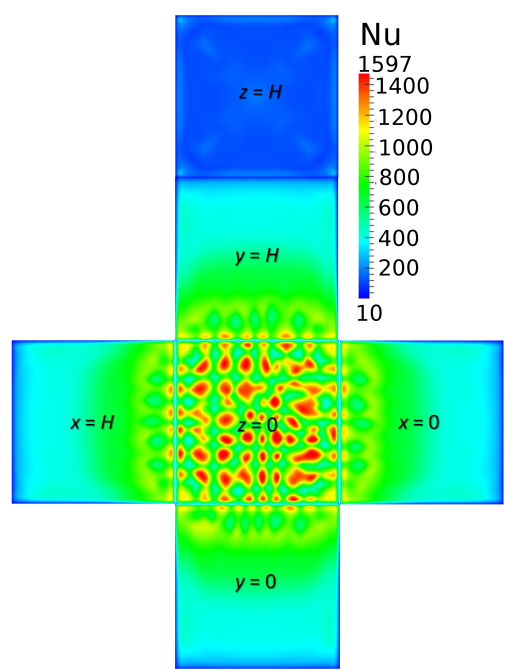

(b) $\mathrm{Ra}=1,44 \cdot 10^{8}$ em $t=5,1$

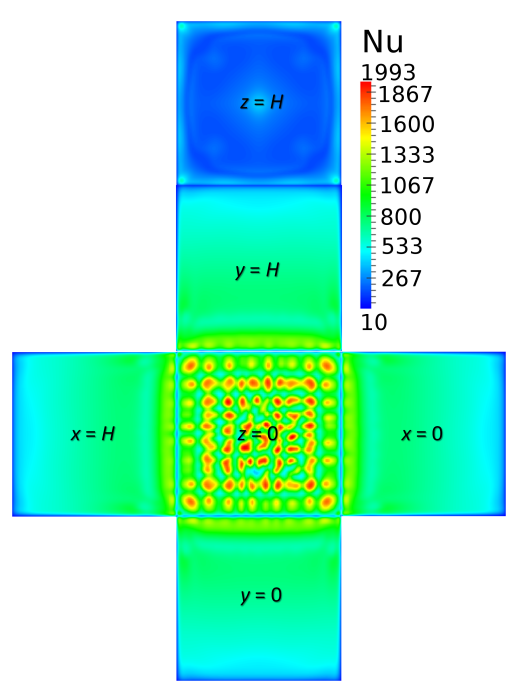

(c) $\mathrm{Ra}=2,17 \cdot 10^{8}$ em $t=4,2$

Figura 4.23: Visualização da Variação de Nusselt nas Faces do Cubo.

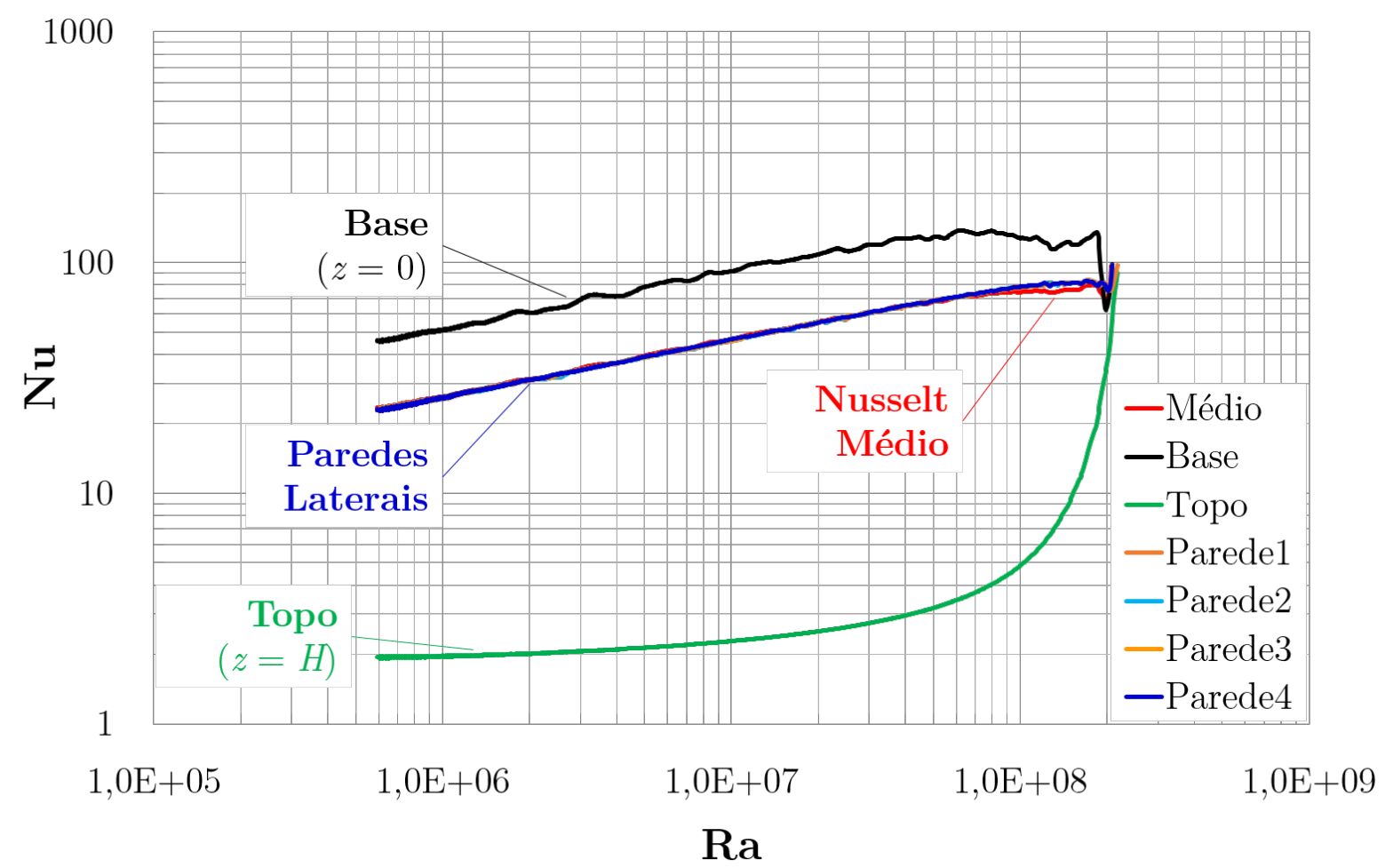

Figura 4.24: Variação de Nusselt nas Faces do Cubo.

A Fig. (4.24) apresenta os resultados médios do número Nusselt para cada face e o 
valor médio de todas as faces, variando com o número de Rayleigh. A mesma variação do valor de Nusselt entre a parede inferior e superior é observado. Além disso, as paredes laterais têm a mesma distribuição média de Nusselt.

\subsubsection{Simetria}

Como pode ser observado anteriormente, a convecção natural cria um conjunto de células de fluido que se movem circulando e formando padrões. As Figuras 4.25, 4.26, 4.27 , 4.28, 4.29, 4.30, 4.31 e 4.32 mostram alguns desses padrões, tanto para o campo de temperatura, como para o campo de pressão.

Esses resultados foram obtidos para o cubo (4 pol.) com os seguintes números de Rayleigh: $\mathrm{Ra}=2 \times 10^{5}(\Delta T=0,01), \mathrm{Ra}=2 \times 10^{6}(\Delta T=0,1), \mathrm{Ra}=4 \times 10^{6}(\Delta T=0,25)$, $\mathrm{Ra}=7,04 \times 10^{6}(\Delta T=0,5), \mathrm{Ra}=2 \times 10^{7}(\Delta T=1), \mathrm{Ra}=8 \times 10^{7}(\Delta T=5), \mathrm{Ra}=7 \times 10^{8}$ $(\Delta T=45)$; e para o paralelepípedo: $\mathrm{Ra}=1,58 \times 10^{7}(\Delta T=0,25)$. Esses parâmetros foram definidos após várias sequências de simulações e observações, de maneira a visualizar o comportamento dos padrões que se formam na cavidade, à medida em que aumentávamos gradativamente o número de Rayleigh (ou aumentávamos a diferença de temperatura). Para evidenciar essas estruturas, optamos por utilizar os contornos de temperatura $(T)$ e o sombreamento de pressão $(p)$ que conseguiram demarcar com mais evidências as seções do cubo ou paralelepípedo retangular.
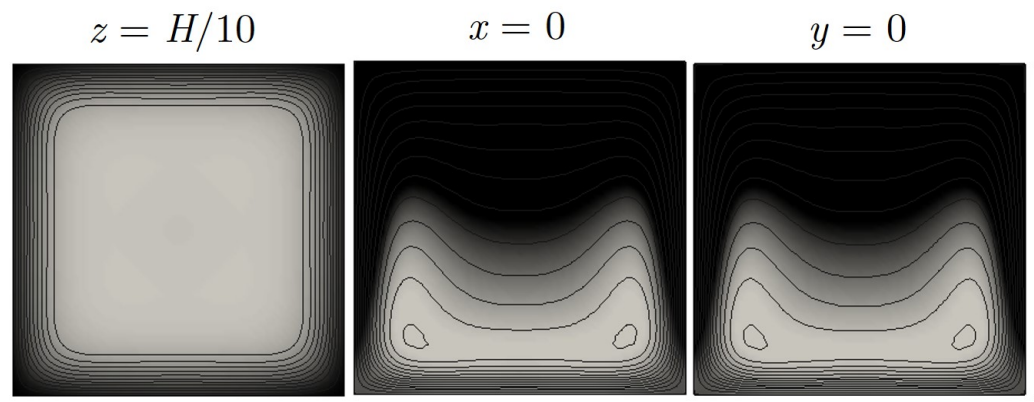

Vista superior

$$
t=560 \mathrm{~s}
$$

Contornos de $T$ e sombreamentos de $p$

Isosuperfícies de $p$

Figura 4.25: Visualização da simetria no cubo para $\mathrm{Ra}=2 \times 10^{5}$ 


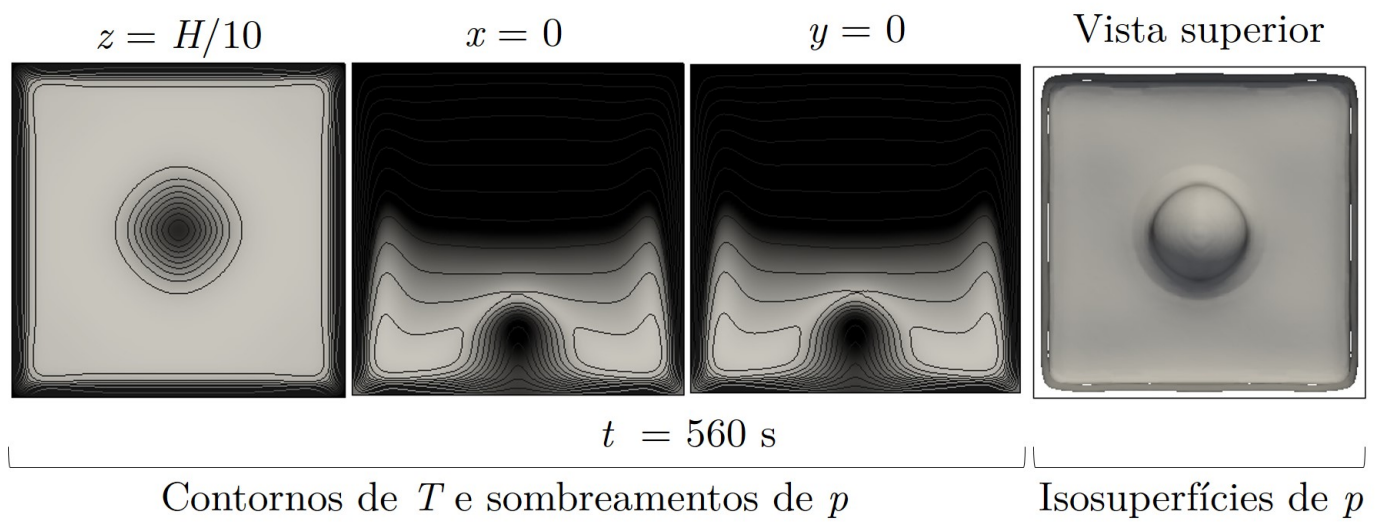

Figura 4.26: Visualização da simetria no cubo para $\mathrm{Ra}=2 \times 10^{6}$

Os padrões aparecem, principalmente, quando observamos um plano perpendicular ao eixo $z$ em $H / 30 \leqslant z \leqslant H / 10$ e se formam logo no início do processo de convecção natural e do aparecimento das bolhas de fluido em forma de cogumelo. Logo após o fluido começar a perder a energia cinética inicial (Fig. (4.19)), as cavidades com número de Raylaigh elevado, ocorre a perda de simetria.

A primeira figura (Fig. 4.25 nos apresenta resultados para $\mathrm{Ra}=2 \times 10^{5}$ em que a variação de temperatura é de $\Delta T=0,01$. Mesmo com essa variação tão pequena, o fluido no interior da cavidade se aquece, ocasionando a estratificação da temperatura, mostradas nas seções em $x=0$ e $y=0$, mas, os gradientes de temperatura não são suficientes para a formação das bolhas de fluido aquecido. Na sequência, a Fig. 4.26, com Ra $=2 \times 10^{6}(\Delta T=0,1)$, mostra o surgimento de uma bolha central que se forma logo após $1 / 3$ do tempo de simulação e permanece até o final do processo de convecção.

O número de $\mathrm{Ra}=4 \times 10^{6}(\Delta T=0,25)$ é o que apresenta a transição de uma única bolha para a disposição das bolhas na forma de uma matriz $3 \times 3$. Essa transição foi mostrada na Fig. (4.21) e, apesar da variação do perfil dos contornos de temperatura a simetria permanece até o término da simulação. Utilizando o mesmo número de Rayleigh para o paralelepípedo retangular, observa-se a predominância da única bolha de fluido aquecido, mantendo-se até o fim da convecção na cavidade.

A partir do número de $\mathrm{Ra}=7,04 \times 10^{6}(\Delta T=0,5)$ e $\mathrm{Ra}=2 \times 10^{7}(\Delta T=1)$, temos a predominância do surgimento das estruturas na forma de uma matriz $3 \times 3$, mas, com a indicação da perda de simetria no final do processo de convecção natural. Para $\mathrm{Ra}=8 \times 10^{7}(\Delta T=5)$ e $\mathrm{Ra}=7 \times 10^{8}(\Delta T=45)$ em diante, percebe-se a perda de 


\section{Resultados}

simetria já no início do aquecimento da cavidade.

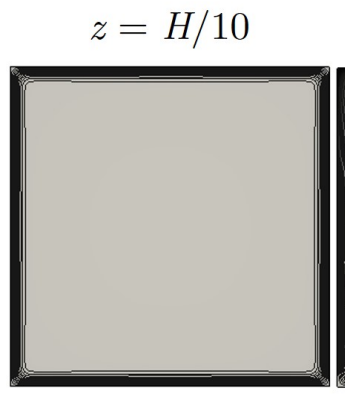

$x=H / 2$

$y=H / 2$

Vista superior

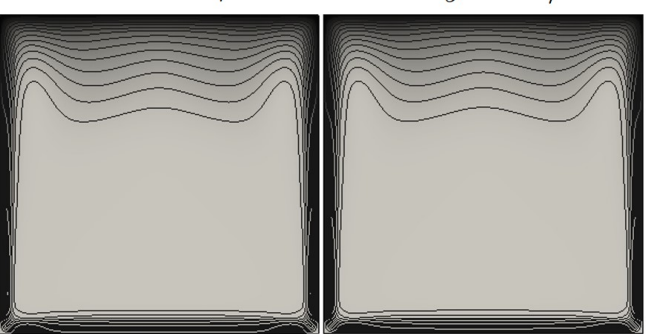

(a) $t=80 \mathrm{~s}$
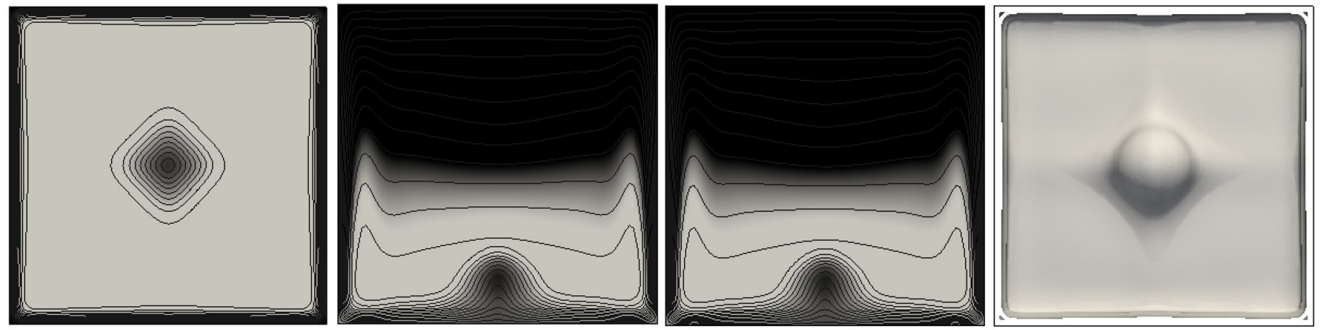

(b) $t=210 \mathrm{~s}$
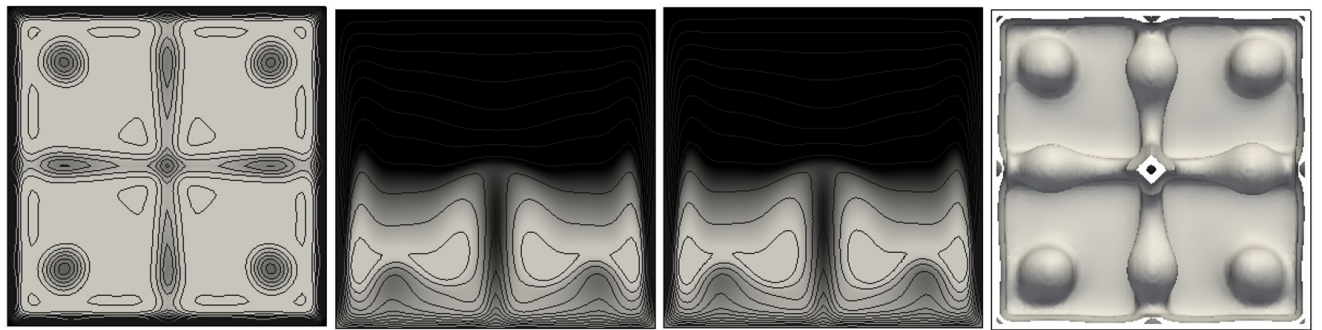

(c) $t=310 \mathrm{~s}$
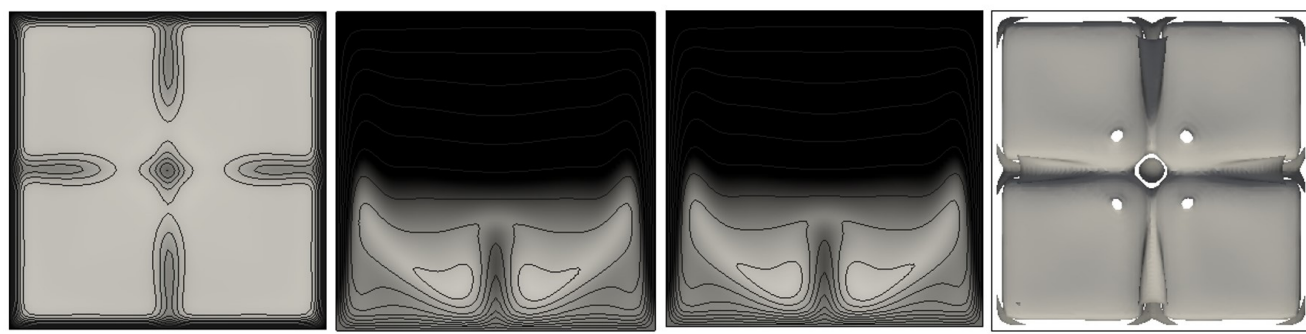

(d) $t=430 \mathrm{~s}$
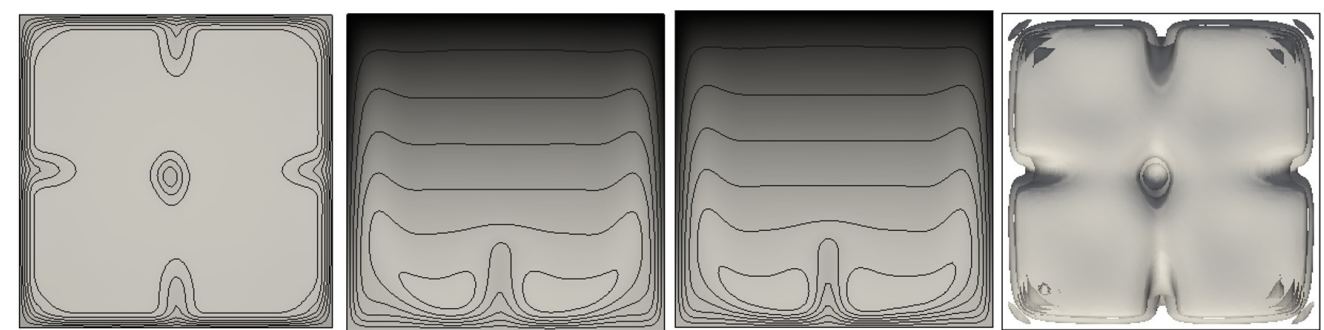

(e) $t=670 \mathrm{~s}$

Contornos de $T$ e sombreamentos de $p$ Isosuperfícies de $p$

Figura 4.27: Visualização da simetria no cubo para $\mathrm{Ra}=4 \times 10^{6}$ 


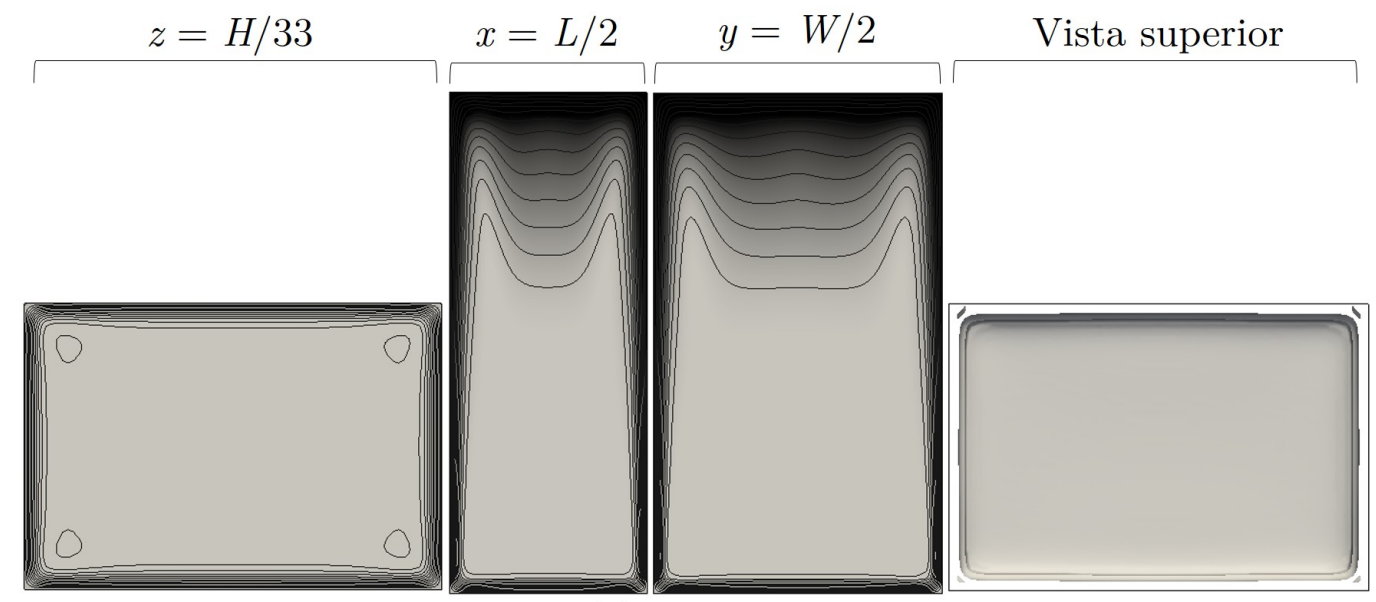

(a) $t=90 \mathrm{~s}$

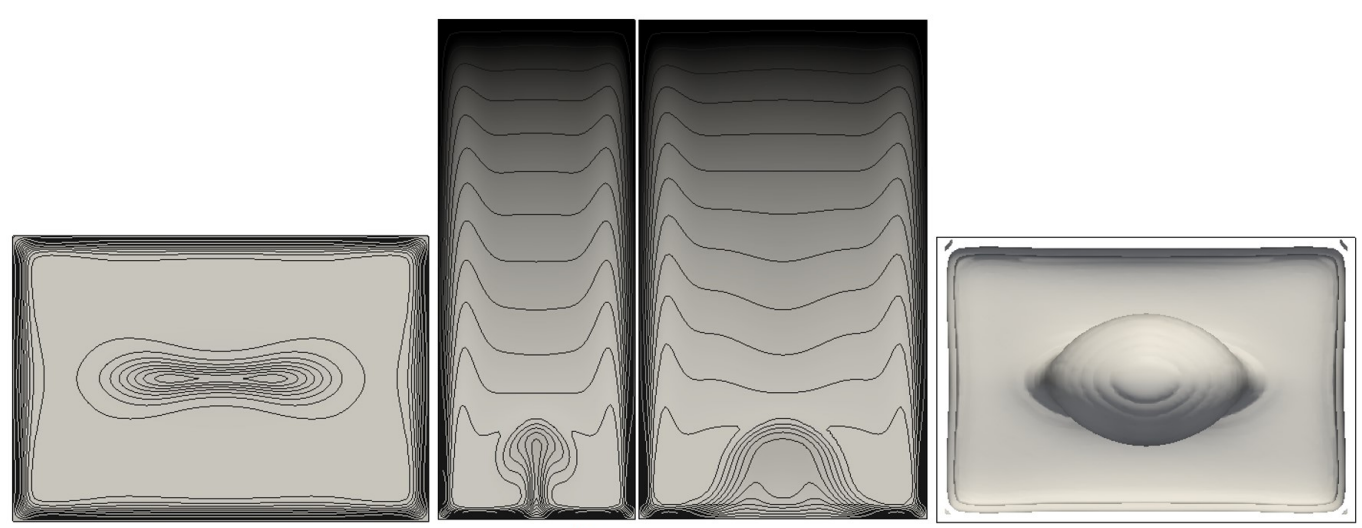

(b) $t=270 \mathrm{~s}$

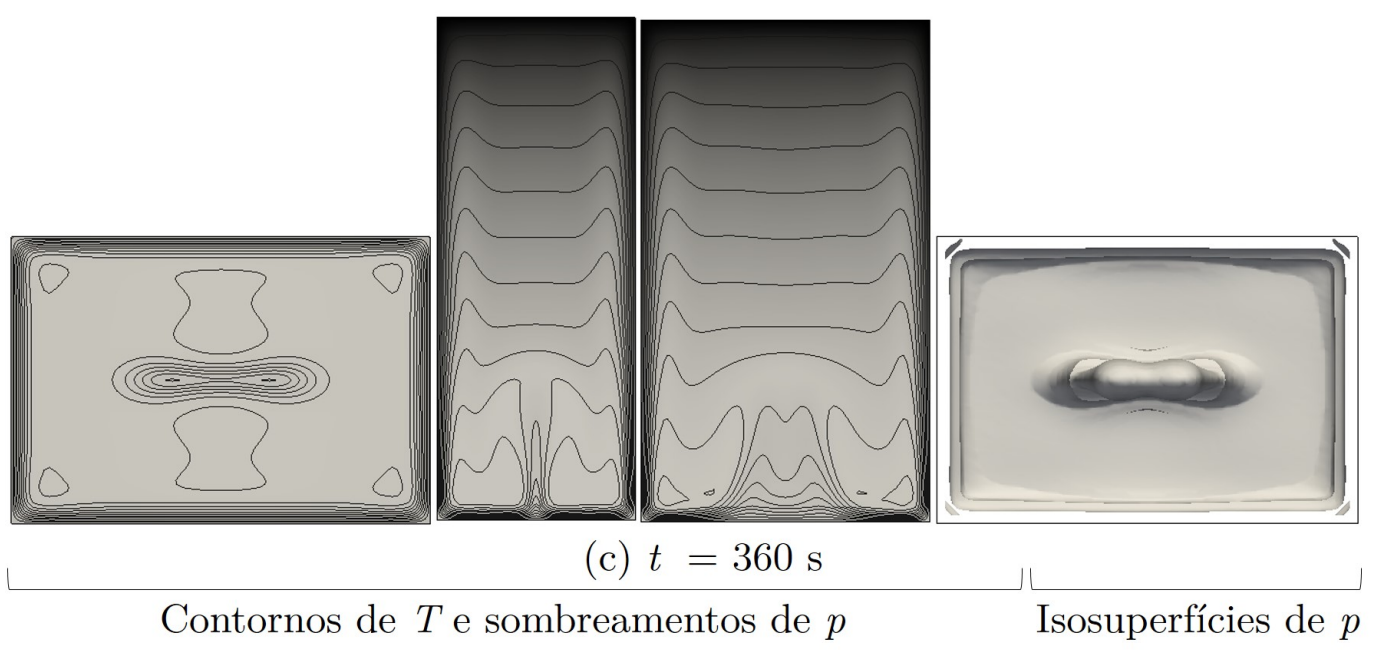

Figura 4.28: Visualização da simetria no Paralelepípedo para $\mathrm{Ra}=1,58 \times 10^{7}$ 
$z=H / 10$
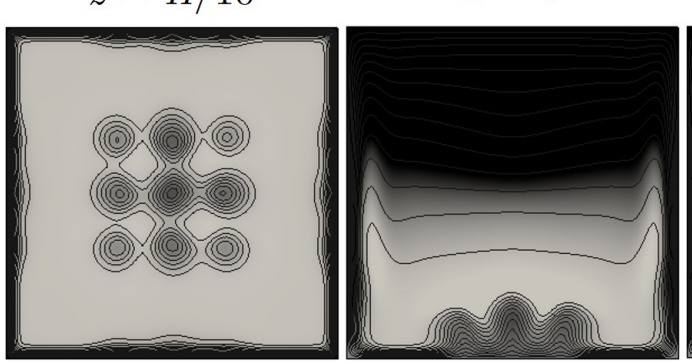

(a) $t=150 \mathrm{~s}$

$y=0$
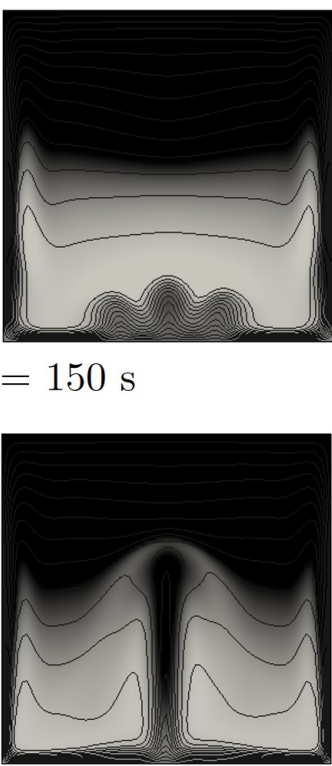

(b) $t=210 \mathrm{~s}$
Vista superior
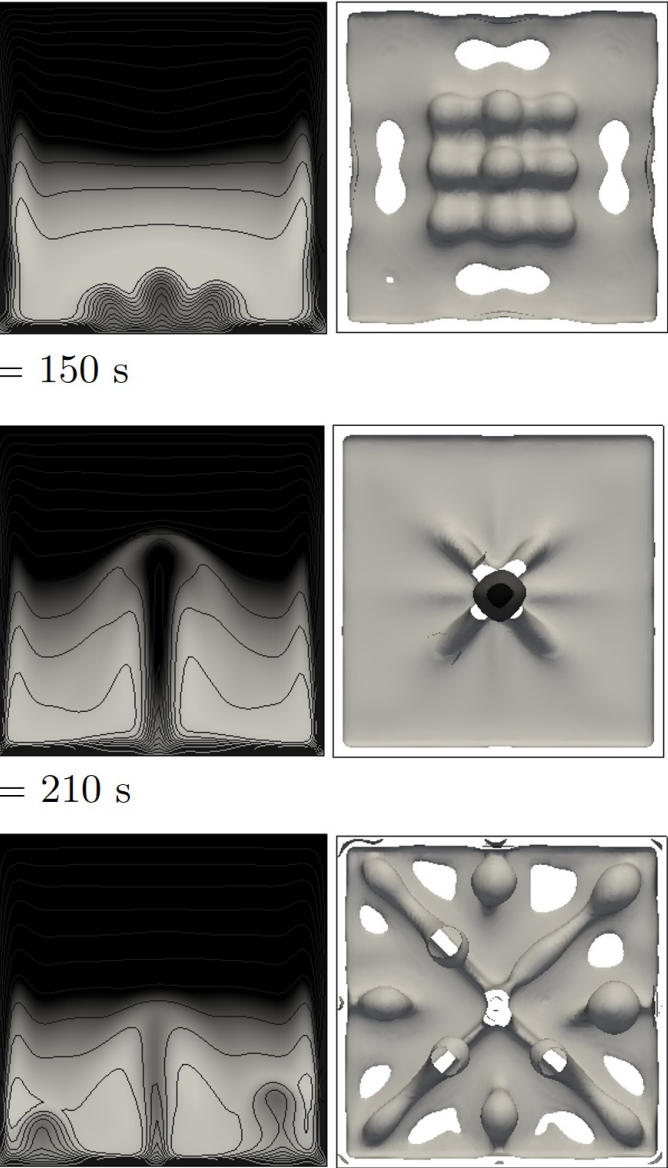

(c) $t=300 \mathrm{~s}$

Contornos de $T$ e sombreamentos de $p$

Figura 4.29: Visualização da simetria no cubo para $\mathrm{Ra}=7,04 \times 10^{6}$ 


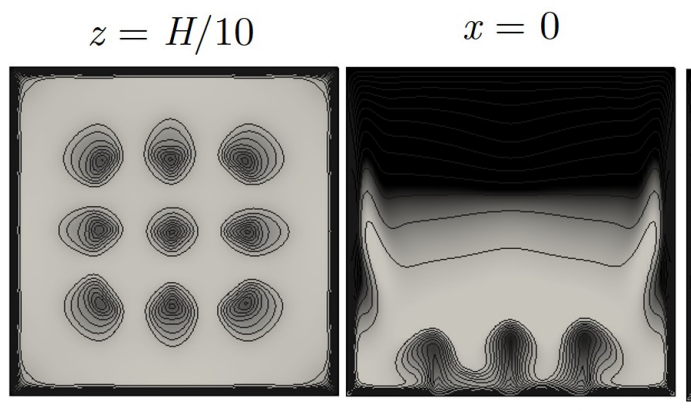

$y=0$

Vista superior

(a) $t=100 \mathrm{~s}$
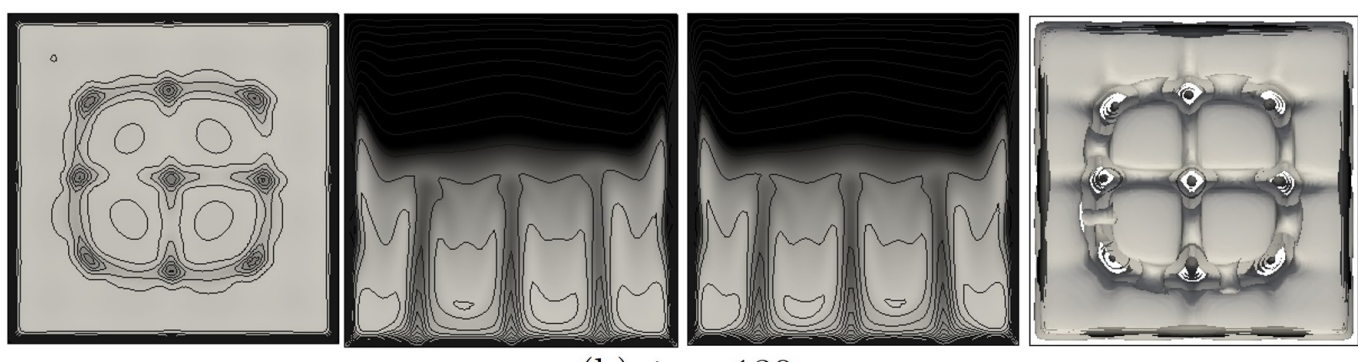

(b) $t=130 \mathrm{~s}$
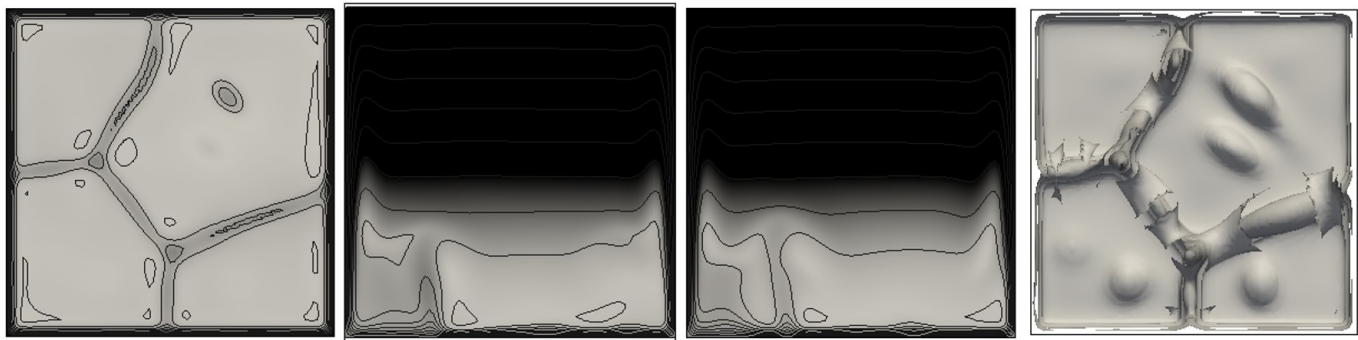

(c) $t=310 \mathrm{~s}$

Contornos de $T$ e sombreamentos de $p$ Isosuperfícies de $p$

Figura 4.30: Visualização da simetria no cubo para $\mathrm{Ra}=2 \times 10^{7}$ 
$z=H / 10$

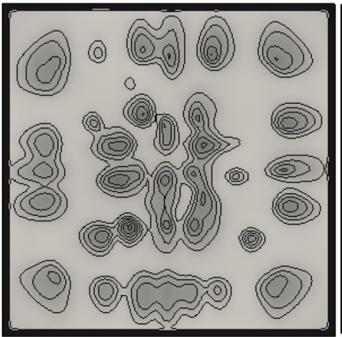

9) $\sqrt{(2 \pi 0)}(0)$ $x=0$

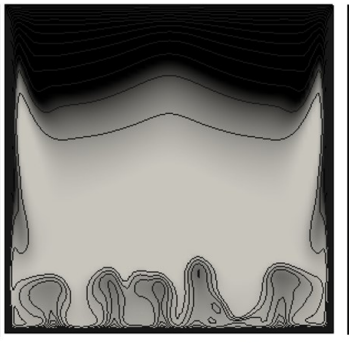

(a) $t=40 \mathrm{~s}$ $y=0$
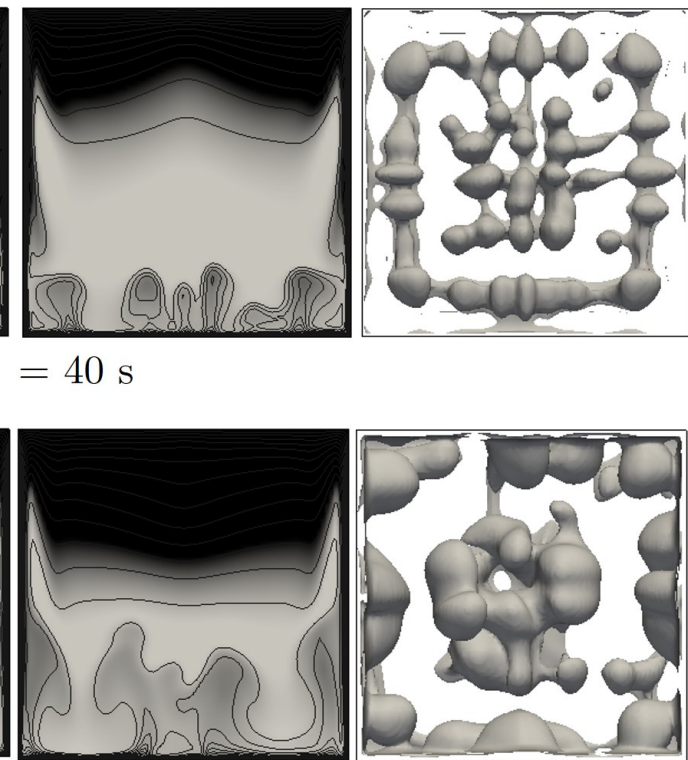

(b) $t=50 \mathrm{~s}$
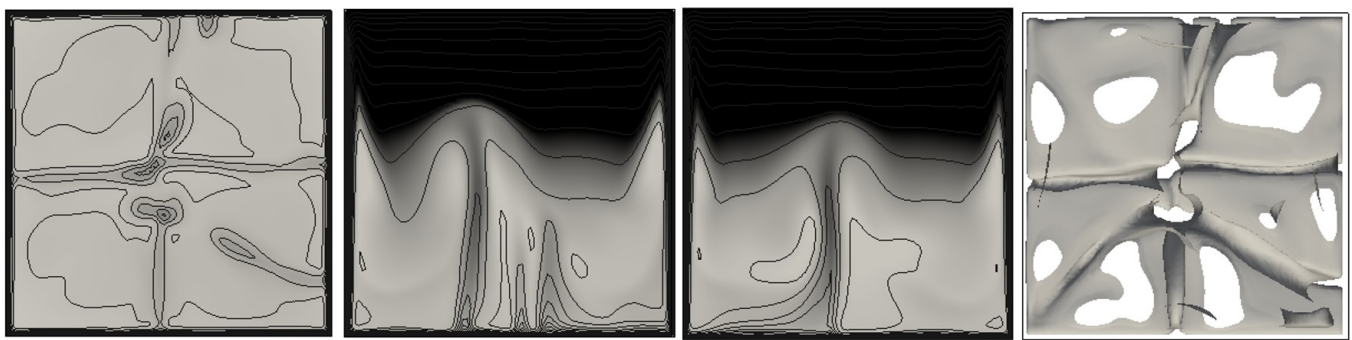

(c) $t=70 \mathrm{~s}$

Contornos de $T$ e sombreamentos de $p$ Isosuperfícies de $p$

Figura 4.31: Visualização da simetria no cubo para $\mathrm{Ra}=8 \times 10^{7}$ 

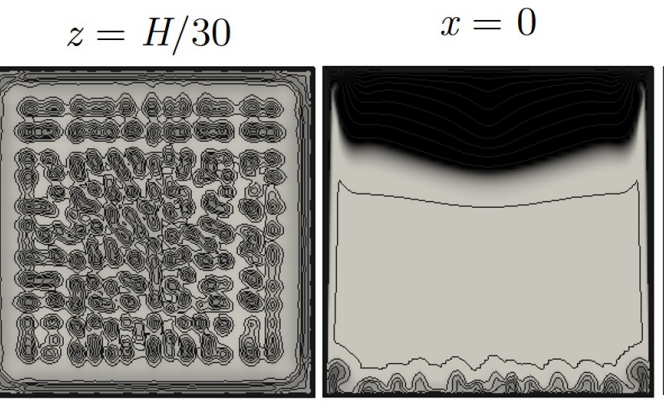

(a) $t=10 \mathrm{~s}$
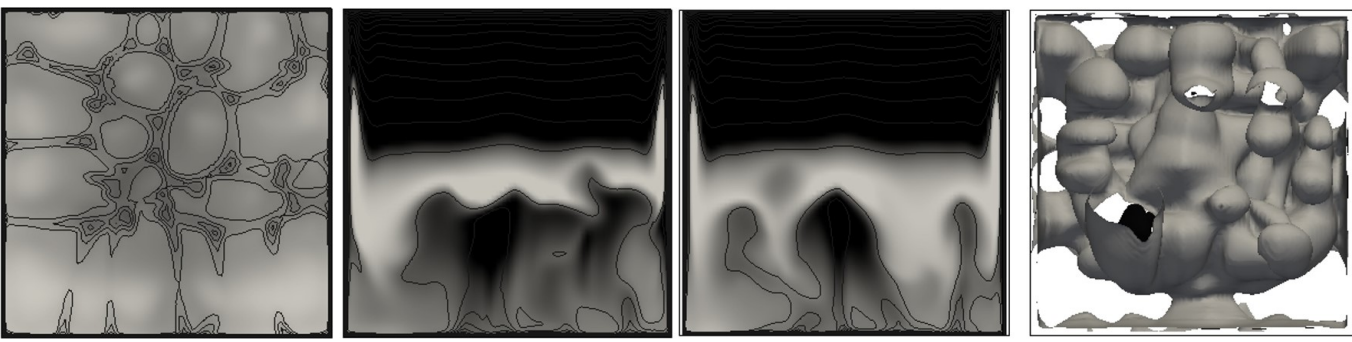

(b) $t=20 \mathrm{~s}$
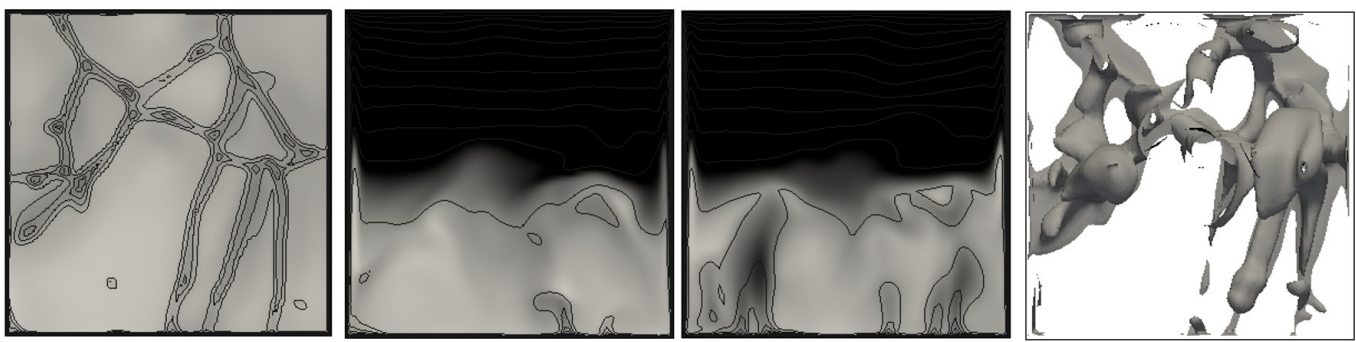

(c) $t=40 \mathrm{~s}$

Contornos de $T$ e sombreamentos de $p$ Isosuperfícies de $p$

Figura 4.32: Visualização da simetria no cubo para $\mathrm{Ra}=7 \times 10^{8}$

\subsection{Efeitos da Agitação no Processo de Convecção Natural}

Nesta seção analisaremos o processo de agitação imposto à cavidade ao variarmos seu ângulo de inclinação com o tempo $(\phi(t))$, estabelecido na Eq. 2.67). A fim de verificar a interferência entre a agitação ou rotação com o processo de convecção natural, várias configurações definidas entre $\Omega(t)$ (Eq. 2.69) e $\phi(t)$ foram testadas (Tabela 4.4). 


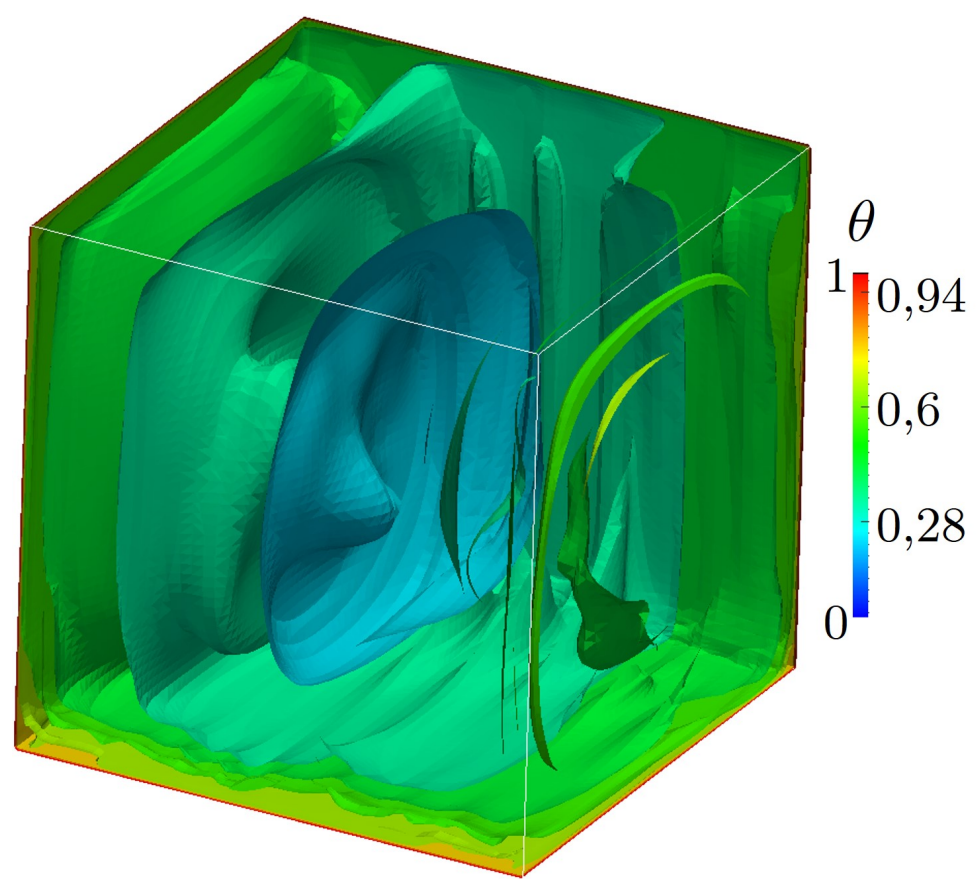

Figura 4.33: Agitação do Fluido devido a $\phi(t)$ - Isosuperfícies de $\theta$

O que se espera com o processo de agitação/rotação da cavidade é que as estruturas aquecidas que se formam na cavidade se quebrem e se misturem mais rapidamente com parte do fluido próximo à parede. Assim, a transferência de calor ocorra com maior rapidez 4.33 .

O parâmetro que caracteriza a aceleração no aquecimento da cavidade foi estabelecido através do tempo adimensional, dado pela equação

$$
t^{*}=\frac{t}{H} \sqrt{\frac{g \beta\left(T_{w}-T_{0}\right) H \alpha}{\nu}}
$$

E um tempo mínimo, $t_{\text {min }}$, associado ao tempo necessário para atingir $80 \%$ da temperatura $T_{W}$.

Na Figura (4.34) temos as curvas de temperatura $(\theta)$ variando com o tempo $(t)$, ambos na forma adimensional. Neste gráfico foram mostrados os valores da temperatura de acordo com $\phi$ fixo (ou seja, sem agitação, apenas a cavidade foi inclinada), estabelecido em $0^{\circ}, 30^{\circ}$ e $60^{\circ}$; outros resultados são apresentados impondo a agitação à cavidade, fazendo $\Omega=1 / 2,1,5 / 4,3 / 2,2$ e 3 para $\phi_{\max }=30^{\circ}$ e $60^{\circ}$. 
Tabela 4.4: Parâmetros Utilizados na Simulação da Agitação da Cavidade

\begin{tabular}{|c|c|c|c|c|}
\hline \multicolumn{2}{|c|}{ Variáveis } & \multicolumn{3}{|c|}{$\phi(t)$} \\
\hline$T_{0}[K]$ & 294,16 & Tipo & $\phi[\mathrm{rad}]$ & $\Omega[\mathrm{rad} / \mathrm{s}]$ \\
\hline$T_{W}[K]$ & 297,16 & \multirow{6}{*}{ Oscilação } & \multirow{6}{*}{$\pi / 6$ e $\pi / 3$} & 0,5 \\
\hline$h[m]$ & 0,1016 & & & 1 \\
\hline$g_{0}\left[\mathrm{~m} / \mathrm{s}^{2}\right]$ & 9,81 & & & 1,25 \\
\hline$\beta\left[K^{-1}\right]$ & $2,16 \cdot 10^{-4}$ & & & 1,5 \\
\hline $\operatorname{Pr}$ & 6,81 & & & 2 \\
\hline$\alpha\left[m^{2} / s\right]$ & $1,44 \cdot 10^{-7}$ & & & 3 \\
\hline$\nu\left[m^{2} / s\right]$ & $9,80 \cdot 10^{-7}$ & Fixo & $0, \pi / 6$ e $\pi / 3$ & 0 \\
\hline$\rho\left[\mathrm{kg} / \mathrm{m}^{3}\right]$ & 998,0 & & & \\
\hline $\mathrm{Ra}^{*}$ & $4,74 \cdot 10^{-7}$ & & & \\
\hline$U_{0}[\mathrm{~m} / \mathrm{s}]$ & $9,75 \cdot 10^{-3}$ & & & \\
\hline$K_{0}\left[\mathrm{~m}^{2} / \mathrm{s}^{2}\right]$ & $4,74 \cdot 10^{-2}$ & & & \\
\hline
\end{tabular}




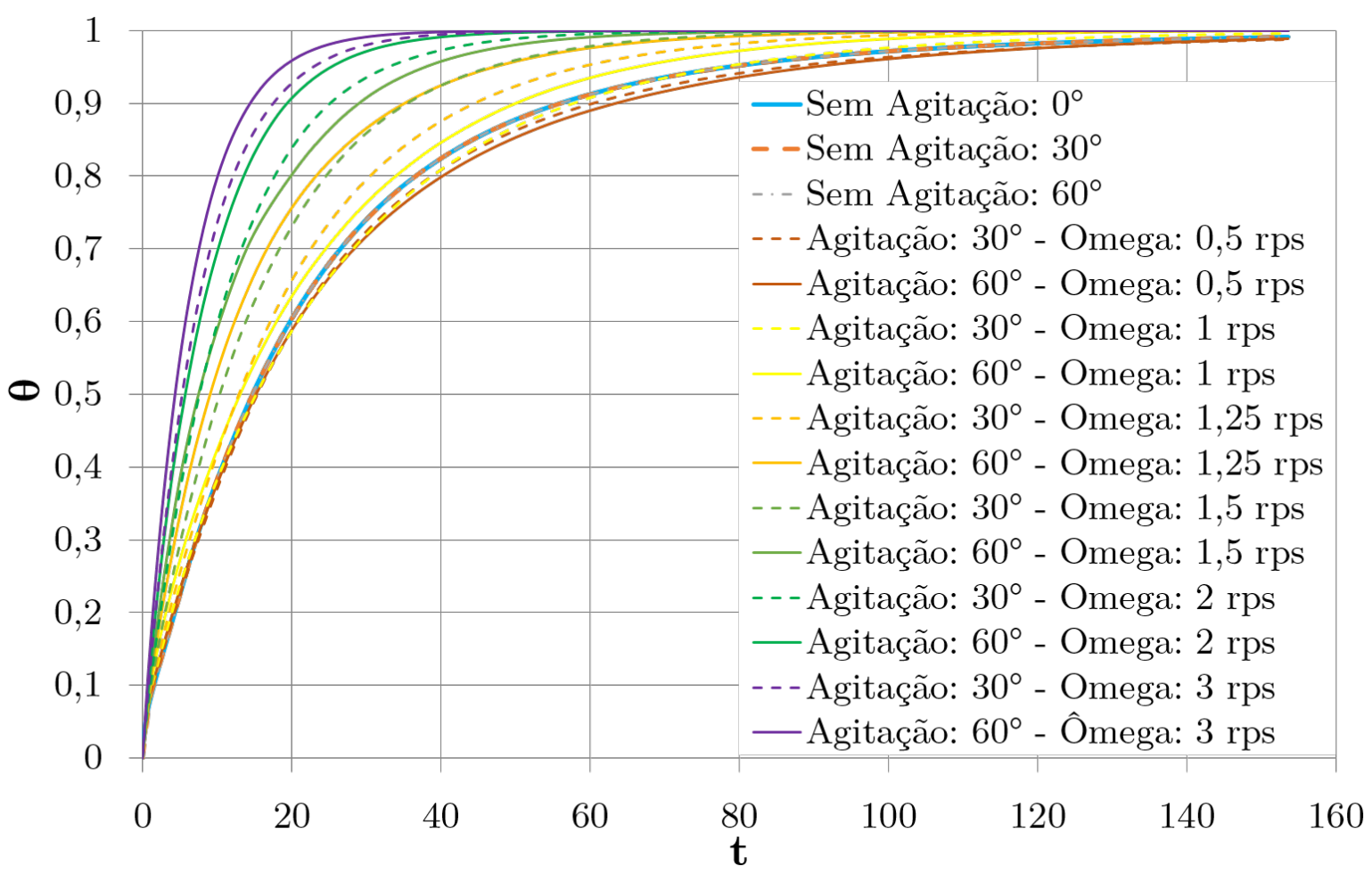

Figura 4.34: Influência da Oscilação na Convecção Natural - Temperatura.

O que pode ser observado é que os efeitos da agitação no processo de convecção são percebidos para valores de $\Omega$ acima de 1,25, combinados com uma amplitude de $\phi_{\max }$ em $60^{\circ}$. Isso se deve à quebra das estruturas e a uma mistura mais rapidamente das camadas de fluido frias com as já aquecidas, acelerando o processo de convecção natural (Fig. 4.33 e mantendo o número de Nusselt constante ao longo da simulação (Fig. 4.35). 


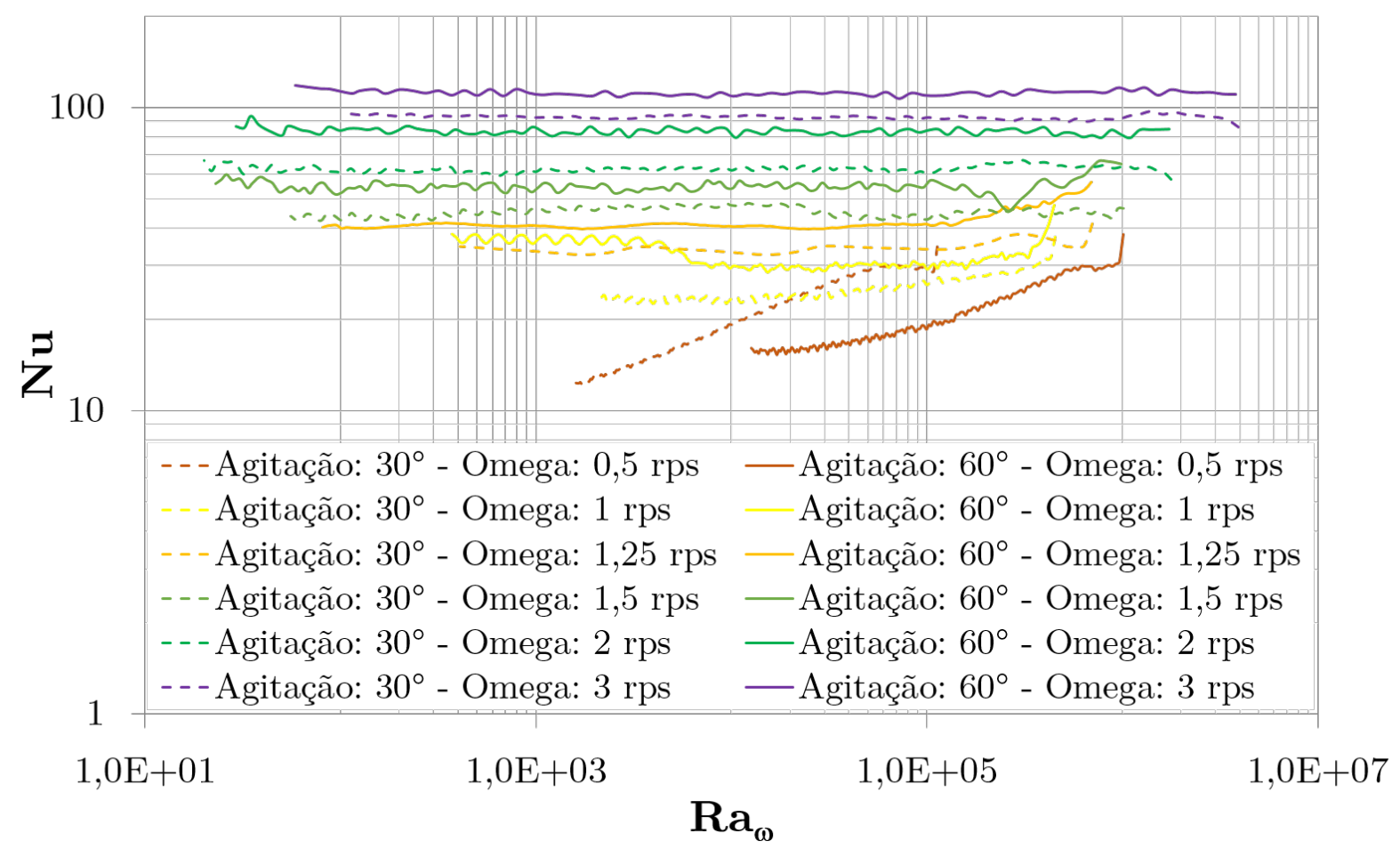

Figura 4.35: Influência da Oscilação na Convecção Natural - Nusselt.

A figura 4.36 ilustra a influência da inclinação e da agitação com o decaimento do tempo para atingir $80 \%$ da temperatura de aquecimento das paredes, $T_{W}$.

Conforme se esperava, uma maior agitação da cavidade, vinculado com a inclinação máxima, fez com que o tempo de aquecimento caísse.

Apesar dos resultados representarem um fator positivo em relação à agitação, será necessário uma análise mais detalhada da capacidade do equipamento que gerará a agitação e o tipo de alimento a ser agitado. 


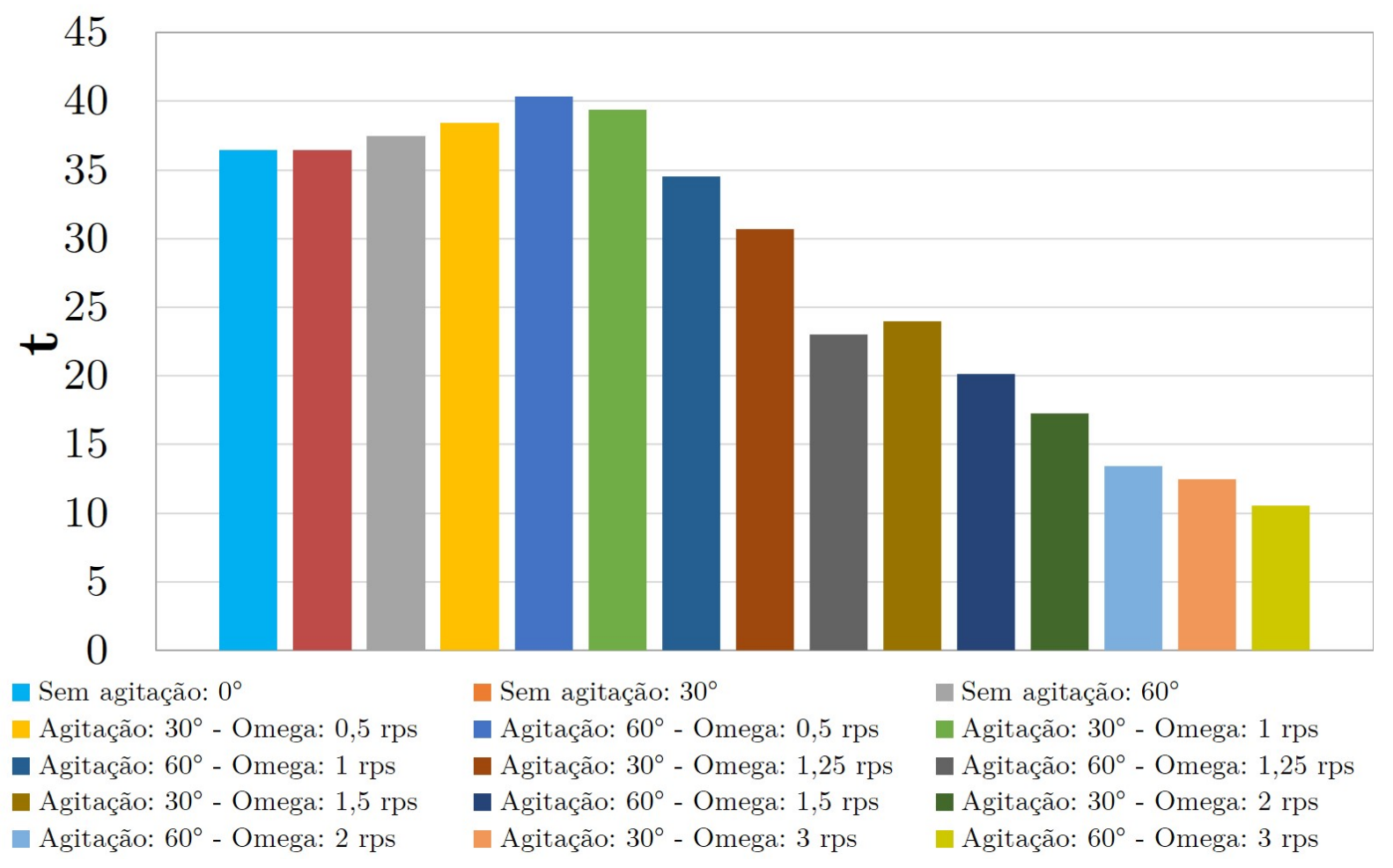

Figura 4.36: Influência da Oscilação na Convecção Natural - Temperatura.

\subsection{Turbulência}

A simulação da convecção natural na cavidade possui uma gama de resultados e correlações, dependendo da condição em que as paredes são aquecidas ou resfriadas e a relação com seu raio de aspecto.

Na situação em que todas as faces são aquecidas, verificamos que a análise das regiões I e II, mostradas na figura (1.3) seguem, respectivamente, as interpretações obtidas dos casos em que temos uma placa vertical e uma placa plana aquecida.

A cavidade paralelepípeda retangular de (TOLLINI, 1996) possui a característica de ter $H>L$, consequentemente, pressupõe-se que a influência causada pelas paredes verticais seja maior no processo de transferência de calor.

Nos resultados simulados e na suspeita apresentada por (TOLLINI, 1996) o número de 
Rayleigh atinge patamares que estão associados a convecção natural que transiciona de laminar para turbulento. Para esta situação não há na literatura correlações de $\mathrm{Nu}$ vs Ra indicadas para a turbulência.

Conforme observamos, as paredes verticais da cavidade têm um papel importante na convecção natural. Então, supomos a correlação de $\mathrm{Nu}$ vs Ra utilizada na como padrão na literatura, $\mathrm{Nu}=0,1 \mathrm{Ra}^{1 / 3}$ para valores de $\mathrm{Ra}>10^{9}$ 4.37. Os modelos de turbulência escolhidos foram o modelo $k-\epsilon$ e modelo Sub-Malha de Smagorinsky.

O Solver padrão de convecção natural do OpenFOAM não vem com suporte para os modelos de Simulação de Grandes Escalas. Por este motivo, implementou-se um novo Solver, o LES-buoyantBoussinesPimpleFoam com base no original, em que foram adicionadas as bibliotecas já existentes do modelo de LES no código.

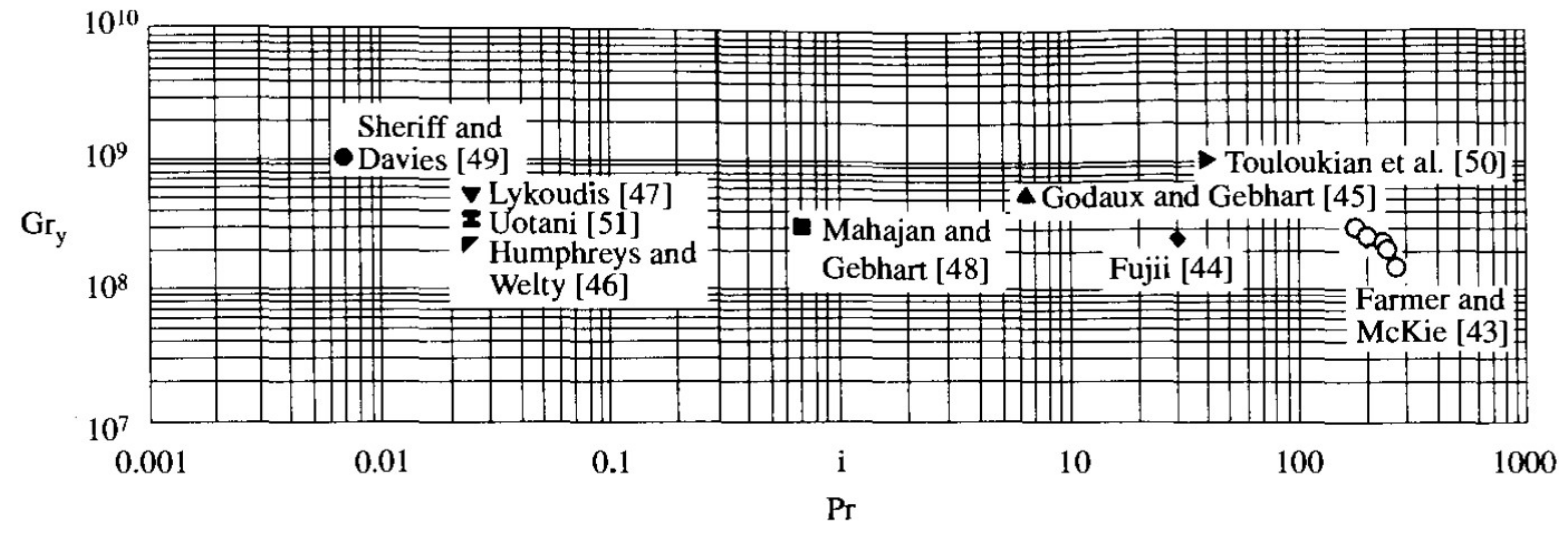

Figura 4.37: Critério para Transição à Turbulência para Placa Plana Vertical (Adptado de (BEJAN, 2004)).

Para verificar se a correlação tem aplicabilidade na cavidade de (TOLLINI, 1996), elevou-se $\Delta \mathrm{T}$ para $100{ }^{\circ} \mathrm{C}$, resultando em $\mathrm{Ra}=6,77 \times 10^{9}$. O modelo de Sub-Malha de Smagorinsky foi o que obteve melhores resultados. 


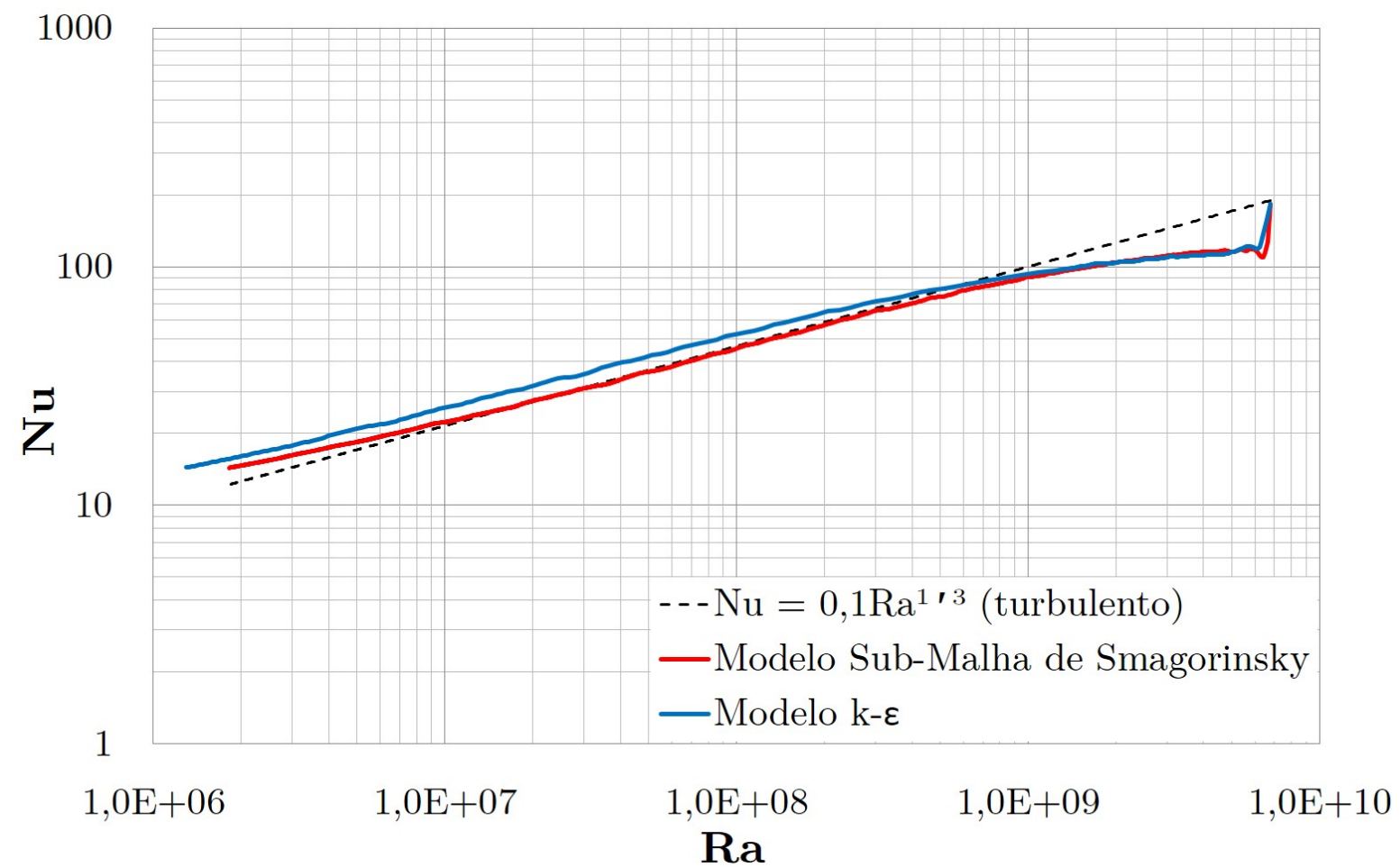

Figura 4.38: Comparação de Nusselt $\times$ Rayleigh do Cubo com dados experimentais ((LIN; AKINS, 1983)).

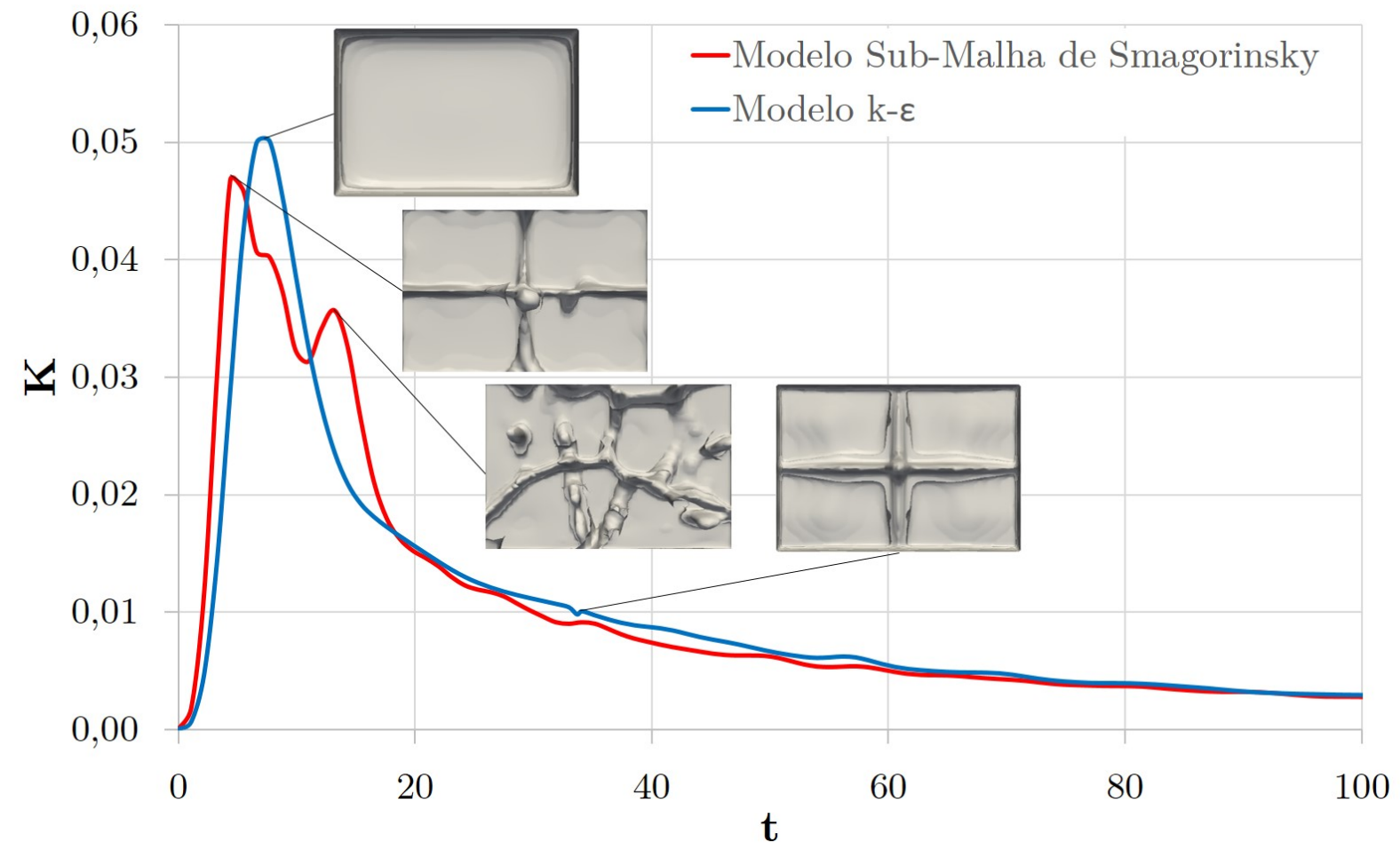

Figura 4.39: Formação de Padrões Relacionados aos Picos de Energia Cinética no Paralelepípedo para o Caso Turbulento. 
$x=L / 2$
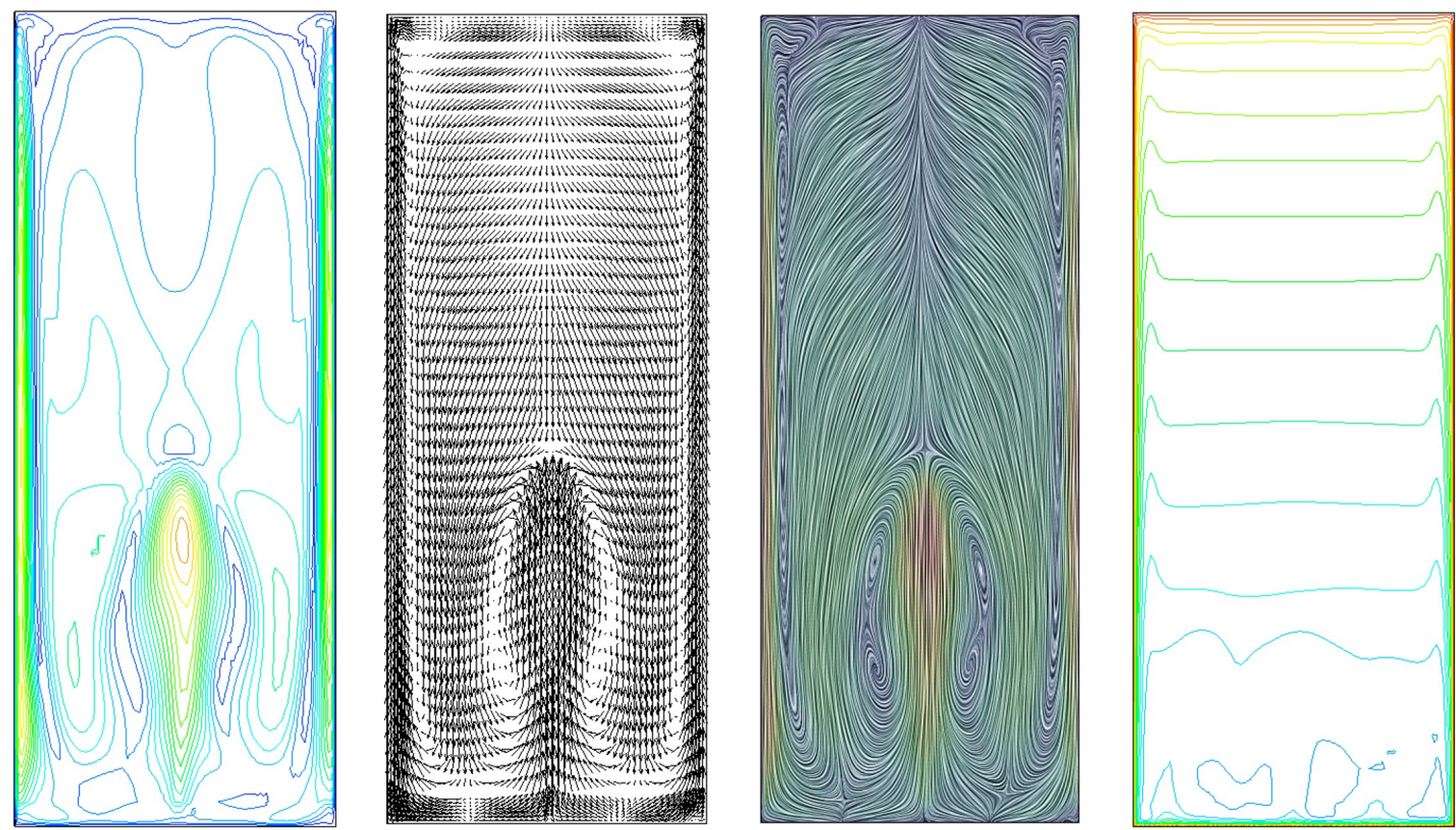

(a) $t=10 \mathrm{~s}$
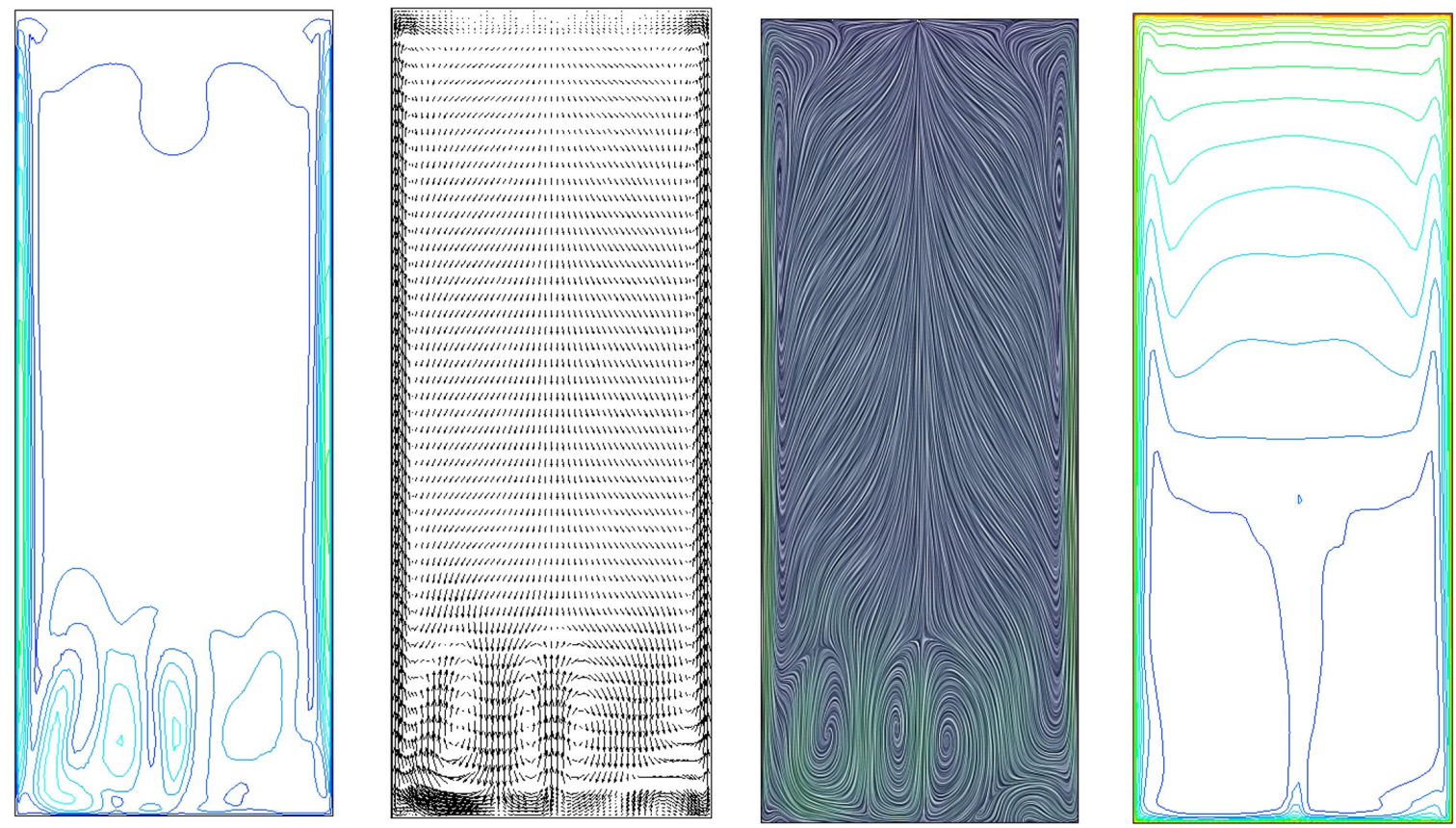

(b) $t=90 \mathrm{~s}$

Contornos de $\mathbf{u} \quad$ Vetores de $\mathbf{u}$ Linhas de corrente Contornos de $T$

Figura 4.40: Visualização das Características do Paralelepípedo Retangular em $x=$ $H / 2$ para $\mathrm{Ra}=6,77 \times 10^{9}$ 
$z=H / 33$
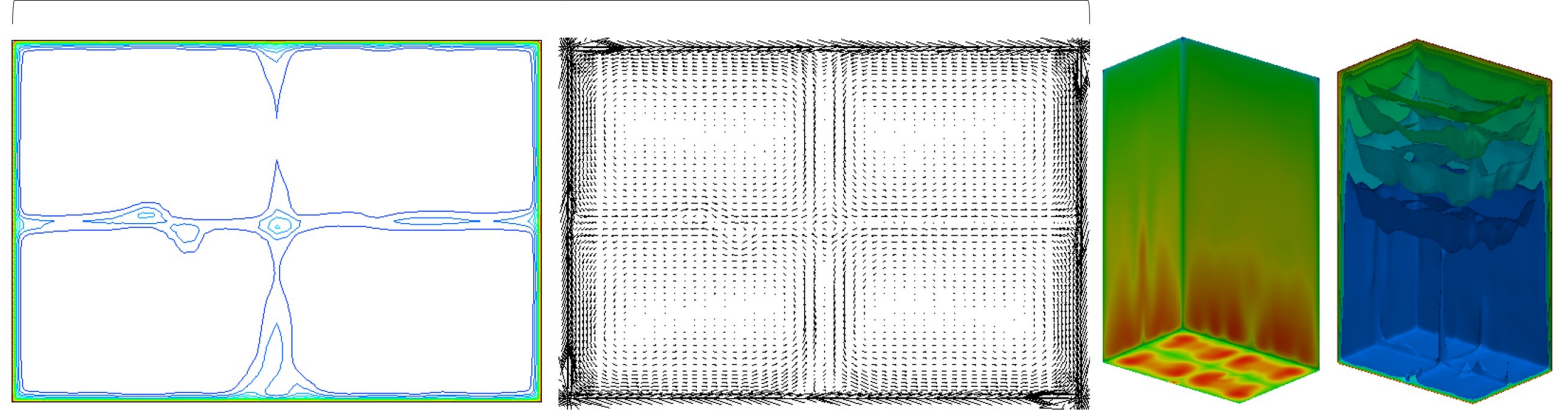

(a) $t=10 \mathrm{~s}$

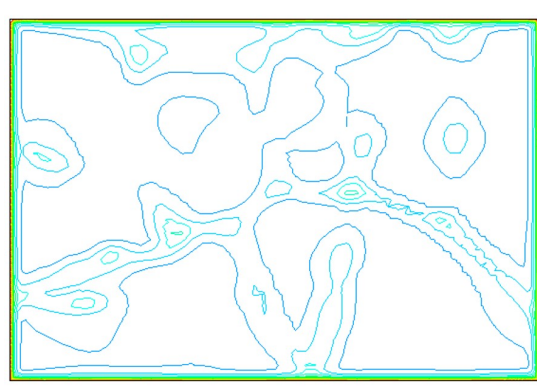

Contornos de $T$

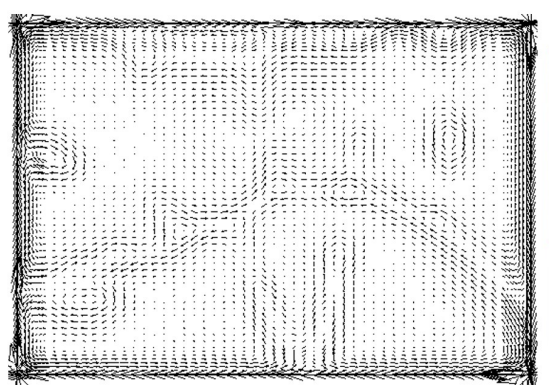

(b) $t=90 \mathrm{~s}$
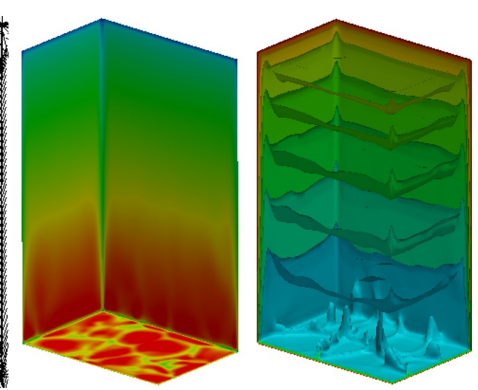

Nusselt Isosup. de $T$

Figura 4.41: Visualização das Características do Paralelepípedo Retangular em $x=$ $H / 2$ para $\mathrm{Ra}=6,77 \times 10^{9}$ 


\section{Conclusões}

\section{Capítulo 5}

\section{Conclusões}

Resultados da modelagem e simulação da convecção natural em uma cavidade paralelepípeda retangular preenchida com água, através da implementação no código do OpenFOAM, foram mostrados. O estudo de caso de dois problemas experimentais foram utilizados como base para validação do código do OpenFOAM: cavidade cúbica do trabalho de (LIN, 1982) e (LIN; AKINS, 1983) e a cavidade paralelepípeda retangular do trabalho de (TOLLINI, 1996). Foram inicialmente testadas as configurações de malha (256.000 elementos, 729.000 elementos e 1.728 .000 elementos), através de valores de $\theta \times t, \mathrm{Nu} \times t, \theta \times x$ e $U_{z} \times x$ e, na sequência, valores de $\mathrm{Nu}$ vs Ra. $\mathrm{O}$ Solver buoyantBoussinesqPimpleFoam conseguiu prever com coerência os dados experimentais de ambas as geometrias. Já os valores experimentais de velocidade são escassos e só havia para uma posição no cubo de (LIN; AKINS, 1983) preenchido com etileno glicol. Apesar de poucas opções foram visualizadas as curvas de velocidade em outros planos, evidenciando a simetria encontrada.

A caracterização da convecção natural na cavidade consistiu em visualizar uma série de resultados que explorassem a evolução dos padrões do campo de temperatura e velocidade. A variação de temperatura na condição de contorno para cada caso, foi feita de maneira gradual. Verificou-se que para baixos números de Rayleigh ocorria a manutenção dos padrões nas estruturas de fluidos aquecidas (em forma de cogumelos) que se formavam na base da cavidade. Sugerindo a desestabilização dessas estruturas para Rayleigh próximos à números de transição laminar-turbulento. 


\section{Conclusões}

Os picos de energia cinética demonstraram que estavam relacionados com o surgimento dos cogumelos ao longo da simulação e que ocorriam intermitentemente até que transferência de calor cessasse.

O surgimento dos cogumelos também ficaram demarcados quando observamos a variação de Nusselt nas paredes. A base da cavidade teve valores bem mais elevados de Nusselt, seguida das paredes laterais e valores mínimos na parede superior. Quando se observa separadamente os valores de Nusselt ao longo do tempo de simulação, verificase que o valor médio possui a maior contribuição dada pela influências das paredes laterais.

A grande vantagem do OpenFOAM é a liberdade com que o usuário tem em alterá-lo da maneira que lhe for conveniente. Nesse sentido, várias implementações foram feitas. Principalmente, para quando houve a necessidade da agitação/rotação da cavidade. Um novo Solver foi criado para impor a oscilação da cavidade e analisar a relação da variação angular com o tempo de aquecimento. Em outro caso, o Solver de convecção natural foi alterado para que pudesse ser trabalhado com o modelo de Simulação de Grandes Escalas. Este Solver serviu como ferramenta para a comprovação da correlação $\mathrm{Nu}$ vs Ra para a convecção natural turbulenta na cavidade paralelelípeda retangular.

As principais conclusões deste trabalho são:

- A utilização do OpenFOAM para simulação de convecção natural é um recurso que pode ser utilizado em diversas condições de contorno e geometria. Evidenciado nos resultados coerentes de $\mathrm{Nu}$ vs $\mathrm{Ra}$ e dados de velocidade.

- A caracterização da convecção natural da cavidade em que todas as faces foram aquecidas preencheu uma lacuna no estudo da evolução dos padrões do campo de temperatura.

- A agitação/rotação da cavidade influenciou acentuadamente no tempo de aquecimento.

- As implementações na criação de novos Solvers para problemas de agitação/rotação, para convecção natural com Simulação de Grandes Escalas e cálculos auxiliares, servirão como ferramenta para outros usuários do OpenFOAM.

Algumas sugestões para trabalhos futuros: 
- Análise da agitação/rotação mais detalhada da capacidade do equipamento que gerará a agitação e o tipo de alimento a ser agitado.

- Montagem experimental e simulações numéricas de modo a estudar melhor o mecanismo físicos da geração das estruturas aquecidas, assim como dos aspectos geométricos da cavidade e do processo de agitação. 


\section{Referências Bibliográficas}

ARPACI, V.; LARSEN, P. S. Convection Heat Transfer. New Jersey: Englewood cliffs: Prentice-Hall, 1984. 512 p.

AUGUSTO, P. E. D. Modelamento Matemático da Transferência de Calor Durante o Tratamento Térmico de Alimentos Líquidos Embalados. 220 p. Dissertação (Mestrado) - Universidade Estadual de Campinas. Faculdade de Engenharia de Alimentos (FEA), Campinas, SP, 2009.

AUGUStO, P. E. D. Efeito da Homogeneização à Alta Pressão (HAP) nas Propriedades Reológicas de Suco de Tomate. 191 p. Tese (Doutorado) Universidade Estadual de Campinas. Faculdade de Engenharia de Alimentos (FEA), Campinas, SP, 2012.

BEJAN, A. Convection Heat Transfer. 3a. ed.. ed. New York: John Wiley \& Sons, 2004. $673 \mathrm{p}$.

BEJAN, A.; KRAUS, A. Heat Transfer Handbook. Wiley, 2003. (Heat Transfer Handbook, v. 1). ISBN 9780471390152. Disponível em: <http://books.google. com.br/books?id=d4cgNG $\backslash$ IUq8C $>$.

CHHABRA, R.; RICHARDSON, J. Non-Newtonian Flow and Applied Rheology: Engineering Applications. Elsevier Science, 2011. (Engineering Applications Series). ISBN 9780080564937. Disponível em: <http://www.sciencedirect.com/ science/book/9780750685320>.

COURANT, R.; FRIEDRICHS, K.; LEWY, H. On the partial difference equations of mathematical physics. IBM J. Res. Dev., IBM Corp., Riverton, NJ, USA, v. 11, n. 2, p. 215-234, mar. 1967. ISSN 0018-8646. Disponível em: <http://dx.doi.org/10.1147/rd.112.0215>

DEITEL, H.; DEITEL, P. C ++ Como Programar. [S.l.]: Bookman, 2001. ISBN 9788573077407.

FAGHRI, A.; ZHANG, Y.; HOWELL, J. Advanced Heat and Mass Transfer. Global Digital Press, 2010. ISBN 9780984276004. Disponível em: $<$ https: //www.thermalfluidscentral.org/e-books/book-intro.php?b=37> 
FIGONI, P. I.; SHOEMAKER, C. F. Characterization of structure breakdown of foods from their flow properties. Journal of Texture Studies, Blackwell Publishing Ltd, v. 12, n. 3, p. 287-305, 1981. ISSN 1745-4603. Disponível em: <http://dx.doi.org/10.1111/j.1745-4603.1981.tb00540.x>

FUSEGI, T.; HYUN, J. M.; KUWAHARA, K. A numerical study of 3d natural convection in a cube: effects of the horizontal thermal boundary conditions. Fluid Dynamics Research, v. 8, n. 5-6, p. 221-230, 1991. Disponível em: <http://iopscience.iop.org/1873-7005/8/5-6/A04>.

FUSEGI, T. et al. A numerical study of three-dimensional natural convection in a differentially heated cubical enclosure. Int. J. Heat and Mass Transfer, v. 34, n. 6, p. 1543?1557, 1991. Disponível em: <http://dx.doi.org/10.1016/ 0017-9310(91)90295-P>

GUMERATO, H. F. Estudo da Transferência de Calor Transiente por Agitação Interminente em Embalagens. 118 p. Tese (Doutorado) — Universidade Estadual Paulista (UNESP), Botucatu, SP, 2004.

HERSCHEL, W.; BULKLEY, R. Konsistenzmessungen von gummi-benzollösungen. Kolloid-Zeitschrift, Springer-Verlag, v. 39, n. 4, p. 291-300, 1926. ISSN 0368-6590. Disponível em: <http://dx.doi.org/10.1007/BF01432034>

HIDDINK, J. Natural convection heating of liquids, with reference to sterilization of canned food. 128 p. Tese (Doutorado) - Wageningen University UR, Wagenigen, Netherlands, 1975. Disponível em: <http://http://library.wur.nl/WebQuery/ clc/104144>

HSIEH, S. S.; WAND, C. Y. Experimental-study of 3-dimensional natural convection in enclosures with different working fluids. Int. J. Heat Mass Transfer, v. 37, n. 17, p. 2687-2698, 1994.

HSIEH, S. S.; YANG, S. S. Transient three-dimensional natural convection in a rectangular enclosure. Int. J. Heat Mass Transfer, v. 39, n. 1, p. 13-26, 1996. Disponível em: <http://dx.doi.org/10.1016/S0017-9310(96)85002-2>.

JASAK, H. Error Analysis and Estimation for the Finite Volume Method with Applications to Fluid Flows. 394 p. Tese (Doutorado) - Imperial College, University of London, London, 1996.

KARYPIS, G.; KUMAR, V. Multilevel algorithms for multi-constraint graph partitioning. In: Proceedings of the 1998 ACM/IEEE Conference on Supercomputing. Washington, DC, USA: IEEE Computer Society, 1998. (SC '98), p. 1-13. ISBN 0-89791-984-X. Disponível em: <http://glaros.dtc.umn.edu/ gkhome/node/90>

- Multilevel k-way partitioning scheme for irregular graphs. J. Parallel

Distrib. Comput., Academic Press, Inc., Orlando, FL, USA, v. 48,

n. 1, p. 96-129, jan. 1998. ISSN 0743-7315. Disponível em: <http: //dx.doi.org/10.1006/jpdc.1997.1404>. 
KOLMOGOROV, A. N. The local structure of turbulence in incompressible viscous fluid for very large reynolds numbers. Proceedings: Mathematical and Physical Sciences, The Royal Society, v. 434, n. 1890, p. 9-13, 1991. ISSN 09628444. Disponível em: <http://www.jstor.org/stable/51980>

LEONARD, A. Energy cascade in large-eddy simulations of turbulent fluid flows. In: FRENKIEL, F.; MUNN, R. (Ed.). Turbulent Diffusion in Environmental PollutionProceedings of a Symposium held at Charlottesville. Elsevier, 1975, (Advances in Geophysics, v. 18, Part A). p. 237 - 248. Disponível em: <http://www.sciencedirect.com/science/article/pii/S0065268708604641>.

LIN, Y. S. An experimental study of flow patterns and heat transfer by natural convection inside cubical enclosures. 102 p. Dissertação (Mestrado) - Kansas State University, Manhattan, Kansas, 1982.

LIN, Y. S.; AKINS, R. G. An experimental study of flow patterns and heat transfer by natural convection inside cubical enclosures. ASME HTD, v. 26, p. 35-42, 1983.

MALISKA, C. R. Transferência de Calor e Mecânica dos Fluidos Computacional. Rio de Janeiro, Brasil: Ed. LTC, 2004. 433 p.

MARIĆ, T.; HÖPKEN, J.; MOONEY, K. The OpenFOAM Technology Primer. [S.l.]: Sourceflux UG, 2014. ISBN 978-3-00-046757-8.

OPENCFD. OpenFOAM 2.3 - The Open Source CFD Toolbox - Programmer's Guide. [S.l.], 2014. Disponível em: <http://foam.sourceforge.net/docs/Guides-a4/ ProgrammersGuide.pdf $>$.

. OpenFOAM 2.3 - The Open Source CFD Toolbox - User Guide. [S.l.], 2014. Disponível em: <http://foam.sourceforge.net/docs/Guides-a4/UserGuide.pdf>

PATANKAR, S. Numerical Heat Transfer and Fluid Flow. [S.1.]: Taylor \& Francis, 1980. (Series in computational methods in mechanics and thermal sciences). ISBN 9780891165224.

PERIć, M.; KESSLER, R.; SCHEUERER, G. Comparison of finite-volume numerical methods with staggered and colocated grids. Computers $\&$ Fluids, v. 16, n. 4, p. 389 - 403, 1988. ISSN 0045-7930. Disponível em: <http://www.sciencedirect.com/science/article/pii/0045793088900242>.

POPE, S. B. Turbulent flows. Cambridge: Cambridge University Press, 2000. Réimpressions (avec des corrections) : 2001, 2003, 2005, 2008, 2010. ISBN 0-521-59125-2. Disponível em: <http://opac.inria.fr/record=b1096703>.

RHIE, C. M.; CHOW, W. L. Numerical study of the turbulent flow past an airfoil with trailing edge separation. AIAA Journal, v. 21, n. 11, p. 1525 - 1532, 1983. Disponível em: <http://arc.aiaa.org/doi/abs/10.2514/3.8284>. 
RUSCHE, H. Computational Fluid Dynamics of Dispersed Two-Phase Flows at High Phase Fractions. 343 p. Tese (Doutorado) - Imperial College, University of London, London, 2003.

SAAD, Y. Iterative Methods for Sparse Linear Systems. 2nd. ed. Philadelphia, PA, USA: Society for Industrial and Applied Mathematics, 2003. ISBN 0898715342.

SILVA, L. F. L. R. Desenvolvimento de Metodologia para Simulação de Escoamentos Polidispersos Usando Código Livre. 262 p. Tese (Doutorado) - Universidade Federal do Rio de Janeiro (COPPE), Rio de Janeiro, RJ, 2008.

SMAGORINSKY, J. General circulation experiments with the primitive equations. i. the basic experiment. Monthly Weather Review, v. 91, n. 3, p. 99-164, 1963. Disponível em: <http://docs.lib.noaa.gov/rescue/mwr/091/mwr-091-03-0099. pdf $>$.

SPARROW, E. M.; HUSAR, R. B.; GOLDSTEIN, R. J. Observations and other characteristics of thermals. Journal of Fluid Mechanics, v. 41, p. 793-800, 1970.

STOLL, R. LES of Turbulent Flows: Lecture 3(ME EN 7960-003). [S.1.]: Department of Mechanical Engineering University of Utah, 2014.

TOLlini, P. M. Convecção Transiente em uma Cavidade 3D: Uma Aplicação ao Aquecimento de Produtos Embalados. 125 p. Dissertação (Mestrado) Universidade de Brasília, Brasília, DF, 1996.

TORO, E. Riemann Solvers and Numerical Methods for Fluid Dynamics: A Practical Introduction. Springer, 2009. ISBN 9783540498346. Disponível em: <http://link.springer.com/book/10.1007\%2Fb79761>.

VERSTEEG, H. K.; MALALASEKERA, W. An Introduction to Computational Fluid Dynamics: The Finite Volume Method. Reino Unido: Longman Scientific \& Technical, 1995. ISBN 0582218845.

VILLIERS, E. de. The Potential of Large Eddy Simulation for the Modeling of Wall Bounded Flows. 375 p. Tese (Doutorado) - Department of Mechanical Engineering, Imperial College of Science, Technology and Medicine, University of London, London, 2006.

WELLER, H. G. Derivation, modelling and solution of the conditionally averaged two-phase flow equations. East Lansing, Michigan, 2002.

WELLER, H. G. et al. A tensorial approach to computational continuum mechanics using object-oriented techniques. Comput. Phys., American Institute of Physics Inc., Woodbury, NY, USA, v. 12, n. 6, p. 620-631, nov. 1998. ISSN 0894-1866. Disponível em: <http://dx.doi.org/10.1063/1.168744>

YANG, D. $C++$ and Object-Oriented Numeric Computing for Scientists and Engineers. [S.l.]: Springer New York, 2001. ISBN 9780387989907. 
A. Implementações Numéricas

\section{Apêndice A}

\section{Implementações Numéricas}

\section{A.1 Implementação Numérica do Solver para o Problema de Agitação da Cavidade}

Código A.1: Arquivo principal g_buoyantBoussinesqPimpleFoam.C

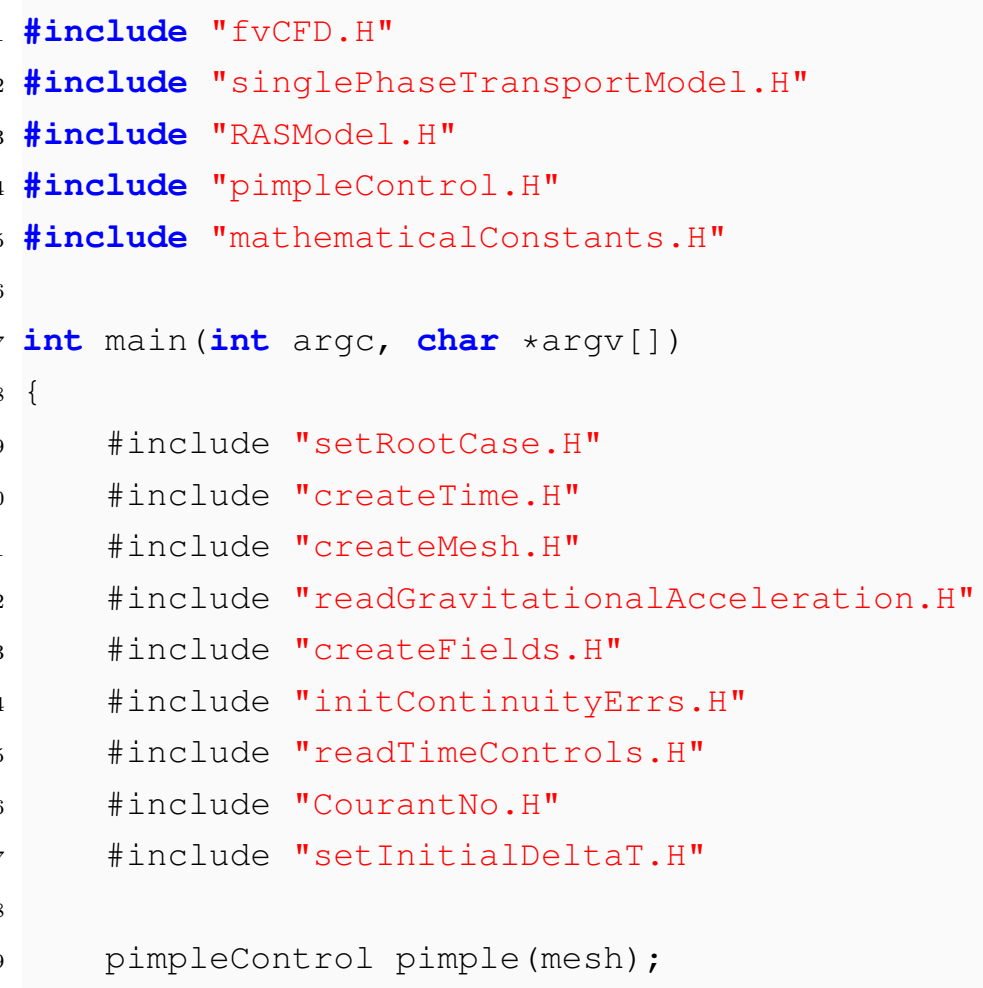




\section{A. Implementações Numéricas}

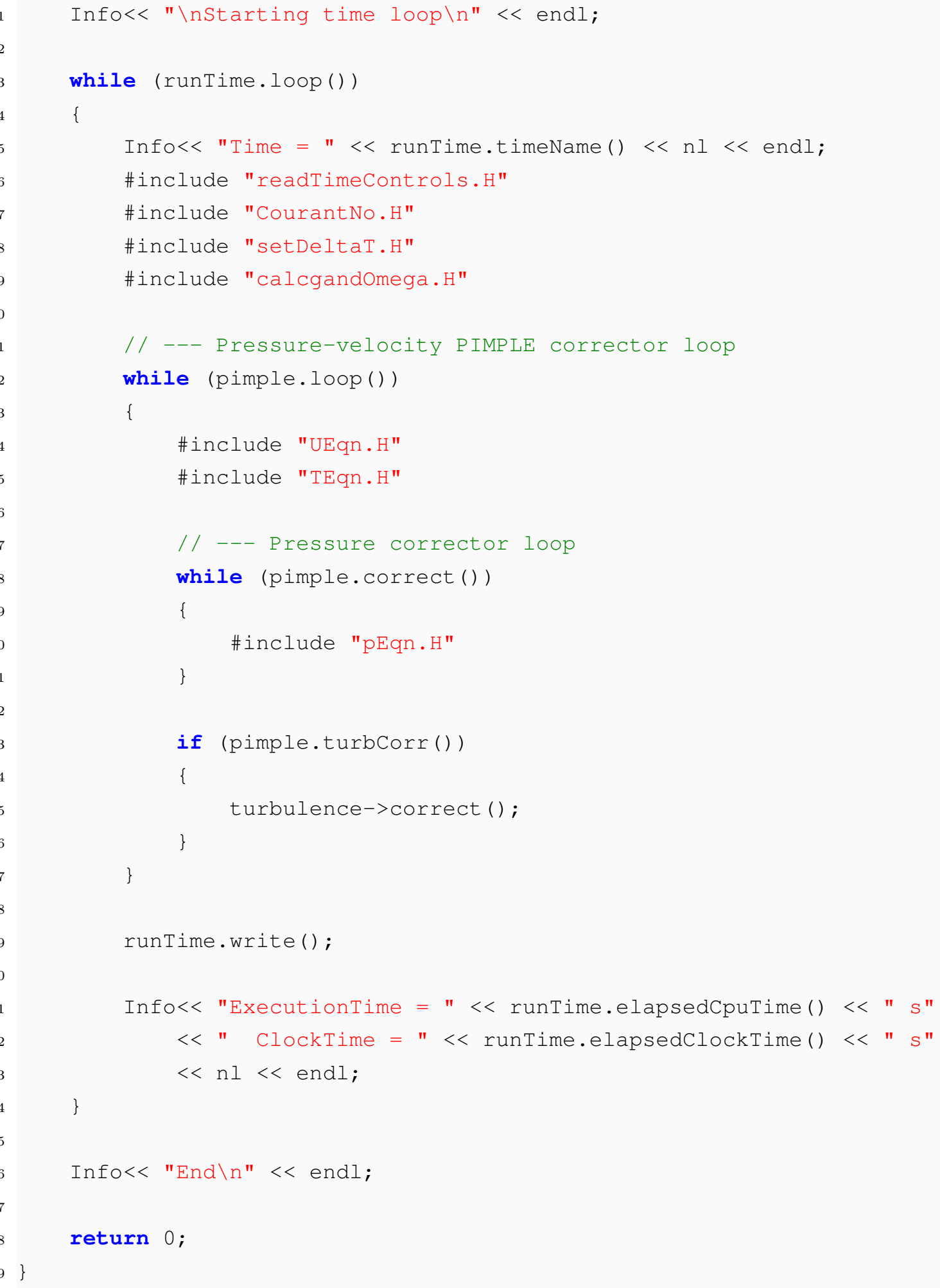

Código A.2: Arquivo de Criação de Campos createFields.H

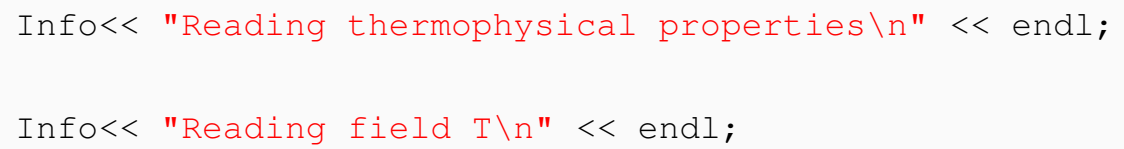




\section{A. Implementações Numéricas}

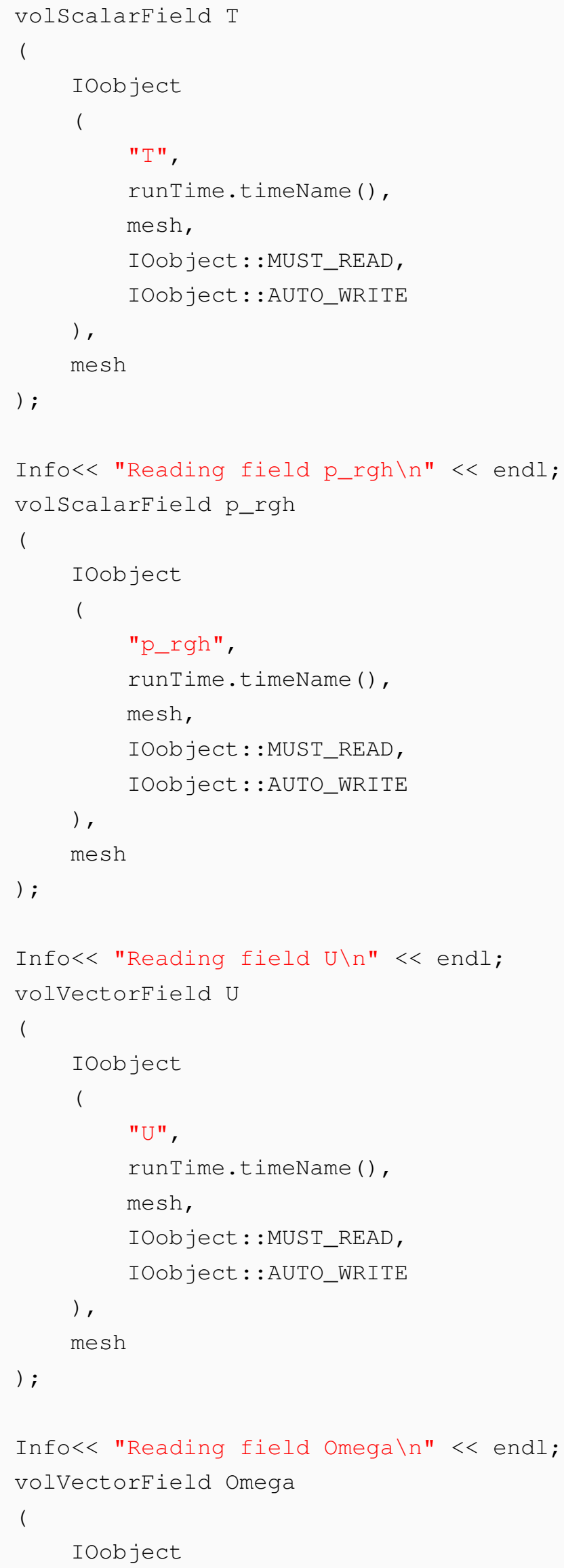




\section{A. Implementações Numéricas}

49

50

51

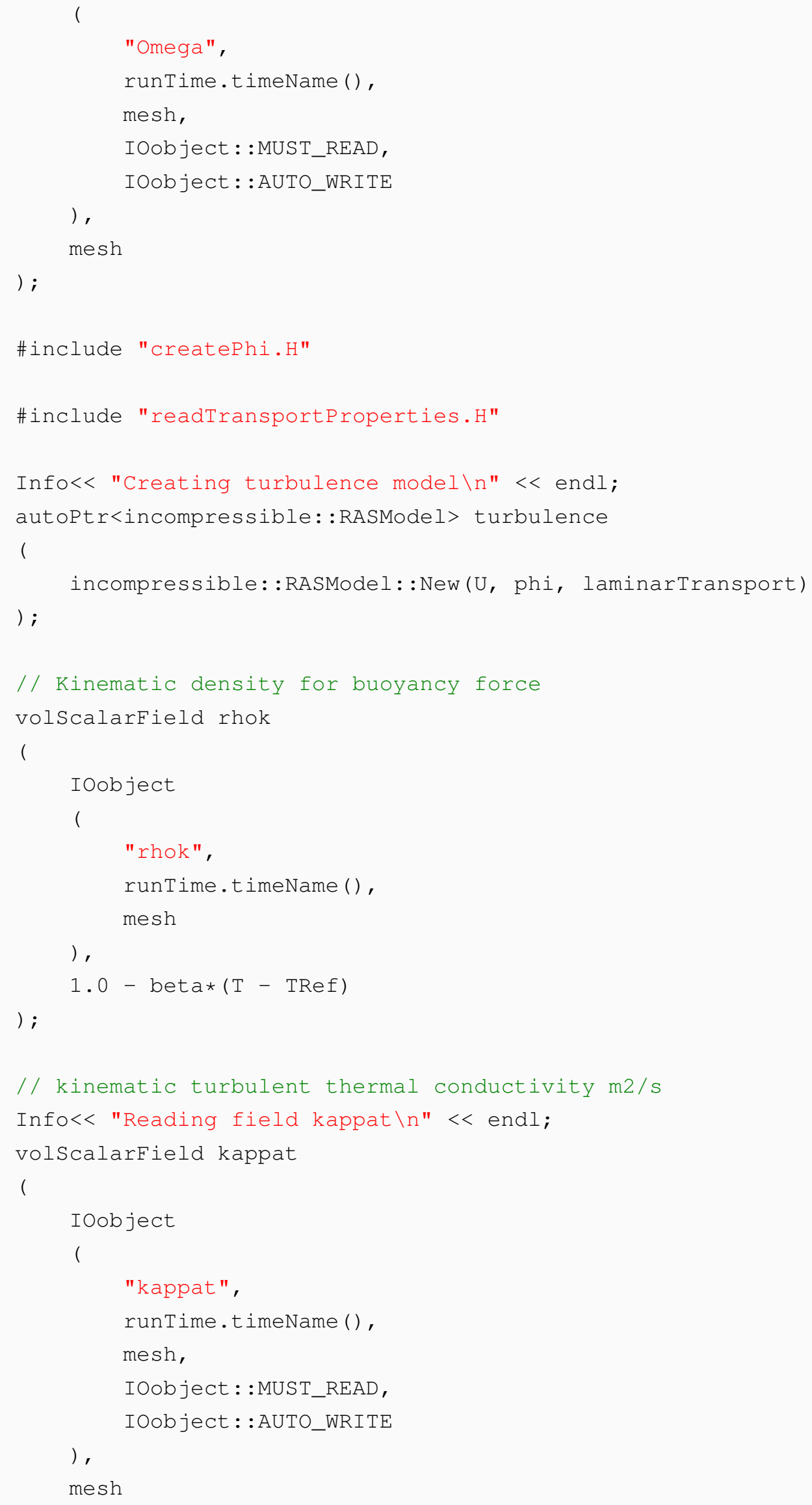




\section{A. Implementações Numéricas}

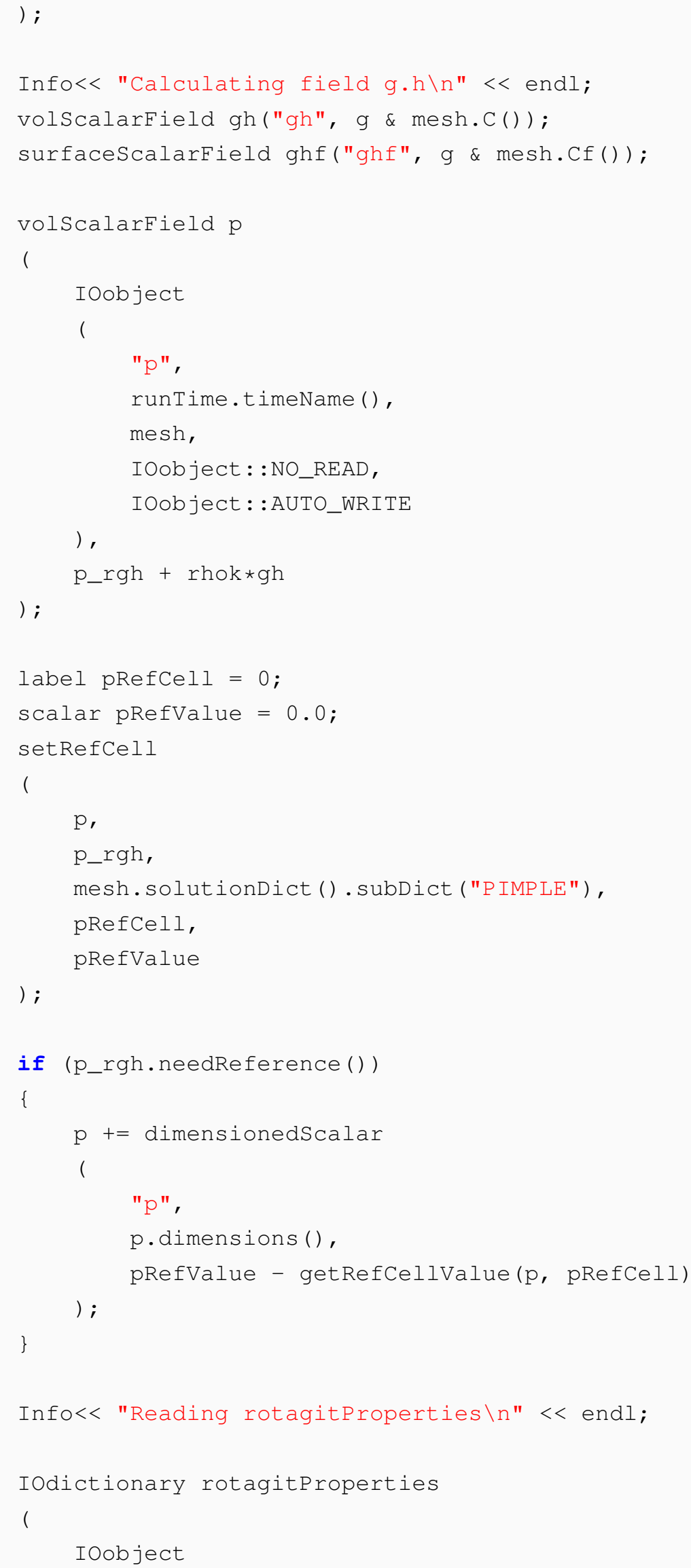




\section{A. Implementações Numéricas}

139

140

141

142

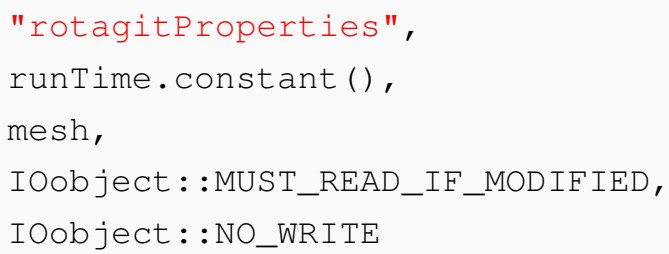




\section{A. Implementações Numéricas}

Código A.3: Arquivo calcgandomega.H

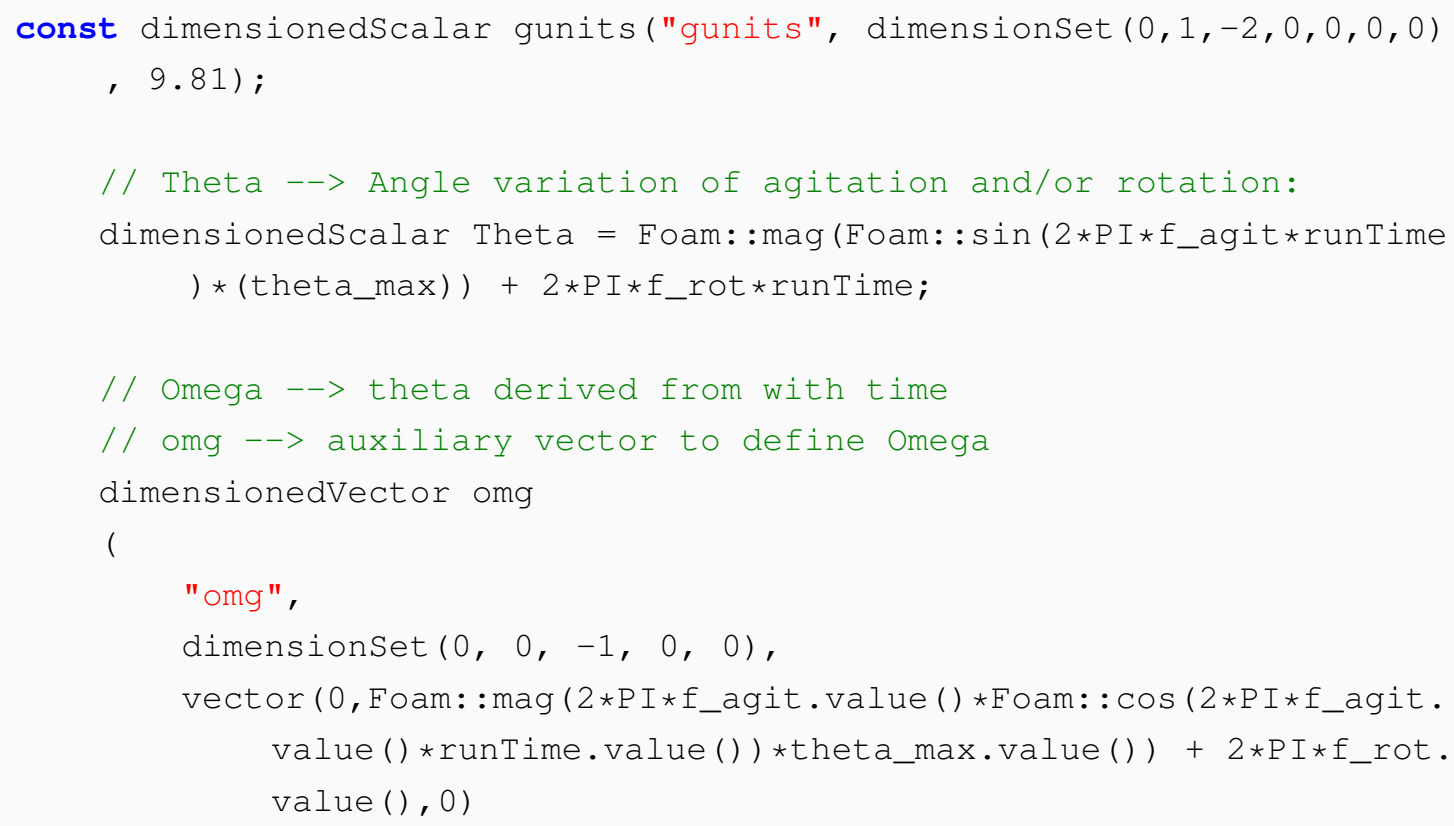




\section{A. Implementações Numéricas}

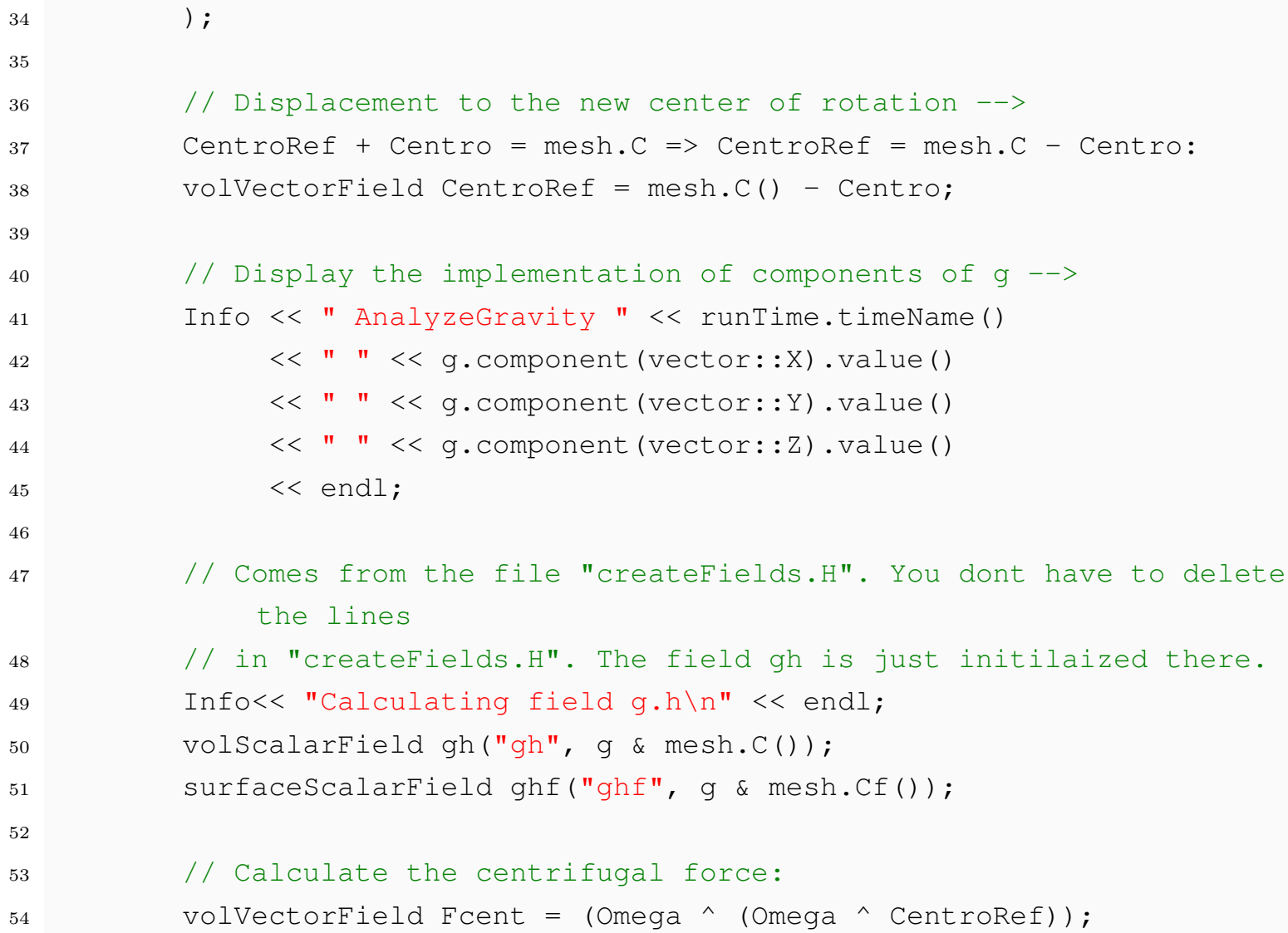

// Displacement to the new center of rotation --> CentroRef + Centro $=$ mesh. $\mathrm{C}=>$ CentroRef $=$ mesh.C - Centro: volvectorfield CentroRef = mesh.C() - Centro;

\section{Código A.4: Arquivo UEqn.H}

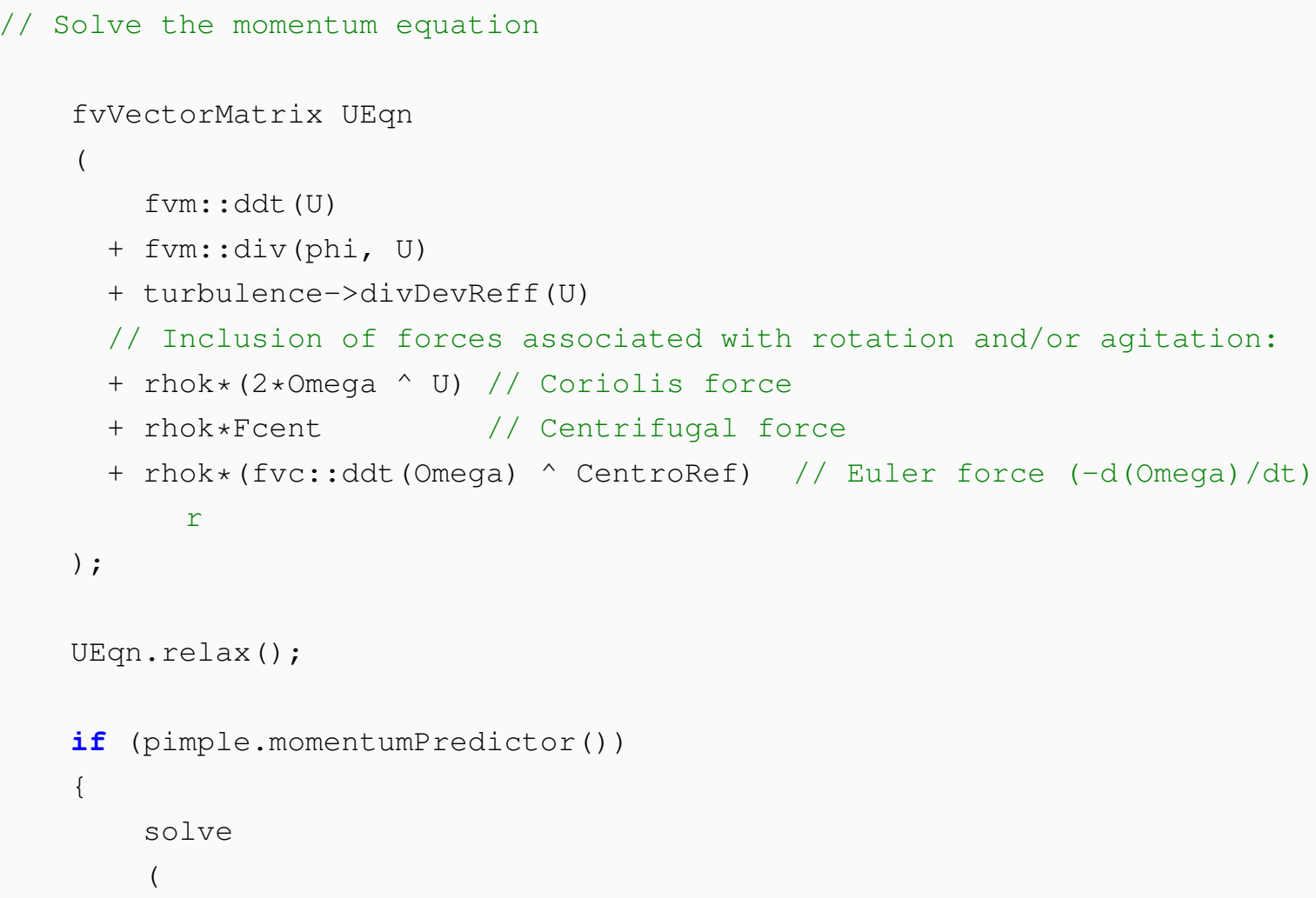




\section{A. Implementações Numéricas}

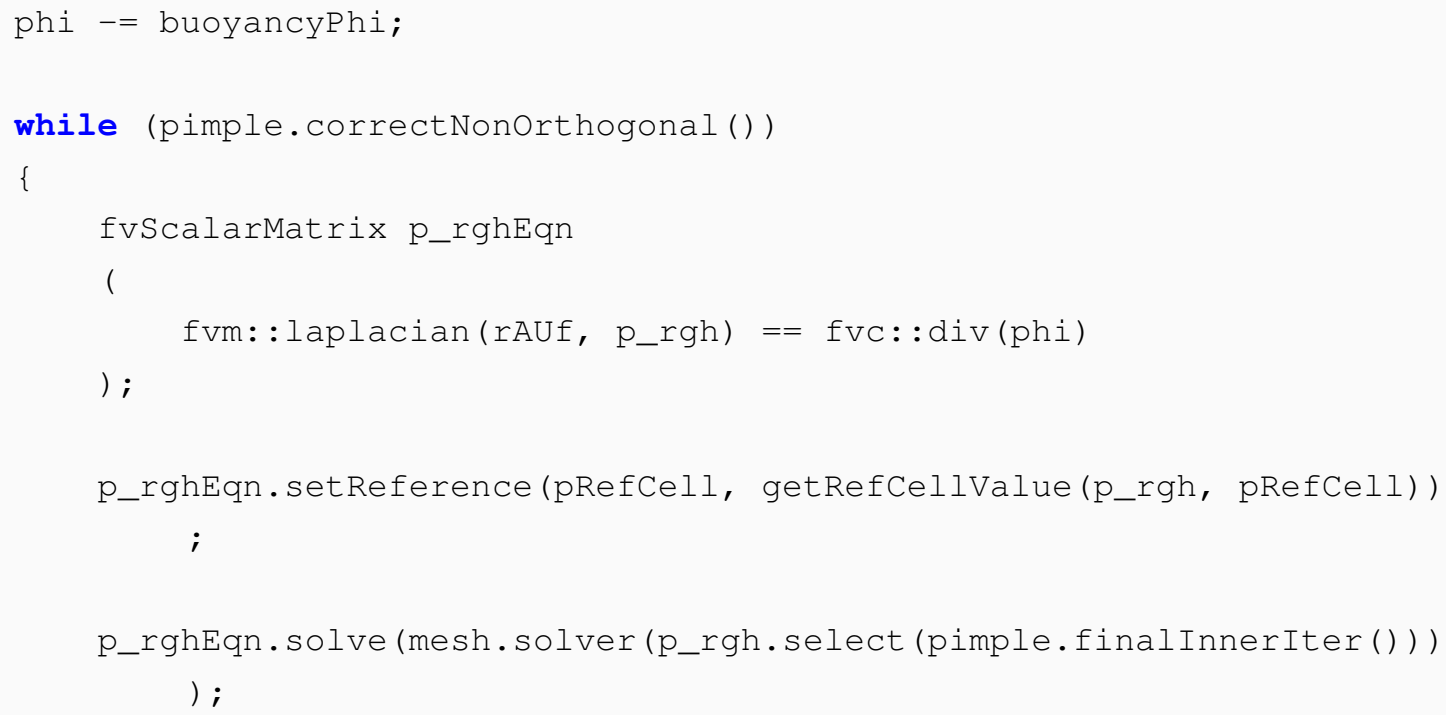


A. Implementações Numéricas

52

$53\}$ 